\title{
Investigating the mechanism by which the atypical antipsychotic clozapine reduces disease in experimental autoimmune encephalomyelitis
}

\author{
By \\ Pirooz Zareie
}

A thesis submitted to the Victoria University of Wellington in fulfilment of the requirements for the degree of Doctor of Philosophy Victoria University of Wellington 


\begin{abstract}
Multiple sclerosis (MS) is an inflammatory disease of the central nervous system characterized by damage to the myelin sheaths that surround nerve axons. Inflammatory damage to the myelin sheath leads to severe physical disability in patients. Whereas approved disease modifying treatments are available for relapsing-remitting forms of MS, there are no approved treatments for the progressive stages, leaving approximately 50\% of MS sufferers without treatment. Therefore, there is an urgent need for development of effective alternatives.
\end{abstract}

Atypical antipsychotic agents used for treating schizophrenia have recently been recognized for their immune-modifying properties and our laboratory has shown previously that treating mice with risperidone or clozapine reduces the severity of disease in experimental autoimmune encephalomyelitis (EAE), an animal model for MS. Although atypical antipsychotic agents like clozapine have been used in the clinic for almost 60 years, there is very little experimental data that describes the mechanism by which atypical antipsychotic agents like clozapine are able to modify the immune response. This thesis aimed to describe the immunological mechanisms by which clozapine is able to reduce EAE disease and to determine the underlying cellular signalling alterations that occur during treatment to facilitate immune modifying properties.

In vitro experiments showed that clozapine can impair induction of Th1 and Th17 cells while promoting the differentiation of iTreg and increasing Foxp3 expression. However, although clozapine effectively delayed disease onset and reduced the severity of EAE, the therapeutic effect of clozapine was not associated with impaired capacity to induce antigen specific Th1 or Th17 responses in the periphery. Moreover, Treg function was dispensable for disease protection by clozapine. Instead, disease protection by clozapine was associated with a suppressed state of activation in CNS resident microglia and infiltrating monocytes assessed by flow cytometric measurement of activation associated receptor expression. In vitro experiments using primary macrophage cell culture revealed that clozapine can alter the activation of activated macrophages towards a less inflammatory state directly. Interestingly, the altered state of activation in primary macrophages was not associated with detectable changes in cell signalling pathways known to mediate activation.

This thesis demonstrated that clozapine treatment protects from EAE by a multi-faceted immunological mechanism that likely involves modifying multiple pathways and cell types during EAE and may be of therapeutic benefit to MS patients in the progressive stages of 
disease. Finally, this thesis also has relevance to psychiatry as it demonstrates that clozapine has potential to alter cellular immune responses. 


\section{Acknowledgements}

Firstly, I would like to thank my supervisor Professor Anne La Flamme for her continued encouragement, guidance and support in the last four years. This work was made possible by her assistance and constructive criticism when developing experimental approaches.

I would also like to thank my secondary supervisor Dr. Melanie McConnell for her support and guidance throughout the last three years.

I am very grateful to the entire LAF lab group for providing a friendly work environment. In particular, I would like to thank Laura Green, Sarrabeth Stone and Madeleine White for all of the teaching and providing assistance from the beginning. This work could not have been done without your help. I would like to thank Nikki Templeton, Vimal Patel and Katharina Robichon for providing help in the lab during the long experiments. I would also like to thank the entire AM group for providing a supportive and friendly office and lab environment.

I would like to thank Dr. Janet Pitman for her guidance in some of my experiments, she has been extremely helpful and generous with her knowledge.

Thank you to the Neurological Foundation for funding the research project. Thank you to the Australasian Society for Immunology, Wellington Medical Research Foundation, Centre for Biodiscovery and the Faculty of Science for financial support to present this work at international conferences.

Finally, I would like to thank my friends and family for your understanding, support and encouragement in the last four years. 


\section{Table of Contents}

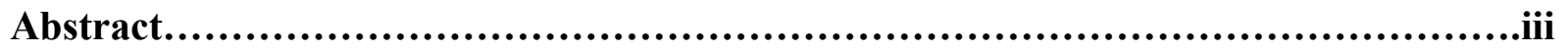

Acknowledgements ......................................................................................................................... v

Table of Contents ........................................................................................................................... vi

List of

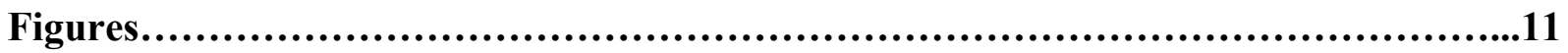

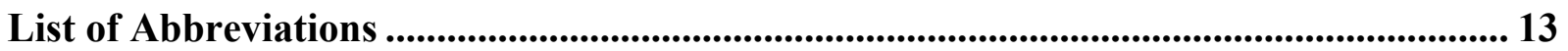

Chapter 1: Introduction.......................................................................................... 16

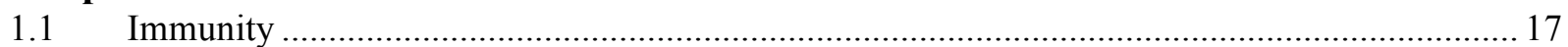

1.2 The innate immune response .................................................................................... 17

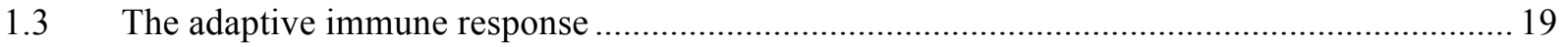

1.3.1 Antigen recognition by $T$ cells......................................................................... 20

1.3.2 Antigen recognition by $B$ cells ......................................................................... 21

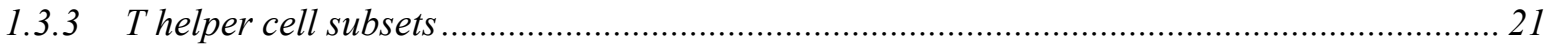

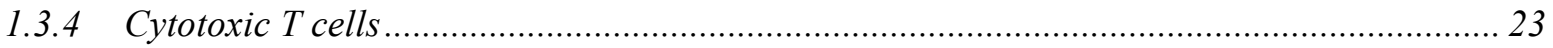

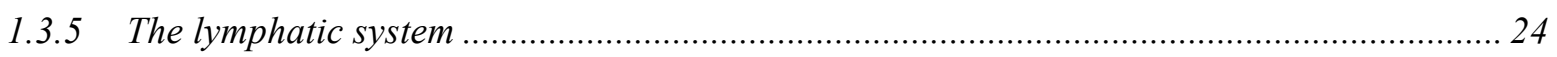

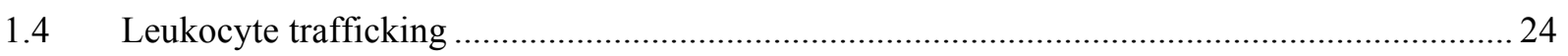

1.5 Antigen presentation - bridging the innate and adaptive immune response ...............................2

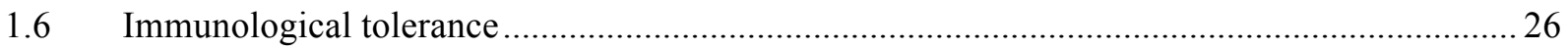

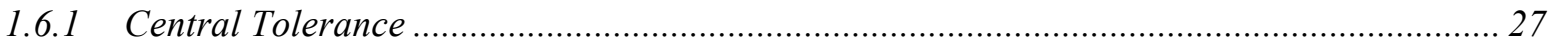

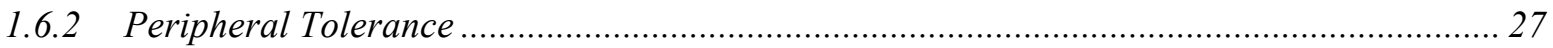

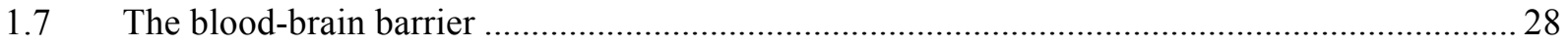

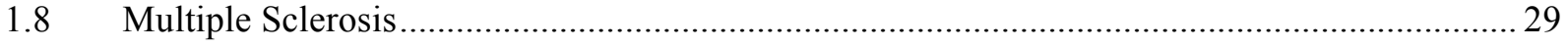

1.9 Experimental autoimmune encephalomyelitis as a model for CNS inflammation .....................31

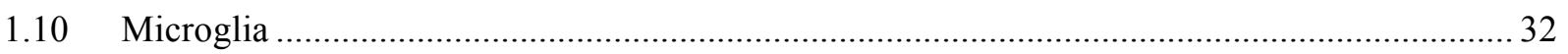

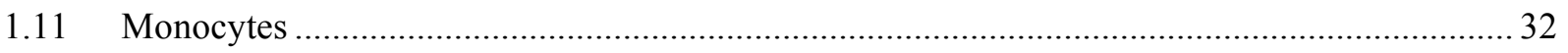

1.12 Atypical antipsychotic agents and inflammation .............................................................. 33

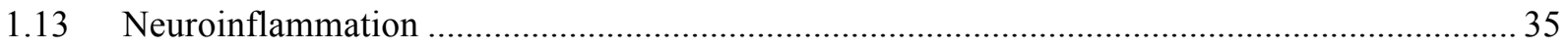

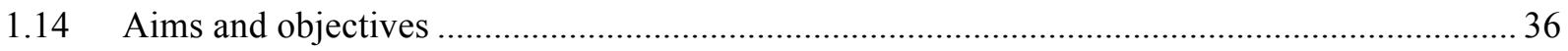

Chapter 2: Materials and Methods.................................................................................... 37

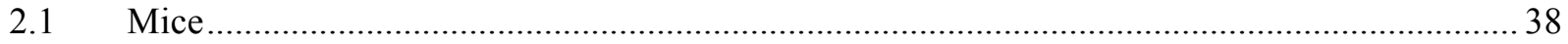

2.2 Induction of experimental autoimmune encephalomyelitis .............................................. 38

2.2.1 Induction of active EAE ………………………………………………………… 38

2.2.2 Induction of adoptively transferred EAE …………………………………………..... 39

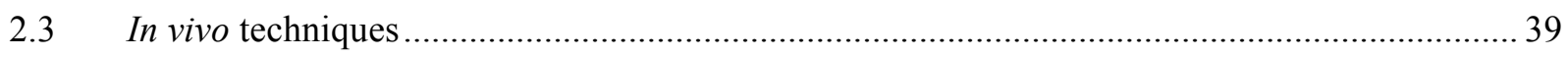




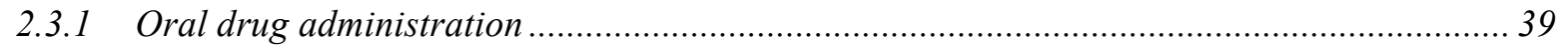

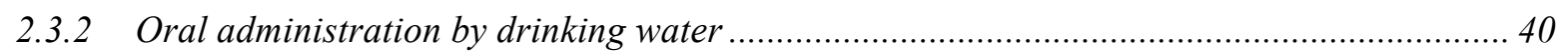

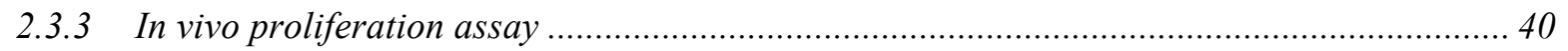

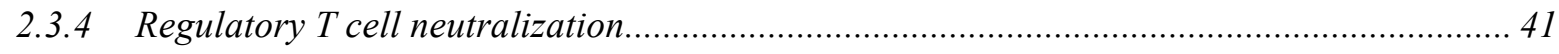

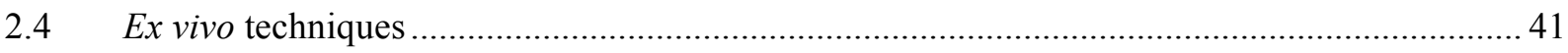

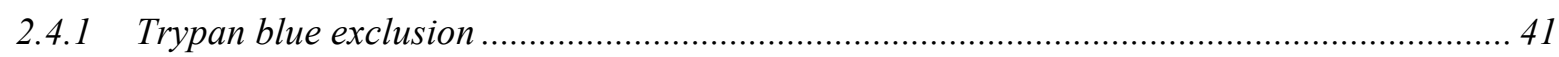

2.4.2 Processing and isolation of spleen into single cell suspensions .......................................... 41

2.4.3 Processing and isolation of lymph nodes into single cell suspensions .................................. 42

2.4.4 Processing and isolation of brain into single cell suspension ............................................. 42

2.4.5 Processing and isolation of spinal cord into single cell suspension ..................................... 43

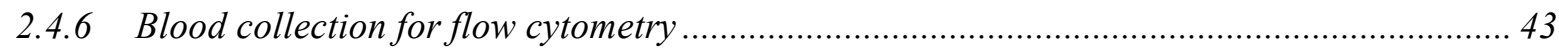

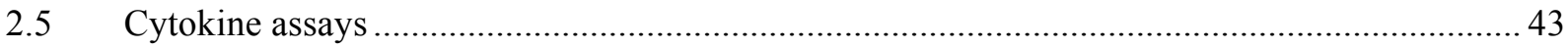

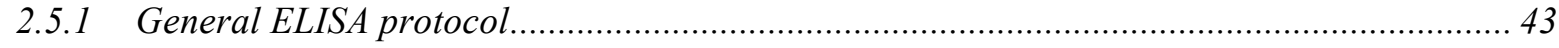

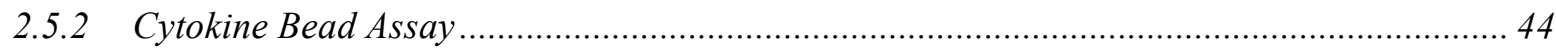

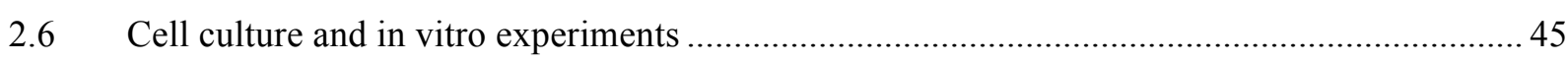

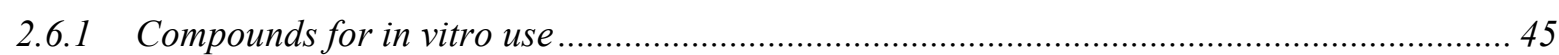

2.6.2 Culture and maintenance of $R A W 264.7$ macrophage cell line …........................................ 45

2.6.3 Isolation and culture of bone marrow derived macrophages .............................................. 46

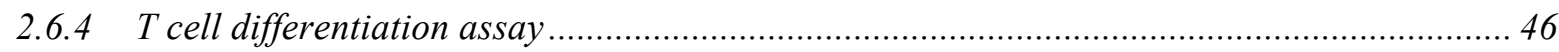

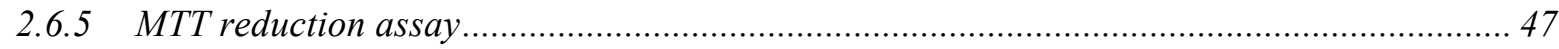

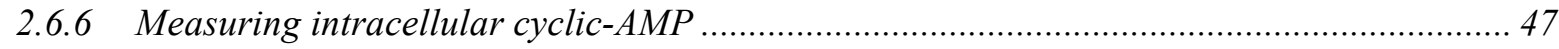

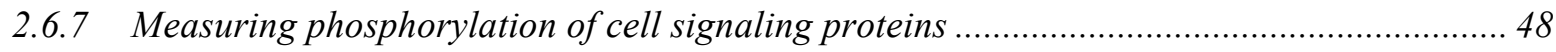

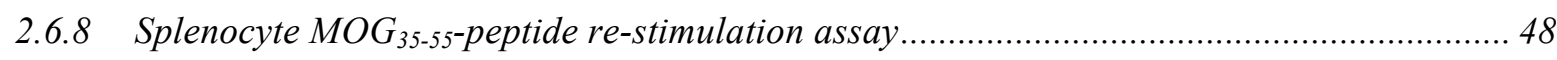

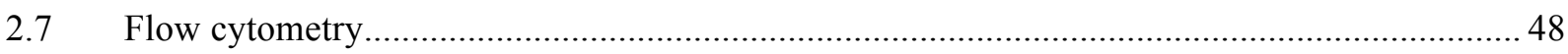

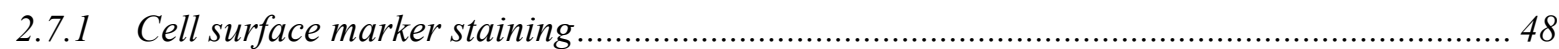

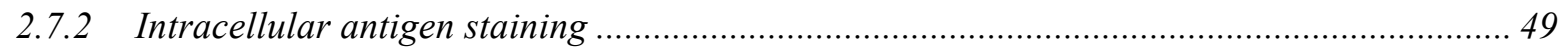

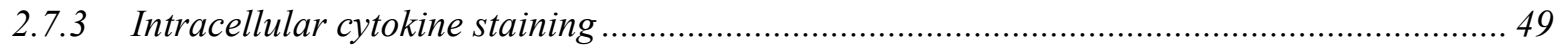

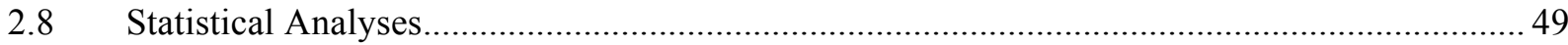

Chapter 3: Using clozapine to treat experimental autoimmune encephalomyelitis...... 51

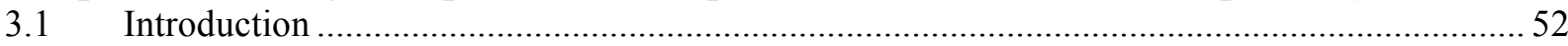

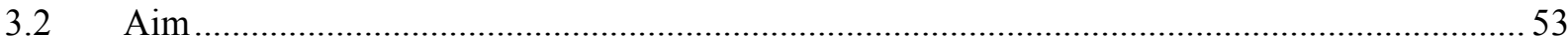

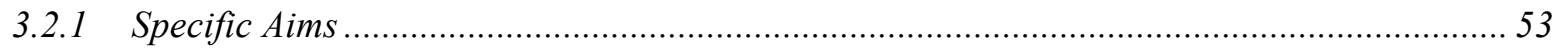

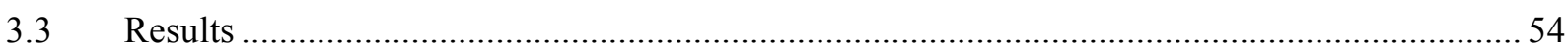

3.3.1 Clozapine treatment attenuates the disease course of EAE .................................................. 54

3.3.2 Clozapine administered after peak disease is effective at reducing disease in EAE ............ 60

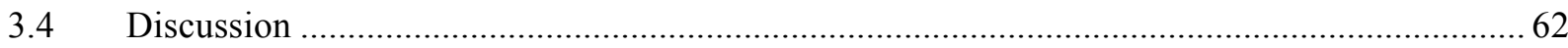

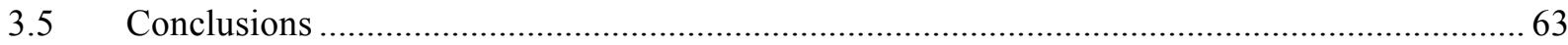




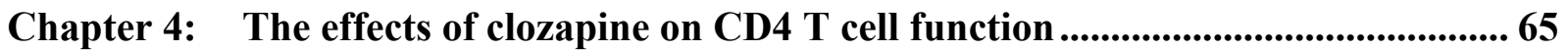

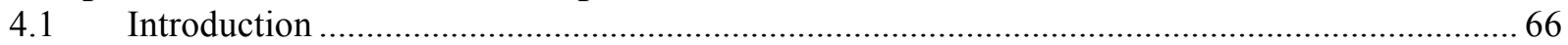

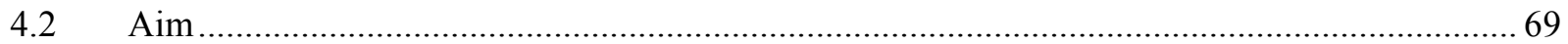

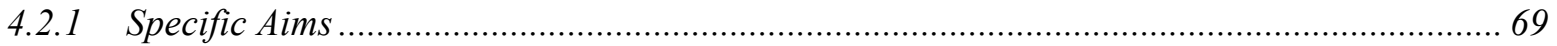

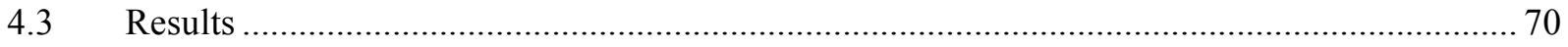

4.3.1 Clozapine reduces the viability of $\mathrm{CD}^{+} \mathrm{T}$ cells in a concentration dependent manner........ 70

4.3.2 Clozapine did not alter Th1 and Th17 differentiation but promoted Treg expansion ........... 72

4.3.3 $\quad \beta$-catenin expression was unaltered by clozapine in iTreg .................................................. 77

4.3.4 Treatment with clozapine did not alter peripheral Th cell subsets during EAE ................... 79

4.3.5 Clozapine did not alter $M O G_{35-55}$-specific $T$ cell expansion after EAE induction .................. 83

4.3.6 The clozapine-mediated delay and reduction in EAE was not mediated by Treg..................85

4.3.7 Clozapine did not alter the encephalitogenic capacity of myelin-specific T cells .................89

4.3.8 Clozapine did not protect from adoptively transferred EAE disease ................................... 91

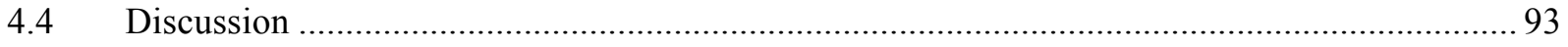

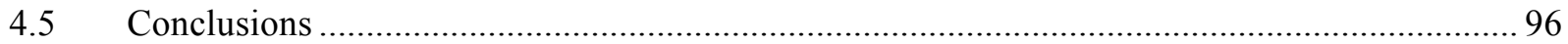

Chapter 5: The effect of clozapine on the activation of myeloid cells............................ 98

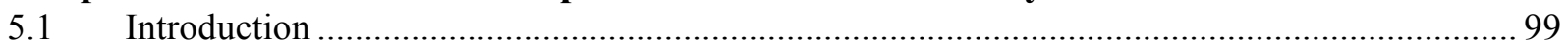

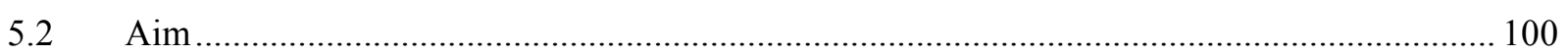

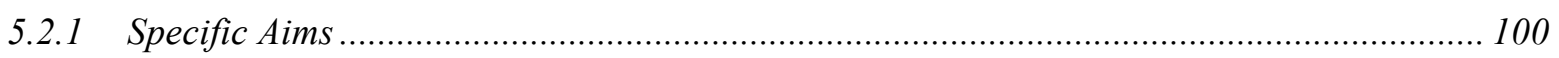

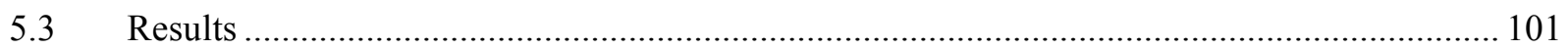

5.3.1 Clozapine limits peripheral infiltration of leukocytes into the CNS 15 d.p.i ...................... 101

5.4 Clozapine treated mice express lower levels of inflammatory markers on microglia and CNS

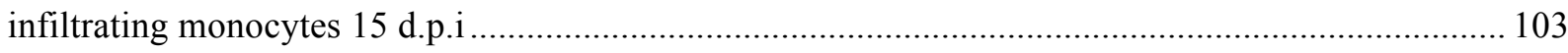

5.5 CNS infiltration of myeloid cells in clozapine treated mice parallels infiltration in vehicle treated mice.

5.5.1 Clozapine inhibits the activation of microglia and infiltrating monocytes in the spinal cord

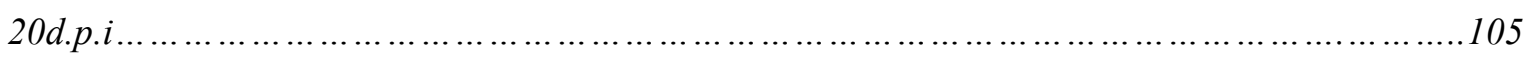

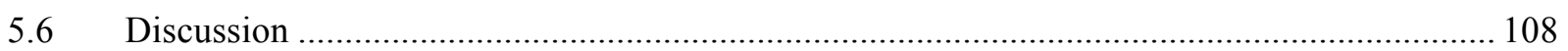

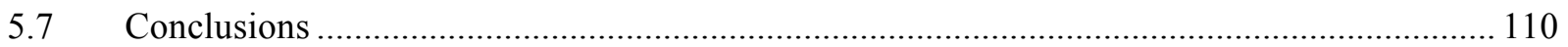

Chapter 6: The effect of clozapine on macrophage activation ......................................... 112

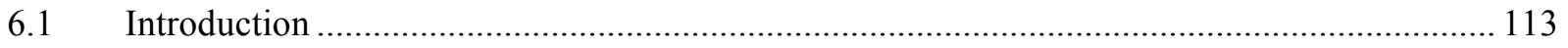

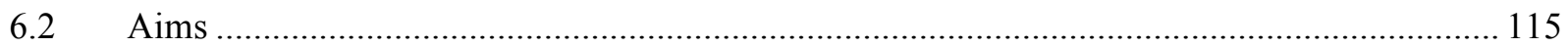

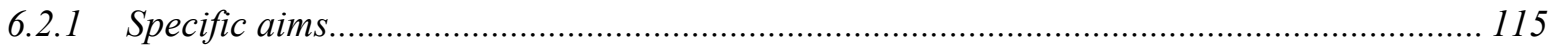

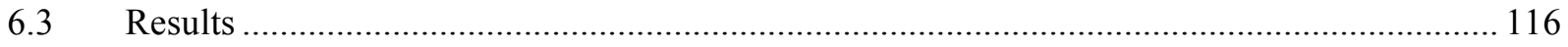

6.3.1 Macrophages expressed $D 1 R, D 2 R$ and $\beta$-adrenergic receptors ................................. 116

6.4 Dopamine inhibited IL-12 and increased IL-10 secretion in LPS stimulated macrophages.... 117 
6.5 Clozapine suppressed IL-12 and increased IL-10 production in lipopolysaccharide stimulated primary macrophages

6.5.1 Risperidone suppressed IL-12 and increased IL-10 production in macrophages ............... 121

6.5.2 Forskolin inhibited secretion of IL-12 in macrophages .................................................. 122

6.5.3 Clozapine did not alter cAMP production in lipopolysaccharide-stimulated primary

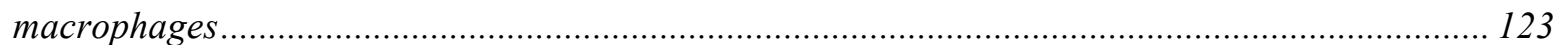

6.5.4 The Akt-GSK3 pathway modulated LPS-induced cytokine production................................ 123

6.5.5 Clozapine did not alter LPS-induced phosphorylation of signaling pathways.................... 129

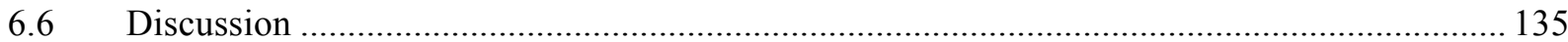

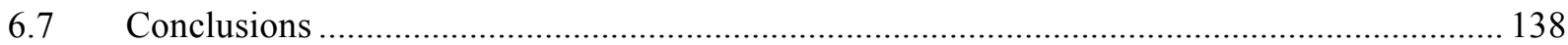

Chapter 7: Investigation into novel therapeutic strategies for multiple sclerosis ....... 140

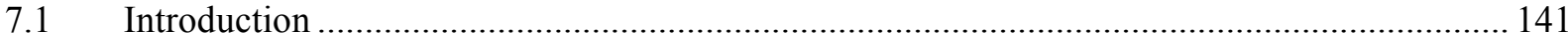

7.1.1 L-DOPA and clozapine as potential therapeutics for multiple sclerosis ........................... 141

7.1.2 Risperidone and fluoxetine as potential treatments for multiple sclerosis ........................ 142

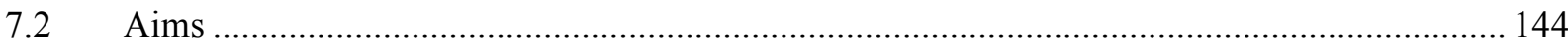

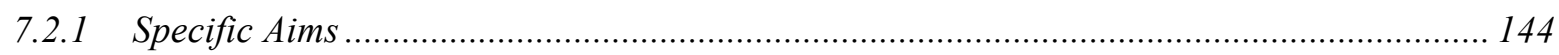

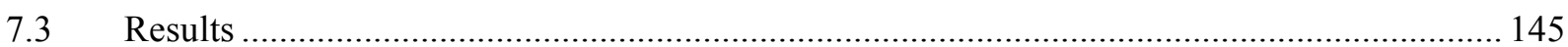

7.3.1 Levodopa/Carbidopa treatment does not alter the disease course of EAE ........................ 145

7.3.2 L-DOPA does not enhance clozapine protection during EAE ......................................... 147

7.3.3 Risperidone and fluoxetine do not synergize to enhance protection from EAE.................. 150

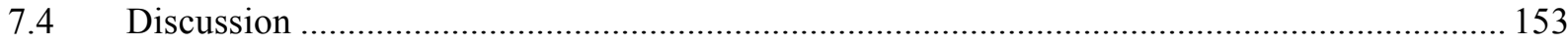

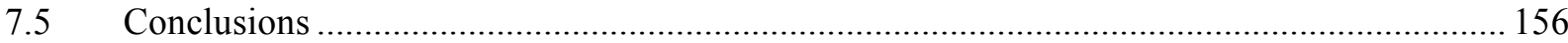

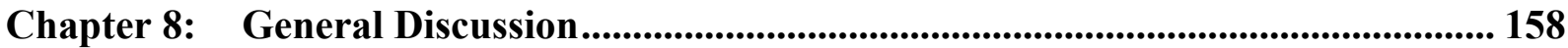

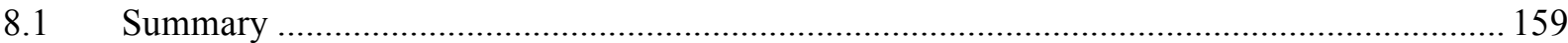

8.2 The potential mechanism of action of clozapine during EAE ................................................ 159

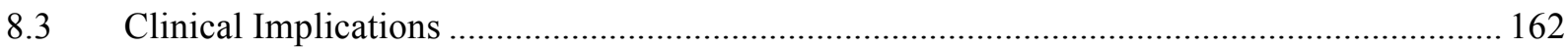

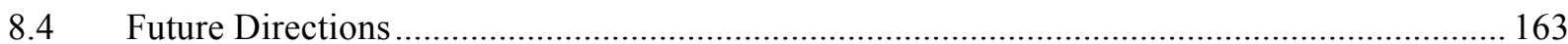

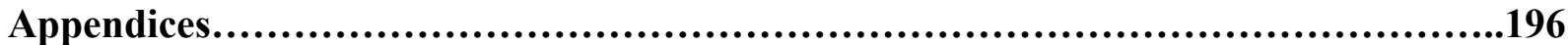

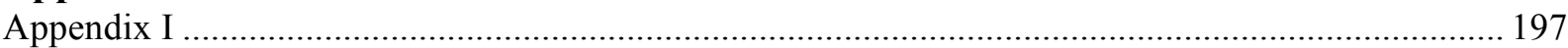

Enhanced disease reduction using clozapine, an atypical antipsychotic agent, and glatiramer acetate combination therapy in experimental autoimmune encephalomyelitis .............................................. 197

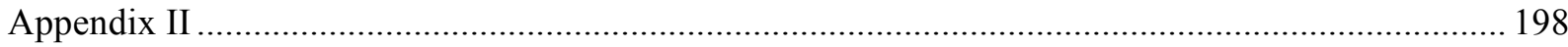

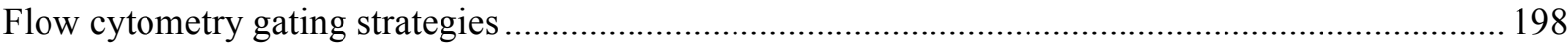

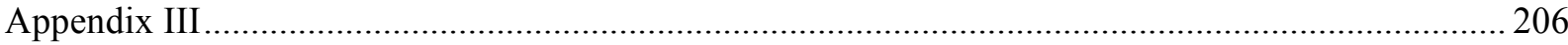

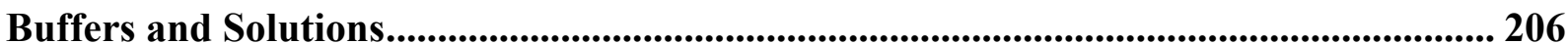

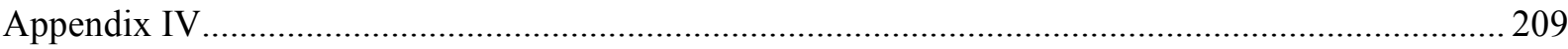


Antibodies and Dyes.

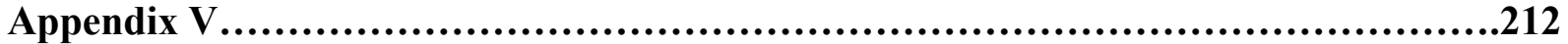

ELISA Reagents and concentrations ..... 


\section{List of Figures}

Figure 3.1 Clozapine treatment attenuates EAE disease course - day 15.............................................5

Figure 3.2 Clozapine treatment attenues EAE disease course - day 20............................................................57

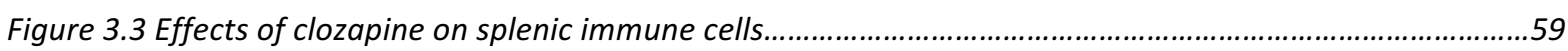

Figure 3.4 Clozapine treatment after the induction phase of EAE is not effective at reducing disease................61

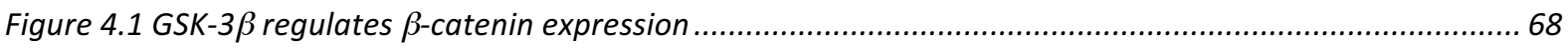

Figure 4.2 Clozapine reduced viability of stimulated CD4 T cells at concentrations greater than $40 \mu M$........... 71

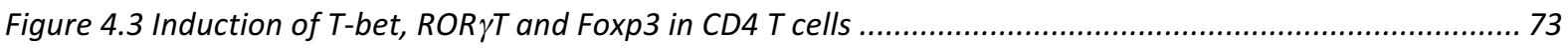

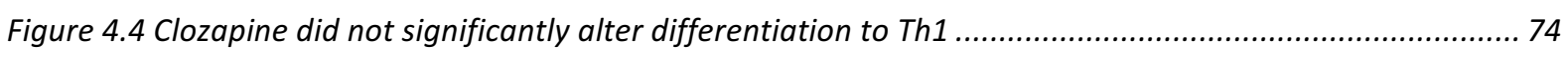

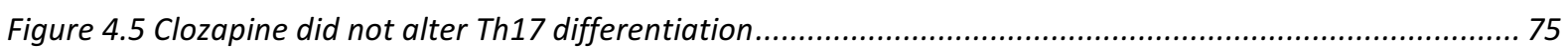

Figure 4.6 Clozapine promoted Treg differentiation and Foxp3 expression ............................................... 76

Figure 4.7 8-catenin expression was unaltered by clozapine in iTreg ......................................................... 78

Figure 4.8 Clozapine had no effect on Th development during the induction phase of EAE...............................80

Figure 4.9 Clozapine did not alter Th subset development during the effector phase of EAE .............................8 82

Figure 4.10 Clozapine did not alter $M O_{35-55}$-specific $T$ cell expansion after EAE induction ...............................84

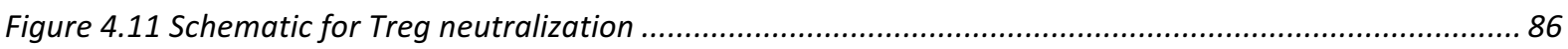

Figure 4.12 Protection by clozapine was not dependent on $C D 25^{+}$regulatory $T$ cells..................................... 88

Figure 4.13 Adoptive transfer of T cells expanded in MOG35-55-peptide with IL-12p70 and anti-IFN- $\gamma$ was

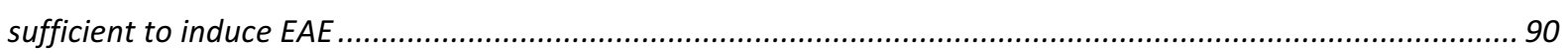

Figure 4.14 Clozapine did not alter encephalitogenicity of $M \mathrm{OG}_{35-55}$-specific $T$ cells ...................................... 91

Figure 4.15 Clozapine did not protect from adoptively transferred EAE disease .........................................92

Figure 5.1 Clozapine limits CNS infiltration of leukocytes during EAE .................................................... 102

Figure 5.2 Clozapine treatment inhibits expression of activation markers on microglia 15 d.p.i...................... 103

Figure 5.3 Clozapine inhibits expression of activation markers on infiltrating monocytes in the spinal cord 15

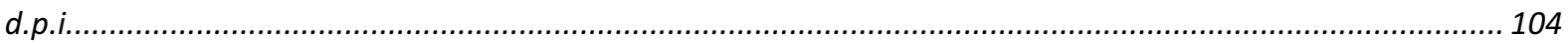

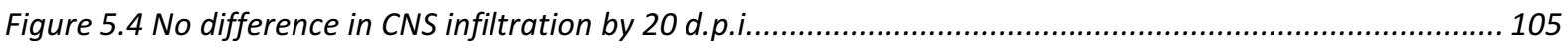

Figure 5.5 Clozapine inhibits the activation of microglia in the spinal cord 20 d.p.i ...................................... 106

Figure 5.6 Clozapine inhibits the activation of macrophages in the spinal cord 20 d.p.i................................. 107

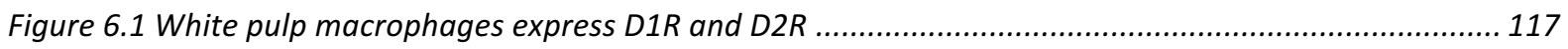

Figure 6.2 Dopamine inhibits IL-12 and increases IL-10 secretion without affecting viability........................ 118

Figure 6.3 Clozapine inhibits secretion of IL-12 and increases IL-10 in lipopolysaccharide stimulated bone

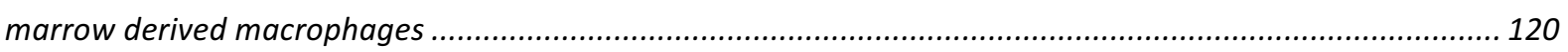

Figure 6.4 Clozapine inhibits secretion of IL-12 in lipopolysaccharide stimulated RAW264.7 cells.................. 120

Figure 6.5 Risperidone suppresses IL-12 and increases IL-10 secretion in LPS stimulated macrophages.......... 121

Figure 6.6 Forskolin inhibits secretion of IL-12 in LPS stimulated macrophages ........................................ 122

Figure 6.7 Clozapine does not alter CAMP signaling in LPS stimulated macrophages ................................. 123

Figure 6.8 Akt1/2 inhibitor increases IL-12 and decreases IL-10 secretion by macrophages ........................... 125 
Figure 6.9 Akt1/2 inhibitor is not similarly effective in bone marrow derived macrophages.

Figure 6.10 Akt1/2 inhibitor is not similarly effective in bone marrow derived macrophages at lower concentrations

Figure 6.11 CHIR99021 inhibited IL-12 and increased IL-10 secretion in bone marrow derived macrophages.. 128 Figure 6.12 CHIR99021 is not similarly effective in the RAW 264.7 cell line.

Figure 6.13 Clozapine does not alter total protein levels of several signaling proteins in response to lipopolysaccharide.

Figure 6.14 Clozapine does not alter phosphorylation of several signaling proteins involved in the response to lipopolysaccharide.

Figure 6.15 Clozapine does not alter LPS-induced phosphorylation of signaling proteins 132

Figure 6.16 Phosphorylation of NFKB p65 at s536 is not affected by clozapine 134

Figure 6.17 Potential mechanism by which clozapine is able to reduce IL-12 and increase IL-10..... 137

Figure 7.1 Levodopa/Carbidopa does not alter EAE disease progression 146

Figure 7.2 L-DOPA does not improve efficacy of clozapine during EAE 148

Figure 7.3 L-DOPA does not improve efficacy of low dose clozapine during EAE. 149

Figure 7.4 Co-administration of risperidone and fluoxetine does not improve EAE disease..... 151

Figure 7.5 Risperidone and Fluoxetine does not alter MOG-specific recall response 152 


\section{List of Abbreviations}

2D2 Mice expressing the 2D2 MOG35-55 TCR

5-HT2a Serotonin 2A

Akt Protein kinase B

ANOVA Analysis of variance

APC Antigen presenting cell

AUC Area under curve

BBB Blood-brain barrier

BCR B cell receptor

BMMØ Bone marrow derived macrophages

cAMP Cyclic Adenosine Monophosphate

CCL CC Chemokine Ligands

CD Cluster of differentiation

CFA Complete Freunds adjuvant

CFSE Carboxyfluorescein succinimidyl ester

Cloz Clozapine

CNS Central nervous system

CREB Cyclic AMP response element binding protein

CTCM Complete T cell medium

CTLA-4 Cytotoxic T lymphocyte associated protein 4

DAMP Danger associated molecular pattern

d.p.i Days post immunization

D2 Dopamine D2 receptor

DC Dendritic cell

dH20 Distilled water

dLN Draining lymph node

DMSO Dimethyl Sulfoxide

DNA Deoxyribonucleic acid

dPBS Dulbecco's Phosphate buffered saline

EAE Experimental autoimmune encephalomyelitis

EDTA Ethylenediaminetetraacetic acid

ELISA Enzyme linked immunosorbent assay

ERK Extracellular signal-regulated kinase

FCS Foetal calf serum

FLUOX Fluoxetine

Foxp3 Forkhead box p3

FSC-A Forward scatter area

FSC-H Forward scatter height

g Gravity

GATA3 GATA binding protein 3

GM-CSF Granulocyte-macrophage colony-stimulating factor

GPCR G-protein coupled receptor

GSK-3 Glycogen synthase kinase-3

HC Healthy control

I-CAM1 Intercellular adhesion molecule 1

i.p Intraperitoneal

IBMX 3-isobutyl-1-methylxanthine 


\begin{tabular}{|c|c|}
\hline IFN & Interferon \\
\hline $\operatorname{Ig}$ & Immunoglobulin \\
\hline $\operatorname{IgG}$ & Immunoglobulin $\mathrm{G}$ \\
\hline IL- & Interleukin \\
\hline Itk & Interleukin 2 inducible $\mathrm{T}$ cell kinase \\
\hline iTreg & induced Treg \\
\hline JNK & c-Jun N-terminal kinase \\
\hline L-DOPA & L-3,4-dihydroxyphenylalanine \\
\hline LFA-1 & Lymphocyte function-associated antigen 1 \\
\hline LPS & Lipopolysaccharide \\
\hline MAPK & Mitogen activated protein kinase \\
\hline MFI & Mean fluorescence intensity \\
\hline MG & Microglia \\
\hline MHC & Major Histocompatibility Complex \\
\hline MIMR & Malaghan Institute of Medical Research \\
\hline MO & Monocytes \\
\hline MOG & Myelin oligodendrocyte glycoprotein \\
\hline MS & Multiple sclerosis \\
\hline mTOR & Mammalian target of rapamycin \\
\hline MTT & 3-(4,5-Dimethylthiazol-2-yl)-2,5-Diphenyltetrazolium \\
\hline $\mathrm{NF} \kappa \mathrm{B}$ & Nuclear factor kappa-light chain enhancer of activated B cells \\
\hline NO & Nitric oxide \\
\hline NS & not significant \\
\hline OD & Optical density \\
\hline Olanz & Olanzapine \\
\hline PAMP & Pathogen associated molecular patterns \\
\hline PBMC & Peripheral blood mononuclear cell \\
\hline PBS & Phosphate buffered saline \\
\hline $\mathrm{PE}$ & Phycoerythrin \\
\hline PFA & paraformaldehyde \\
\hline PI3K & Phosphatidylinositol-4,5-bisphosphate 3-kinase \\
\hline PLC & Phospholipase C \\
\hline PMA & Phorbol 12-myristate 13-acetate \\
\hline PPMS & Primary progressive multiple sclerosis \\
\hline PRR & Pattern recognition receptor \\
\hline PTx & Pertussis toxin \\
\hline $\mathrm{rIgG}$ & Rat Immunoglobulin G \\
\hline Risp & Risperidone \\
\hline $\mathrm{ROR} \gamma \mathrm{T}$ & Retinoic acid receptor related orphan receptor $\gamma \mathrm{T}$ \\
\hline ROS & Reactive oxygen species \\
\hline RRMS & Relapsing-remitting multiple sclerosis \\
\hline RT & Room temperature \\
\hline s.c & Subcutaneous \\
\hline SA-HRP & Streptavidin-horseradish peroxidase \\
\hline SEM & Standard error of mean \\
\hline SLAM & Signalling lymphocytic activation molecule \\
\hline SMAD & Mothers against decapentaplegic homolog \\
\hline SPMS & Secondary progressive multiple sclerosis \\
\hline SSC-A & Side scatter area \\
\hline
\end{tabular}


SSRI Selective serotonin re-uptake inhibitor

STAT3 Signal transducer and activator of transcription 3

STAT5 Signal transducer and activator of transcription 5

T-bet T-box transcription factor expressed in $\mathrm{T}$ cell

TCR T cell receptor

TGF Transforming growth factor

Th $\quad$ T helper cell

Th1 T helper type 1 cell

Th17 T helper type 17 cell

Th2 T helper type 2 cell

TLR Toll-like receptor

TMB 3,3',5,5'-Tetramethylbenzidine

TNF Tumour necrosis factor

Treg Regulatory $\mathrm{T}$ cell

VCAM1 Vascular cell adhesion molecule 1

VLA-4 Very late antigen-4

VUW Victoria University of Wellington

B2-AR Beta 2 adrenergic receptor 


\section{Chapter 1: Introduction}




\subsection{Immunity}

The mammalian body is protected from infectious and pathogenic organisms by a variety of different cells and organs that make up the immune system. To protect from potentially damaging organisms, the immune system must be able to identify and respond to the presence of a pathogen and rapidly control it. The early response to conserved pathogen structures is initiated by cells of the innate immune response through the use of germ-line encoded receptors for rapid identification (Takeuchi \& Akira, 2010). However, due to the relatively high rate of evolution in micro-organisms, the immune response must be able to adapt to respond to new and unfamiliar pathogens. The adaptive immune response has the capacity to develop highly specific pathogen recognition mechanisms and memory, allowing for a more specific response and efficient response during subsequent infections. Both the innate and adaptive immune responses work together to eradicate infection and have remarkable capacity to recognize an extraordinary number and variety of structures that can be found on invading organisms; as a consequence, they are also able to recognize structures from the host itself. Highly efficient checkpoints and regulatory mechanisms are in place to prevent the immune system from recognizing host tissue as foreign and maintaining immune tolerance; however, some selfreactive cells may escape self-tolerance mechanisms and cause inflammatory damage to host tissue. This is known as autoimmunity.

\subsection{The innate immune response}

For an individual to be protected against infectious diseases, the immune system must be able to recognize the presence of an infection as quickly as possible to minimize the spread and replication of the pathogen. This response is initiated by the innate immune system, which is capable of recognizing conserved patterns commonly found on micro-organisms like bacteria, parasites and fungi. These patterns are pathogen associated molecular patterns (PAMP) and are recognized by a wide range of germ line encoded pattern recognition receptors (PRR) expressed on several different immune cells of the innate immune system, namely, macrophages, monocytes, mast cells, dendritic cells and granulocytes. PAMP are generally required for the survival of invading micro-organisms and are therefore highly conserved among micro-organisms. PRR evolved to recognize those conserved structures on microorganisms that are not expressed in host tissue such as lipopolysaccharides (LPS) in gram negative bacteria (Takeuchi \& Akira, 2010). 
Activation of these receptors results in a cell signalling cascade that ultimately causes the secretion of molecules to induce inflammation and recruit other immune cells, directing the immune response. Toll-like receptors (TLR) are an important class of PRR and are collectively able to recognize structures on a wide range of bacteria, fungi, parasites, viruses and even molecules derived from host cells. The activation of each of these receptors results in distinct signalling and gene expression that drives the activation of the innate immune system and the type of adaptive immune response that is required to eliminate the pathogen (Akira $\&$ Takeda, 2004).

Dead or dying cells release damage associated molecular patterns (DAMP) such as DNA, RNA, ATP, uric acid and heparin sulphate that are also recognised by PRR and like PAMP, are able to initiate or enhance an immune response to damage or infection (Tang, Kang, Coyne, Zeh, \& Lotze, 2012). However, in some cases this may also lead to aggravated autoimmune inflammation like in systemic lupus erythematosus and rheumatoid arthritis (Land, 2015).

In addition to pathogen recognition, a group of innate immune cells are capable of phagocytosis; the ability to engulf micro-organisms, dead cells and other debris (Underhill \& Goodridge, 2012). Cells that are capable of phagocytosis like macrophages and dendritic cells are often known to be able to process antigens derived from ingested micro-organisms and present them to the adaptive immune system, generating and directing the adaptive immune response with specificity for the antigen that is being presented (Iwasaki \& Medzhitov, 2015). Antigen presentation by antigen presenting cells (APC) like dendritic cells and macrophages produce an array of cytokines to send signals in an autocrine, paracrine and endocrine manner that can direct adaptive immune responses and general inflammation.

Granulocytes such as neutrophils, eosinophils and basophils are components of the innate immune system and play important parts in the immune response to invading pathogens, particularly during parasitic infections due to their killing properties and ability to promote humoral immunity (Cadman \& Lawrence, 2010). Granulocytes are released from the bone marrow to circulate in the blood where they migrate into tissues under inflammatory conditions. Granular in nature, the granulocytes are highly capable killers as they are able to rapidly secrete antimicrobial factors stored in intracellular granules. Neutrophils are one of the key cell types involved in innate immunity to infection. They response rapidly to infection and are highly capable killers, but have a short lifespan of only 6-8 hours (Summers et al., 2010). 
Monocytes continually circulate in the blood stream and enter tissues during an insult such as an infection, mature into macrophages and are crucial for clearing pathogens from the body. In the absence of infection, tissue-resident macrophages populate most tissue such as microglia in the central nervous system (CNS). These tissue-resident macrophages do not arise from blood derived monocytes but develop early during foetal development, primarily from yolksac derived erythro-myeloid progenitors (Gomez Perdiguero et al., 2015). Tissue-resident macrophages play important roles in tissue homeostasis by responding to infection, clearing debris, promoting wound repair and even contributing to the regulation of tissue metabolism (Wynn, Chawla, \& Pollard, 2013). As a consequence, macrophages can also contribute to disease, driving the damaging inflammatory process in many autoimmune and inflammatory diseases such as multiple sclerosis (Bogie, Stinissen, \& Hendriks, 2014; Krausgruber et al., 2011).

\subsection{The adaptive immune response}

While innate immune cells are the first responders to infection, they can only react to conserved patterns and utilize non-specific defence mechanisms. Due to the high rates of evolution, diversity and mutation in micro-organisms, the immune system must be able to adapt to changes rapidly. This task is performed by the adaptive immune system, primarily consisting of lymphocytes. Lymphocytes consist of $\mathrm{B}$ and $\mathrm{T}$ cells which produce highly specific antibodies while $\mathrm{T}$ cells provide antigen specific cell mediated immunity, either by perpetuating the immune response or direct cytotoxic function. Lymphocytes achieve this task effectively and with high specificity by randomly generating highly variable and specific antigen receptors on both $\mathrm{B}$ lymphocytes and $\mathrm{T}$ lymphocytes. This process in theory, can generate a colossal $1 \times 10^{20}$ possible combinations (Lieber, 1991) but of course, many of the randomly generated antigen receptors are non-functional or have potential to be self-reactive; therefore selection mechanisms are in place to delete auto-reactive cells. T cells that express $\mathrm{T}$ cell receptors which bind to self-MHC molecules that triggers a TCR signal exceeding a certain "threshold" are deleted during negative selection to prevent potentially auto-reactive TCRs from escaping into the periphery. In contrast, T cells that express a TCR that is unable recognise MHC and generate a signal undergo death by neglect as they do not receive the survival signals required for maturation and survival (Klein, Kyewski, Allen, \& Hogquist, 2014). 
Lymphocytes expressing functional but non-self-reactive antigen receptors are released into the peripheral repertoire and thus the actual number of unique receptors is $2 \times 10^{6}$ in mice (Casrouge et al., 2000) and $2 \times 10^{7}$ in humans for $\mathrm{T}$ cells (Miles, Douek, \& Price, 2011). Lymphocytes mature from a common lymphoid progenitor in the bone marrow. Whereas B cells mature and develop highly specific B cell receptors in the bone marrow (LeBien \& Tedder, 2008), T cells migrate to the thymus where they undergo interrogation of their antigen receptors, a process called positive and negative selection, before entering the circulation (Klein et al., 2014). Additionally, the process of thymic selection and the signal strength each $\mathrm{T}$ cell receives can influence its developmental pathway. For example, $\mathrm{T}$ cells that receive a strong TCR signal that does not exceed the threshold for negative selection may differentiation into natural Treg (nTreg) (Hsieh, Lee, \& Lio, 2012).

\subsubsection{Antigen recognition by $T$ cells}

T cell antigen receptors or T cell receptors (TCR) recognize antigens only as part of a complex with a self major histocompatibility complex (MHC) molecule. Therefore, as part of the selection process, TCR must be able to recognize self-MHC molecules in complex with a peptide. There are two classes of MHC molecules and both consist of two polypeptide chains, the MHC Class I and II; both serving distinct functions. MHC Class I molecules are made up of an $\alpha$ chain and a $\beta 2$-microglobulin (Vitiello, Potter, \& Sherman, 1990). Typically, MHC Class I molecules present peptides from intracellularly derived antigens i.e viral proteins produced by the host cell during viral infection. These cells acquire help from cytotoxic $\mathrm{T}$ cells by interacting with their TCR and CD8 co-receptor through MHC Class I to eliminate the cells harbouring viruses .

MHC Class II molecules are made up of an $\alpha$ and a $\beta$ chain, and typically present antigens that is derived extracellularly. i.e phagocytosis of a bacterium. MHC Class II molecules interact with CD4 on helper T cells that provide help signals for eliminating the pathogen as well as activating the adaptive immune response (Neefjes, Jongsma, Paul, \& Bakke, 2011). Both MHC molecules are highly polymorphic at the area of peptide binding to allow the loading of peptides from a vast array of sources. Conventional T cells that are MHC restricted consist of $\alpha$ and $\beta$ polypeptide chains linked by a disulphide bond. The $\alpha$ and $\beta$ chains consist of two domains that resemble the variable $(\mathrm{V})$, joining $(\mathrm{J})$ and constant $(\mathrm{C})$ domains of immunoglobulins. The $\beta$ chain also has a diversity (D) gene segment (Nemazee, 2006). Each of these domains are 
encoded by multiple gene segments in the genome and are randomly combined to form a TCR repertoire that is highly polymorphic and has potential to create over $1 \times 10^{20}$ different combinations of $\alpha \beta$ TCR (Lieber, 1991).

\subsubsection{Antigen recognition by $B$ cells}

In contrast to TCR, B cell receptors (BCR) have two antigen binding sites and can be secreted as antibody (i.e. immunoglobulin; Ig). Unlike TCR, BCR or antibodies can bind directly to an antigen and does not require presentation by MHC. Like T cells, an enormous collection of B cells develops in the immune system such that they are collectively able to recognize a vast array of foreign bodies and molecules. Immunoglobulins are structured proteins composed of an antigen-binding region called the variable region (V) and a constant region $(\mathrm{C})$ that allows recognition by host immune cells. Like T cells, B cells create an enormous immunoglobulin repertoire by genetic re-arrangement of the $\mathrm{V}$ region coded by their immunoglobulin genes. In addition, B cells further mature and develop highly specific antibodies and receptors by somatic hypermutation, which randomly introduces point mutations in the BCR. By natural selection, BCR with higher affinity are selected into the repertoire. High affinity BCR can then be secreted by B cells to provide circulating antibodies that act as the first line of defence to subsequent infections or insults (Kurosaki, Kometani, \& Ise, 2015).

\subsubsection{T helper cell subsets}

Helper T cells interact with MHC Class II via their CD4 receptor. Activated helper T cells direct immune responses by producing a diverse and ordered set of cytokines, each set specific for the type of immune response. In addition, helper $\mathrm{T}$ cells activate $\mathrm{B}$ cells and also regulate the magnitude of an immune response. T helper cells achieve these tasks by differentiating into distinct effector $\mathrm{T}$ cell subsets, the core group of which consists of Th1, Th2, Th17 and regulatory T cells (Treg) (Harrington et al., 2005; Mosmann \& Coffman, 1989; Wan, 2010).

Th1 cells mount an immune response that is efficient for eliminating intracellular bacteria, viruses and even cancer cells. Th1 cell development is controlled by the master regulatory transcription factor T-bet when $\mathrm{T}$ helper cell activation occurs in the presence of IL-12 (Lazarevic, Glimcher, \& Lord, 2013), typically produced by activated APC. Th1 cells produce IFN- $\gamma$ to promote inflammatory responses in innate immune cells making them more effective at eliminating intracellular pathogens including viruses. As a mechanism of preventing an 
inappropriate immune response to an infection, IFN- $\gamma$ negatively regulates the development of other $\mathrm{T}$ cell subsets like Th2 as these may not be useful during intracellular infections. In addition, Th1 cytokines promote cytotoxic $\mathrm{T}$ cell responses to viruses, bacteria and even host cells. Due to these functions, Th1 cells are implicated in many autoimmune and inflammatory disorders such as multiple sclerosis (Fletcher, Lalor, Sweeney, Tubridy, \& Mills, 2010) and type I diabetes (Phillips et al., 2009; Walker \& von Herrath, 2016).

Th2 cells generate a different functional response which is better suited for the clearance of extracellular pathogens and parasites. They promote antibody responses and effector functions of granulocytes and consequently are involved in the pathological mechanisms of allergy and asthma (H. Y. Kim, DeKruyff, \& Umetsu, 2010; Paul \& Zhu, 2010). The transcription factor GATA3 regulates the development of Th2 cells from IL-4 signalling (Ho, Tai, \& Pai, 2009). Th2 cells produce IL-4, IL-5, IL-10 and IL-13 which promote a humoral immune response that is less harmful in an autoreactive response and limits the extent of harmful Th1 immune responses (Wynn, 2015).

Th17 cells were a more recently discovered Th cell subset and are distinct from Th1 and Th2 cells by producing IL-17A and GM-CSF. These cytokines are under the control of the master regulator of Th17 cells, ROR $\gamma$ T (Codarri et al., 2011; Ivanov et al., 2006) and is now used as a marker to differentiate this lineage from other T helper subsets. Unlike Th1 and Th2 cells, Th17 cells differentiate in the presence of IL- 6 and TGF- $\beta$ which induces the expression of ROR $\gamma \mathrm{T}$ (Ivanov et al., 2006). Th17 cells support neutrophil responses and like Th2 cells, are important for clearance of extracellular bacteria and parasites. However, they also have tissue damage promoting functions and are key players in autoimmune and inflammatory disease like multiple sclerosis (Fletcher et al., 2010).

One of the most important subsets in maintaining immune homeostasis are regulatory $\mathrm{T}$ cells (Treg). They have a very different role to many of the other Th subsets and have immunosuppressive qualities to limit potential damage to host tissues (Yamaguchi, Wing, \& Sakaguchi, 2011) by excessive inflammatory response or self-reactive T cells which may have escaped negative selection in the thymus (Wing \& Sakaguchi, 2010). Several subsets of regulatory $\mathrm{T}$ cells have been described. The most common and most studied are the CD4+ and CD8+ Treg. Development of this unique phenotype is controlled by the transcription factor Foxp3, which is required for their regulatory properties (Hori, Nomura, \& Sakaguchi, 2003). Loss of the Foxp3 gene in mice results in global autoimmunity (J. M. Kim, Rasmussen, \& 
Rudensky, 2007) highlighting the key role Treg play in maintaining immune tolerance. It is noteworthy to mention that more recently, a new subset of regulatory cells were discovered. These were immunosuppressive B cells (Fillatreau, Sweenie, McGeachy, Gray, \& Anderton, 2002) and are named regulatory B cells or Breg (Rosser \& Mauri, 2015).

\subsubsection{Cytotoxic $T$ cells}

In a similar manner to $\mathrm{T}$ helper cells, cytotoxic $\mathrm{T}$ cells interact with MHC Class I through their TCR and CD8 receptor. MHC Class I molecules present antigens that are endogenous to the cell such as abnormal or virally encoded proteins. Presentation of these antigens typically indicates an abnormal or virally infected cell that must be eliminated to prevent replication or spread. This task is performed by the CD8 $\mathrm{T}$ cell, which produces cytotoxic granzyme and perforin to kill cells directly. In addition, CD8 T cells can induce apoptosis by Fas ligand interactions (Barry \& Bleackley, 2002).

Antigens presented on MHC Class I are typically derived from endogenous antigens that have been processed through the proteasome and are indicative of viral infection, cellular damage or mutation (Hansen \& Bouvier, 2009). In contrast, antigens presented on MHC Class II are exogenously derived by endocytosis and are loaded onto MHC Class II (Roche \& Furuta, 2015). In general, exogenously derived antigens cannot be loaded onto MHC Class I and endogenously derived peptides cannot be loaded onto MHC Class II as they do not share the same processing pathway. However, antigen presenting cells, particularly dendritic cells have a specialised function called cross-presentation which allows endogenously loaded antigens to be presented onto MHC Class I . Cytotoxic T cells are also capable of being activated by exogenous ligands through a process of cross-presentation by dendritic cells (Milo et al., 2013).

Unfortunately, like CD4 T cells, CD8 T cells develop their TCR in the same manner and are susceptible to recognizing self-antigens. Failure of self-tolerance mechanisms can lead to CD8 mediated autoimmunity such as type I diabetes where insulin producing $\beta$-cells in the pancreas are destroyed by CD8 T cells (Bulek et al., 2012; Pinkse et al., 2005; Toma et al., 2005). In addition, while CD4 T cells are the major contributors to diseases like MS, CD8 T cells are also known to promote disease, although their role is less clear (Huseby, Huseby, Shah, Smith, \& Stadinski, 2012). 


\subsubsection{The lymphatic system}

Because only a few lymphocytes expressing a certain antigen receptor are circulating at any one time, the chances of a T cell encountering an APC carrying its cognate antigen in the body are relatively slim. Thus, this process is made more efficient by the lymphatic system which consists of vessels that transport interstitial fluids or lymph from tissues back into the circulatory system. Around 450 lymph nodes are scattered throughout the body interconnected by lymphatic vessels, and these are the sites in which T cells and APC meet after leaving tissue (Girard, Moussion, \& Forster, 2012). Typically, when an antigen-presenting cell such as a dendritic cell encounters an antigen in peripheral tissue, DC migrate out of the tissue through lymphatic vessels and into lymph nodes where they can encounter naïve $\mathrm{T}$ cells that sample their antigens for a cognate peptide/MHC complex. Naïve T cells survey lymph nodes in the body by trafficking across sphingosine-1-phosphate (S1P) gradients. Activated T cells migrate out of lymph nodes towards an S1P gradient (Maceyka \& Spiegel, 2014). This feature of T cell activation in lymph nodes has been targeted as a therapeutic option for limiting $\mathrm{T}$ cell mediated diseases. Indeed, treatment with Fingolimod inhibits the trafficking of T cells towards the S1P gradient, retaining activated $\mathrm{T}$ cells in the lymph nodes and limiting relapse in RRMS patients (Aktas, Kury, Kieseier, \& Hartung, 2010).

\subsection{Leukocyte trafficking}

Immune cells such as lymphocytes and dendritic cells are incredibly mobile and traffic from the blood or lymphatic vessels into lymph nodes and tissue by a process called extravasation. Firstly, during an inflammatory response, leukocytes are activated by PAMPs or DAMPS leading to the production and secretion of pro-inflammatory cytokines and chemokines. These inflammatory mediators released from the site of infection or damage activates nearby endothelial cells that line the blood vessels to express E-selectin and P-selectin. These selectins mediate the transient attachment of leukocytes to the endothelium and escape the flow of blood (McEver, 2015). This transient interaction enables leukocytes to roll along the endothelium and is guided by inflammatory cytokines and chemokines. Leukocytes then activate integrin molecules that attach to VCAM1 and ICAM1 molecules. This interaction enables the transmigration of leukocytes in between endothelial cells and into the inflamed tissue. Specifically, in EAE it has been shown that the $\alpha 4 \beta 1$-integrin mediates the attachment of inflammatory lymphocytes to VCAM-1 on the inflamed brain endothelium (Lobb \& Hemler, 1994) and that blocking this integrin with specific monoclonal antibodies limits EAE disease 
(Kent et al., 1995). This treatment has later proven to be effective in RRMS and reduced relapse rate up to $68 \%$ (Polman et al., 2006).

\subsection{Antigen presentation - bridging the innate and adaptive immune response}

Antigen presentation by MHC Class I and II molecules are absolutely essential for activation of $\mathrm{T}$ cells as their antigen receptors only recognize antigen in the context of MHC. The most competent APC of the immune system are the dendritic cells and macrophages. Dendritic cells are known as professional APC as they are continually sampling antigens from the periphery and presenting antigens to T cells in lymphatic organs. Antigens derived from the proteasome in the cytoplasm (i.e viral or cancerous antigens) are presented on MHC Class I whereas antigens that undergo proteolysis in endosomes and lysosomes are generally presented onto MHC Class II (Neefjes et al., 2011).

Mature $\mathrm{T}$ cells that have not yet recognized a specific antigen are known as naïve $\mathrm{T}$ cells. Naïve $\mathrm{T}$ cells circulate from the blood through lymphoid tissues to survey antigen-presenting cells that have migrated to a lymphoid tissue. Once an antigen-presenting cell like a dendritic cell (DC) encounters an antigen, the DC migrates to a lymphoid tissue and presents the antigen in complex with a major histocompatibility complex (MHC) to a naïve $\mathrm{T}$ cell expressing a complementary TCR which initiates a signalling cascade inducing $\mathrm{T}$ cells to proliferate and become effector cells (Zhu, Yamane, \& Paul, 2010). This signalling cascade recruits the key signalling protein phospholipase $\mathrm{C}-\gamma(\mathrm{PLC}-\gamma)$ to the membrane, however, PLC- $\gamma$ requires activation by the tyrosine kinase Itk on the membrane (Smith-Garvin, Koretzky, \& Jordan, 2009). This task is performed by ligation of CD28 from co-stimulatory molecules such as CD80 and CD86 expressed by APCs like DC (Lenschow, Walunas, \& Bluestone, 1996). During an infection or inflammatory response, APC up-regulate the expression of CD80 and CD86 molecules (F. Zhou et al., 2012). CD28 on the T cell are part of the immunological synapse formed when TCR interact with an APC and activates another signalling cascade which leads to the phosphorylation of Itk and then allows PLC- $\gamma$ to become fully activated and enhance T cell activation (Acuto \& Michel, 2003). In addition, T cells can also express and upregulate co-inhibitory molecules such as cytotoxic T lymphocyte antigen-4 (CTLA-4) after activation that also binds and competes for CD80 to inhibit inflammatory responses (Alegre, Frauwirth, \& Thompson, 2001). Therefore, co-stimulation has a critical role in determining the 
outcome of $\mathrm{T}$ cell activation. $\mathrm{T}$ cells differentiate depending on the type of signals provided during priming and this interaction has capacity to direct inhibition, activation, differentiation and the survival of $\mathrm{T}$ cells; directing an effective immune response without damaging host tissue.

Co-stimulatory molecules also direct the differentiation of $\mathrm{T}$ cells into different $\mathrm{T}$ cell subsets. For example, SLAM molecules expressed by APC promote the production of IL-4 from T cells during activation (Cannons, Tangye, \& Schwartzberg, 2011) whereas CD27 promotes the differentiation of Th1 cells (van Oosterwijk et al., 2007). APC further control the T cell response by the cytokines produced during activation as previously discussed in this chapter. Finally, recent studies have demonstrated that APC encountering bacterial antigens drive an IFN- $\gamma$-producing Th1 response (W. S. Kim et al., 2016) whereas APC that encounter parasite derived antigens drive IL-4-producing Th2 cells (Connor, Tang, Camberis, Le Gros, \& Ronchese, 2014), highlighting the importance of the APC in directing effective $\mathrm{T}$ cell responses.

\subsection{Immunological tolerance}

In order for the immune system to recognize foreign entities that have never been encountered, the immune system must be able to recognize almost every chemical structure imaginable. To achieve this, the adaptive immune system must be able to create a diverse set of antigen receptors, namely, the BCR and TCR. The mammalian immune system generates a diverse repertoire of BCR and TCR through a process of random somatic recombination of the variable (V), diversity (D) and joining (J) gene segments (Schatz \& Ji, 2011). In theory, this genetic recombination can create up to $10^{20}$ different TCR allowing for astounding ability to recognize chemical moieties from all sorts of foreign entities. Around $90 \%$ of these die due to a lack in TCR signalling as they are essentially useless TCRs (Lu et al., 2000). The random nature of T cell and B cell development, between $20 \%$ and $50 \%$ of these have the potential to recognize host antigens. Therefore, the immune system must have regulatory checkpoints to maintain immunological tolerance of host tissue. To achieve this, lymphocytes undergo multiple regulatory barriers. Firstly, lymphocytes bearing self-reactive receptors are deleted by inducing apoptosis, a process called negative selection. Secondly, B cells can undergo receptor editing to become less reactive or become anergic and finally, self-reactive lymphocytes can become regulatory cells to limit their auto-reactivity even though they are present in the peripheral tissue (Luning Prak, Monestier, \& Eisenberg, 2011). Although these are efficient processes, 
some auto-reactive cells do escape the selection process in the thymus and circulate in the periphery. These are normally kept under control by peripheral tolerance mechanisms of Tregs, which are also auto-reactive but have potent immunosuppressive properties (Vignali, Collison, \& Workman, 2008). Altogether, these mechanisms are highly effective at controlling selftolerance with only $3 \%$ of the human population developing an autoimmune disease. Failure of these regulatory mechanisms in autoimmune disease eventuates from genetic predisposition, usually associated with MHC variants (Cho \& Feldman, 2015) as well as environmental factors.

\subsubsection{Central Tolerance}

Central tolerance is established by deletion of self-reactive $\mathrm{T}$ cells that are generated from random re-arrangement of the TCR. Immature T cells enter the thymus and begin development and interrogation of their TCR by peptide:MHC interactions. Between $70-90 \%$ of $\mathrm{T}$ cells do not encounter productive peptide:MHC interactions because they are non-functional and undergo apoptosis (Lu et al., 2000). When T cells undergo selection, they express both CD4 and CD8 co-receptors and depending on whether the TCR interacts with an MHC Class I or MHC Class II molecule determines its fate of becoming either a cytotoxic $\mathrm{T}$ cell or a helper $\mathrm{T}$ cell (Klein et al., 2014). For example, if a double positive T cell interacts with an MHC Class II molecule, the CD4 co-receptor is kept while CD8 is lost during maturation into a single positive lymphocyte. Interestingly, the affinity for the peptide:MHC interaction also dictates the fate of the $\mathrm{T}$ cell. Interactions that reach a high affinity over a certain threshold are automatically deleted or induced to become thymus-derived nTreg. These thymus-derived nTreg are allowed to escape into the periphery but are regulatory in nature and act to limit autoimmune reactions. This is an important feature of maintaining central tolerance and prevents auto-reactive $\mathrm{T}$ cells with high affinity from escaping selection.

\subsubsection{Peripheral Tolerance}

Central tolerance mechanisms are not fool proof, and some self-reactive T cells escape into the periphery. These self reactive T cells must be kept in check by regulatory mechanisms. One of the key mechanisms that the immune system regulates autoimmune responses by self-reactive $\mathrm{T}$ cells are by the function of Tregs which protect the host from inflammatory damage induced by inflammation from an infection or an autoimmune response. Tregs develop from two distinct mechanisms. Firstly, natural Tregs (nTreg) develop in the thymus during normal T cell 
development and undergo positive and negative selection. In contrast to conventional $\mathrm{T}$ cells, nTregs generally receive a stronger TCR signal during negative selection and are directed towards the nTreg lineage and express the transcription factor Foxp3. T cells that receive even stronger TCR signals are deleted (Maloy \& Powrie, 2001). Induced Tregs (iTregs) develop in the periphery from Foxp3- CD4 T cells during inflammatory responses to further control inappropriate immune responses. This subset of Treg can be induced in vitro by TCR stimulation in the presence of IL- 6 and TGF- $\beta$. Both natural and induced subsets are understood to be important for maintaining immune homeostasis (Schmitt \& Williams, 2013).

Tregs regulate the immune system and suppress inappropriate immune responses through a number of mechanisms. Firstly, when nTregs encounter a self-antigen in the periphery, they can become activated and secrete inhibitory cytokines such as IL-10 and TGF- $\beta$ to limit inflammation. In addition, these cytokines also contribute to the development of iTregs in the periphery. Tregs can also inhibit inflammatory responses from $\mathrm{T}$ cells by disrupting cellular IL-2 signaling by expressing high levels of IL-2 receptor to deplete IL-2 from effector T cells. Tregs can also have immunosuppressive effects through direct cell-cell contact. CTLA-4 interactions with CD80 and CD86 can activate the indoleamine 2,3 dioxygenase pathway in dendritic cells which can suppress T cell responses (Vignali et al., 2008). Loss of Treg function, by mutations in the FOXP3 gene cause a systemic autoimmune inflammation that is characterised by hyperproliferation of lymphocytes in both mice and humans (Ziegler, 2006). Their importance is further highlighted by studies that show Tregs are crucial for protection against diabetes, asthma, inflammatory bowel disease and other inflammatory disorders (Sakaguchi et al., 2001; Xystrakis, Boswell, \& Hawrylowicz, 2006).

\subsection{The blood-brain barrier}

The blood brain barrier separates the peripheral circulatory system from the central nervous system in order to protect the CNS from harmful toxins, pathogens and other molecules. Simultaneously, the blood brain barrier tightly controls the entry of ions, glucose and other nutrients to meet the demands of the CNS. To achieve this barrier function, the endothelial cells that line the cerebral blood continuously form tight junctions in between them, lack fenestrations and are poor at transcytosis(Abbott, Ronnback, \& Hansson, 2006). All of these features of CNS endothelial cells are in place to regulate the CNS environment; limiting the entry of nutrients, oxygen, ions, proteins, peptides and other small molecules across the blood 
brain barrier. In addition, unlike peripheral tissues, the blood brain barrier in a resting state protects the CNS from peripheral immune cell infiltration by its low resting expression of leukocyte adhesion molecules during the steady state. However, in order to protect CNS tissue from infection and other insults, the blood-brain barrier endothelial cells can express leukocyte adhesion molecules to allow the trafficking of inflammatory cells into the CNS.

The protective nature of the blood-brain barrier prevents the entry of small molecules larger than $500 \mathrm{Da}$, from the blood into the CNS (Pardridge, 2005a). This size restriction limits almost all protein or antibody based therapeutics from entering the CNS. In addition, studies have shown that the lipophilicity and ionic charge also contribute to the diffusion properties of the molecule (Pardridge, 2005b), making it extremely challenging to design effective therapeutic interventions for CNS diseases, particularly for progressive forms of MS, where the site of inflammation is trapped within an intact blood brain barrier (Lassmann, van Horssen, \& Mahad, 2012).

\subsection{Multiple Sclerosis}

MS is the most common autoimmune disease of the central nervous system affecting over 2.5 million people worldwide and around 4000 people in New Zealand. It is characterized by the presence of sclerotic plaques in the brain and spinal cord caused by the infiltration of inflammatory immune cells leading to the demyelination of neuronal axons. This demyelination impairs signal transduction, and if not repaired or remyelinated will eventually progress to accumulating cognitive and physical disability. Almost half of all MS patients will require the use of a wheelchair 25 years from initial diagnosis, posing a significant burden on the quality of life for sufferers and healthcare. Approximately $85 \%$ of diagnosed sufferers are diagnosed with relapsing-remitting MS (RRMS) typically presenting first with an acute attacks of neurological dysfunction followed by periods of remission where some of the symptoms may subside. Most commonly, the recovery periods shorten with age while disability continually accumulates. Almost 50\% of all RRMS patients will progress into secondary progressive multiple sclerosis (SPMS), where inflammatory attacks are no longer followed by periods of remission and instead enter into the progressive stage of disease in which new lesions continue to form and clinical complications accumulate without periods of recovery. Not all 
patients first present with RRMS but instead some experience progressive disease from the beginning. This type of MS is primary progressive MS (PPMS) (Lublin \& Reingold, 1996).

The cause and triggers of MS are still unknown, although multiple risk factors including genetics (Gourraud, Harbo, Hauser, \& Baranzini, 2012) and previous infections with certain pathogens have been identified (Ascherio \& Munger, 2007). A common theory among many investigators is that an autoimmune reaction is initiated against myelin antigens in the brain, resulting in the immune recognition of the myelin sheath surrounding neuronal axons as foreign by $\mathrm{T}$ cells. Thus an adaptive immune response is initiated in the CNS, recruiting inflammatory cells such as monocytes that destroy the myelin sheath (Dendrou, Fugger, \& Friese, 2015). The myelin sheaths surrounding neuronal axons are critical for signal transmission, and their damage leads to the clinically observed symptoms of MS.

This immunological theory of MS has led to the extensive use of experimental autoimmune encephalomyelitis (EAE) as an animal model for MS. EAE is induced by immunization of mice with myelin-derived antigens in an adjuvant to induce inflammatory demyelination (Constantinescu, Farooqi, O'Brien, \& Gran, 2011) that models certain aspects of MS. Use of this model by researchers has led to the development and approval of natalizumab (T. A. Yednock et al., 1992), glatiramer acetate (Teitelbaum, Meshorer, Hirshfeld, Arnon, \& Sela, 1971) and mitoxantrone (Ridge et al., 1985) for the treatment of MS. These drugs target mechanisms involved in the inflammatory response by modifying the macrophages and $\mathrm{T}$ cells known to be critical for disease.

While all clinical forms of MS are mediated by the inflammatory response, there is still much heterogeneity in the pathological mechanism. Specifically, RRMS patients normally present with impaired blood-brain barrier (BBB) integrity and subsequent infiltration of inflammatory lymphocytes and monocytes into the CNS from the periphery. In contrast, progressive forms of MS present with only mild BBB impairment and minimal infiltration of inflammatory cells from the periphery despite ongoing inflammation in the CNS (Hochmeister et al., 2006). These studies suggest that inflammation in progressive MS is sequestered to the CNS and is physically isolated from the involvement of the peripheral immune system due to minimal disruption of the BBB. Thus, perpetuation of the inflammatory response in progressive disease is attributed to microglia and macrophages and their importance make them a potentially effective target for treating CNS inflammatory disease, however, drugs that target these cells must also be able to readily cross the BBB. 
Currently, patients at the progressive stages of disease have no effective treatment options available to them as currently approved MS therapeutics are effective only in RRMS (Feinstein, Freeman, \& Lo), thus there is an urgent need for alternative therapies. This is not surprising given that current agents either target the trafficking of peripherally derived cells into the CNS like fingolimod and natalizumab; or they are incapable of crossing the BBB effectively, as the BBB prevents molecules such as beta-interferons and glatiramer acetate from entering the CNS. Therefore, in principle, therapeutic agents that can cross the BBB and modulate the inflammatory response in the CNS may offer means by which inflammatory damage and clinical disability can be minimized during the progressive forms of MS.

\subsection{Experimental autoimmune encephalomyelitis as a model for CNS inflammation}

Experimental autoimmune encephalomyelitis (EAE) is an experimental model for inflammatory demyelination in the CNS and is a commonly used as a model for MS. EAE has complex immune-mediated neuropathological mechanisms making it a highly useful and adaptable animal model for studying complex immune mechanisms in organs, especially the CNS, which involve adaptive and innate immunity.

Many clinically approved treatments for diseases such as MS have been developed on the basis of EAE. Neuroinflammation during EAE shares some similarities with inflammation associated with psychiatric disorders on the basis of the roles of chronic microglia activation in inflammatory damage. However, EAE differs from psychiatric disorder-associated inflammation in that it also involves adaptive immune responses with antigen specificity. EAE can be induced in mice by immunization with myelin peptides to induce inflammatory demyelination of axons in the CNS that leads to progressive hind-limb paralysis and cognitive damage. For studying CNS inflammation, this model is useful as it has several phases of disease. The induction phase follows the subcutaneous injection of a myelin antigen in complete Freund's adjuvant and involves the priming and activation of myelin specific $\mathrm{CD} 4^{+}$ $\mathrm{T}$ cells by APC that present the myelin peptides. After EAE induction, the effector phase consists of migration and proliferation of activated myelin-specific CD4+ T cell to the CNS. Infiltrating activated $\mathrm{T}$ cells express the cytokines IFN- $\gamma, \mathrm{GM}-\mathrm{CSF}$ and stimulatory molecules CD40L and CD28 that promote activation and accumulation of resident microglia and macrophages in the CNS (Strachan-Whaley, Rivest, \& Yong, 2014). mediating inflammatory demyelination of neuronal axons ultimately contributing to neurodegeneration and damage in the brain (S. D. Miller, Karpus, \& Davidson, 2007). 


\subsection{Microglia}

In homeostatic conditions, microglia are quiescent and are poor APC due to the low expression of MHC class II and co-stimulatory molecules whereas peripherally-derived monocytes generally express higher levels and are more readily induced to respond. During the induction phases of EAE, microglia become active and gain the capacity to act in a way that is similar to inflammatory macrophages in the periphery. Firstly, activated microglia in the induction phase of EAE adopt a type I phenotype and quickly acquire the capacity to secrete the chemokines CCL2, CCL3, CCL4, CCL5 and CCL22 to attract inflammatory cells to the CNS (Mildner et al., 2009; Mizutani et al., 2012). Microglia can also be type II activated and secrete less inflammatory cytokines and more IL-10 (Stone \& La Flamme, 2016) suggesting that the activation state is dependent upon the microenvironment.

In EAE activation of microglia occurs early, prior to the entry of peripherally-derived cells suggesting that they are crucial for permitting entry and recruiting inflammatory cells. Preventing the activation of microglia or impaired signalling by these chemokines dampens CNS infiltration and severely delays and reduces EAE disease (Dogan, Elhofy, \& Karpus, 2008; Forde, Dogan, \& Karpus, 2011; Heppner et al., 2005). In addition, inflammatory microglia are able to act as APC and produce the inflammatory mediators TNF- $\alpha$, IL-6, IL-12, IL-23 and IL-1 $\beta$ in a similar manner to peripherally-derived macrophages that support the cytokine environment required for maintenance of damaging Th1 and Th17 cells. The function of microglia in the initiation and maintenance of EAE are supported by experiments that demonstrate a reduction and delay in the disease of EAE mice when microglia are inhibited or depleted (Heppner et al., 2005).

\subsection{Monocytes}

It is understood that EAE disease initiates by the activation of myelin-specific Th1 and Th17 cells, followed by the activation of CNS resident microglia that recruit peripherally-derived monocytes from the blood (Ajami, Bennett, Krieger, McNagny, \& Rossi, 2011). Depletion of monocytes or limiting their entry to the CNS suppresses EAE highlighting the importance of monocytes in mediating damage (Brosnan, Bornstein, \& Bloom, 1981; Huitinga, van Rooijen, de Groot, Uitdehaag, \& Dijkstra, 1990). Recruitment of monocytes to inflammatory sites is a natural response and can help to repair and restore tissue health (Shechter et al., 2013), but in inflammatory conditions like MS, infiltrating monocytes can aggravate the inflammatory 
response and damage CNS tissue (Ajami et al., 2011; Bruck et al., 1996). Monocytes are prominent in MS lesion formation and remain in the CNS during the progressive stages of MS where active recruitment of T cells is absent (Prineas et al., 2001). These macrophages appear to have an inflammatory phenotype (D. Y. S. Vogel et al., 2013) and express higher levels of the co-stimulatory molecules CD80 and CD86 (Kouwenhoven, Teleshova, Özenci, Press, \& Link, 2001). These monocytes mediate demyelinating damage to neuronal axons by producing tissue-damaging free radicals, proteases and inflammatory cytokines (Manoj K. Mishra \& V. Wee Yong, 2016).

Like microglia, other macrophages are also capable of exhibiting a type I inflammatory or type II regulatory response where type I macrophages are characterized by IL-12 and TNF- $\alpha$ whereas type II macrophages produce IL-4 and IL-10 and have been shown to reduce EAE disease (La Flamme et al., 2012; Tierney, Kharkrang, \& La Flamme, 2008). It is therefore suggested that some of the effective treatments for MS are beneficial for their ability to modulate macrophage responses. For example, IFN- $\beta$ alters the expression of co-stimulatory molecules CD80, CD86 and CD40 with potential to be beneficial in MS (Marckmann et al., 2004). Moreover, glatiramer acetate is a commonly prescribed drug for relapsing remitting MS and is known to induce a regulatory phenotype in myeloid cells (Weber et al., 2004). Adoptive transfer of glatiramer acetate-induced regulatory myeloid cells into EAE mice is therapeutic (Weber et al., 2007), further highlighting their twin roles in protection and pathogenesis. As macrophages can also have protective roles, it has been suggested that modulating their activation towards a less inflammatory and more regulatory phenotype can be beneficial for inflammatory diseases like MS (Manoj K. Mishra \& V. Wee Yong, 2016).

\subsection{Atypical antipsychotic agents and inflammation}

Earlier generation antipsychotics or "typical" antipsychotics such as haloperidol are dopamine D2 receptor antagonists and are effective at reducing delusions and hallucinations in psychosis patients. However, due to their mechanism of action these typical antipsychotics have a high incidence of extrapyramidal symptoms (EPS) like parkinsonism and other movement disorders (Leucht, Wahlbeck, Hamann, \& Kissling, 2003). The antagonist action of antipsychotic agents on the dopamine D2 receptor was widely accepted as the mechanism of action for reducing psychosis and no clinically available antipsychotic agent currently exists that does not have this property. As new and improved therapeutics emerge such as the atypical antipsychotic agents, we have learnt that while the antagonist effect on the D2 receptor is important for 
treating some symptoms of psychosis, unique mechanisms of action acquired by newer generation agents are more effective for treating other symptoms of psychosis and significantly reducing the incidence of EPS, thus improving therapy. While neurotransmitters were previously understood to only be molecules of communication between neurons, more recent studies have since shown that other cells like immune cells are also regulated by neurotransmitters (Levite, 2016; R. Pacheco, Riquelme, \& Kalergis, 2010). Studies have since shown that immune cells like $\mathrm{T}$ cells and macrophages express many neurotransmitter receptors including all of the D1 to D5 receptors, which indicates that they are potential targets of antipsychotic medication (McKenna et al., 2002).

Indeed, it has been shown in several studies that clozapine and risperidone are able to directly alter the activation of immune cells. Specifically, it has already been shown that clozapine protects neurons from inflammation induced by LPS. Clozapine attenuated the LPS induced production of reactive oxygen species (ROS), nitric oxide (NO) and TNF- $\alpha$ from microglia (X. $\mathrm{Hu}$ et al., 2012). This has been demonstrated in vivo when LPS injected mice were treated with atypical antipsychotic agents risperidone and clozapine as well as the typical, older generation antipsychotic haloperidol. Serum concentrations of TNF- $\alpha$ decreased dramatically with clozapine and risperidone treatment, but not haloperidol while serum concentrations of IL-10 were also increased with clozapine and risperidone while only a modest increase was measured with haloperidol (Sugino, Futamura, Mitsumoto, Maeda, \& Marunaka, 2009). These results indicate that while both typical and atypical antipsychotic agents share the property of antagonizing the dopamine D2 receptor, the atypical antipsychotic agents are profoundly better at altering immune responses. Indeed, we have shown previously that risperidone and clozapine can inhibit the activation of LPS stimulated bone marrow derived macrophages but specific inhibitors of D1 or D2 does not, providing evidence that the immune modulating effects are acting directly on immune cells but not by D1 or D2 antagonist activity alone. Furthermore, we showed that administering sulpiride, an atypical antipsychotic which targets the D2 receptor specifically does not reduce EAE disease (D. O'Sullivan et al., 2014).

The immune modulating properties of atypical antipsychotic agents are likely more beneficial to patients with highly complex psychiatric and neurological disorders as not only dopamine antagonists, but also as neuroprotective and anti-inflammatory or immunomodulatory agents. 


\subsection{Neuroinflammation}

The CNS was once thought to be an immune privileged site due to the highly selective permeability of the blood-brain barrier, but it is now becoming a popular topic of research for its capacity to respond to inflammatory insults. Although immune responses in the brain can be beneficial to control infection and mediate repair of damaged tissue, inappropriate or excessive inflammatory responses can be unfavourable to the host. In fact, it is generally accepted that immune activation in the CNS contributes to the detrimental effects of many neurodegenerative diseases like stroke, schizophrenia and depression; all of which are associated with the activation of microglia (Chung, Welsh, Barres, \& Stevens, 2015; Perry, Nicoll, \& Holmes, 2010).

Of interest to the current topic of research is the debilitating psychiatric disorder schizophrenia. Historically believed to be an imbalance in neurotransmitter signalling, more recent evidence suggest that schizophrenia may not be due to a neurotransmitter imbalance alone or even at all. Several studies have shown, which has since been reviewed in a meta-analysis, that in re-lapsed schizophrenia patients' blood levels of inflammatory molecules IL-6, IL-8, TNF- $\alpha$, IFN- $\gamma$, TGF- $\beta$ and IL-1R $\alpha$ were significantly increased while the anti-inflammatory cytokine IL-10 was reduced when compared to healthy subjects (B. J. Miller, Buckley, Seabolt, Mellor, \& Kirkpatrick, 2011). Additionally, there is evidence to suggest that microglia are hyperactive in schizophrenic patients (Monji et al., 2013) and are further activated during an acute psychotic episode (Steiner et al., 2008). Of note, the cytokine IL-12, which is produced mainly by innate cells such as macrophages and microglia (Y. Sonobe et al., 2005; Xing, Zganiacz, \& Santosuosso, 2000), is abnormally high in schizophrenic patients and normalized after 8 weeks of treatment with atypical antipsychotic medication (Y. K. Kim et al., 2002). Indeed, when microglia are cultured in vitro and treated with atypical antipsychotic agents an alteration in activation towards a less inflammatory state is observed (Bian et al., 2008; X. Hu et al., 2012; T. Kato, A. Monji, S. Hashioka, \& S. Kanba, 2007).

Following these studies, more recent investigators propose that suppression of microglial responses by atypical antipsychotic agents may be an effective strategy for treating inflammation in schizophrenia (Monji et al., 2013), more experimental studies that probe into their immunomodulatory effect soon followed. Our laboratory has previously shown that the atypical antipsychotic agents risperidone and clozapine are able to effectively reduce disease severity in experimental autoimmune encephalomyelitis (EAE), an animal model for multiple 
sclerosis (MS) (D. O'Sullivan et al., 2014). This finding indicates that medicines such as the atypical antipsychotic agents that are effective in reducing neuroinflammation in schizophrenia may also be therapeutic in other diseases that share a common pathological mechanism like MS (Bogie et al., 2014).

\subsection{Aims and objectives}

This research project aims to characterize the mechanism of action by which atypical antipsychotic agents such as clozapine are able to reduce clinical disease in the experimental model of MS, EAE. This goal will be achieved through two independent aims.

1. To elucidate precisely the immunological and cellular mechanism by which atypical antipsychotic agents can reduce disease by focusing on $\mathrm{T}$ cells and macrophages

2. To identify specific cellular signaling events induced by atypical antipsychotic treatment that occur within $\mathrm{T}$ cells and macrophages and may mediate the immune-modulating effect. 


\section{Chapter 2: Materials and Methods}




\subsection{Mice}

C57BL/6J (CD45.2) mice were purchased from the Malaghan Institute of Medical Research (MIMR, Wellington, New Zealand) and then housed in the Victoria University of Wellington animal facility.

B6.SJL-Ptprc ${ }^{a}$ Pepc $^{b}$ BoyJ (CD45.1) breeding pairs were purchased from the MIMR and then bred in the Victoria University of Wellington animal facility.

Mice expressing a TCR with the Va3.2 and V $\beta 11$ TCR chain combination specific for $\mathrm{MOG}_{35-}$ ${ }_{55}$ peptide (2D2) were originally obtained from Professor Vijay Kuchroo (Bettelli et al., 2003) and bred at the Victoria University of Wellington animal facility. Maintenance of the $\mathrm{MOG}_{35}$ ${ }_{55}$ specific TCR was verified by flow cytometric analysis of V $\alpha 3.2$ and V $\beta 11$ TCR expression on splenic CD4 T cells.

All mice were housed in specific pathogen free conditions at the Victoria University of Wellington (VUW, Wellington, New Zealand) animal facility with a 12-hour light/dark cycle and regulated temperature. Handling of mice for experimental manipulation were performed inside a laminar flow hood using aseptic technique.

All experimental protocols are approved by the VUW Animal Ethics Committee under protocol $2014 \mathrm{R} 32$.

\subsection{Induction of experimental autoimmune encephalomyelitis}

\subsubsection{Induction of active EAE}

EAE was induced as previously described. Briefly, female C57BL/6J mice aged between 8-12 weeks were injected in the rear flanks with $50 \mu \mathrm{g} / \mathrm{mouse} \mathrm{MOG}_{35-55}$ (MEVGWYRSPFSRVVHLYRNGK; Genscript, Piscataway, NJ) peptide emulsified in incomplete Freund's adjuvant (Sigma-Aldrich, St. Louis, MO) containing $500 \mu \mathrm{g} / \mathrm{mouse} M$. tuberculosis (Difco, Lawrence, KS) subcutaneously (s.c). 200 ng/mouse Pertussis toxin (PTx; List Biochemical, Campbell, CA) diluted in PTx buffer (see Appendix) was injected intraperitoneally (i.p) on day of immunization (day 0) and two days post immunization (day 2). Mice are weighed and scored daily for disease using the following disease scale: 0 , normal; 1, half-tail paralysis; 2 , full tail paralysis; 3 , full paralysis of one hind limb; 4, full hind limb paralysis; 5, moribund (D. O'Sullivan et al., 2014). 


\subsubsection{Induction of adoptively transferred EAE}

To induce adoptively transferred EAE, donor C57BL/6J mice are injected in the rear flanks

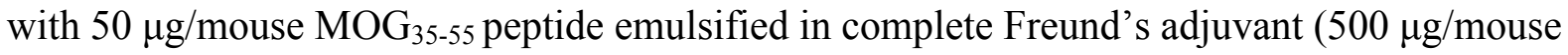
M. tuberculosis) subcutaneously as per active EAE induction. No pertussis injections are given to donor mice. Donor mice are euthanized 12 days post immunization and spleens and draining lymph nodes (mesenteric and inguinal; $\mathrm{dLN}$ ) are harvested and placed into $1 \mathrm{~mL}$ of wash buffer in a $1.7 \mathrm{~mL}$ centrifuge tube. dLN's are processed into single cell suspensions by forced passage through a $70 \mu \mathrm{M}$ cell strainer (BD Biosciences, Franklin Lakes, NJ). $10 \mathrm{~mL}$ of wash buffer is added through the strainer to wash remaining cells and then centrifuged at $760 \mathrm{x} g$ for 5 minutes. Supernatant is then removed and $2 \mathrm{~mL}$ of Red Blood Cell Lysis Buffer (SigmaAldrich, MO) is added and incubated at RT for 2 minutes to lyse red blood cells, after which 8 $\mathrm{mL}$ of wash buffer is added. Lysed samples are then centrifuged again at $760 \mathrm{xg}$ and resuspended in $10 \mathrm{~mL}$ of complete $\mathrm{T}$ cell medium (CTCM; see appendix III) and then live cell concentration was counted by trypan blue exclusion using a haemocytometer as described. Cells are then cultured at $5 \times 10^{6}$ cells $/ \mathrm{mL}$ in media supplemented with $50 \mu \mathrm{g} / \mathrm{mL} \mathrm{MOG}_{35-55,20}$ ng/mL IL-12p70 (Peprotech, NJ) and $10 \mu \mathrm{g} / \mathrm{mL}$ anti-IFN- $\gamma$ (clone XMG1.2; BioXcell, West Lebanon, $\mathrm{NH}$ ) for 72 hours in T25 tissue culture flasks (BD Biosciences, NJ) to allow $\mathrm{MOG}_{35}$ 55 specific T cells to expand. Non-adherent cells were collected, washed and re suspended in dPBS. $20 \times 10^{6}$ cells/mouse in $200 \mu \mathrm{L}$ of dPBS was injected i.p into naive recipient C57BL/6J or congenic B6-SJ ${ }^{\text {ptprca }}$ mice. All recipient mice received $200 \mathrm{ng} /$ mouse pertussis toxin i.p 4 hours post transfer of cells and 2 days post transfer. Mice are weighed and scored regularly for disease presentation and scored using the same scoring method described in active EAE.

\subsection{In vivo techniques}

\subsubsection{Oral drug administration}

\section{Oral administration by feed}

Olanzapine tablets were crushed up using a mortar and pestle to create a fine powder. Animal feed was powdered by passing dry pellets through a meat mincer. Olanzapine or clozapine were then added to powdered feed at a concentration that is calculated to achieve daily dose based on an average feed consumption of $4 \mathrm{~g} /$ mouse (Bachmanov, Reed, Beauchamp, \& Tordoff, 2002). 


$$
D D=\frac{S D \times B W}{F I}
$$

$\mathbf{D D}=\operatorname{diet} \operatorname{dose}(\mathrm{mg} / \mathrm{kg})$

SD $=$ daily dose $(\mathrm{mg} / \mathrm{kg})$

BW $=$ body weight $(\mathrm{g})$

$\mathbf{F I}=$ food intake $(4 \mathrm{~g} /$ day $)$

\subsubsection{Oral administration by drinking water}

Clozapine and risperidone (kindly provided by Douglas Pharmaceuticals, Auckland, NZ) were dissolved in $0.1 \mathrm{M}$ acetic acid (Sigma-Aldrich, MO) at a concentration of $6 \mathrm{mg} / \mathrm{mL}$. Fluoxetine (Actavis, Parsippany-Troy Hills, $\mathrm{NJ}$ ) was dissolved is $\mathrm{dH}_{2} \mathrm{O}$ at a concentration of $6 \mathrm{mg} / \mathrm{mL}$ overnight and non-soluble binding material was removed by centrifugation. Stock concentrations were then diluted in autoclaved tap water to a final concentration that is calculated to achieve the daily dosage based on average daily water consumption of 4 $\mathrm{mL} / \mathrm{mouse} /$ day.

Sinemet ${ }^{\circledR ~ 100 / 25 ~(M e r c k ~ \& ~ C o ., ~ K e n i l w o r t h, ~ N J) ~ t a b l e t s ~ w e r e ~ c r u s h e d ~ u p ~ u s i n g ~ a ~ m o r t a r ~ a n d ~}$ pestle and dissolved in $\mathrm{dH}_{2} \mathrm{O}$ overnight at a concentration of $2 \mathrm{mg} / \mathrm{mL}$ of L-DOPA. Non-soluble binding material was centrifuged and removed. Stock concentration was then diluted to the final concentration in tap water to achieve the correct daily dose based on an average daily water consumption of $4 \mathrm{~mL} / \mathrm{mouse} /$ day.

\subsubsection{In vivo proliferation assay}

In vivo proliferation assay was performed as previously described (David O'Sullivan, Miller, Northcote, \& La Flamme, 2013). Briefly, spleens, mesenteric and inguinal lymph nodes were isolated from donor 2D2 mice (CD45.2) and single cell suspensions were made by forced passage through a $70 \mu \mathrm{M}$ cell strainer (BD Biosciences, NJ). Red blood cells were lysed using Red Blood Cell Lysis Buffer (Sigma-Aldrich, MO) and washed with Dulbecco's phosphate buffered saline (dPBS; Thermo Fisher Scientific, Waltham, MA). Cell suspensions were stained with CFSE ( $5 \mu \mathrm{M}$; Thermo Fisher Scientific, MA) as per manufacturers recommendation. Cells were washed three times by centrifuging at $760 \mathrm{x} g$ for 5 minutes and re-suspended in dPBS. 20x $10^{6}$ cells/mouse was injected i.p into recipient congenic B6-SJ ${ }^{\text {ptprca }}$ 
(CD45.1) one-day prior to immunization. Recipient mice were then immunized for EAE as described. At euthanasia; blood, draining lymph nodes and spleens were isolated and processed for flow cytometric analysis of CFSE dilution using a FACS Canto II flow cytometer (BD Biosciences, NJ). Proliferation index (total number of divisions divided by the number of cells that went into division) and percent proliferated were calculated using FlowJo proliferation platform (FlowJo LLC, Ashland, OR).

\subsubsection{Regulatory $T$ cell neutralization}

Female C57BL/6J mice were injected with $200 \mu \mathrm{g} /$ mouse rat anti-mouse CD25 (clone PC61; BioXCell, NH) to neutralize Treg (Setiady, Coccia, \& Park, 2010) or Rat IgG (Sigma-Aldrich, MO) as a control i.p, 3 days before the induction of EAE. $200 \mu \mathrm{g} /$ mouse of antibody was used as it was sufficient for complete binding of CD25. Subsequent injections were performed at 7 and 14 days post immunization to maintain neutralization. EAE was induced and scored as previously described.

\subsection{Ex vivo techniques}

\subsubsection{Trypan blue exclusion}

For counting live cells, single cell suspensions were re-suspended in wash buffer or CTCM. Samples were diluted 1:10 by adding $10 \mu \mathrm{L}$ of sample into a U-bottomed 96 well plate followed by $90 \mu \mathrm{L}$ of trypan blue solution (Thermo Fisher Scientific, MA) and mixed thoroughly by gently pipetting. $10 \mu \mathrm{L}$ of this dilution was then placed under the glass cover slip placed on a Neubauer haemocytometer (Hawksley, Lancing, UK) and counted using a compound microscope (CX41: Olympus, PA, USA).

\subsubsection{Processing and isolation of spleen into single cell suspensions}

Mice were euthanized by $\mathrm{CO}_{2}$ asphyxiation and covered in $100 \%$ ethanol for aseptic handing from herein. All surgical tools were sterilized in $100 \%$ ethanol before and during use. Spleens were removed and placed in $1 \mathrm{~mL}$ sterile wash buffer (see Appendix III) on ice. A single cell suspension was prepared by forced passage of the spleen through a $70 \mu \mathrm{M}$ cell strainer (BD Biosciences, NJ) placed on top of a sterile $50 \mathrm{~mL}$ conical tube (Corning, Corning, NY) using a sterile syringe plunger from a $1 \mathrm{~mL}$ tuberculin syringe (BD Biosciences, $\mathrm{NJ}$ ). Remaining 
cells in the cell strainer was then washed into the conical tube using $10 \mathrm{~mL}$ of wash buffer. Splenocytes were centrifuged at $760 \mathrm{x}$ g for 5 minutes to wash. Supernatant was then removed and $2 \mathrm{~mL}$ of Red Blood Cell Lysis Buffer (Sigma-Aldrich, MO) was added for 2 minutes to lyse red blood cells. Lysis was quenched by adding $8 \mathrm{~mL}$ of wash buffer and centrifuging at $760 \mathrm{x}$ g for $5 \mathrm{mins}$ before re-suspending the splenocytes in $10 \mathrm{~mL}$ of wash buffer. Live cells were counted by trypan blue exclusion as described and then re-suspended to the desired cell density with CTCM for cell culture or FACS buffer (see Appendix III) for flow cytometric analysis.

\subsubsection{Processing and isolation of lymph nodes into single cell suspensions}

Mice were euthanized by $\mathrm{CO}_{2}$ asphyxiation and covered in $100 \%$ ethanol for aseptic handing from herein. All surgical tools were sterilized in 100\% ethanol before and during use. Mesenteric and inguinal lymph nodes were carefully removed and placed in $1 \mathrm{~mL}$ sterile wash buffer (see Appendix III) on ice. A single cell suspension was prepared by forced passage of the pooled lymph nodes through a $70 \mu \mathrm{M}$ cell strainer (BD Biosciences, NJ) placed on top of a sterile $50 \mathrm{~mL}$ conical tube (Corning, NY) using a sterile syringe plunger from a $1 \mathrm{~mL}$ tuberculin syringe (BD Biosciences, NJ). Remaining cells in the cell strainer was then washed into the conical tube using $10 \mathrm{~mL}$ of wash buffer. Lymph node cells were centrifuged at $760 \mathrm{x}$ $\mathrm{g}$ for 5 minutes to wash. Supernatant was then removed and $2 \mathrm{~mL}$ of Red Blood Cell Lysis Buffer (Sigma-Aldrich, MO) was added for 2 minutes to lyse red blood cells. Lysis was quenched by adding $8 \mathrm{~mL}$ of wash buffer and centrifuging at $760 \mathrm{xg}$ for $5 \mathrm{mins}$ before resuspending the lymph node cells in $10 \mathrm{~mL}$ of wash buffer. Live cells were counted by trypan blue exclusion as described and then re-suspended to the desired cell density with CTCM) for cell culture or FACS buffer (see Appendix III) for flow cytometric analysis.

\subsubsection{Processing and isolation of brain into single cell suspension}

Mice were euthanized by $\mathrm{CO}_{2}$ asphyxiation and covered in $100 \%$ ethanol for aseptic handling from herein. All surgical tools were sterilized in 100\% ethanol before and during use. The peritoneal cavity was opened with scissors and the liver portal vein severed. $20 \mathrm{~mL}$ of sterile PBS was then perfused through the left ventricle of the heart slowly to ensure complete perfusion using a $20 \mathrm{~mL}$ syringe (BD Biosciences, $\mathrm{NJ}$ ) and a $23 \mathrm{G}$ needle (BD Biosciences, NJ). After perfusion, mice were decapitated with scissors. Brains were removed with small tweezers by first removing the top region of the skull and then carefully lifting the brain to 
maintain integrity and structure. Brains were fixed in 4\% paraformaldehyde (PFA) in a $15 \mathrm{~mL}$ conical tube.

\subsubsection{Processing and isolation of spinal cord into single cell suspension}

Mouse brains were first removed as described above. Firstly, the spinal cord was removed using a $10 \mathrm{~mL}$ syringe of PBS equipped with a 19G needle inserted into the spinal column. The spinal cord removed by hydraulic extrusion by forcing PBS through the spinal column. The spinal cord was then placed into $1 \mathrm{~mL}$ PBS in a $1.7 \mathrm{~mL}$ micro centrifuge tube and placed on ice. Spinal cords were diced into smaller pieced using a surgical blade and digested with $5 \mathrm{~mL}$ collagenase type II (2.4 mg/mL in PBS; Thermo Fisher Scientific, MA) for 30 minutes at $37^{\circ} \mathrm{c}$. Spinal cords were then further homogenized by repeat pipetting through a $1 \mathrm{~mL}$ single channel pipette. Spinal cords were further digested for 10 minutes at $37^{\circ} \mathrm{c}$ and then filtered through a $70 \mu \mathrm{M}$ cell strainer. Collagenase was removed by centrifuging at $760 \mathrm{x}$ g for 5 minutes and then re-suspended into $10 \mathrm{~mL}$ of 37\% Percoll (see Appendix III) in a $15 \mathrm{~mL}$ conical tube and centrifuged for 30 minutes at $760 \mathrm{x} \mathrm{g}$ with the brakes turned off. After separation the myelin layer and Percoll were carefully removed and the cell pellet re-suspended in $1 \mathrm{~mL}$ of FACS buffer for flow cytometric analyses.

\subsubsection{Blood collection for flow cytometry}

Firstly, $1 \mathrm{~mL}$ insulin syringes (BD Biosciences, NJ) were coated with EDTA by collecting 0.5 M EDTA into the syringe and expelling completely. $20 \mu \mathrm{L} 0.5 \mathrm{M}$ EDTA was then added to each $1.7 \mathrm{~mL}$ micro centrifuge tube. Approximately $200 \mu \mathrm{L}$ of blood was then added to each tube, mixed immediately and placed on ice. Whole blood was then transferred into a $15 \mathrm{~mL}$ conical flash and a red blood cell lysis performed as described and was repeated until red blood cells were sufficiently lysed. Each blood sample was then washed and re-suspended in FACS buffer on ice for flow cytometric analysis.

\subsection{Cytokine assays}

\subsubsection{General ELISA protocol}

Enzyme linked immunosorbent assays (ELISA) were performed by coating 96 well Nunc MaxiSorp plates (Thermo Fisher Scientific, MA) with a primary capture antibody in ELISA 
capture buffer (see Appendix III) and incubated overnight at $4^{\circ} \mathrm{c}$. Capture antibody solution was removed and plates were washed in wash buffer containing PBS with $0.01 \%$ Tween-20 (Sigma-Aldrich, MO) 4 times. Plates were blocked with 10\% foetal calf serum (FCS) in PBS for 2 hours at room temperature (RT). $50 \mu \mathrm{L} /$ well of cytokine standards and samples were then added to each well and incubated for 2 hours RT or overnight at $4^{\circ} \mathrm{c}$. The standard curve was prepared by serially diluting in the top two rows of the plate. Plates were then washed 4 times in wash buffer and $50 \mu \mathrm{L} /$ well of the matched biotinylated secondary antibody was added and incubated RT. After 1 hour plates were washed 6 times in wash buffer and $50 \mu \mathrm{L} /$ well of streptavidin-horseradish peroxidase (SA-HRP) was added and incubated for a further 1-hour RT. After incubation, plates are washed 8 times and tetramethyl benzidine (TMB) substrates $\mathrm{A}$ and $\mathrm{B}$ (BD Biosciences, $\mathrm{NJ}$ ) were brought to room temperature and mixed to equal volumes. $100 \mu \mathrm{L} /$ well of TMB reagent was added and the reaction was stopped with $100 \mu \mathrm{L} /$ well $0.18 \mathrm{M}$ $\mathrm{H}_{2} \mathrm{SO}_{4}$. Details for specific ELISA's including capture buffer used, antibody and SA-HRP concentrations can be found in Appendix $\mathbf{V}$.

\subsubsection{Cytokine Bead Assay}

Cytokine bead assay was performed using a LEGENDplex Mouse inflammation kit (Biolegend, San Diego, CA) as per the manufacturers recommendations. Briefly, an 8 point standard curve was prepared by serial dilution of cytokine standards included in the kit. $25 \mu \mathrm{L}$ of assay buffer was added to each well of a 96 well V-bottom plate (Corning, NY) followed by $25 \mu \mathrm{L}$ of standards and samples. $25 \mu \mathrm{L}$ of the mixed beads were then added to each sample/standard well and wrapped in foil, incubated for 2 hours at room temperature in the dark on an orbital plate shaker. After incubation, the plate was centrifuged at $250 \mathrm{xg}$ for $5 \mathrm{mins}$ to pellet beads. Supernatant was then flicked out and the plate blotted on a paper towel to absorb excess liquid. Beads re-suspended by gently vortexing. $200 \mu \mathrm{L}$ of washing buffer was then added to each well and the plate was centrifuged again at $250 \mathrm{x}$ g for 5 minutes to pellet beads. Supernatant was again removed followed by adding $25 \mu \mathrm{L}$ of detection antibody to each well. Detection antibody was incubated at room temperature for 1 hour on an orbital plate shaker. After incubation $25 \mu \mathrm{L}$ of streptavidin-phycoerythrin was added to each well and incubated for 30 minutes at room temperature on an orbital plate shaker. Plates were then washed once again before resuspending beads in $150 \mu \mathrm{L}$ of wash buffer. Samples were analysed on a FACS Canto II flow cytometer (BD Biosciences, NJ) set up according to the manufacturers recommendations. 


\subsection{Cell culture and in vitro experiments}

\subsubsection{Compounds for in vitro use}

Dopamine hydrochloride (Sigma-Aldrich, MO) was dissolved in $\mathrm{dH}_{2} \mathrm{O}$ at a stock concentration of $25 \mathrm{mM}$ and stored at $-80^{\circ} \mathrm{c}$ until required.

Clozapine (Kindly provided by Douglas Pharmaceuticals, Auckland, New Zealand) was dissolved in $0.1 \mathrm{M}$ acetic acid (Sigma-Aldrich, MO) at a concentration of $25 \mathrm{mM}$ and stored at $-80^{\circ} \mathrm{c}$ until required.

CHIR99021 (Sigma-Aldrich, MO) was dissolved in DMSO at a stock concentration of 100 $\mathrm{mM}$ and stored at $-80^{\circ} \mathrm{c}$ until required.

Akt1/2 kinase inhibitor (Sigma-Aldrich, MO) was dissolved in DMSO at a stock concentration of $100 \mathrm{mM}$ and stored at $-80^{\circ} \mathrm{c}$ until required.

Forskolin (Sigma-Aldrich, MO) was dissolved in absolute ethanol at a concentration of $10 \mathrm{mM}$ and stored at $-20^{\circ} \mathrm{c}$ until required.

All stock compounds were stored in single use aliquots to prevent repeated freeze/thaw cycles. Compounds were diluted to the desired concentration in CTCM for in vitro assays.

\subsubsection{Culture and maintenance of RAW 264.7 macrophage cell line}

RAW 264.7 cells were a kind gift from the Malaghan Institute of Medical Research. $2 \times 10^{6}$ RAW264.7 cells were stored long term by re-suspending cells in cryopreservation medium (see Appendix III) and kept in a liquid phase liquid nitrogen storage tank until required. Low passage numbers $(<20)$ RAW264.7 cells were cultured from storage by first thawing the cryovial in a $37^{\circ} \mathrm{c}$ water bath, after which its contents were immediately transferred into $9 \mathrm{~mL}$ of $37^{\circ} \mathrm{c} \mathrm{CTCM}$ and centrifuged at $300 \mathrm{x} \mathrm{g}$ for 5 minutes to remove the cryopreservation medium. The cell pellet was then re-suspended in $10 \mathrm{~mL}$ of $37^{\circ} \mathrm{c} \mathrm{CTCM}$ and transferred to a T25 tissue culture flask and incubated in a humidified incubator at $37^{\circ} \mathrm{c}$ and $5 \% \mathrm{CO}_{2}$ until confluent. At $80 \%$ confluency, RAW264.7 cells were passaged by first removing culture medium and adding $5 \mathrm{~mL}$ of warm dPBS to wash non-adherent cells/debris to be discarded. 5 $\mathrm{mL}$ of cold $\mathrm{dPBS}$ is then added to the flask and adherent cells were lifted from the flask by using a cell scraper. The cell suspension is then aspirated and transferred to a $15 \mathrm{~mL}$ conical 
tube and centrifuged at $300 \mathrm{x}$ g for $5 \mathrm{mins}$ and re-suspended in $1 \mathrm{~mL}$ of dPBS. Live cells are counted by trypan blue exclusion. $1 \times 10^{6}$ cells are added to $15 \mathrm{~mL}$ of CTCM in a T75 culture flask and cultured until $80 \%$ confluent.

\subsubsection{Isolation and culture of bone marrow derived macrophages}

Bone-marrow derived macrophages were isolated and cultured as described (Tierney et al., 2008). Briefly, female C57BL/6J mice aged between 8-12 weeks were euthanized by $\mathrm{CO}_{2}$ asphyxiation. Bone marrow cells were isolated from the femurs and tibias by flushing the bone marrow from each bone with $5 \mathrm{~mL}$ of dPBS containing 1\% PenStrep (Thermo Fisher Scientific, MA) into a sterile $50 \mathrm{~mL}$ conical tube. Bone marrow is homogenized by repeatedly pipetting through a $5 \mathrm{~mL}$ serological pipette. RBC's were lysed as previously described and bone marrow cells cultured overnight in CTCM at $1 \times 10^{6}$ cells $/ \mathrm{mL}$ in tissue culture flasks. Nonadherent hematopoietic progenitor cells were then isolated and cultured in sterile $90 \mathrm{~mm}$ petri dishes in the presence of IL-3 (5 ng/mL; Peprotech, Rocky Hill, NJ) and granulocytemacrophage colony-stimulating factor (GM-CSF; $5 \mathrm{ng} / \mathrm{mL}$; Peprotech, NJ) at a volume of 5 $\mathrm{mL}$. After 4 days of culture, $5 \mathrm{~mL}$ of CTCM containing IL-3 $(5 \mathrm{ng} / \mathrm{mL})$ and GM-CSF $(5 \mathrm{ng} / \mathrm{mL}$; Peprotech, NJ) is added to each culture dish. After another 4 days, bone-marrow derived macrophages were harvested by first washing non-adherent cells with warm dPBS and then repeatedly blasting with ice cold dPBS to detach macrophages. Macrophages were plated into flat bottom 96 well plates at 100,000 cells/well and primed with IFN- $\gamma$ for up to 18 hours. Macrophages were then stimulated with $200 \mathrm{ng} / \mathrm{mL}$ lipopolysaccharide (LPS; Sigma-Aldrich, MO) and indicated compounds for 24 hours. At the end of the experiment, supernatant was removed and used or stored at $-20^{\circ} \mathrm{c}$ immediately. Cell viability assay was performed by MTT assay as described.

\subsubsection{T cell differentiation assay}

Spleens were isolated from female 2D2 $\mathrm{TCR}^{\mathrm{MOG} 35-55}$ mice aged between 8-12 weeks of age and a single cell suspension was prepared as previously described. Splenocytes were then cultured in CTCM at $1 \times 10^{6}$ cells/well in a flat bottomed 96 well plate (Corning, NY) and stimulated with $\mathrm{MOG}_{35-55}$-peptide $(50 \mu \mathrm{g} / \mathrm{mL})$ in media alone or medium containing rIL-12p70 (20 ng/mL; Peprotech, NJ) and anti-IL-4 (11B11, a kind gift from the Malaghan Institute; 10 $\mu \mathrm{g} / \mathrm{mL}$,) for Th1; rIL-6 (20 ng/mL; BD Biosciences, NJ), rTGF-ß1 (5 ng/mL; Thermo Fisher Scientific, MA), anti-IFN- $\gamma(10 \mu \mathrm{g} / \mathrm{mL}$; BioXcell, NH) for Th17 and rTGF-ß1 $(5 \mathrm{ng} / \mathrm{mL}$; 
Thermo Fisher Scientific, MA), anti-IFN- $\gamma(10 \mu \mathrm{g} / \mathrm{mL}$; BioXcell, NH), anti-IL-6 (10 $\mu \mathrm{g} / \mathrm{mL}$; MP5-20F3; BD Biosciences, NJ) for Treg, for 72 hours. T cell differentiation was then assessed by intracellular cytokine/antigen staining as described. Plates were centrifuged at $400 \mathrm{xg}$ for 5 minutes and supernatants were collected and stored at $-20^{\circ} \mathrm{c}$ for cytokine analysis at a later time.

\subsubsection{MTT reduction assay}

Following completion of an experiment, $170 \mu \mathrm{L} /$ well of supernatant was transferred to a sterile $\mathrm{U}$ bottom 96 well plate and stored at $-20^{\circ} \mathrm{c}$ or used immediately. $50 \mu \mathrm{L} /$ well of fresh CTCM warmed to $37^{\circ} \mathrm{c}$ was added before adding $20 \mu \mathrm{L} /$ well of MTT solution (see Appendix III) was added to each well. Plates were incubated for a further 2 hours at $37^{\circ} \mathrm{c}$ and $5 \% \mathrm{CO}_{2}$. After incubation, the reaction was stopped by adding $100 \mu \mathrm{L} /$ well of MTT stop solution (see Appendix III) and left overnight at $37^{\circ} \mathrm{c}$ to solubilize formazan products. Absorbance was measured at $550 \mathrm{~nm}$ in a multi-well Enspire Multilabel plate reader (PerkinElmer, Wellesley, MA). Cell metabolism was calculated as percentage absorbance of indicated control wells.

\subsubsection{Measuring intracellular cyclic-AMP}

Bone-marrow derived macrophages were seeded onto 96 well plates at 100,000 cells/well and primed for 18 hours with $20 \mathrm{U} / \mathrm{mL}$ of rIFN- $\gamma$. Supernatants were removed and replaced with $100 \mu \mathrm{L}$ CTCM containing 0.5 mM 3-Isobutyl-1-methylxanthine (IBMX; Sigma-Aldrich, MO) for 15 minutes before adding indicated compounds and stimulating with $200 \mathrm{ng} / \mathrm{mL}$ of lipopolysaccharide (LPS; Sigma-Aldrich, MO) for 15, 30, 45 and 60 minutes. All compounds and LPS were diluted in CTCM containing $0.5 \mathrm{mM}$ IBMX to maintain overall IBMX concentration. After stimulation, supernatants were removed swiftly by flicking and each well was washed with $200 \mu \mathrm{L}$ of warm dPBS twice by flicking out the supernatant and repeating. After washing, $50 \mu \mathrm{L}$ of ice-cold absolute ethanol was added to each well to fix cells and placed in a $37^{\circ} \mathrm{c}$ dry incubator until all ethanol has completely evaporated. $50 \mu \mathrm{L}$ of lysis buffer was then added to each well and the plate was placed on an orbital plate shaker for 30 minutes at $4^{\circ} \mathrm{c}$. Cell lysates were then used immediately or stored at $-80^{\circ} \mathrm{c}$ for later analysis.

Intracellular cAMP from cell lysates were measured using the AlphaScreen cAMP detection kit (Perkin Elmer, MA) according to the manufacturers protocol. Briefly, a 12-point cAMP standard curve was prepared by serial dilution of the supplied cAMP standard. Cell lysates and 
standards were added to an opaque white 384 well plate (Corning, NY) at $10 \mu \mathrm{L} /$ well. Acceptor buffer was then added to each well at $5 \mu \mathrm{L} /$ well followed by $15 \mu \mathrm{L} /$ well of donor buffer. The plate was then sealed and incubated for 8-12 hours before reading the plate on a M1000 Pro plate reader (Tecan, Mannedorf, Switzerland) using AlphaScreen settings. Data was then graphed and analysed using GraphPad Prism 6 (GraphPad Software, CA, USA).

\subsubsection{Measuring phosphorylation of cell signaling proteins}

Bone-marrow derived macrophages were seeded onto 96 well plates at 100,000 cells/well and primed for 18 hours with $20 \mathrm{U} / \mathrm{mL}$ of rIFN- $\gamma$. Compounds were then added at indicated concentrations and stimulated with $200 \mathrm{ng} / \mathrm{mL}$ of LPS for the indicated time. After stimulation, supernatant was removed and each well was washed with $200 \mu \mathrm{L}$ of warm dPBS twice before adding $50 \mu \mathrm{L}$ of Milliplex lysis buffer containing 1\% protease inhibitor cocktail (SigmaAldrich, MO). Cells were lysed on an orbital shaker at $4^{\circ} \mathrm{c}$ for $30 \mathrm{mins}$ and assayed immediately using the MILLIPLEX MAP Multi-Pathway Magnetic Bead 9-Plex Total protein and phosphorylated protein kits (Merck Millipore, Billerica, MA) with additional MAP Mate GSK$3 \beta$ Total and phosphorylated protein kits (Merck Millipore, MA) according to the manufacturers protocol. Plates were read on a Bio-Plex 100 instrument (Bio-Rad Laboratories, Hercules, CA).

\subsubsection{Splenocyte MOG $_{35-55}$-peptide re-stimulation assay}

Spleens were processed into single cell suspension as described. Splenocytes were resuspended into warm CTCM at $10 \times 10^{6}$ cells $/ \mathrm{mL}$ and seeded onto flat bottom 96 well plates (BD Biosciences, CA) at $1 \times 10^{6}$ cells/well. MOG $_{35-55}$-peptide was added to each well and incubated for 72 hours at $37^{\circ} \mathrm{c}$ and $5 \% \mathrm{CO}_{2}$. After stimulation, plates were centrifuged at $400 \mathrm{x}$ g for 5 minutes and the supernatant was transferred to a U bottomed 96 well plate (BD Biosciences, CA) and stored at $-20^{\circ} \mathrm{c}$ or used immediately for cytokine assays.

\subsection{Flow cytometry}

\subsubsection{Cell surface marker staining}

Cells were harvested and suspended in FACS buffer at $10^{6}$ cells/well in a U bottom 96 well plate. Fc receptors were blocked using $50 \mu \mathrm{L} /$ well anti CD16/CD32 (clone 2.4G2) (BD 
Biosciences, NJ) and incubated on ice for 15 minutes. $200 \mu \mathrm{L} /$ well of FACS buffer was then added and plates were centrifuged at $400 \mathrm{x} g$ for 5 minutes and the supernatant flicked out. Plates were vortexed to re-suspend cells and $50 \mu \mathrm{L} /$ well of primary antibody is added and left on ice, protected from light for 30 minutes. Cells were then washed twice with $200 \mu \mathrm{L}$ FACS buffer and finally re-suspended in $300 \mu \mathrm{L}$ of FACS buffer in a U bottomed 96 well plate or 5 $\mathrm{mL}$ polystyrene tubes (BD Biosciences, NJ) and analysed on a FACS Canto II (BD Biosciences, NJ) flow cytometer. Data was analysed using FlowJo Software (FlowJo LLC, Ashland, OR).

\subsubsection{Intracellular antigen staining}

To stain for intracellular antigens, cells are prepared and stained for extracellular markers as described but are fixed with $120 \mu \mathrm{L} /$ well of Fix/Perm buffer (BD Biosciences, NJ) after the final washing step and left for 50 minutes on ice and protected from light. $100 \mu \mathrm{L} /$ well of Perm/Wash Buffer (BD Biosciences, NJ) is added and centrifuged at $650 \mathrm{x} \mathrm{g}$ at $4^{\circ} \mathrm{c}$. Cells are washed once more in $200 \mu \mathrm{L} /$ well of Perm Buffer and then $50 \mu \mathrm{L} /$ well of antibody in Perm/Wash Buffer is added and left to stain for 1 hour on ice and protected from light. After staining, cells are washed twice in $200 \mu \mathrm{L} /$ well of Perm/Wash Buffer before washing once and resuspending in $300 \mu \mathrm{L}$ of FACS buffer in a U bottomed 96 well plate or $5 \mathrm{~mL}$ polystyrene tubes (BD Biosciences, NJ) and analysed on a FACS Canto II (BD Biosciences, NJ). Antibodies used can be found in Appendix IV.

\subsubsection{Intracellular cytokine staining}

For intracellular cytokine staining, cells were first stimulated with Phorbol 12-myristate 13acetate (PMA; 50 ng/mL; Sigma-Aldrich, MO) and Ionomycin (500 ng/mL; Sigma-Aldrich, MO) in the presence GolgiStop/Monensin (BD Biosciences, NJ) for 5 hours at $37^{\circ} \mathrm{c}$ and $5 \%$ $\mathrm{CO}_{2}$. Protocol for staining intracellular cytokines was then performed in the same way as the intracellular antigen staining protocol described above. Samples were then analysed on a FACS Canto II (BD Biosciences, NJ). Antibodies used can be found in Appendix IV.

\subsection{Statistical Analyses}

All graphs were prepared using GraphPad Prism 6 (GraphPad Software, CA). All statistical analyses were conducted using GraphPad Prism 6. Student's t-test was performed when 
comparing two groups that were normally distributed. When comparing two groups that were not normally distributed, a Mann-Whitney test was performed instead. Statistical analyses for experiments with multiple groups was performed by a one-way ANOVA and Dunnett's multiple comparison test. When comparing multiple groups with more than one categorical variable, a two-way ANOVA was performed followed by Sidak's multiple comparison test. P values of less than 0.05 were considered statistically significant. 
Chapter 3: Using clozapine to treat experimental autoimmune encephalomyelitis 


\subsection{Introduction}

Inflammatory diseases of the central nervous system such as multiple sclerosis cause permanent damage to nervous tissue leading to impaired cognitive function. In multiple sclerosis, this can lead to loss of vision, imbalance, depression, pain and multiple motor disorders (Compston \& Coles, 2008). Multiple sclerosis is the most common inflammatory disease of the central nervous system and is often treated clinically with immune modulating agents that target activation, proliferation or the trafficking of immune cells to the CNS (Dendrou et al., 2015). There are now a number of approved treatment options such as glatiramer acetate, interferon beta and natalizumab; however, they are not effective in all cases and are associated with adverse effects and increased risk of opportunistic infections (Winkelmann, Loebermann, Reisinger, Hartung, \& Zettl, 2016). Unfortunately, once disease develops to the progressive stages, there are no available treatments (English \& Aloi, 2015) and the lack of treatment options for these forms of disease remains a significant public health concern as patients continually accumulate disabilities.

Demyelination, neurodegeneration and the formation of sclerotic plaques is generally associated with the chronic neuroinflammatory process that leads to accumulating disability (Dendrou et al., 2015). One of the most important drivers of this pathological process is the inflammatory activation of microglia and macrophages within the CNS, and these have been shown in MS lesions to display a classical type of activation (D. Y. Vogel et al., 2013) and remain chronically active during disease (Fischer et al., 2012). Whereas alternatively-activated macrophages and microglia have anti-inflammatory and wound repair properties, classicallyactivated macrophages and microglia can be detrimental to tissue and have been implicated in many diseases of the CNS (Cherry, Olschowka, \& O'Banion, 2014). In particular, this pathological feature of MS shares striking similarities with the inflammatory processes that drive chronic inflammation and neurodegenerative damage observed during schizophrenia (Laskaris et al., 2016; Monji, Kato, \& Kanba, 2009), which was reviewed recently (Müller, Weidinger, Leitner, \& Schwarz, 2015). Recent studies suggest that anti-inflammatory medications may be beneficial in treating the symptoms of schizophrenia (Monji et al., 2009; Müller et al., 2015), and the notion that atypical antipsychotic agents such as clozapine and risperidone may reduce the symptoms of schizophrenia by their anti-inflammatory and neuroprotective properties suggest that they may be beneficial for other inflammatory diseases of the CNS, including MS. Moreover, given the vital role of microglia and macrophages in the 
pathology of MS, investigators suggest that therapeutic targeting of myeloid cells could provide great improvements in the therapeutic management of MS (M. K. Mishra \& V. W. Yong, 2016). Indeed, we have shown that clozapine and risperidone are able to effectively reduce disease during EAE (D. O'Sullivan et al., 2014), an animal model for MS. This chapter aims to investigate the effects of clozapine on disease parameters using different treatment regimens to assess its potential as a therapeutic agent for multiple sclerosis and develop a model in which to investigate the precise mechanism by which clozapine is able to reduce EAE disease.

\subsection{Aim}

The aim of this chapter is to study and optimize the effect of treatment with atypical antipsychotic agents on disease and immunological parameters during EAE.

\subsubsection{Specific Aims}

1. To study disease progression during active EAE induction with prophylactic clozapine treatment

2. To whether disease protection by clozapine is associated with changes in peripheral immune cell subsets

3. To evaluate the effect of clozapine when given therapeutically to assess its feasibility as a MS therapeutic 


\subsection{Results}

\subsubsection{Clozapine treatment attenuates the disease course of EAE}

Consistent with our previously published findings, mice treated with $60 \mathrm{mg} / \mathrm{kg} / \mathrm{day}$ of clozapine experience an attenuated EAE disease course (D. O'Sullivan et al., 2014). Clozapine treated mice experienced attenuated and delayed disease compared to vehicle treated mice (Figure 3.1A). Weight loss is apparent in clozapine treated EAE mice when compared to vehicle treated EAE mice after the initial addition of clozapine to drinking water but weight stabilizes by 7 d.p.i and is maintained thereafter (Figure 3.1B). Interestingly, clozapine treatment did not induce significant weight loss in unimmunized healthy controls. As disease progresses to 11 d.p.i, vehicle treated mice experience weight loss that becomes significantly greater than that of clozapine treated mice by 13 d.p.i (Figure 3.1B) whilst clozapine treated mice are pre-clinical.

Untreated mice reached a mean peak disease score of 2.9 whereas clozapine treated mice reached only a mean score of 0.4 (Figure 3.1C). In addition, clozapine significantly delayed the onset of EAE disease in mice that got sick from 11.8 d.p.i in vehicle treated mice to 14.5 d.p.i in clozapine treated mice (Figure 3.1D). Overall disease burden was reduced from 7.7 in vehicle treated mice to 0.5 in clozapine treated mice (Figure 3.1E). It is important to note that these experiments were ended at 15 d.p.i. Daily water consumption was measured to monitor drug intake and although EAE did not have a significant effect on water consumption, adding clozapine to the water reduced water consumption of mice significantly when compared to vehicle (Figure 3.1F). 

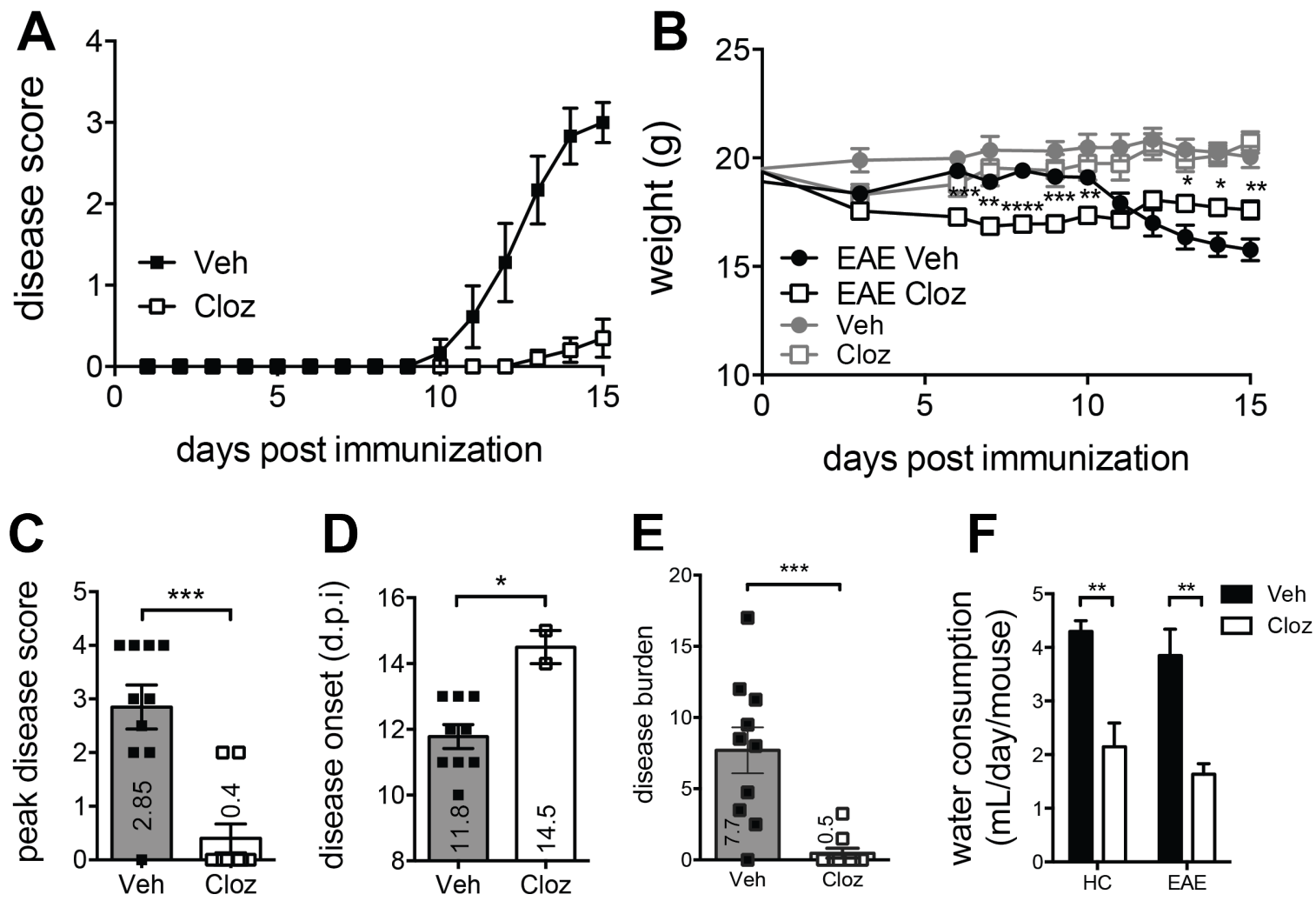

Figure 3.1 Clozapine treatment attenuates EAE disease course - day 15

A-F C57BL/6J mice begun treatment with clozapine (60 mg/kg/day) in their drinking water one day before EAE induction. Mice were scored (A) and weighed (B) daily for disease (0; normal - 5; moribund). Peak disease score of mice $(\mathbf{C})$. Disease onset of mice that succumbed to EAE up to 20 d.p.i (D) and overall disease burden assessed by area under the curve analysis (E). Daily water consumption averaged from three independent EAE experiments. (F). Shown are means and SEM of individual mice from two experiments combined ( $\mathrm{n}=10$ mice in each group; A-E). Statistical analyses performed by two-way ANOVA and Sidak's multiple comparison test for $\mathbf{B}$ and $\mathbf{F}$ comparing EAE Veh vs EAE Cloz. Mann-Whitney test for C-E. * p $<$ $0.05, * * \mathrm{p}<0.01, * * * \mathrm{p}<0.001, * * * * \mathrm{p}<0.0001$ 
Table 1. EAE disease parameters during clozapine treatment 15 d.p.i

\begin{tabular}{|c|c|c|c|c|c|}
\hline & Incidence & $\begin{array}{c}\text { Weight change \% } \\
\text { (day 15) }\end{array}$ & $\begin{array}{c}\text { Peak disease } \\
\text { score }\end{array}$ & Disease onset & Disease burden \\
\hline Vehicle & $90 \%$ & $\begin{array}{c}-17.30 \%+/-2.5 \% \\
(n=10)\end{array}$ & $\begin{array}{l}3.167+/-0.29 \\
(n=10)\end{array}$ & $\begin{array}{c}11.78+/-0.36 \\
(n=9)\end{array}$ & $\begin{array}{c}7.7+/-1.613 \\
(n=10)\end{array}$ \\
\hline Clozapine & $20 \%$ & $\begin{array}{c}-11.59 \%+/-2.79 \% \\
(n=10)\end{array}$ & $\begin{array}{c}0.4 * * *+/-0.27 \\
(n=10)\end{array}$ & $\begin{array}{c}14.5^{*}+/-0.5 \\
(n=2)\end{array}$ & $\begin{array}{c}3.25^{*}+/-0.3425 \\
(n=10)\end{array}$ \\
\hline
\end{tabular}

When mice are left to progress to the chronic phase of disease at 20 d.p.i, clozapine treated mice maintain a similar attenuated disease course (Figure 3.2A) and weight change (Figure 3.2B) as experiments previously completed at 15 d.p.i. However, at the later stages of disease, clozapine treated mice reach a higher mean peak disease score (Figure 3.2C) as more clozapine treated mice succumb to clinical disease, with a mean disease onset of 16.1 d.p.i vs 11.1 for vehicle treated mice (Figure 3.2D). Overall reduction in disease burden was maintained (Figure 3.2E) and altogether indicates that clozapine effectively delays the onset of disease and reduces the severity of EAE equating to an overall reduced disease burden. 

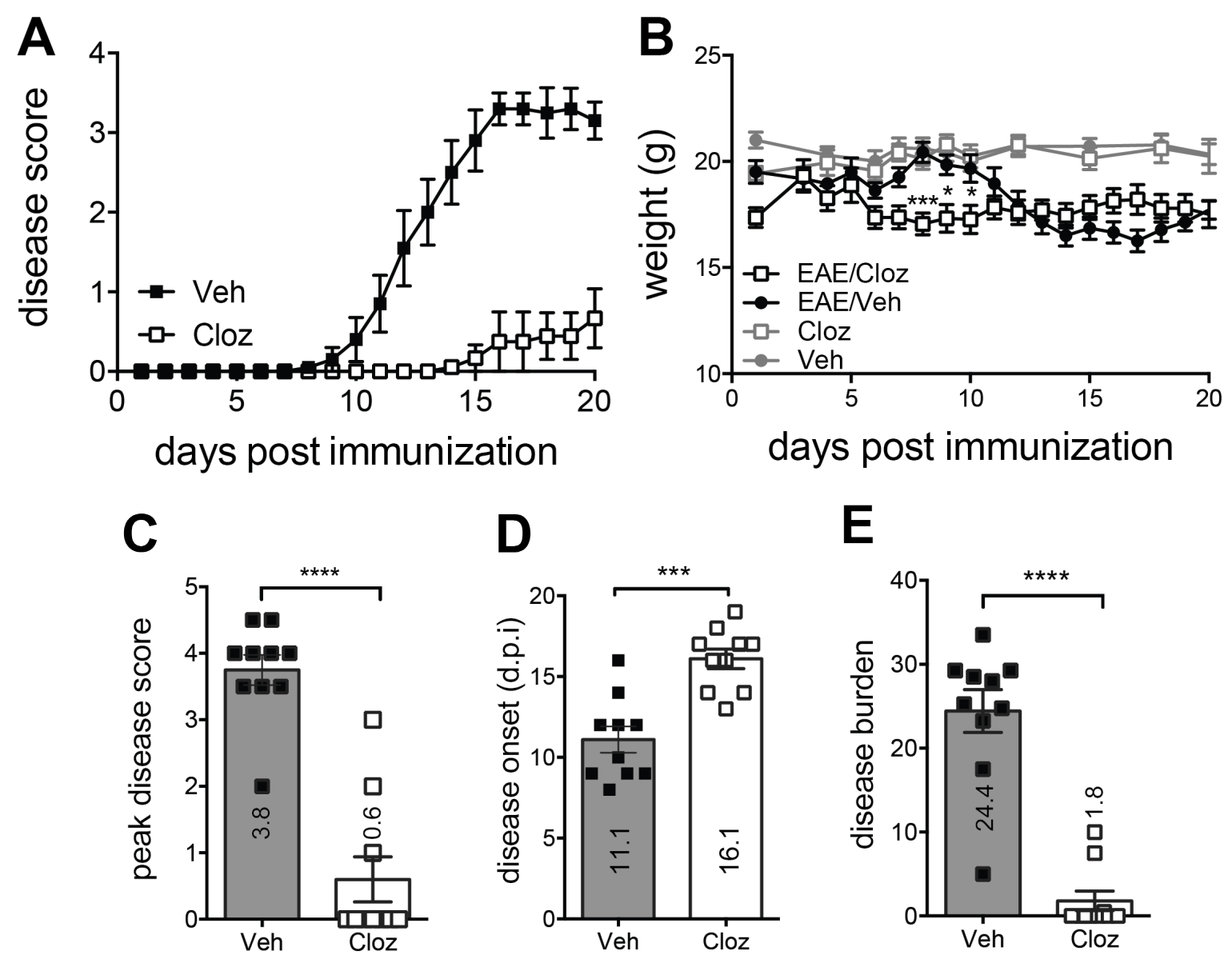

Figure 3.2 Clozapine attenuates EAE disease course - day 20

\begin{abstract}
A-E C57BL/6J mice begun treatment with clozapine $(60 \mathrm{mg} / \mathrm{kg} /$ day $)$ in their drinking water one day before EAE induction. Mice were scored (A) and weighed (B) daily for disease (0; normal - 5; moribund). Peak disease score of mice $(\mathbf{C})$. Disease onset of mice that succumbed to EAE up to 20 d.p.i (D) and overall disease burden assessed by area under the curve analysis $(\mathbf{E})$. Shown are means and SEM of individual mice from two experiments combined ( $\mathrm{n}=10$ mice in each group). Statistical analyses performed by Student's t-test $* * * \mathrm{p}<$ 0.001 and $* * * * \mathrm{p}<0.0001$
\end{abstract}

To investigate whether protection from disease by clozapine treatment is associated with altered peripheral immune cell subsets, spleens were analysed for changes in the lymphocyte and myeloid cell compartments by flow cytometry. During the induction phase of disease (5 d.p.i), neither EAE induction or clozapine treatment had a significant effect on the total number of cells in the spleen (Figure 3.3A). At this time, EAE induction caused a significant reduction in CD4 T cells in vehicle treated mice and to a lesser extent, clozapine treated mice (Figure 3.3B). Similar to the CD4 T cells, CD8 T cells were significantly reduced with EAE induction in vehicle treated mice and to a lesser extent in clozapine treated mice (Figure 3.3C). In contrast to CD4 T cells and CD8 T cells, CD1 $1 \mathrm{~b}^{+} \mathrm{SSC}^{\text {hi }}$ myeloid cells expand significantly with EAE induction with no difference between vehicle and clozapine treated mice (Figure 3.3D). 
During the effector phase of disease, neither EAE induction or clozapine treatment affected the total number of cells in the spleen (Figure 3.3E). Similar to the induction phase, CD4 and CD8 $\mathrm{T}$ cells are significantly lower in mice induced for EAE, however, the difference in CD4 T cells and CD8 T cells between vehicle and clozapine treated EAE mice is no longer detectable (Figure 3.3F and G). $\mathrm{CD}_{11} \mathrm{~b}^{+} \mathrm{SSC}^{\text {hi }}$ myeloid cells remain significantly higher in mice induced for EAE during the effector phase of EAE but no effect is observed with clozapine treatment (Figure 3.3H). These results are consistent with our recent work (L. Green et al., 2017) and indicates that although clozapine is highly effective at reducing EAE disease, little to no difference is observed on splenic immune cell subsets. 

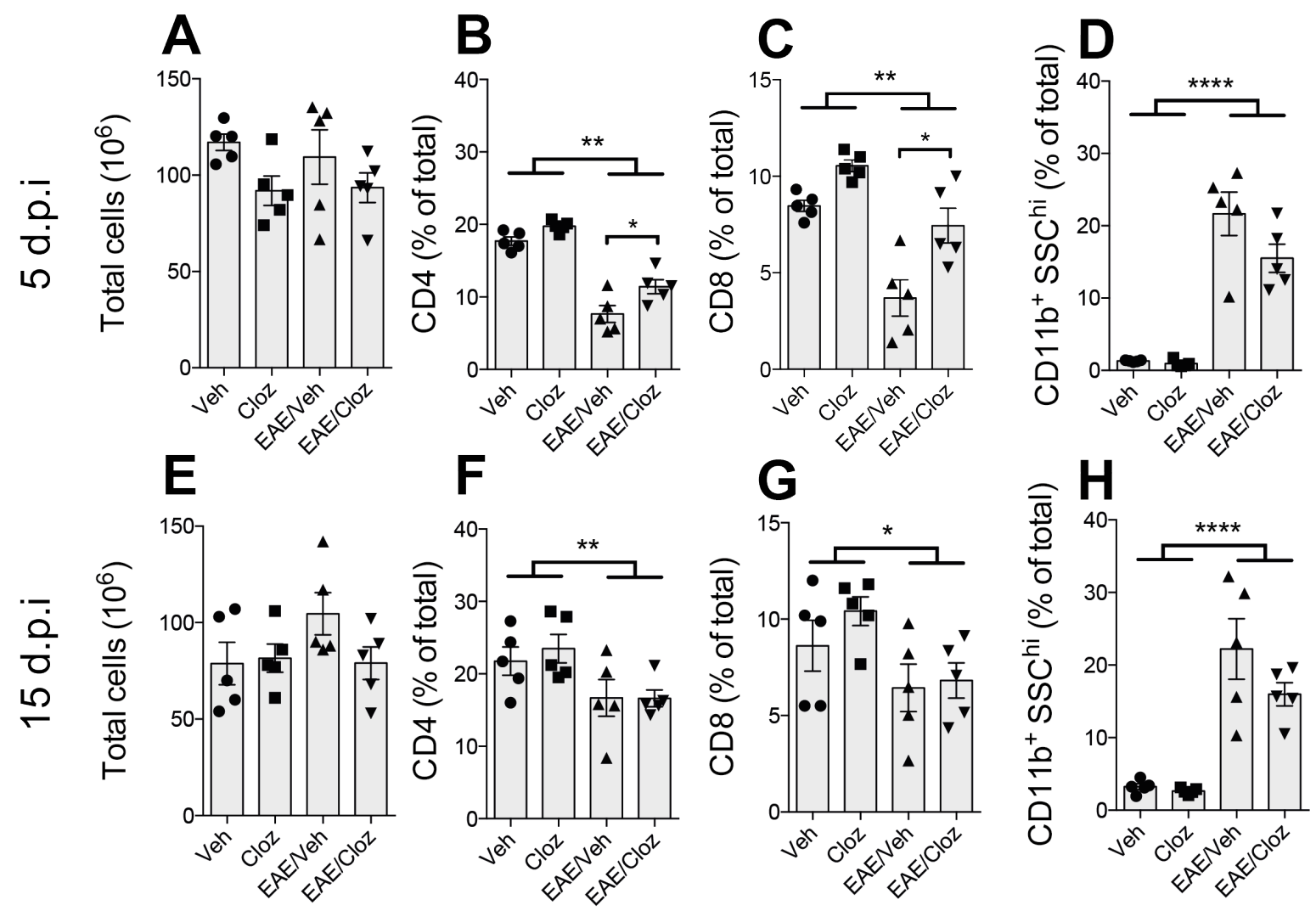

\section{Figure 3.3 Effects of clozapine on splenic immune cells}

EAE was induced in C57BL6/J mice treated with vehicle or clozapine (60 mg/kg/day). Spleens were isolated during the induction phase (5 d.p.i; A-D) or the effector phase (15 d.p.i; E-F) and analysed for immune cells by flow cytometry. A Total spleen cells 5 d.p.i determined by cell counting using a haemocytometer. B CD4 $\mathrm{T}$ cells as percentage of total splenocytes 5 d.p.i. C CD8 T cells as percentage of total splenocytes at 5 d.p.i. D $\mathrm{CD}_{11 \mathrm{~b}^{+}} \mathrm{SSC}^{\mathrm{hi}}$ myeloid cells as percentage of total splenocytes at 5 d.p.i. E Total spleen cells 15 d.p.i determined by cell counting using a haemocytometer. F CD4 T cells as percentage of total splenocytes 15 d.p.i.

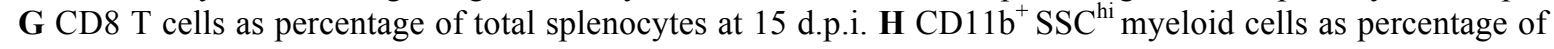
total splenocytes at 15 d.p.i. Shown are means and SEM of individual mice from one experiment at 5 d.p.i and another at 15 d.p.i ( $\mathrm{n}=5$ mice per group). Statistical analyses by two-way ANOVA and Sidak's multiple comparison test. $* \mathrm{p}<0.05, * * \mathrm{p}<0.01, * * * \mathrm{p}<0.001$ and $* * * * \mathrm{p}<0.0001$. 


\subsubsection{Clozapine administered after peak disease is effective at reducing disease in EAE}

While clozapine treatment started one day before the induction of EAE is effective at delaying and reducing disease, it was important to investigate how clozapine affects the recovery from EAE by starting treatment at the start of the chronic phase of EAE (20 d.p.i) (Figure 3.4A). Using this delayed, therapeutic treatment regimen, disease burden of vehicle and clozapine treated mice were measured overall from 0-40 d.p.i, before initiation of treatment 0-20 d.p.i and after from 20 d.p.i to 40 d.p.i. Although overall disease burden appears lower, it was not statistically significant $(\mathrm{p}=0.1772$; Figure 3.4B). Before treatment commenced, both experimental groups experienced a similar EAE disease course $(p=0.9670$; Figure 3.4A). When clozapine treatment commenced at 20 d.p.i, clozapine treatment appeared to improve slightly from before treatment $(p=0.1101)$ although this is not statistically significant. This experiment was performed as part of a larger study that was designed to have statistical power to detect this smaller improvement and when combined, these differences become statistically significant (see Appendix I) indicating that clozapine is effective at reducing disease during EAE even when given therapeutically (L. K. Green et al., 2017). 

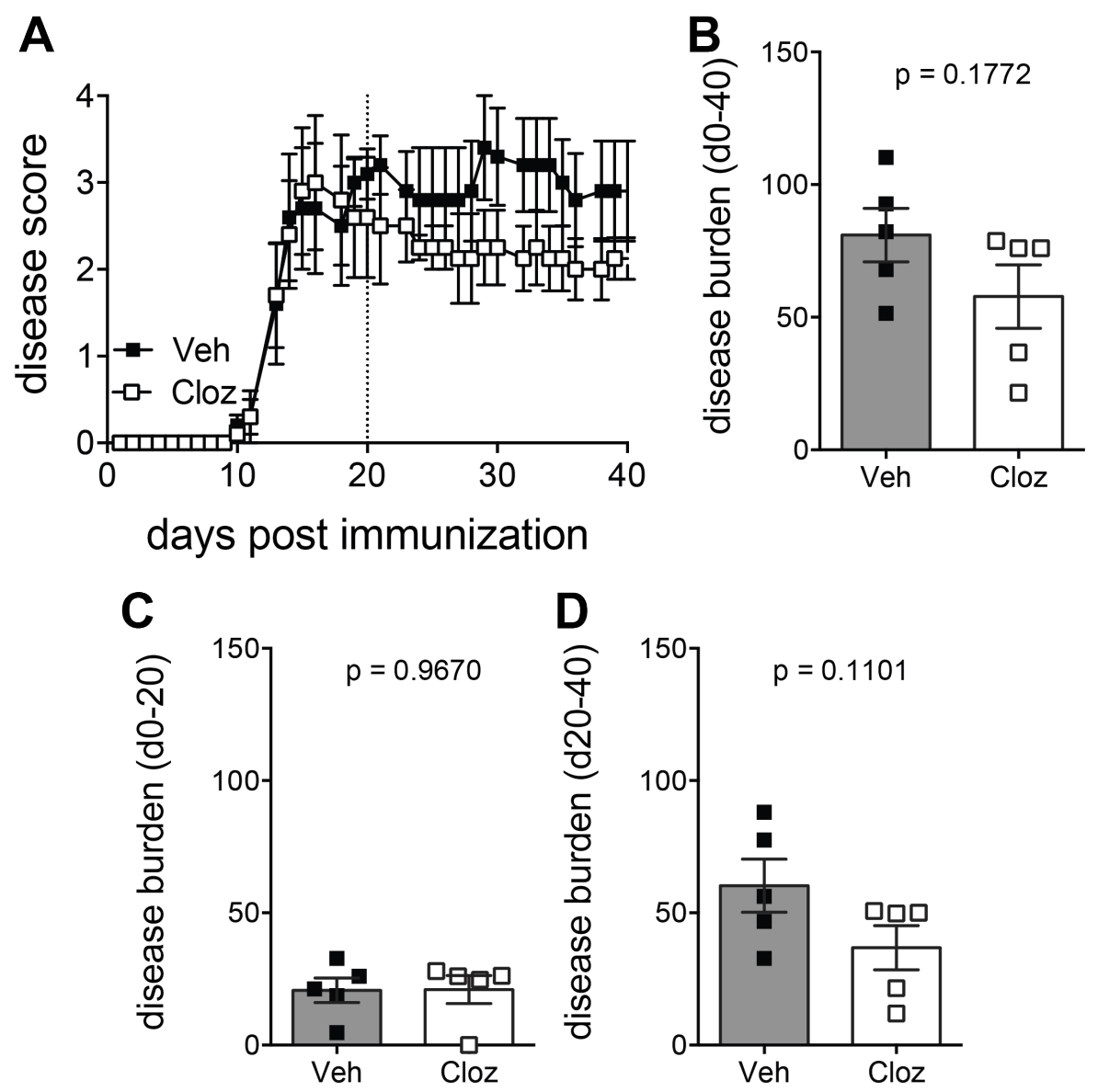

\section{Figure 3.4 Clozapine treatment after the induction phase of EAE is not effective at reducing disease}

A-D C57BL/6J mice were immunized to induce EAE and scored daily for disease (A) (0; normal - 5; moribund). Treatment with clozapine was started 20 d.p.i as indicated by dotted line in A. Overall disease burden analysed by area under the curve analysis between 0 d.p.i and 40 d.p.i (B). Disease burden of mice assessed by area under the curve between 0 d.p.i and 20 d.p.i. (C). Disease burden of mice assessed by area under the curve between 20 d.p.i and 40 d.p.i (D). Shown are means and SEM of individual mice from one experiment representative of three. Statistical analyses was performed by Student's t-test. 


\subsection{Discussion}

The aim of the experiments in this chapter was to validate and optimize previous findings from our laboratory showing that clozapine is effective at reducing disease severity during EAE when given during the induction phase of disease or after disease onset. We have previously shown that at a dose of $60 \mathrm{mg} / \mathrm{kg} /$ day, clozapine is highly effective at reducing EAE disease when administered by dissolving compounds in drinking water to achieve prolonged drug exposure (D. O'Sullivan et al., 2014). In these studies, administration of clozapine via drinking water was chosen as the more suitable route for investigating the effects of clozapine as it is highly effective at reducing EAE disease. Although clozapine treated mice lose weight after starting the treatment regimen, they maintain their weight throughout the experiment even when vehicle treated mice begin to lose more weight as they succumb to disease. In addition, clozapine treated mice do not visually appear distressed under clozapine treatment and is an acceptable compromise for an effective therapeutic. Future studies could incorporate a treatment regimen that uses oral gavage to administer a precise amount of clozapine each day in a similar manner to a human taking a tablet in a clinical setting.

Atypical antipsychotic use is heavily associated with weight gain in humans and obesity is a significant health-risk for cardiovascular disease, some cancers, diabetes and metabolic syndromes (Seidell, 2010). Evidence suggests that people taking antipsychotic agents long term have a higher mortality rate than those that do not (Weinmann, Read, \& Aderhold, 2009); and certainly, people suffering from schizophrenia have a higher mortality rate than the general population (Casey et al., 2004). The increased mortality rate is thought to be related to weight gain, although there is currently no conclusive evidence for this. Nevertheless, it is an important side effect of antipsychotic agents to consider when treating patients. We did not measure a significant increase in weight gain when treating mice with clozapine during these experiments and may suggest that at doses effective at reducing EAE weight gain is not a significant factor. In saying that, it cannot be excluded that the weight gain may have been offset by the initial fall in weight after adding clozapine to their drinking water.

Multiple sclerosis is usually identified after the onset of certain symptoms followed by a treatment regimen (Tsang \& Macdonell, 2011). Therefore, a therapeutic model of disease must be demonstrated to assess the efficacy of clozapine after the onset of disease. In this model, mice are treated during the chronic phase of disease after the initial peak in disease severity. We show that clozapine is indeed effective at reducing EAE disease when used therapeutically, 
indicating that clozapine has potential to treat MS therapeutically. This effect may appear modest, however, the EAE induction protocol induces massive infiltration of the CNS and rapid demyelination that reaches peak clinical score at 15 days post immunization causing neurological damage that is permanent (Papadopoulos, Pham-Dinh, \& Reynolds, 2006; Wujek et al., 2002). The fact that clozapine is able to significantly reduce disease even when given 20 d.p.i is remarkable and may therefore be a promising therapeutic for MS.

Analysis of peripheral immune cell subsets in the spleen of mice indicates that clozapine may alter the $\mathrm{T}$ cell compartment peripherally during the induction phase of disease. Although no significant changes between vehicle and clozapine treated mice were measured during the effector phase of disease, it is still possible that the differentiation state of $\mathrm{T}$ cells is affected in a way that could reduce EAE disease and this possibility is investigated in the following chapter.

\subsection{Conclusions}

The experiments in this chapter show that clozapine is effective at reducing overall disease severity during EAE when given before EAE induction or after peak disease and indicates that clozapine may be effective at treating MS and warrants clinical investigation for efficacy. In addition, an orally administered dose of $60 \mathrm{mg} / \mathrm{kg} / \mathrm{day}$ is highly effective at reducing EAE disease and will be used in the following chapters to investigate the immunological mechanisms involved in disease protection. Finally, these results show that clozapine has potential to alter peripheral immune cell subsets and is investigated in more detail as a potential mechanism by which it may reduce EAE disease in the following chapters. 
Chapter 4: The effects of clozapine on CD4 $T$ cell function 


\subsection{Introduction}

Atypical antipsychotic agents have recently been recognized for their immune modulating properties. It was shown in Chapter Three that clozapine modifies the disease course of experimental autoimmune encephalomyelitis (EAE), an immune-mediated demyelinating disease of the central nervous system (CNS). A number of published studies have already shown that atypical antipsychotic agents like clozapine can suppress the activation of microglia and reduce inflammatory serum cytokines; however, these studies alone do not sufficiently explain how clozapine affects inflammation in the brain during psychiatric disease like schizophrenia, nor do they explain how clozapine is able to modify the complex disease course of EAE. Given that clozapine is effective at reducing disease and delaying the onset of EAE, this chapter explores the effect of clozapine on the induction of the immune response with a focus on the development of antigen-specific CD4 T cell responses in the spleen to represent immune responses in the periphery as EAE is $\mathrm{T}$ cells are activated and differentiate in peripheral lymph nodes after subcutaneous immunisation (Fletcher et al., 2010). Splenocyte re-stimulation assays are commonly used as a measure of $\mathrm{T}$ cell priming efficacy and magnitude of response (Ciabattini, Pettini, \& Medaglini, 2013).

Although inflammatory damage in the CNS during multiple sclerosis (MS) and EAE is regulated by many cell types including resident microglia and infiltrating monocytes; one of the most crucial initiators of disease are myelin-reactive T cells. In EAE, an adaptive immune response to the self-antigen, myelin oligodendrocyte glycoprotein (MOG), is induced and this response is essential for disease manifestation. In healthy mice, only a few T cells express a given TCR that recognizes a specific antigen but upon activation, this small population rapidly expands and differentiates such that it dominates the immune repertoire. Activated $\mathrm{T}$ cells will differentiate into distinct effector lineages, and in the case of MOG-reactive $T$ cells, these activated $\mathrm{T}$ cells traffic across the $\mathrm{BBB}$ to initiate and regulate inflammation in their own distinctive fashion. It is well understood that these antigen-specific T cells are crucial for EAE and that both IFN- $\gamma$-producing Th1 and IL-17-producing Th17 subsets are crucial for disease induction and progression (Fletcher et al., 2010). This requirement was previously shown in IL-12p40 deficient mice, which fail to develop Th1 and Th17 responses and are consequently resistant to EAE (Gran et al., 2002). In contrast to pathogenic Th1 and Th17 subsets, regulatory $\mathrm{T}$ cells (Treg) maintain tolerance and act to limit effector $\mathrm{T}$ cell activation and proliferation, preventing excessive and inappropriate inflammatory damage. As expected, suppressing or 
deleting Treg function results in multi-organ autoimmunity in mice and humans and exacerbates EAE. In contrast, increasing Treg function by adoptive transfer of myelin-specific Treg can effectively reduce EAE disease (Kohm, Carpentier, Anger, \& Miller, 2002; L. A. Stephens, Malpass, \& Anderton, 2009; Xingmin Zhang et al., 2004).

Many of the available approved treatments for MS are immune modifying agents that alter various aspects of this complex disease. For example, the anti $\alpha 4 \beta 1$-integrin monoclonal antibody (Natalizumab) first developed using the EAE model blocks the migration of lymphocytes into the central nervous system, leading to a reduction in clinical disease (Ted A. Yednock et al., 1992). In humans, Natalizumab effectively reduces relapses in MS patients up to $68 \%$ (Polman et al., 2006). Other treatments such as glatiramer acetate target lymphocytes in a different manner and is thought to compete with myelin antigens on APC that promote Th2 (Duda, Schmied, Cook, Krieger, \& Hafler, 2000; H. J. Kim et al., 2004; Neuhaus et al., 2000; Vieira, Heystek, Wormmeester, Wierenga, \& Kapsenberg, 2003) and Treg (Hong, Li, Zhang, Zheng, \& Zhang, 2005), leading to suppression of harmful Th1 responses. The approach of altering $\mathrm{T}$ cell differentiation to treat autoimmune and inflammatory disease have been investigated using compounds such as rapamycin to inhibit key signalling enzymes that are differentially required for Th cell differentiation. Rapamycin inhibits the mammalian target of rapamycin (mTOR) pathway and this inhibits the differentiation of Th1 and Th17 cells while promoting differentiation to Treg $(\mathrm{Chi}, 2012)$. This approach has proven effective for reducing disease in EAE (Esposito et al., 2010). In addition, GSK-3 $\beta$ signalling downstream of Akt has also been shown to be a key regulator in the differentiation of Th1 and Th17 cells (Beurel et al., 2013; E. Beurel, W. I. Yeh, S. M. Michalek, L. E. Harrington, \& R. S. Jope, 2011). GSK$3 \beta$ is of particular interest to this study for it has been proposed as the therapeutic target of atypical antipsychotics such as risperidone and clozapine in psychiatric illnesses as typical antipsychotics do not share these properties (Li, Rosborough, Friedman, Zhu, \& Roth, 2007; Roh et al., 2007). GSK-3 $\beta$ is constitutively active and phosphorylates $\beta$-catenin, tagging it for ubiquitination and degradation (Figure 4.1A). Therefore, $\beta$-catenin expression can be used as a measure of GSK-3 $\beta$ activity. Therefore, if clozapine inhibits GSK-3 $\beta$ in our model systems, we would expect $\beta$-catenin to accumulate (Figure 4.1B). 
A

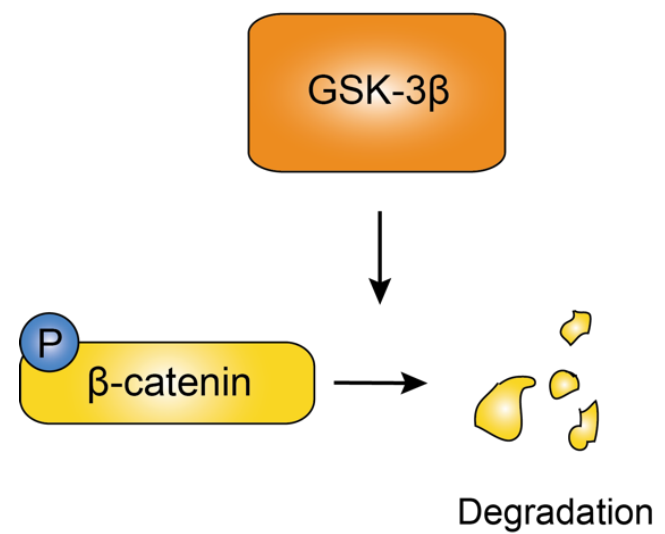

B

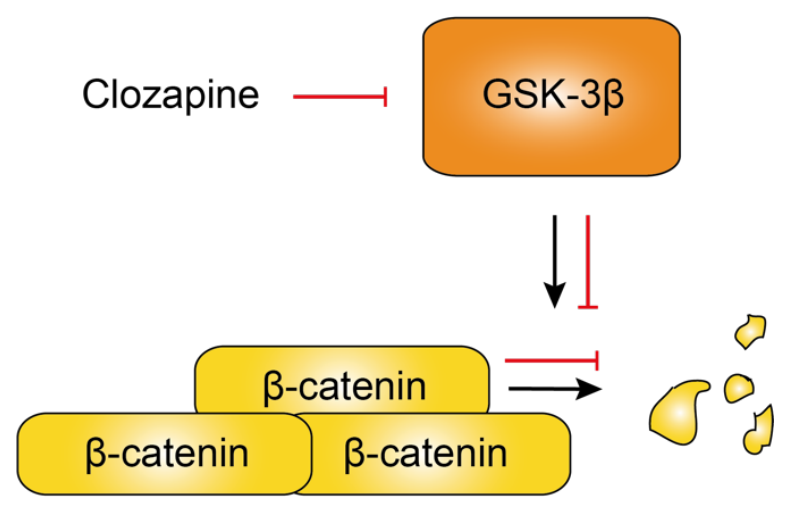

Figure 4.1 GSK-3 $\beta$ regulates $\beta$-catenin expression

A The constitutively active enzyme GSK-3 $\beta$ regulates $\beta$-catenin expression by continually phosphorylating $\beta$ catenin to tag it for degradation in the proteasome. B It is proposed that if clozapine inhibits GSK-3 $\beta$ in our model systems, $\beta$-catenin would accumulate within cells.

Another effective method of modifying EAE disease is by suppressing the proliferation of selfreactive lymphocytes. Mitoxantrone achieves this by preventing mitosis through disrupting DNA synthesis (Kapuscinski \& Darzynkiewicz, 1986) and this effectively reduces disease in both EAE (Ridge et al., 1985) and MS (Jeffery \& Herndon, 2004). These studies show that EAE disease can be attenuated by targeting $\mathrm{T}$ cell proliferation and altering the $\mathrm{T}$ helper cell differentiation.

Since the discovery that clozapine and other atypical antipsychotic agents are immunomodulatory was fairly recent, limited information is available regarding the effects of clozapine on the induction of a complex immune response involving both the innate and adaptive immune systems. Currently, the idea that clozapine inhibits the activation of microglia does not definitively explain its mechanism of action in suppressing CNS inflammation, specifically during a $\mathrm{T}$ cell-mediated disease like EAE.

This chapter aims to elucidate the effects of clozapine treatment on the proliferation of myelinreactive $\mathrm{T}$ cells, their differentiation into distinct $\mathrm{T}$ helper cell lineages and effector capacity to induce EAE disease, as a potential mechanism by which it may reduce disease during EAE. 


\subsection{Aim}

The experiments performed in this chapter aim to understand the effect of atypical antipsychotic agents on the activation, differentiation and expansion of $\mathrm{CD}^{+} \mathrm{T}$ cells using in vitro and in vivo techniques to understand the mechanism by which these agents may be working to delay the onset of disease and reduce severity during EAE.

\subsubsection{Specific Aims}

1. To determine whether clozapine treatment has any effect on the differentiation and expansion of $\mathrm{CD} 4^{+} \mathrm{T}$ cells

2. To determine whether changes in the in vitro model translate to in vivo changes during EAE.

3. To study the effects of clozapine on antigen-specific T cell proliferation in vivo after immunization with $\mathrm{MOG}_{35-55}$-peptide to induce $\mathrm{EAE}$ 


\subsection{Results}

\subsubsection{Clozapine reduces the viability of $\mathrm{CD}^{+} \mathrm{T}$ cells in a concentration dependent manner}

In order to appropriately assess the effect of clozapine on CD4 T cell responses, a viability assay was conducted to define an appropriate concentration of clozapine that was not cytotoxic

to cells cultured and stimulated in vitro. Briefly, splenocytes from $2 \mathrm{D} 2^{\mathrm{MOG} 35-55} \mathrm{TCR}$ mice were stimulated with $\mathrm{MOG}_{35-55}$ peptide in increasing concentrations of clozapine for 72 hours. Under these conditions, $\mathrm{CD}^{+} \mathrm{T}$ cells from unstimulated wells did not survive regardless of the presence of clozapine in the culture medium indicating that $\mathrm{CD} 4^{+} \mathrm{T}$ cells did not remain viable in cell culture without stimulation (Figure 4.2A-B). $\mathrm{MOG}_{35-55}$-peptide-stimulated splenocytes had expanded $\mathrm{CD}^{+} \mathrm{T}$ cell proportions as we would expect, and when clozapine was added to the culture medium, there was a significant reduction in viable CD4 T cells above $40 \mu \mathrm{M}$ (Figure 4.2B) but remained viable at $20 \mu \mathrm{M}$ or less for up to five days of stimulation (Figure 4.2C). Therefore, a clozapine concentration of $20 \mu \mathrm{M}$ was used for all subsequent in vitro experiments. 

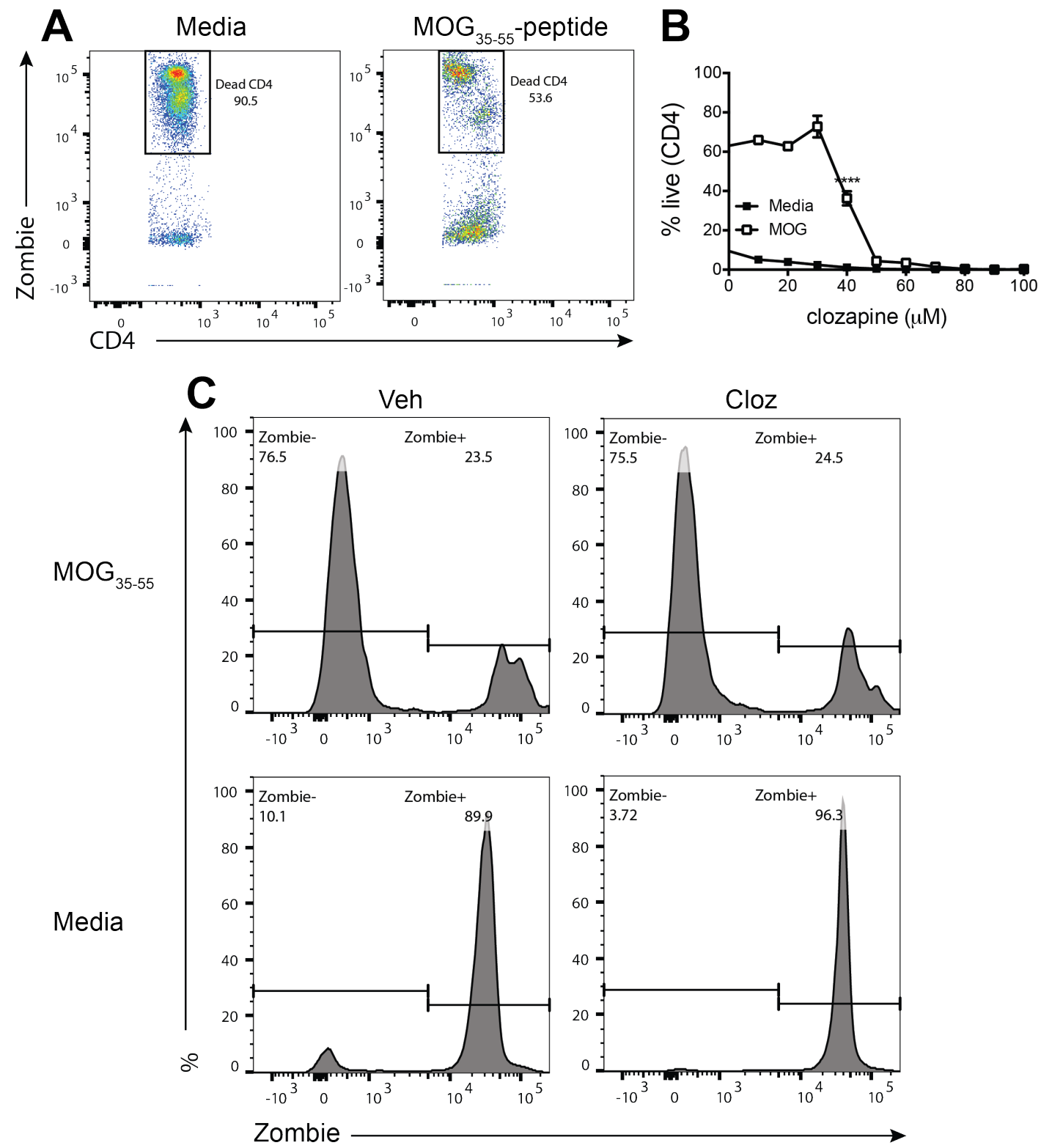

\section{Figure 4.2 Clozapine reduced viability of stimulated CD4 $\mathrm{T}$ cells at concentrations greater than $40 \mu \mathrm{M}$}

A-C 2D2 splenocytes were stimulated with $\mathrm{MOG}_{35-55}$-peptide for 72 hours (A and B) or 120 hours (C) and analysed for cell viability by flow cytometry using Zombie NIR viability dye. (A) Representative dot plots of Zombie NIR staining in CD4+ T cells (gated to exclude doublets) after 72 hours with $\mathrm{MOG}_{35-55}$-peptide. (B) Graph of CD4 T cell viability in increasing concentrations of clozapine. Shown are means and SEM of duplicate wells representing two experiments. $* * * * p<0.001$ by one-way ANOVA and Dunnett's multiple comparison test. (C) Zombie NIR histograms of CD4 T cells from pooled triplicate wells of 2D2 splenocytes stimulated with $\mathrm{MOG}_{35-55}$-peptide after 120 hours in clozapine $(20 \mu \mathrm{M})$ or vehicle from one experiment. 


\subsubsection{Clozapine did not alter Th1 and Th17 differentiation but promoted Treg expansion}

In light of the signalling alterations clozapine is reported to modify, it was hypothesized that clozapine could alter signalling pathways important for translating cytokine signals during CD4 $\mathrm{T}$ cell differentiation into effector Th1, Th17 or Treg. To generate activated antigenspecific $\mathrm{T}$ cells biased towards different subset lineages, 2D2 $2^{\mathrm{MOG} 35-55} \mathrm{TCR}$ splenocytes were stimulated with the $\mathrm{MOG}_{35-55}$ peptide in vitro. Firstly, to determine whether this model could effectively differentiate $\mathrm{MOG}_{35-55-s p e c i f i c} \mathrm{CD}^{+} \mathrm{T}$ cells into Th1, Th17 and Treg subsets, 2D2 ${ }^{\mathrm{MOG} 35-55}$ TCR splenocytes were stimulated with $\mathrm{MOG}_{35-55}$ peptide alone, and the expression of transcription factors T-bet, ROR $\gamma \mathrm{T}$ and Foxp3 were measured in T cells by flow cytometry (Figure 4.3A-C). This environment induced moderate T-bet expression (Figure 4.3D) but did not induce expression of $\operatorname{ROR} \gamma \mathrm{T}$ (Figure 4.3E) or Foxp3

Figure 4.3F). When stimulated in Th1 differentiating medium, T-bet expression (i.e. MFI) increased by $97 \%$ over $\mathrm{MOG}_{35-55}$-peptide alone (Figure 4.3D) indicating robust differentiation to Th1. Stimulating 2D2 $2^{\text {MOG35-55 }}$ TCR splenocytes in Th17 differentiating medium, increased ROR $\gamma$ T expression by 111\% (Figure 4.3E), whereas T-bet (Figure 4.3D) and Foxp3 (Figure 4.3F) were not induced indicating robust Th17 differentiation. Finally, when stimulated in Treg differentiating medium, Foxp3 expression increased by $160 \%$ over $\mathrm{MOG}_{35-55}$-peptide alone (Figure 4.3F) with no induction of T-bet (Figure 4.3D) or ROR $\gamma \mathrm{T}$ (Figure 4.3E) indicating robust differentiation to iTreg. 


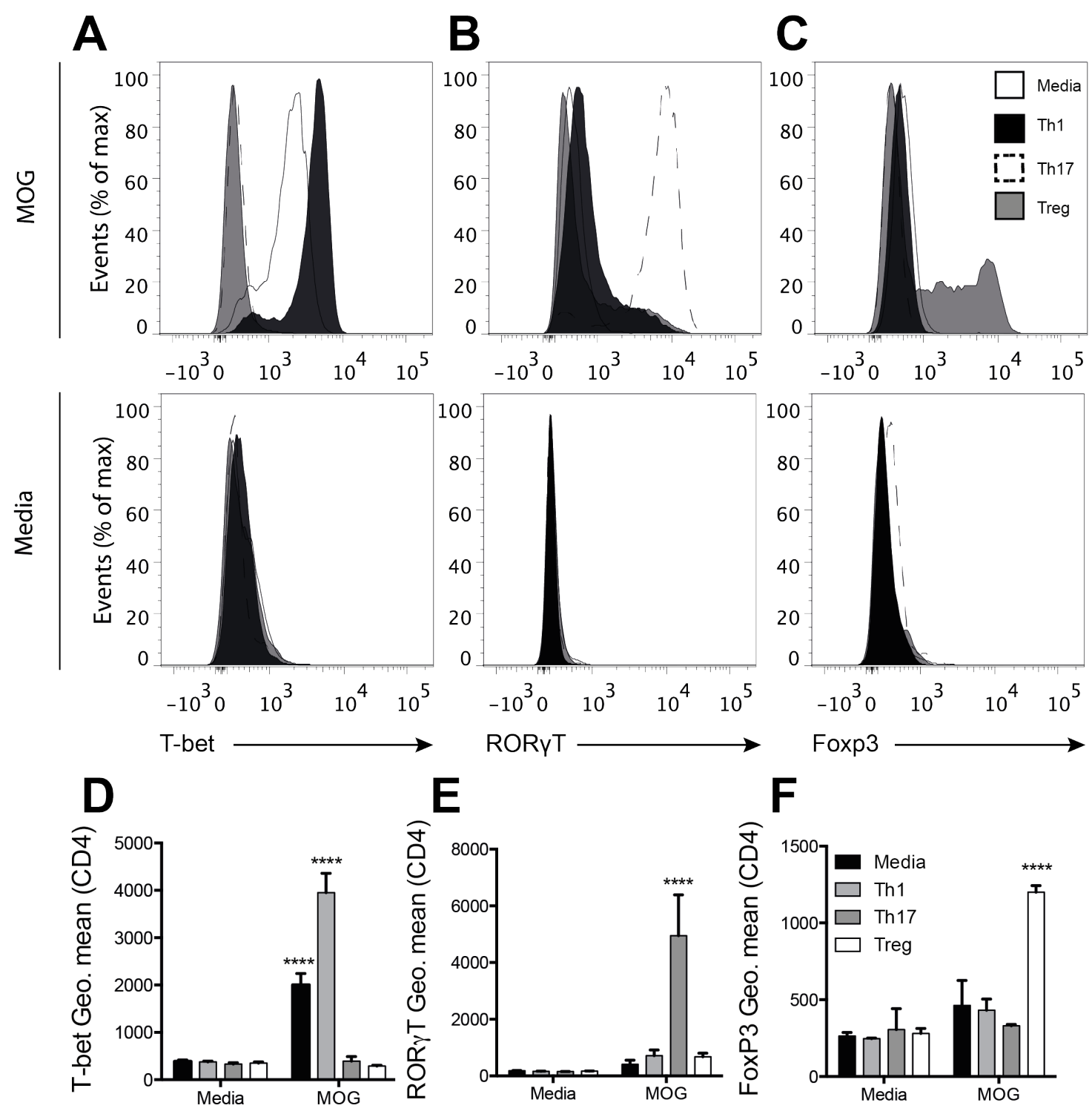

Figure 4.3 Induction of T-bet, ROR $\gamma \mathrm{T}$ and Foxp3 in CD4 T cells

A-F 2D2 splenocytes were plated at $1 \times 10^{6}$ cells $/ \mathrm{mL}$ and stimulated with $\mathrm{MOG}_{35-55}$-peptide $(50 \mu \mathrm{g} / \mathrm{mL})$ in $\mathrm{rIL}-$ 12 p70 $(20 \mathrm{ng} / \mathrm{mL})$ and anti-IL-4 (11B11; $10 \mu \mathrm{g} / \mathrm{mL})$ for Th1 differentiation. rTGF $\beta 1(5 \mathrm{ng} / \mathrm{mL})$, rIL-6 (20 $\mathrm{ng} / \mathrm{mL})$ and anti-IFN $\gamma(\mathrm{XMG1} .2 ; 10 \mu \mathrm{g} / \mathrm{mL})$ for Th17 differentiation. rTGF $\beta 1(5 \mathrm{ng} / \mathrm{mL})$, anti-IL-6 (MP520F3; $10 \mu \mathrm{g} / \mathrm{mL})$ and anti-IFN $\gamma(\mathrm{XMG} 1.2 ; 10 \mu \mathrm{g} / \mathrm{mL})$ for Treg differentiation. A-C Histogram of transcription factor expression in CD4 T cells when cultured in medium or Th1 (A), Th17 (B) and Treg (C) differentiating conditions. D-F Graphical presentation of the geometric mean fluorescence intensity of transcription factors in CD4 T cells after differentiating in medium or Th1(D), Th17 (E) and Treg (F) differentiating conditions. Shown are the means and SEM of triplicate wells from one experiment representing three independent experiments. $* * * * \mathrm{p}<0.001$ by two-way ANOVA and Sidak's multiple comparison test. Gating strategy to identify CD4 T cells are shown in Appendix II.

Clozapine was then added to the culture during differentiation to Th1 at $20 \mu \mathrm{M}$, which is the concentration previously determined not to cause cell death, and analysed for Th1 differentiation by expression of T-bet and production of IFN- $\gamma^{+}$(Figure 4.4A). With clozapine 
present, no difference in IFN $-\gamma^{+}$cells was observed (Figure 4.4B). Clozapine did, however, prompt a subtle but statistically significant decrease in the proportion of $\mathrm{T}^{-b e{ }^{+}}{ }^{+}$cells after differentiation (Figure 4.4C). The level of T-bet protein expression in CD4 T cells was not significantly altered by clozapine (Figure 4.4D) indicating that although there are less T-bet ${ }^{+}$ cells, T-bet expression within cells was unaffected. These results indicate that clozapine has a minor effect on the differentiation to Th1 cells, but whether this modest change results in a functional difference needs to be determined.
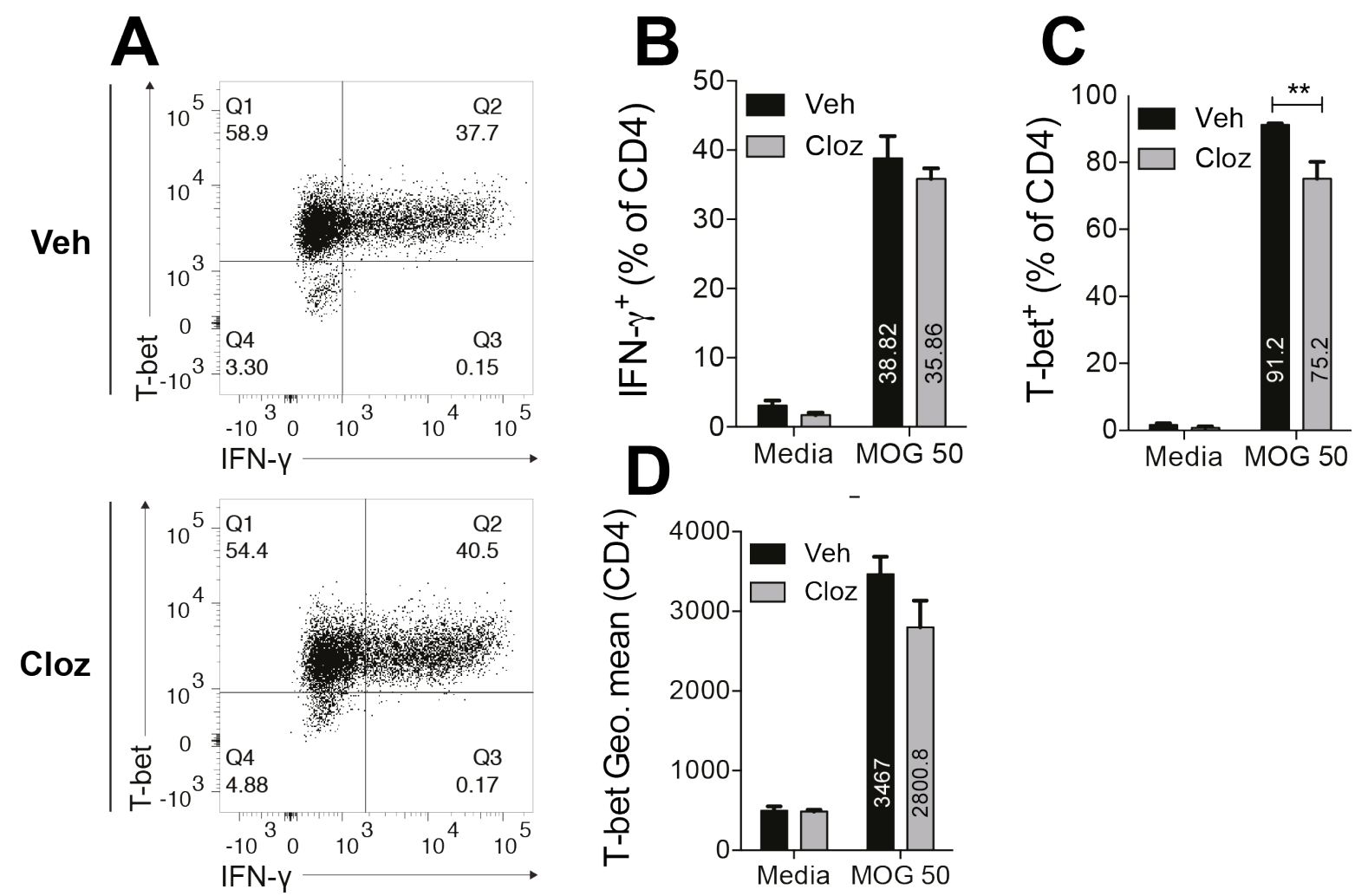

\section{Figure 4.4 Clozapine did not significantly alter differentiation to Th1}

A-D 2D2 $\mathrm{TCR}^{\mathrm{MOG} 35-55}$ splenocytes were cultured at $1 \times 10^{6}$ cells/well in Th1 differentiation medium $(20 \mathrm{ng} / \mathrm{mL}$ IL-12p70, $10 \mu \mathrm{g} / \mathrm{mL}$ anti-IL-4) and stimulated with $\mathrm{MOG}_{35-55}$ peptide for 72 hours. A Representative dot plots of T-bet and IFN- $\gamma$ expression in $\mathrm{CD}^{+} \mathrm{T}$ cells after differentiation with clozapine $(20 \mu \mathrm{M})$ or vehicle. Gating strategy to identify CD4 $\mathrm{T}$ cells is shown in Appendix II. B Proportion of IFN- $\gamma^{+}$CD4 $\mathrm{T}$ cells after differentiation. C Proportion of T-bet ${ }^{+}$CD4 cells after differentiation. D Geometric mean of T-bet in CD4 T cells. Shown are means and SEM of two independent experiments combined. Statistical analyses were performed by two-way ANOVA with Sidak's multiple comparison test. 
Differentiation of CD4 T cells towards Th17 requires TCR activation and the cytokine signals IL-6 and TGF- $\beta$. Clozapine was added to the culture medium during differentiation to Th17 cells and ROR $\gamma \mathrm{T}$ expression and IL-17A production were assessed (Figure 4.5A). Similar to

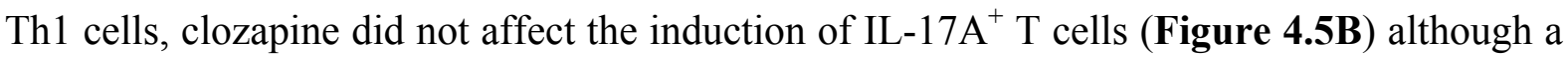
subtle but statistically significant reduction in the proportion of $\mathrm{ROR} \gamma \mathrm{T}^{+} \mathrm{CD} 4$ cells was observed (Figure 4.5C). Despite a slight reduction in $\mathrm{ROR} \gamma \mathrm{T}^{+} \mathrm{CD} 4$ cells, no reduction in the expression level of ROR $\gamma \mathrm{T}$ transcription factor was measured in CD4 cells (Figure 4.5D). These results indicate that clozapine does not prevent differentiation to Th17 cells.
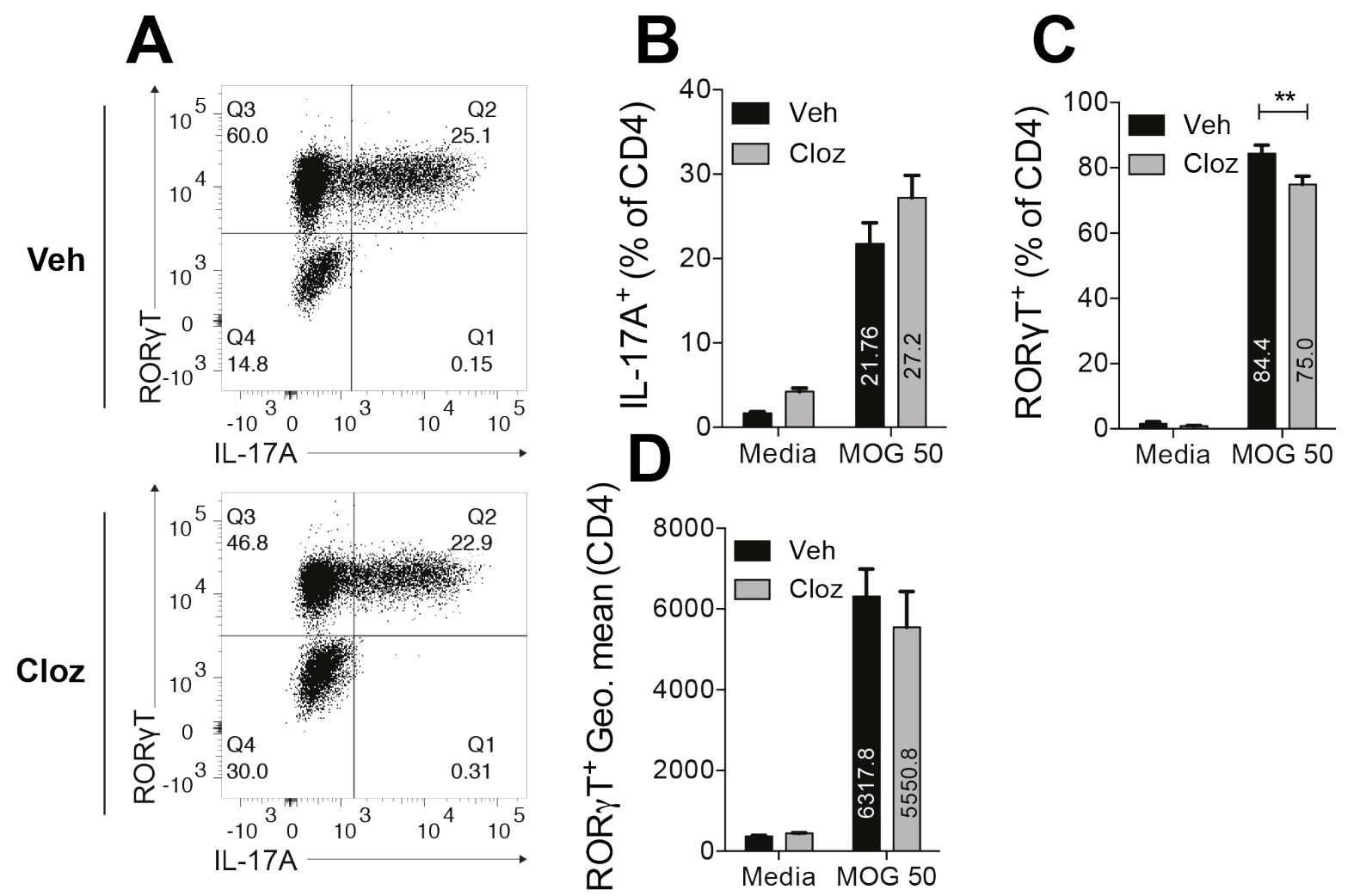

\section{Figure 4.5 Clozapine did not alter Th17 differentiation}

A-D 2D2 TCR ${ }^{\text {MOG35-55 }}$ splenocytes were cultured at $1 \times 10^{6}$ cells/well in Th17 differentiating medium $(5 \mathrm{ng} / \mathrm{mL}$ TGF- $\beta, 20 \mathrm{ng} / \mathrm{mL}$ IL- 6 and $10 \mu \mathrm{g} / \mathrm{mL}$ anti IFN- $\gamma$ ) and stimulated with $\mathrm{MOG}_{35-55}$ peptide for 72 hours. A ROR $\gamma \mathrm{T}$ and IL-17A expression in $\mathrm{CD}^{+} \mathrm{T}$ cells after differentiation with clozapine $(20 \mu \mathrm{M})$ or vehicle. Rationale for setting quadrant gates can be found in Appendix II. B Proportion of IFN- $\gamma^{+}$CD4 T cells after Th1 differentiation. C Proportion of ROR $\gamma \mathrm{T}^{+} \mathrm{CD} 4 \mathrm{~T}$ cells after Th17 differentiation. D Geometric mean of ROR $\gamma \mathrm{T}$ in CD4 T cells. Shown are means and SEM of two independent experiments combined. Statistical analyses were performed by two-way ANOVA and Sidak's multiple comparison test.

In order for iTreg to differentiate, TGF- $\beta$ must be present and IL- 6 absent during TCR stimulation. To investigate whether clozapine affected iTreg differentiation, clozapine was 
added to the culture medium during stimulation. Activated iTreg were identified as CD25 Foxp $3^{+}$by flow cytometry (Figure 4.6A). Interestingly, clozapine increased the frequency of Foxp $3^{+} \mathrm{CD} 25^{+} \mathrm{CD} 4 \mathrm{~T}$ cells significantly from $33 \%$ to $50 \%$ (Figure 4.6B). In addition, clozapine increased the expression of Foxp3 in iTreg (Figure 4.6C) indicating that clozapine promoted the expression of Foxp3 and the expansion of Foxp $3^{+}$iTreg. Altogether, these data show that while clozapine had minor effects on $\mathrm{MOG}_{35-55}$-specific differentiation to Th1 and Th17 cells, it effectively promoted iTreg differentiation.

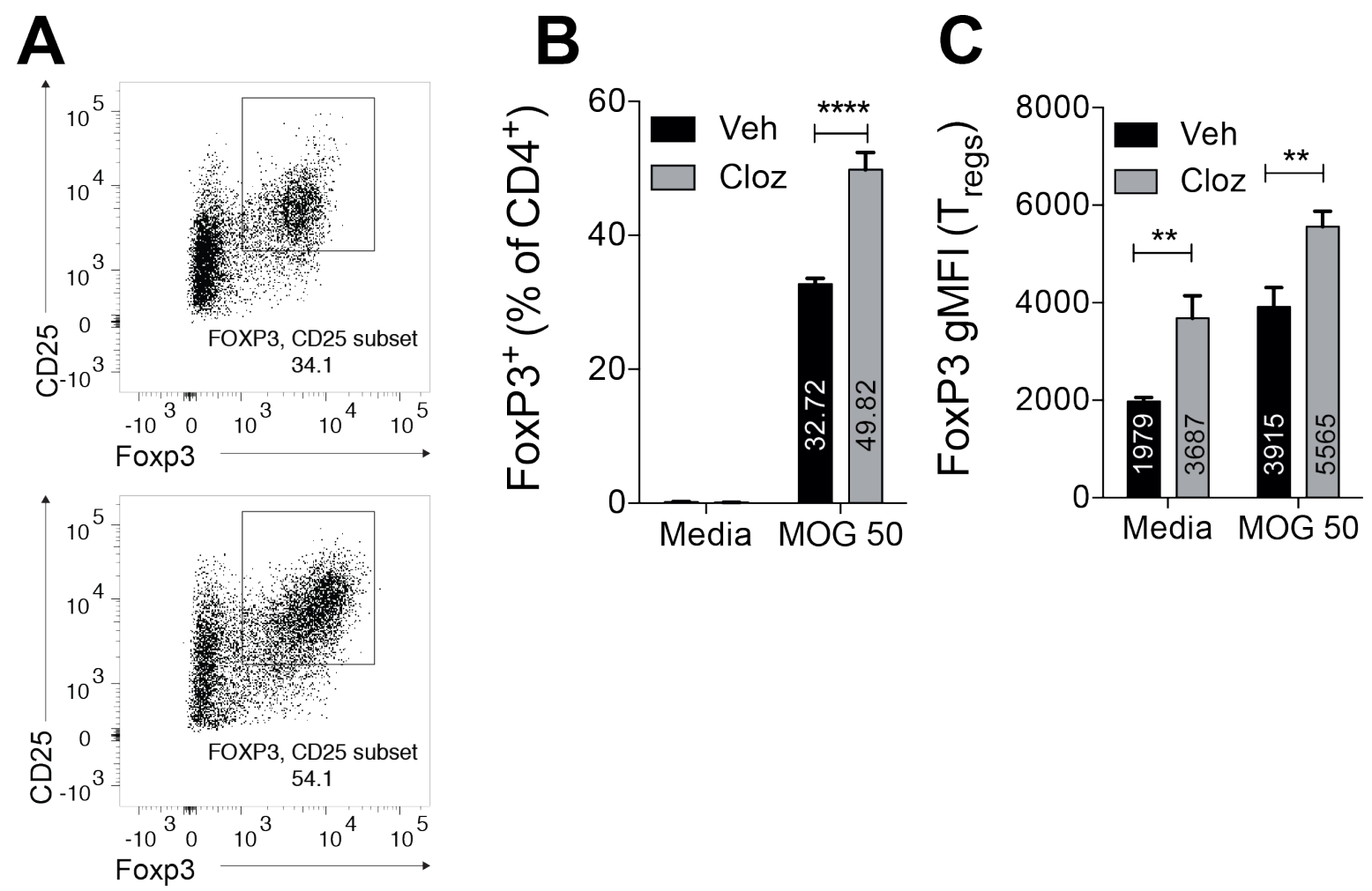

\section{Figure 4.6 Clozapine promoted Treg differentiation and Foxp3 expression}

A-C 2D2 splenocytes were plated at $1 \times 10^{6}$ cells $/ \mathrm{mL}$ and stimulated with $\mathrm{MOG}_{35-55}$-peptide $(50 \mu \mathrm{g} / \mathrm{mL})$ in Treg differentiating medium (rTGF $\beta 1(5 \mathrm{ng} / \mathrm{mL})$, anti-IL-6 (MP5-20F3; $10 \mu \mathrm{g} / \mathrm{mL})$ and anti-IFN- $\gamma(X M G 1.2 ; 10$ $\mu \mathrm{g} / \mathrm{mL})$ A Representative dot plots of CD25 and Foxp3 expression after differentiating in clozapine $(20 \mu \mathrm{M})$ or vehicle. Gating strategy to identify CD4 T cells are shown in Appendix II. B Proportion of Foxp3 ${ }^{+}$CD4 T cells after differentiation. C Geometric MFI of Foxp3 on Foxp $3^{+}$Treg after differentiation. Shown are means and SEM of two independent experiments combined. Statistical analyses were performed by two-way ANOVA with Sidak's multiple comparison test. 


\subsection{3 $\beta$-catenin expression was unaltered by clozapine in iTreg}

To investigate the potential signalling alterations that may have enhanced the differentiation of iTreg in the presence of clozapine, a pilot study was performed to investigate the involvement of GSK-3 $\beta$ by measuring $\beta$-catenin expression by flow cytometry in Treg and non-Treg as a measure of GSK-3 $\beta$. Firstly, the differentiation of iTreg was determined 24, 48 and 72 hours post stimulation. By 24 hours, iTreg were differentiated only in the presence of $\mathrm{MOG}_{35-55}$ peptide and Treg-inducing medium (Figure 4.7A). At this time point, clozapine did not affect the differentiation of iTreg as no difference was observed between the vehicle and clozapine conditions (Figure 4.7A). No iTreg differentiation was observed in the absence of $\mathrm{MOG}_{35-55^{-}}$ peptide even in Treg-inducing conditions from 24-72 hours (Figure 4.7A-C). By 48 hours, iTreg differentiation was increased further and clozapine induced a significant increase in the proportion of iTreg in the culture when compared to the vehicle control (Figure 4.7B). Similarly, after 72 hours, the proportion of iTreg continued to rise and the increase in iTreg in the clozapine condition was maintained (Figure 4.7C).

Next, $\beta$-catenin expression in iTreg (Foxp3+) and non-Treg (Foxp3-) CD4 T cells was compared (Figure 4.7D). $\mathrm{MOG}_{35-55}$-peptide stimulation induced an increase in $\beta$-catenin expression in cells stimulated in media or Treg-inducing conditions, and this was maintained from 24 to 72 hours (Figure 4.7E-F). Clozapine induced a modest but statistically significant increase in $\beta$-catenin expression in Foxp3- CD4 T cells after 48 hours (Figure 4.7E). No difference in $\beta$-catenin expression was detected in either iTreg or non-Treg cultured in the presence of vehicle or clozapine at 24 and 72 hours. Additionally, no difference in $\beta$-catenin expression was measured between iTreg and non-Treg irrespective of their culture conditions or time in culture (Figure 4.7E). These results indicate that iTreg do not have a higher expression of $\beta$-catenin than non-Treg and that increased iTreg differentiation by clozapine is not associated with an increase in $\beta$-catenin expression in CD4 T cells. 

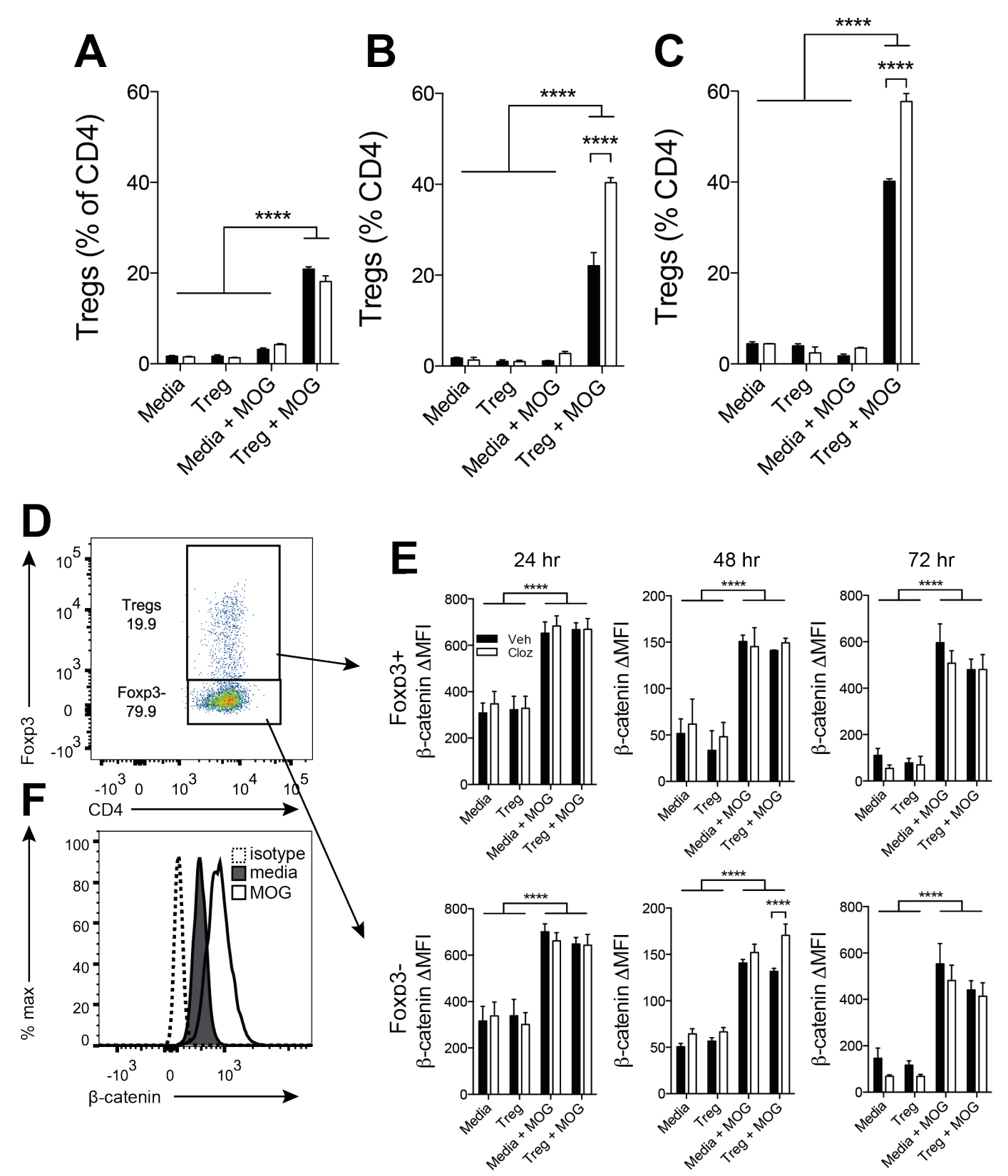

\section{Figure 4.7 $\beta$-catenin expression was unaltered by clozapine in iTreg}

A-E 2D2 splenocytes were plated at $1 \times 10^{6}$ cells $/ \mathrm{mL}$ and stimulated with $\mathrm{MOG}_{35-55}$-peptide $(50 \mu \mathrm{g} / \mathrm{mL})$ in Treg differentiating medium (rTGF $\beta 1(5 \mathrm{ng} / \mathrm{mL})$, anti-IL-6 (MP5-20F3; $10 \mu \mathrm{g} / \mathrm{mL})$ in the presence of clozapine (20 $\mu \mathrm{M})$ or vehicle. Media, media control; Treg, Treg inducing medium; $\mathrm{MOG}, \mathrm{MOG}_{35-55}$-peptide. A Foxp $3^{+}$Treg are presented as the percentage of CD4 after 24 hours (B), 48 hours, (C) or 72 hours of culture (D). Representative gating of strategy to identify Foxp3 ${ }^{+}$Treg and Foxp3 $3^{-} \mathrm{CD} 4 \mathrm{~T}$ cells to measure $B$-catenin expression. Gating strategy to identify CD4 T cells is shown in Appendix II. E $\beta$-catenin expression presented as $\triangle \mathrm{MFI}(\triangle \mathrm{MFI}=\mathrm{MFI}$ - isotype control). $\mathbf{F}$ Histogram of $\beta$-catenin expression of isotype control, unstimulated and $\mathrm{MOG}_{35-55}$-peptide stimulated CD4 T cells. Shown are means and SEM of triplicate wells from one experiment. $* * \mathrm{p}<0.01,{ }^{* * * *} \mathrm{p}<0.001$ by two-way ANOVA and Sidak's multiple comparison test. 


\subsubsection{Treatment with clozapine did not alter peripheral Th cell subsets during EAE}

Due to the finding that clozapine can alter Th1 and Th17 differentiation while promoting iTreg in an in vitro system, it was necessary to determine whether a similar effect on $\mathrm{T}$ cell differentiation could be observed in an in vivo model. To assess differentiation of $\mathrm{T}$ cell subsets in vivo, mice were immunized to induce $\mathrm{EAE}$ and $\mathrm{T}$ cell subset populations were measured during the induction (5 d.p.i) and the effector phases (15 d.p.i) of EAE. During the induction phase when all mice were sub-clinical (Figure 4.8A), Th17 differentiation was assessed by ROR $\gamma$ T expression in CD4 T cells. While immunization for EAE induced a significant increase in proportion of Th17 cells compared to unimmunized mice, no impairment in the induction of Th17 cells was detected during treatment with clozapine (Figure 4.8B). Treg were identified as $\mathrm{CD} 25^{+}$Foxp $^{+}$and at 5 d.p.i, the proportion of Treg were significantly lower in immunized mice compared to unimmunized controls. Clozapine treatment did not have an effect on the proportion of Treg regardless of immunization (Figure 4.8C). T-bet expression was not detected in ex vivo samples. Instead, to assess the induction of $\mathrm{MOG}_{35-55}$-specfic Th1 cells as well as Th17 cells, splenocytes were isolated, cultured and stimulated with $\mathrm{MOG}_{35-55}$-peptide to investigate antigen-specific IFN- $\gamma$ and IL-17A recall responses as a measure of Th1 and Th17 induction, respectively. Immunized mice produced significant levels of IFN- $\gamma$ (Figure 4.8D) and IL-17A (Figure 4.8E) in response to $\mathrm{MOG}_{35-55}$-peptide while unimmunized mice did not, indicating that immunization induced strong antigen-specific responses. Clozapinetreated, immunized mice produced similar levels of IFN- $\gamma$ to vehicle-treated immunized mice indicating no impairment in the antigen-specific induction of Th1 cells (Figure 4.8D). Consistent with the finding that no difference in Th17 cells was detected, no difference in IL17A production was measured between clozapine and vehicle-treated mice (Figure 4.8E) indicating that clozapine did not affect the induction of $\mathrm{MOG}_{35-55}$-peptide specific $\mathrm{T}$ cell responses at this time point. 


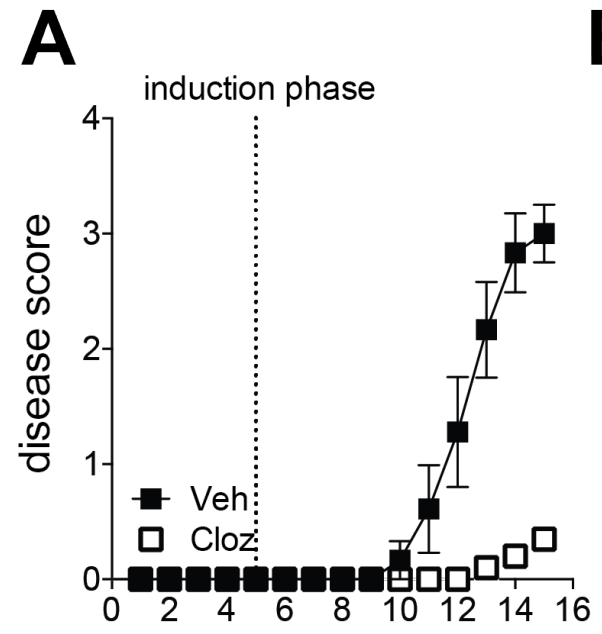

days post immunization

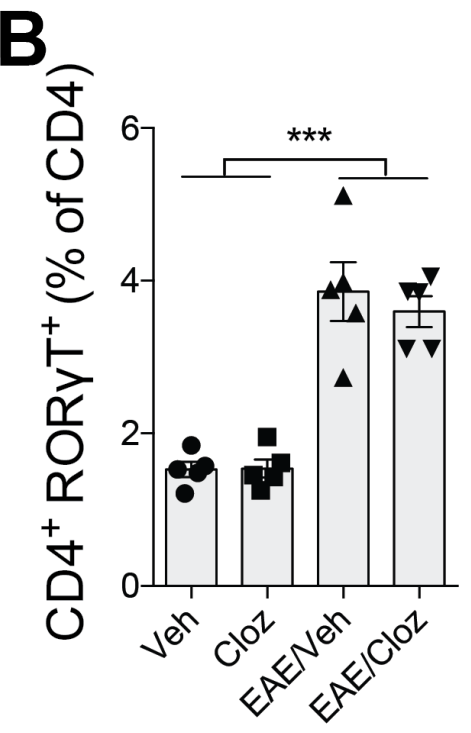

\section{C}
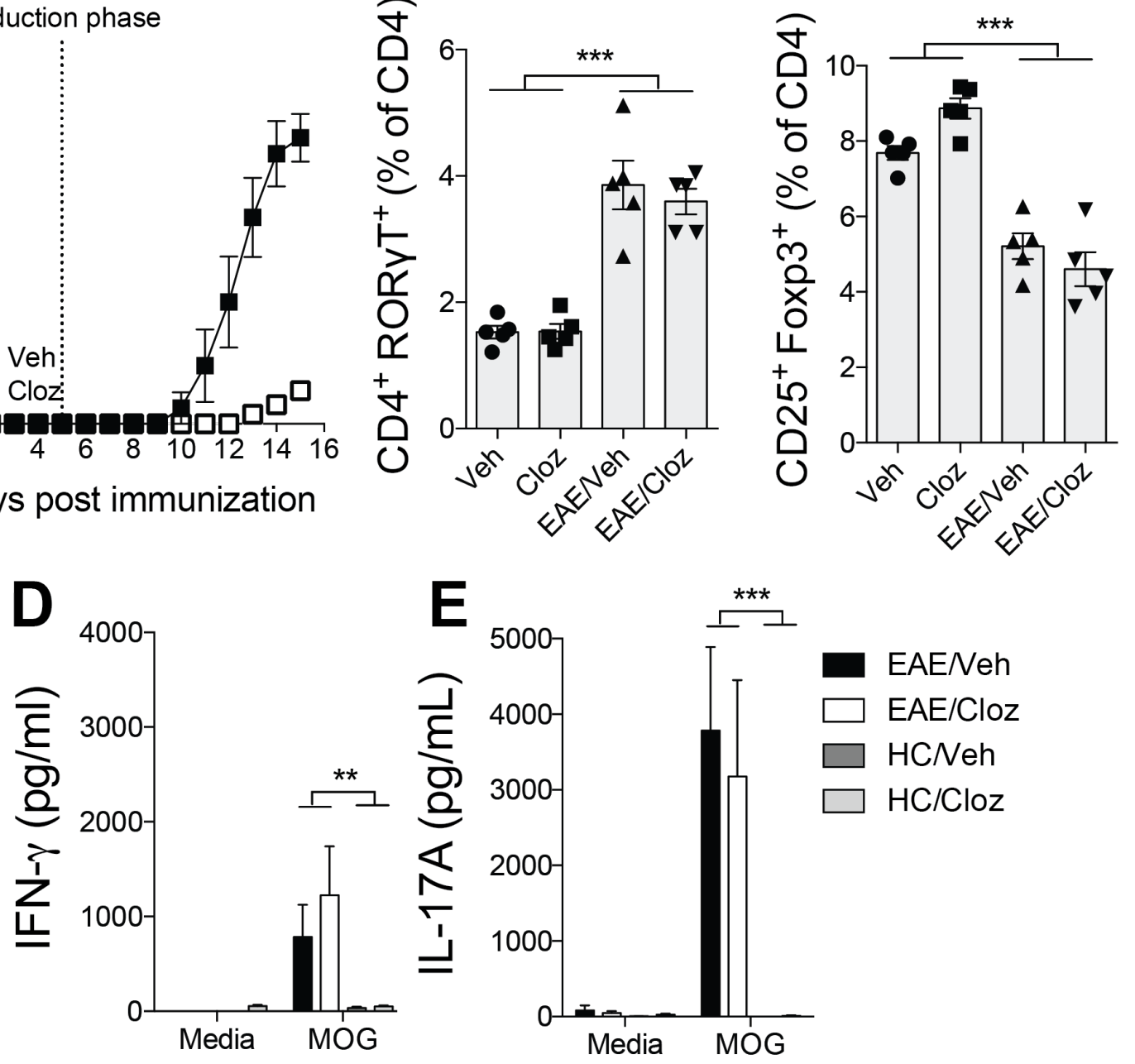

Figure 4.8 Clozapine had no effect on Th development during the induction phase of EAE

A-E Mice were treated with clozapine $(60 \mathrm{mg} / \mathrm{kg} / \mathrm{day})$ or vehicle in their drinking water one day prior to EAE induction. Spleens were analysed for Th17 and Treg cells at 5 d.p.i (induction phase) A Disease score of mice with line at day 5 indicating time of euthanasia. Th17 cells (B) and Treg (C) as percent of CD4 in spleen 5 d.p.i. Rationale for gating ROR $\gamma \mathrm{T}^{+}$and Foxp3 ${ }^{+} \mathrm{CD} 4 \mathrm{~T}$ cells are presented in Appendix II. D-E IFN- $\gamma(\mathbf{D})$ and IL-17A (E) in the supernatant after 72 hours ex-vivo re-stimulation of splenocytes with MOG $_{35-55}$ peptide). Shown are means and SEM of individual mice from one experiment $(n=5$ mice/group).

By the effector phase of EAE 15 d.p.i, all vehicle-treated mice reached peak EAE disease whereas clozapine-treated mice had only mild or no EAE (disease onset is approx. 16 d.p.i) (Figure 4.9A). To investigate whether this remarkable discordance in disease course was associated with defective induction of Th1, Th17 or Treg, splenocytes were isolated 15 d.p.i and analysed by flow cytometry. As in the induction phase, clozapine treatment did not affect the proportion of Th17 cells when compared to vehicle-treated mice despite the fact that EAE significantly increased Th17 cells in the spleen (Figure 4.9B). In contrast to the induction 
phase, Treg proportions in the spleen increased significantly in immunized mice 15 d.p.i., but no detectable differences were observed between clozapine- and vehicle-treated mice (Figure 4.9C). Finally, to assess induction of $\mathrm{MOG}_{35-55}$-specific recall responses 15 d.p.i, splenocytes were isolated and re-stimulated with $\mathrm{MOG}_{35-55}$-peptide and assessed for IFN- $\gamma$ and IL-17A production. As expected, immunized mice produced significant levels of IFN- $\gamma$ (Figure 4.9D) and IL-17A (Figure 1.8E) while unimmunized mice did not, indicating induction of $\mathrm{MOG}_{35}$ 55-peptide specific responses to EAE induction. Similar to the induction phase, clozapinetreated mice did not have impaired induction of IFN- $\gamma$ (Figure 4.9D) or IL-17A (Figure 4.9E) in response to $\mathrm{MOG}_{35-55}$-peptide indicating that clozapine treatment does not alter the induction of antigen-specific $\mathrm{T}$ cell responses. It should be noted that at 15 d.p.i, Th1 responses dominated over Th17 responses, in contrast to the induction phase where Th17 responses dominated over Th1, consistent with a previously published study (Yoshifumi Sonobe et al., 2007). To support the finding that clozapine did not alter differentiation of Th1 and Th17, intracellular cytokine staining was performed on splenocytes to determine the proportion of IFN- $\gamma$ and IL-17A producing CD4 T cells in mice treated with clozapine or vehicle (Figure 4.9F). No difference in IFN- $\gamma^{+}$cells (Figure 4.9G) or IL-17A ${ }^{+}$(Figure 4.9H) CD4 T cells was detected between vehicle and clozapine treated mice. Altogether, these results show that although clozapine-treated mice were protected from disease at 15 d.p.i, protection from EAE was not associated with defective $\mathrm{MOG}_{35-55}$-peptide specific CD4 $\mathrm{T}$ cell responses during the induction or the effector phases of EAE. 
A $\quad$ B $\quad$ C
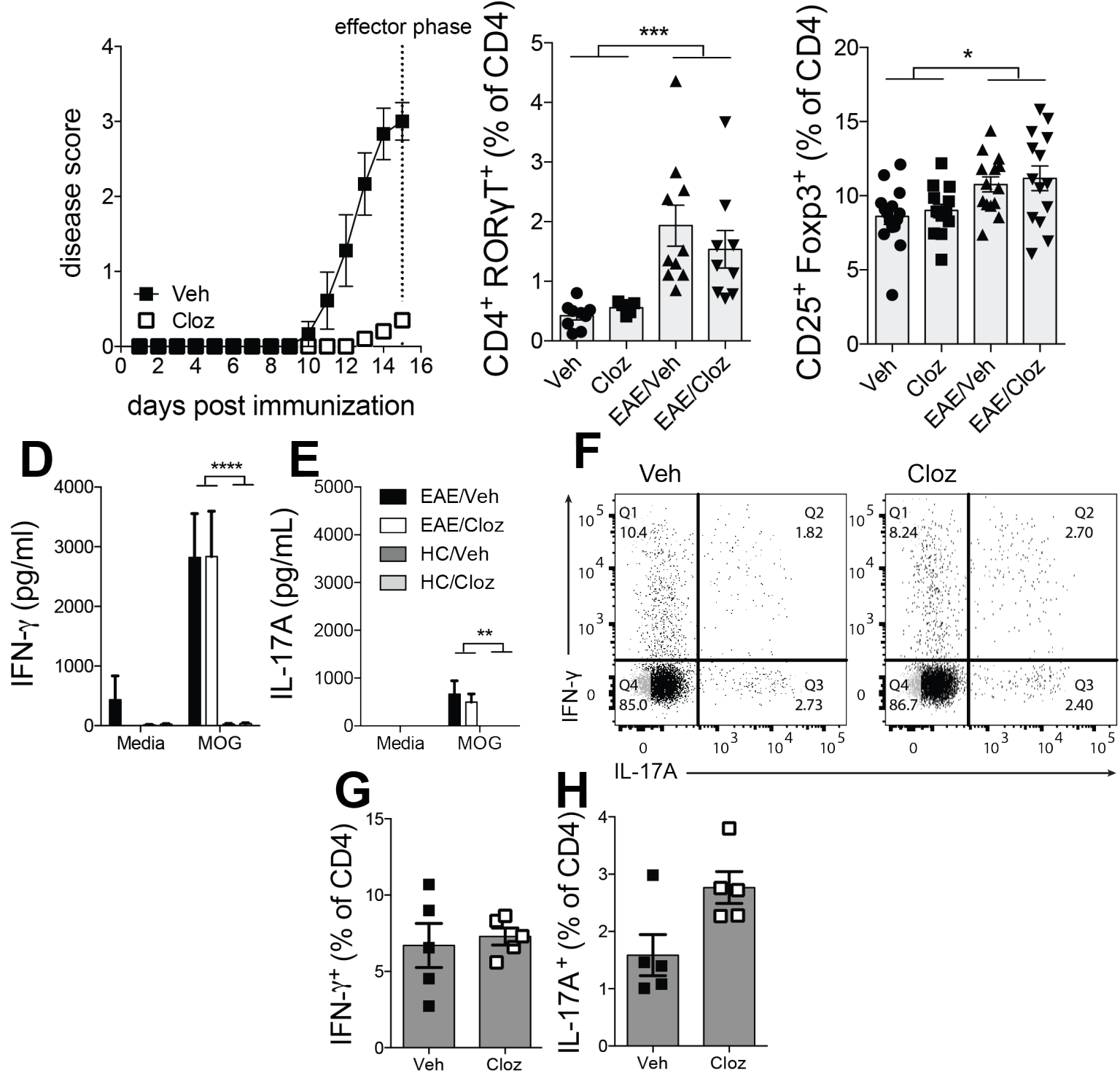

\section{Figure 4.9 Clozapine did not alter Th subset development during the effector phase of EAE}

A-H Mice were treated with clozapine $(60 \mathrm{mg} / \mathrm{kg} / \mathrm{day})$ or vehicle in their drinking water one day prior to EAE induction. Spleens were analysed for Th17 and Treg cells at 15 d.p.i (effector phase) A Disease score of mice with line at day 15 indicating time of euthanasia. Th17 cells $(\mathbf{B})$ and Treg $(\mathbf{C})$ as percent of CD4 in spleen 15 d.p.i. Rationale for gating ROR $\gamma \mathrm{T}^{+}$and Foxp3 ${ }^{+} \mathrm{CD} 4 \mathrm{~T}$ cells are presented in Appendix II. D-E IFN- $\gamma$ (D) and IL-17A (E) in the supernatant after 72 hours ex-vivo re-stimulation of splenocytes with $\mathrm{MOG}_{35-55}$ peptide). F Representative dot plots of intracellular cytokine staining for IFN- $\gamma^{+}$and IL-17A ${ }^{+}$CD4 T cells ex vivo. Rationale for placing quadrant gates are shown in Appendix II. G-H G IFN- $\gamma^{+}$and $\mathbf{H}$ IL-17A ${ }^{+}$CD4 T cells presented as percentage of CD4. Shown are means and SEM of individual mice from two experiments combined ( $\mathrm{n}=$ combined $10 \mathrm{mice} /$ group; A-C), one experiment representing three $(\mathrm{n}=5$ mice/group; D-E) and one experiment ( $\mathrm{n}=5$ mice/group; F-H). For statistical analyses, a two-way ANOVA (B-E) or Student's t-test (G-H) was used. * p $<0.05, * * *<0.001$. 


\subsubsection{Clozapine did not alter $M O_{35-55}$-specific $T$ cell expansion after EAE induction}

Since the effect of clozapine treatment on the proliferation of $\mathrm{MOG}_{35-55}$-specific $\mathrm{T}$ cells is currently unknown, it was necessary to determine whether clozapine significantly affected the proliferation of myelin reactive $\mathrm{CD} 4^{+} \mathrm{T}$ cells as a potential mechanism by which clozapine could reduce or delay EAE disease. An in vivo proliferation assay was performed by transferring CFSE labelled cells from 2D2 $2^{\mathrm{MOG} 35-55} \mathrm{TCR}$ mice expressing CD45.2 into recipient congenic B6-SJ ${ }^{\mathrm{ptprca}}$ mice expressing CD45.1. The use of congenic mouse strains allowed for the discrimination of donor $\mathrm{CD} 4^{+} 2 \mathrm{D} 2^{\mathrm{MOG} 35-55}$ from host (Figure 4.10A). Recipient mice were then immunized to induce EAE and the proliferation of transferred $2 \mathrm{D} 2^{\mathrm{MOG} 35-55} \mathrm{TCR} \mathrm{CD} 4^{+} \mathrm{T}$ cells was measured after 5 days by CFSE dilution. 2D2 $2^{\text {MOG35-55 }}$ TCR CD4 $4^{+}$proliferated in response to immunization with $\mathrm{MOG}_{35-55}$-peptide in vehicle-treated mice. Interestingly, treatment with clozapine using a regime previously shown to be effective at reducing EAE disease did not affect the percentage of cells that proliferated nor the proliferation index of these cells in the draining lymph nodes (Figure 4.10B), spleen (Figure 4.10C) and peripheral blood (Figure 4.10D), indicating that although clozapine effectively delayed disease onset and disease severity, treatment did not alter the proliferative capacity of $\mathrm{MOG}_{35-55}$-peptide-specific CD4 T cells during EAE induction. 


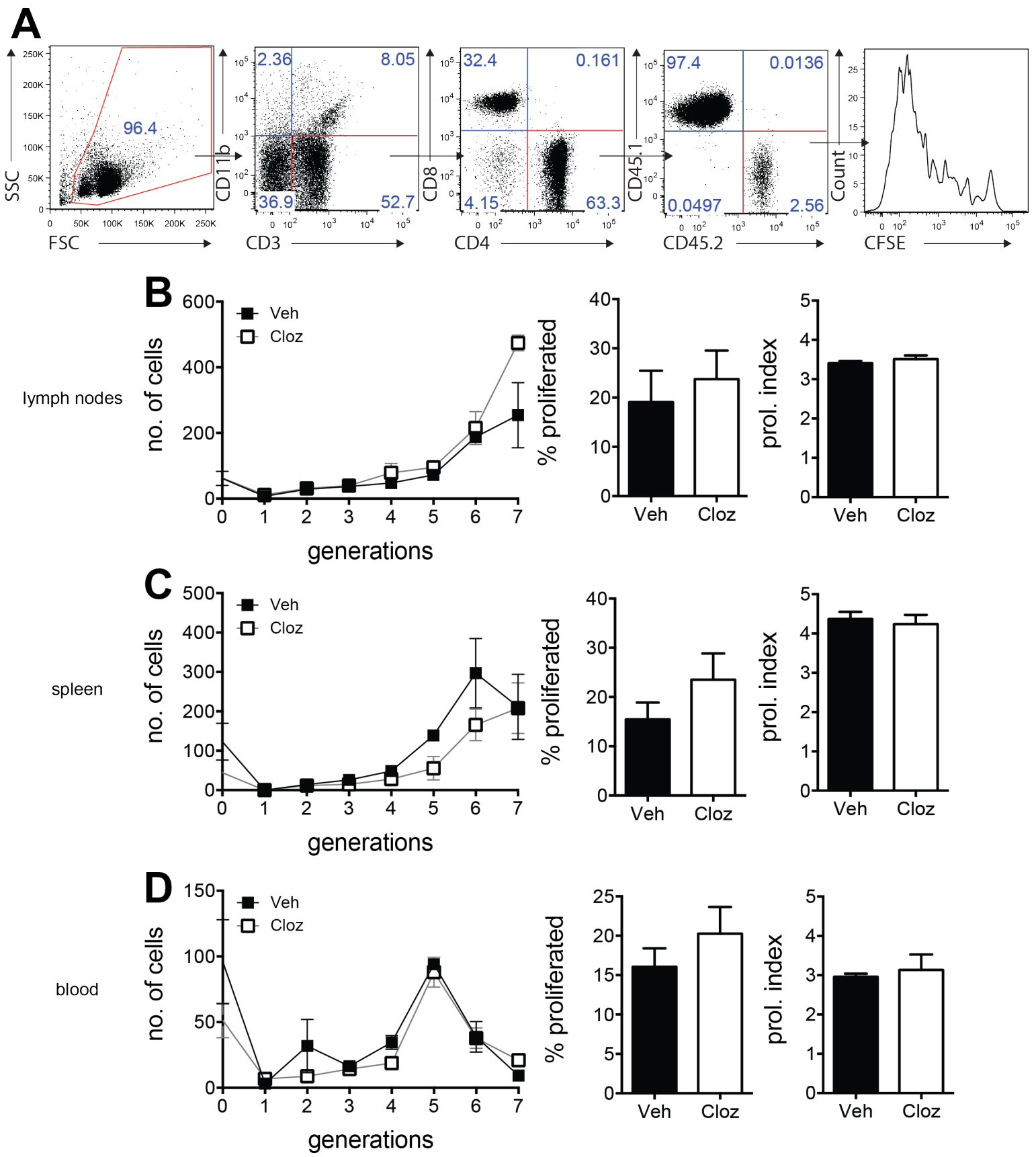

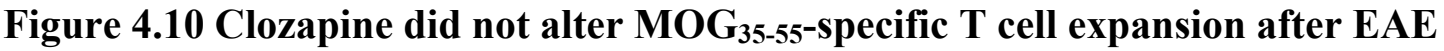
induction

A-D 20x10 $0^{6}$ CFSE labelled cells from 2D2 $2^{\text {MOG35-55 }}$ TCR (CD45.2) mice were injected i.p into congenic B6$\mathrm{Sj}^{\text {ptprca }}$ (CD45.1) mice one day prior to immunization for EAE. After 5 days, mice were euthanized. Representative gating strategy for identifying $2 \mathrm{D} 2^{\mathrm{MOG} 35-55} \mathrm{TCR} C D 4^{+} \mathrm{T}$ cells in draining lymph nodes. First, single cells were identified using FSC-A vs FSC-H and followed by cells of interest using FSC-A and SSC-A. Lymphocytes were identified by $\mathrm{CD} 11 \mathrm{~b}^{-} \mathrm{CD} 3^{+}$followed by $\mathrm{CD} 8^{-} \mathrm{CD} 4^{+}$to identify CD4 T cells. Donor cells were then identified by CD $45.1^{-} \mathrm{CD} 45.2^{+}$expression and analysed using FlowJo proliferation platform (A). Shown are graphs of cells in each generation assessed by CFSE dilution, \% of cells proliferated and proliferation index (no. of divisions/no. of cells that divided) (left to right) from draining lymph nodes (B; mesenteric and inguinal lymph nodes), spleen (C) and blood (D). Shown are means and SEM of values from individual mice $($ Veh $n=2$, Cloz $n=3$ ) from one representative experiment of two. 


\subsubsection{The clozapine-mediated delay and reduction in EAE was not mediated by Treg}

Previously published studies have described the important role of Treg for limiting disease severity and controlling effector function of T cells during EAE (Koutrolos, Berer, Kawakami, Wekerle, \& Krishnamoorthy, 2014; McGeachy, Stephens, \& Anderton, 2005; Montero et al., 2004; X. Zhang et al., 2004). Indeed, we have shown that the Treg population increases significantly at the effector phase of EAE before entering the recovery phase. In this study, we found that clozapine promotes the differentiation of Treg and also increases the expression of Foxp3 in an in vitro model of $\mathrm{T}$ cell differentiation. The Foxp3 transcription factor is the master regulator of Treg suppressive function and is absolutely essential for the identification of Treg. The significance of Foxp3 expression in Treg has been reported to be more informative than Treg frequency for suppressive function when assessing longevity in an allograft transplant model, suggesting that an increase in Foxp3 expression may also improve the suppressive function of Treg in EAE. Given that clozapine is able to increase Foxp3 expression in Treg in an in vitro model, it was essential to assess whether protection from EAE by clozapine treatment was facilitated by enhancing regulatory $\mathrm{T}$ cell function in vivo.

To achieve this goal experimentally, Treg were neutralized using an anti-CD25 mAb (PC61; $200 \mu \mathrm{g} /$ mouse) injected prior to the induction of EAE and maintained at regular intervals to maintain robust CD25 neutralization throughout the course of the experiment (Figure 4.11A). This approach has been used in multiple EAE models to effectively block CD25 dependent Treg function in vivo (Akirav, Bergman, Hill, \& Ruddle, 2009; McGeachy et al., 2005; Montero et al., 2004; Leigh A. Stephens, Gray, \& Anderton, 2005). Firstly, to assess CD25 neutralization, an Alexa Fluor 488 conjugated anti-CD25 (PC61) was used to detect CD25 molecules unbound by purified anti-CD25 on $\mathrm{CD} 4^{+} \mathrm{Foxp}^{+} \mathrm{T}$ cells. No CD25 binding was detected at 0 (Figure 4.11B), 10 (Figure 4.11C) and 17 d.p.i (Figure 4.11D) demonstrating complete and effective neutralization of Treg during the course of the experiment. 
A
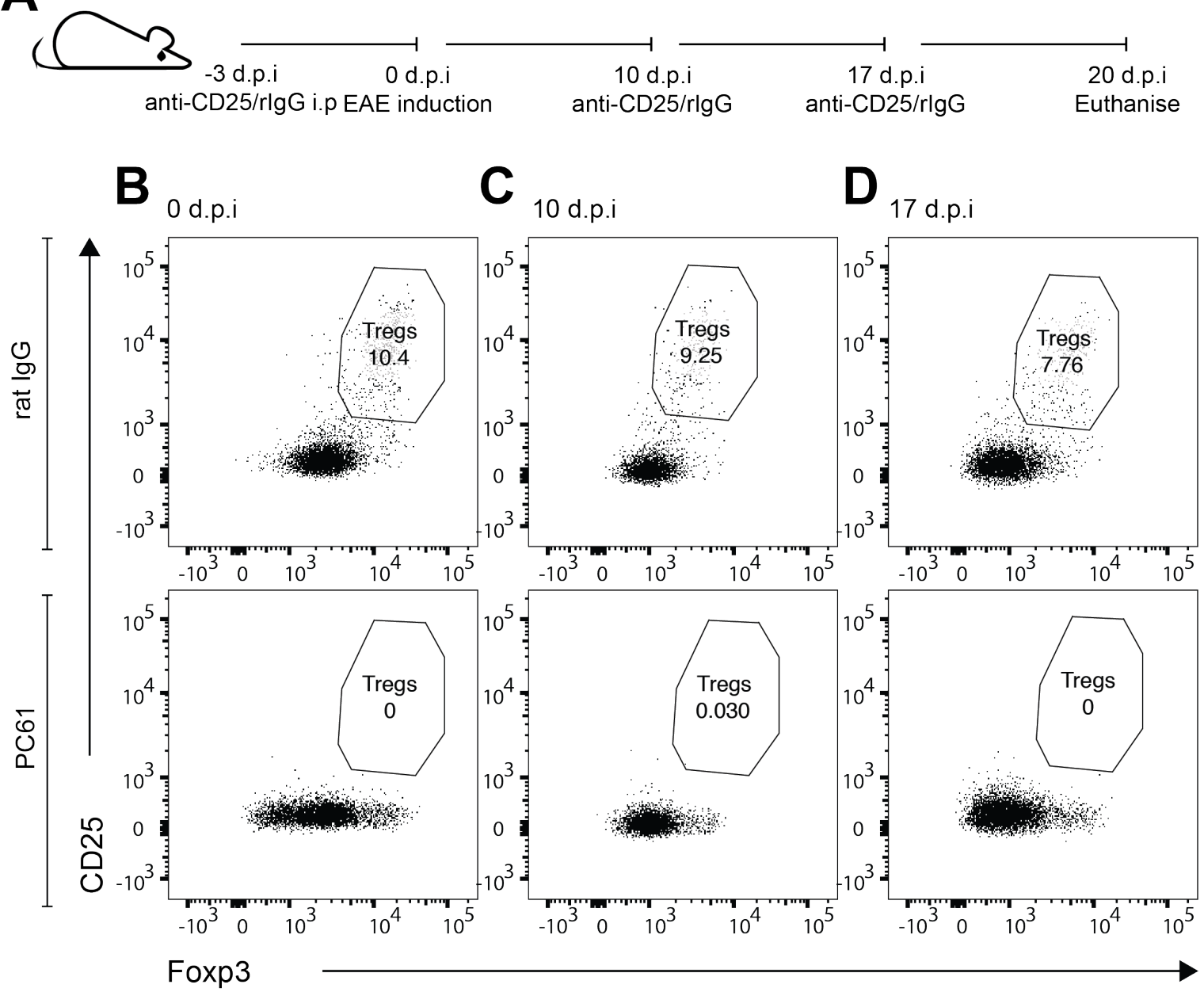

Figure 4.11 Schematic for Treg neutralization

A-D Treg neutralization. Timeline of anti-CD25 injections and EAE induction (A). Analysis of splenocytes for anti-CD25 conjugated to Alexa Fluor 488 after injection with either purified rat IgG or purified anti-CD25 (PC61) from mice euthanized at $0(\mathbf{B}), 10(\mathbf{C})$ and 17 d.p.i (D). Shown are representative flow cytometry plots at each time point from one experiment representing two.

When Treg were neutralized by anti-CD25 EAE disease was exacerbated in vehicle treated mice (Figure 4.12A). Although the onset of disease is not affected by anti-CD25 (Figure 4.12B), vehicle treated EAE mice that received anti-CD25 injections reached a significantly higher peak disease score (Figure 4.12C) whereas mice that received clozapine maintained robust protection from EAE and had a similar onset of disease (Figure 4.12B), peak disease score (Figure 4.12C) and overall disease burden (Figure 4.12D) to mice that received rat $\operatorname{IgG}$ control antibody. Antigen-specific recall responses to $\mathrm{MOG}_{35-55}$-peptide were measured to 
further determine whether clozapine treatment had affected Treg function in controlling antigen-specific responses. Splenocytes were re-stimulated with $\mathrm{MOG}_{35-55}$-peptide and IFN- $\gamma$ and IL-17A produced was measured to assess Th1 and Th17 effector response at 20 d.p.i. CD25-neutralized mice appeared to produce more IFN- $\gamma$ (Figure 4.12E) and IL-17A (Figure 4.12F) than mice that received rat IgG control, although this did not reach statistical significance. Nevertheless, no difference in $\mathrm{MOG}_{35-55}$-peptide specific IFN- $\gamma$ or IL-17A were observed between clozapine and vehicle treated mice irrespective of their CD25 neutralization status. These results indicate that while Treg function was required for limiting disease severity in EAE, their function was dispensable for the protective effect of clozapine. This finding suggests that although clozapine may enhance Treg function in vitro, their function was not responsible for delaying and reducing disease during EAE. 

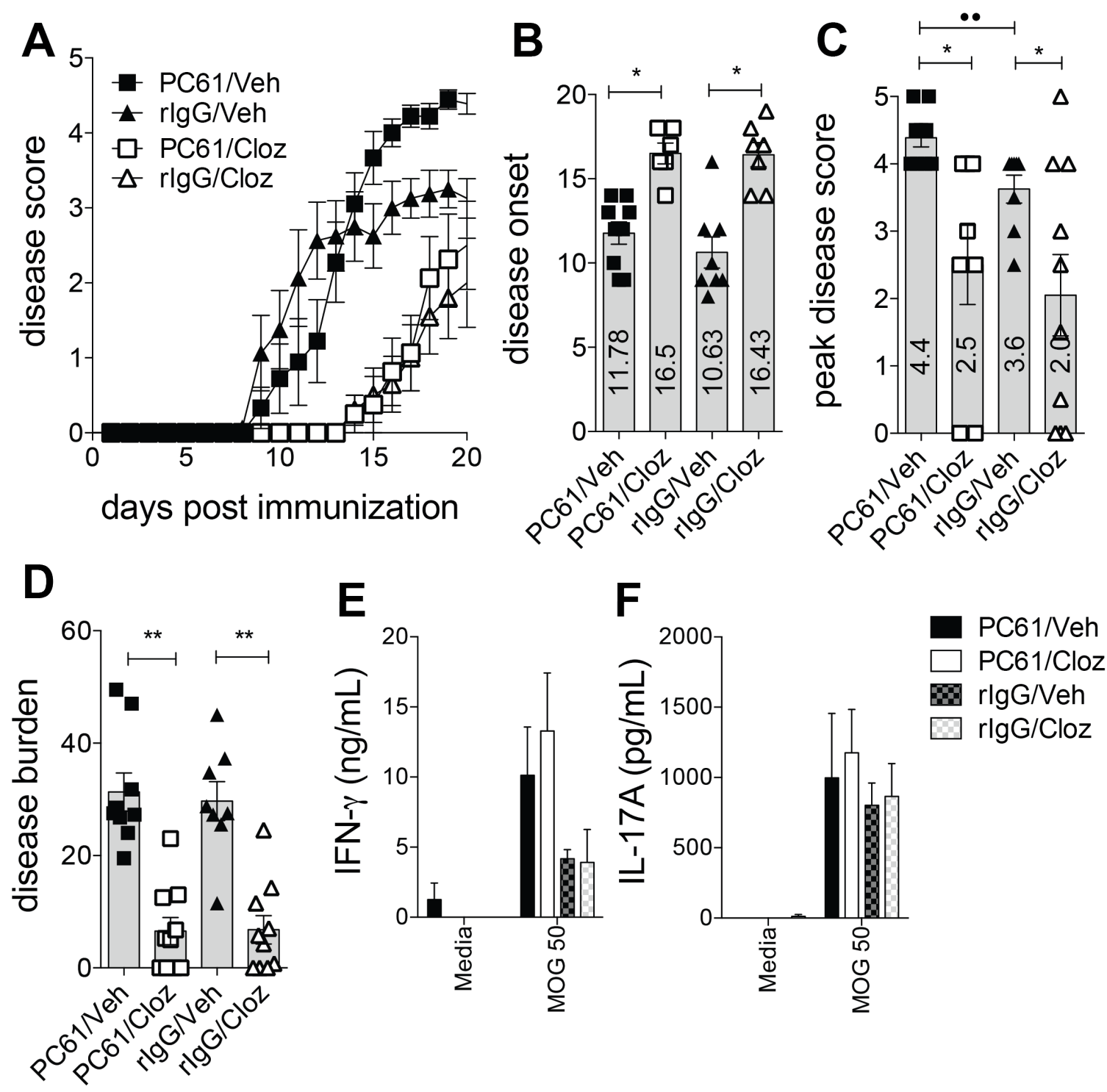

Figure 4.12 Protection by clozapine was not dependent on $\mathrm{CD}^{+} 5^{+}$regulatory $\mathrm{T}$ cells

A-F Treg were neutralized in mice 3 days before EAE induction and maintained throughout the experiment. Clozapine or vehicle was added to drinking water one day before induction for EAE and mice scored daily for disease (0; normal - 5; moribund). Disease score of mice up to 20 d.p.i. (A). First onset of disease of mice that succumbed to EAE (B). Peak disease score reached $(\mathbf{C})$. Disease burden assessed by area under the curve analysis (D). Shown are the means and SEM of individual mice from two experiments combined (combined $n$ $>7$ mice in each group). E-F IFN- $\gamma(\mathbf{E})$ and IL-17A (F) production in the supernatant after MOG $_{35-55}$-peptide specific recall for 72 hours. Shown are the means and SEM of individual mice from one experiment. $n>4$ mice in each group. $* \mathrm{p}<0.05, * * \mathrm{p}<0.01$ by two-way ANOVA and Sidak's multiple comparison test. $\bullet \bullet p<0.01$ by Mann-Whitney. 


\subsubsection{Clozapine did not alter the encephalitogenic capacity of myelin-specific $\mathbf{T}$ cells}

The experiments conducted in this study have indicated that clozapine can affect the differentiation of CD4 T cells into Th1, Th17 and Treg subsets. Although these changes were not observed during EAE, these results indicate that the presence of clozapine during $\mathrm{T}$ cell activation could have the potential to affect downstream effector function and impair the capacity to induce EAE. To address whether clozapine has a critical effect on the functional capacity of myelin-specific $T$ cells to induce EAE, a passive transfer of EAE model was developed where $\mathrm{MOG}_{35-55}$-peptide reactive cells were isolated from immunized donor mice, activated to become encephalitogenic in vitro and then transferred into naïve recipients. Clozapine was added during the activation stage to determine whether it affected the capacity of the $\mathrm{T}$ cells to induce EAE.

First, an effective adoptive transfer model of EAE was developed. Adoptive transfer of cells activated with $\mathrm{MOG}_{35-55}$-peptide and IL-2 resulted in mild EAE in only a small fraction of mice (Figure 4.13). In contrast, when IL-12p70 and anti-IFN- $\gamma$ were added, adoptive transfer reliably induced severe EAE (Figure 4.13). The fact that adoptive transfer of $\mathrm{MOG}_{35-55^{-}}$ specific $\mathrm{T}$ cells stimulated with $\mathrm{MOG}_{35-55}$-peptide and IL-2 induced only mild EAE with low incidence while adoptive transfer of myelin-specific CD4 T cells expanded with $\mathrm{MOG}_{35-55^{-}}$ peptide, IL-12p70 and anti IFN- $\gamma$ induced severe disease, highlights the importance of the cytokine milieu during activation for encephalitogenicity. 


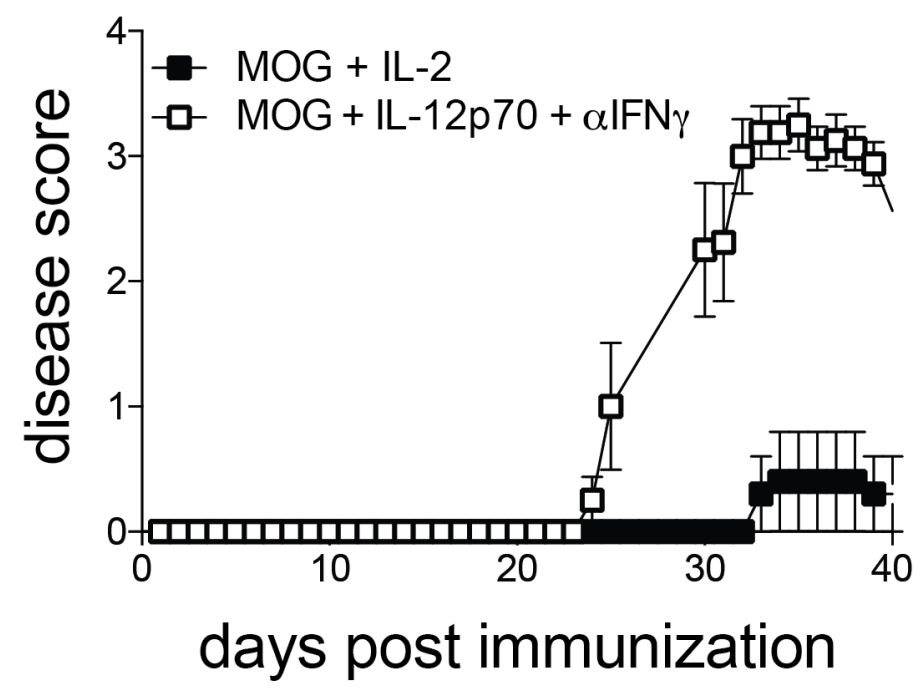

Figure 4.13 Adoptive transfer of $T$ cells expanded in MOG35-55-peptide with IL12 p70 and anti-IFN- $\gamma$ was sufficient to induce $E A E$

Donor mice are immunized with $\mathrm{MOG}_{35-55}$-peptide and euthanied 12 d.p.i. Donor cells from spleen and lymph nodes were harvestd 12 d.p.i and stimulated with $\mathrm{MOG}_{35-55}$-peptide and IL-2 (10 U/mL) (closed box) or $\mathrm{MOG}_{35}$ 55-peptide and differentiated with IL-12p70 $(20 \mathrm{ng} / \mathrm{mL})$ and anti-IFN- $\gamma(10 \mu \mathrm{g} / \mathrm{mL})$ (open box) for 96 hours. $2 \times 10^{7}$ cells were adoptively transferred to naïve recipients to induce passive EAE. Mice were weighed and scored daily for disease $(n=10$ mice in each group from one experiment).

To investigate whether clozapine could affect downstream effector function, $\mathrm{MOG}_{35-55^{-}}$ specific CD4 T cells were activated and expanded in the presence of clozapine before adoptive transfer into naïve mice. Adoptive transfer of encephalitogenic cells induced EAE whether they were activated in the presence of clozapine or vehicle with a similar disease course (Figure 4.14A) and weight change (Figure 4.14B). Clozapine did not alter overall disease burden (Figure 4.14C), suggesting that the presence of clozapine during the activation of CD4 T cells did not alter their function in a way that could affect their potential to induce EAE disease in recipient mice. 

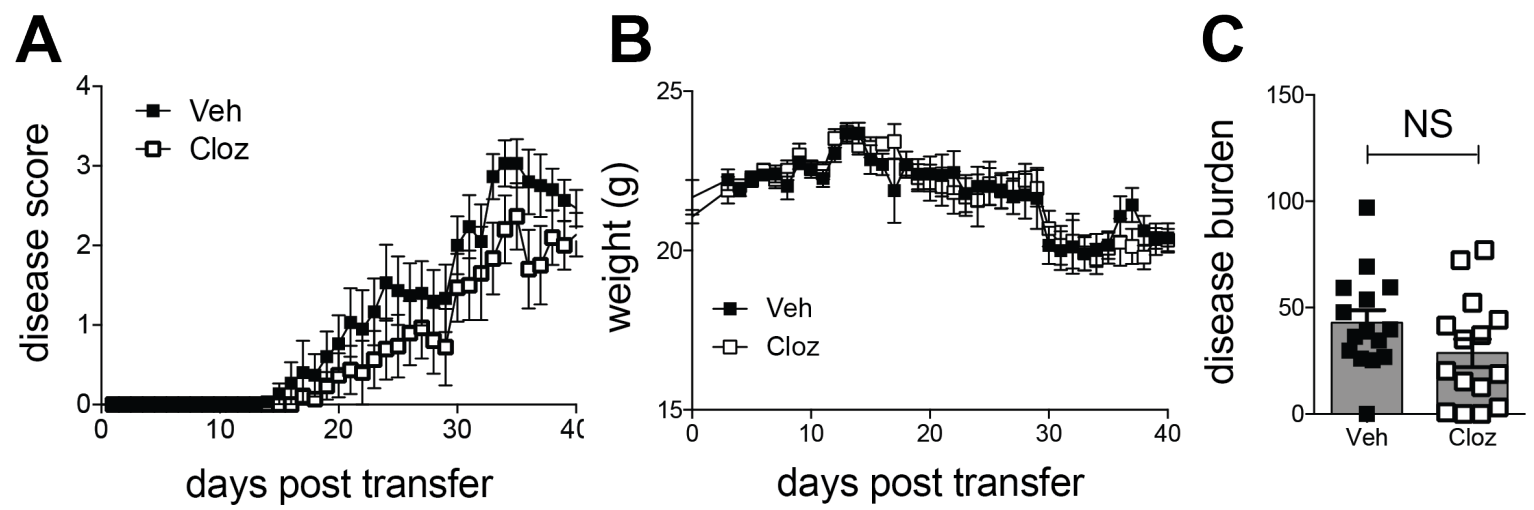

\title{
Figure 4.14 Clozapine did not alter encephalitogenicity of $M_{0 G} \mathbf{O G}_{35-55}$-specific $\mathbf{T}$ cells
}

\begin{abstract}
A-C Spleens and draining lymph nodes were harvested from immunized donor mice and expanded in vitro with $\mathrm{MOG}_{35-55}$ peptide, IL-12p70 $(20 \mathrm{ng} / \mathrm{mL})$ and anti-IFN- $\gamma(10 \mu \mathrm{g} / \mathrm{mL})$ in the presence of clozapine $(20 \mu \mathrm{M})$ or vehicle for 96 hours. $20 \times 10^{6}$ cells were then injected i.p into recipient mice to induce passive EAE. Mice were weighed and scored daily for disease (0; normal - 5; moribund). A Disease score of mice up to 40 d.p.i B Weights of mice up to 40 d.p.i. C Disease burden of mice assessed by area under the curve analysis. Shown are the means and SEM of individual mice from 3 independent experiments combined. (combined $n=15$ mice in each group). Mann-Whitney test was used to determine whether disease burden was statistically different between vehicle and clozapine.
\end{abstract}

\subsubsection{Clozapine did not protect from adoptively transferred EAE disease}

The adoptive transfer of EAE model previously described has been instrumental in delineating the role of individual components and immunological events that occur during EAE. This chapter has already shown that encephalitogenic $\mathrm{T}$ cells can be manipulated before transfer into mice to investigate the effects of individual cytokines or compounds on EAE. By adoptively transferring encephalitogenic $\mathrm{T}$ cells into naïve mice, the induction phase of EAE can effectively be omitted, allowing simplified analysis of a treatment regimen such as clozapine to be active only during the effector phase of disease and not in the induction phase. To address whether clozapine protects from EAE by altering disease mechanisms during the induction or the effector phase of disease, passive EAE was induced in mice either treated with clozapine or vehicle. As expected, vehicle treated mice succumbed to adoptively transferred EAE. However, somewhat unexpectedly, mice treated with clozapine were no longer protected from EAE when induced by adoptive transfer of encephalitogenic T cells (Figure 4.15A). Mice which were treated with clozapine had a similar disease onset (Figure 4.15B) and disease burden to untreated mice (Figure 4.15C). These results show that although clozapine was effective at delaying EAE onset and reducing disease severity when EAE was induced actively, clozapine was not protective when EAE was induced using adoptively transferred 
encephalitogenic $\mathrm{T}$ cells generated in this way. These results could indicate that clozapine has an important effect on the induction phase of disease, but is not similarly effective during the effector phase. Alternatively, the protocol used to induce passive EAE may be so effective at inducing EAE disease that the effect of clozapine is no longer detectable.
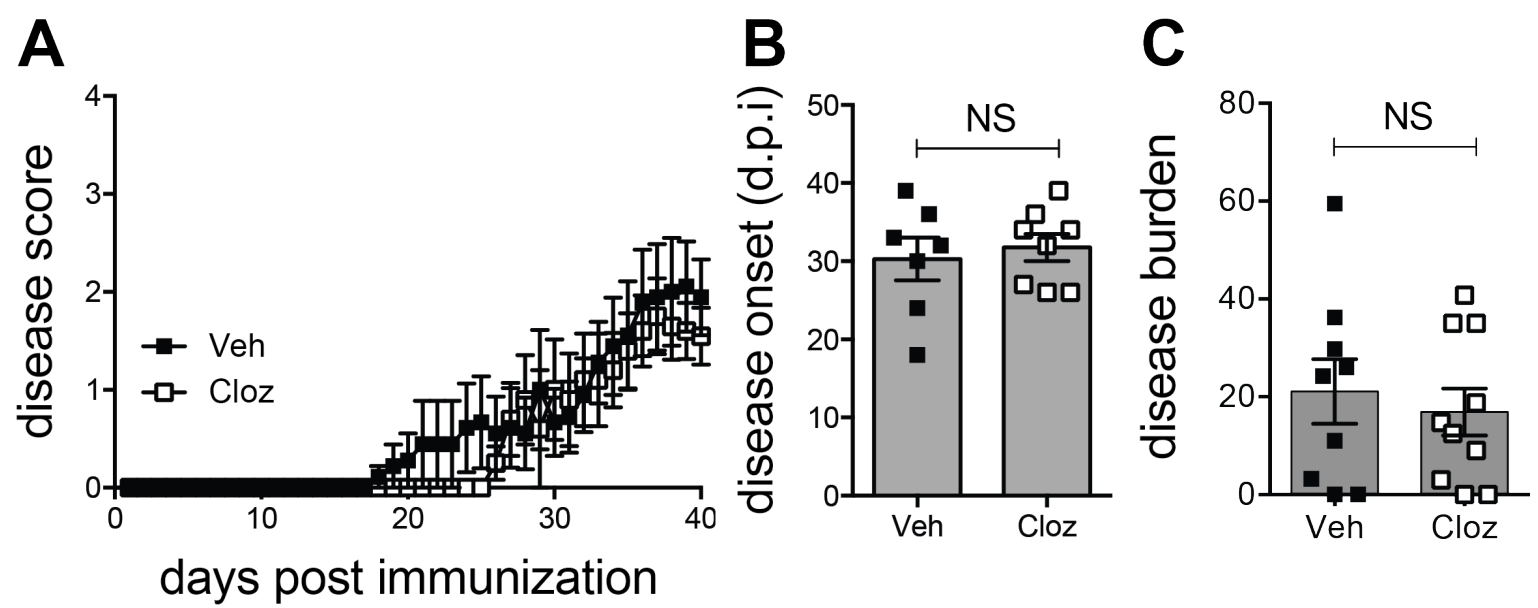

\section{Figure 4.15 Clozapine did not protect from adoptively transferred EAE disease}

A-C Spleens and draining lymph nodes were harvested from immunized donor mice and expanded in vitro with $\mathrm{MOG}_{35-55}$ peptide, IL-12p70 $(20 \mathrm{ng} / \mathrm{mL})$ and anti-IFN- $\gamma(10 \mu \mathrm{g} / \mathrm{mL})$ for 96 hours. $2 \times 10^{7}$ cells were then injected i.p into recipient mice to induce passive EAE one day after adding clozapine $(60 \mathrm{mg} / \mathrm{kg} / \mathrm{day})$ or vehicle to the drinking water. Mice were weighed and scored daily for disease (0; normal - 5; moribund). A Disease score of mice up to 40 d.p.i. B Disease onset of mice that got sick (d.p.i; days post immunization) C Disease burden of mice assessed by area under the curve analysis. Shown are the means and SEM of individual mice from 3 independent experiments combined. (combined $n=15$ mice in each group). Mann-Whitney test was used to determine whether disease burden was statistically different between vehicle and clozapine. 


\subsection{Discussion}

Multiple sclerosis is considered an autoimmune disease mediated by self-reactive $\mathrm{T}$ cells with many studies suggesting that self-reactivity to myelin antigens is central to disease. In support of this is the EAE model, which is induced by immunization with the self-peptide MOG and results in the formation of lesions similar to those found during multiple sclerosis. We have shown that atypical antipsychotic agents like clozapine and risperidone are effective at reducing disease in EAE with potential to be effective in multiple sclerosis. Previous reports have indicated that clozapine may be able to alter intracellular signalling which theoretically has the potential to alter signalling of $\mathrm{T}$ cells during activation and differentiation. This chapter investigated the effects of clozapine treatment on various CD4 T cell functions as a potential mechanism by which clozapine may reduce or delay EAE disease. It was found that although clozapine effectively reduced disease severity and delayed disease onset, the proliferative capacity of $\mathrm{MOG}_{35-55}$-specific CD4 $\mathrm{T}$ cells and their differentiation into Th1 Th17 or Treg subsets after immunization were unaffected. Clozapine-treated mice maintained robust antigen-specific responses that were comparable to untreated mice despite having significantly attenuated disease. In vitro, clozapine promoted Treg differentiation but in vivo Treg were not involved in disease protection. These results show that protection from EAE is not associated with defective antigen-specific responses in the periphery and indicate a potential mechanism of protection that involves other mechanisms, tissues and immune cell types.

During the induction phase of EAE, $\mathrm{MOG}_{35-55}$ in complete Freund's adjuvant is presented by activated dendritic cells to cognate CD4 $\mathrm{T}$ cells which then respond by proliferating and differentiating into effector subsets for immediate response. Modifying the proliferation of CD4 $\mathrm{T}$ cells can effectively suppress EAE disease; for example, the clinically approved treatment for multiple sclerosis, Mitoxantrone, works in this way by disrupting DNA synthesis and preventing proliferation (Neuhaus et al., 2005). Similarly, microtubule-stabilizing agents can impair $\mathrm{MOG}_{3-55}$-peptide specific T cell proliferation (David O'Sullivan et al., 2013). It is therefore sensible to question whether clozapine delays the onset and attenuates disease in EAE by suppressing the proliferation of self-reactive $\mathrm{T}$ cells. These experiments provide evidence that clozapine does not alter the proliferation of $\mathrm{MOG}_{35-55}$-peptide specific CD4 $\mathrm{T}$ cells in the periphery after immunization. Future studies need to be performed to determine whether $\mathrm{T}$ cell re-activation in the CNS tissue is affected by clozapine treatment. 
Given the relatively recent discovery that atypical antipsychotic agents can modify the immune response, published experimental articles that describe precise mechanisms of action on immune cells remain scarce. There is however a number of studies describing the effect of clozapine on cell signalling pathways in other cell types using tissue culture experiments, and these experiments show that clozapine in cell culture can inhibit the phosphorylation of Akt and modulate GSK-3 $\beta$ in response to a stimulus like epidermal growth factor (Shin et al., 2006). However, studies have also shown that clozapine may increase Akt phosphorylation (Fabio Panariello et al., 2012). In either case, it is known that the Akt pathway is important for metabolism, protein synthesis and during TCR activation. Antigen-specific activation of Akt in T cells activates the mammalian target of rapamycin (mTOR) and GSK-3 $\beta$ downstream of Akt, which has a regulatory role in cell metabolism, growth and differentiation. Firstly, mTOR has a differential influence on the differentiation of CD4 T cells into Th1, Th2, Th17 and Treg cells. Inhibiting mTOR using rapamycin promotes the generation of Foxp $3^{+}$Treg cells (Kopf, de la Rosa, Howard, \& Chen, 2007) and deletion of mTOR in T cells using Cre recombinase system in mice enhances Treg differentiation but not Th1, Th2 or Th17 cells (Delgoffe et al., 2009). These studies highlight the importance translating environmental signals into an appropriate Th1, Th17 or Treg response. It is therefore conceivable to suggest that if clozapine altered intracellular Akt-mTOR signalling, clozapine could differentially affect the differentiation of Th1, Th17 and Treg during EAE.

Downstream of Akt activation is GSK-3 $\beta$ which has already been shown to have a key role in the differentiation of $\mathrm{T}$ cells into distinct $\mathrm{Th}$ subsets as well as considerable evidence that clozapine is able to modulate its activity. The importance of GSK-3 $\beta$ has been demonstrated elegantly in two published articles by Beurel et al. in 2011 and 2013 concluding that when GSK-3 $\beta$ is inhibited using a specific inhibitor, the differentiation of Th17 and Th1 cells are impaired while Treg and Th2 are not affected due to the dependence of Th17 and Th1 cells on active GSK-3 $\beta$ (Beurel et al., 2013; E. Beurel et al., 2011). These studies are supported by published articles from other investigators that demonstrate the dependence of Treg on $\beta$ catenin which is regulated by GSK-3 $\beta$. The pilot experiment presented in this chapter indicated that although iTreg were enhanced by clozapine, no difference in $\beta$-catenin expression was measured. This result may indicate that inhibition of GSK-3 $\beta$ is likely to be not the sole pathway involved but may also involve the mTOR pathway to produce differential effects on $\mathrm{T}$ cell differentiation. However, because of the design of these experiments, it cannot be concluded whether clozapine affected the CD4 T cell to modify Th1, Th17 or Treg 
differentiation directly or by altering the antigen-presenting cells present in cell culture. Still, these experiments provide insight into the effect of clozapine on complex mixed cell interactions to represent more closely those of in vivo immune reactions.

Other cell signalling alterations have also been described in the literature. Clozapine has been shown to activate SMAD3 (Cohen, Sundaresh, \& Levine, 2013), a signalling molecule critical for TGF- $\beta$ signalling and Treg differentiation (Tone et al., 2008). It is therefore possible that clozapine promotes the differentiation of Treg in our model of $\mathrm{T}$ cell differentiation by promoting or enhancing TGF- $\beta$ signalling through activation of SMAD3 and this possibility warrants further investigation. Finally, these findings are consistent with one study by Chen et al. who showed that clozapine inhibits the differentiation of Th1 cells in human peripheral blood mononuclear cells (PBMCs) activated by anti-CD3/CD28 antibodies by suppressing the Akt pathway (M.-L. Chen et al., 2011).

Despite having observed these effects in an in vitro model, clinical benefit was not associated with a defect in the proliferation or differentiation of Th1 or Th17 antigen specific responses in the periphery. Moreover, the fact that Treg were dispensable for EAE protection indicates that clozapine may not be modifying disease primarily by modifying CD4 $\mathrm{T}$ cell responses. However, despite robust antigen-specific peripheral responses, we observed less infiltration of peripheral leukocytes, including T cells in the CNS, raising the possibility that clozapine alters trafficking of immune cells across the BBB. The trafficking of immune cells across the BBB is governed by interactions between a variety of surface cell adhesion molecules on immune cells and endothelial cells. Specifically, the $\alpha 4$-integrin, LFA-1/I-CAM1 and VLA-4/VCAM1 interactions that are dependent on GPCR signalling (Adamson et al., 2002; Chigaev, Waller, Amit, \& Sklar, 2008; Shamri et al., 2002). Since clozapine targets many GPCR (Catapano \& Manji, 2007) and modulates cAMP signalling in the rat brain (Dwivedi, Rizavi, \& Pandey, 2002), it is entirely plausible that clozapine could affect GPCR-dependent trafficking across the BBB. Although this has not yet been investigated, it has previously been shown altering GPCR signalling in brain endothelial cells by pertussis toxin treatment prevents more than $80 \%$ of lymphocyte trafficking (Adamson et al., 2002), suggesting that perturbations in GPCR has potential to alter blood-brain barrier integrity.

The adoptive transfer model of EAE presented in this chapter shows that clozapine does not affect the capacity of $\mathrm{MOG}_{35-55}$ peptide specific $\mathrm{T}$ cells to induce EAE when present during antigen-specific stimulation. This model relied on the immunization of mice with $\mathrm{MOG}_{35-55}$ 
peptide with CFA to expand $\mathrm{MOG}_{35-55}$ specific $\mathrm{T}$ cells before cell culture. In this model, $\mathrm{MOG}_{35-55}$ peptide specific $\mathrm{T}$ cells are antigen experienced and activated before the in vitro restimulation with $\mathrm{MOG}_{35-55}$ peptide and clozapine, potentially limiting the potential effect of clozapine as the CD4 T cells have already undergone transcriptional changes before exposure to clozapine. A possible solution to this is by treating mice in vivo during the induction of $\mathrm{MOG}_{35-55}$ specific $\mathrm{T}$ cells prior to adoptive transfer, however, the significance of this experiment was questionable given that $\mathrm{MOG}_{35-55}$ peptide specific $\mathrm{T}$ cells needed to be restimulated in vitro with $\mathrm{MOG}_{35-55}$ peptide and IL-12p70 to be encephalitogenic before adoptive transfer, during which they would have been removed from the clozapine environment. A potential solution to this challenge is by using naïve $\mathrm{MOG}_{35-55}$ peptide specific CD4 T cells from 2D2 mice instead to avoid the initial immunization step.

Since no impairment in peripheral antigen-specific $\mathrm{T}$ cell responses were detected in clozapinetreated mice, it is hypothesised that clozapine may be acting on immune cell types other than CD4 T cells. One limitation of this hypothesis is that T cell responses within the CNS during clozapine treatment have not yet been investigated and is worthy of further investigation. However, given the previous evidence that clozapine is able to alter the activation of myeloid cells like macrophages and microglia, it is possible that clozapine is able to reduce EAE disease by targeting the inflammatory activation of CNS myeloid cells. This potential is investigated in detail in the following chapters.

\subsection{Conclusions}

These experiments showed that in vitro, clozapine has the potential to impair Th1 and Th17 development while promoting the differentiation of iTreg. However, Treg were not responsible for protection from EAE by clozapine. In addition, the experiments conducted in vivo suggest that there is no associated impairment in peripheral CD4 $\mathrm{T}$ cell function despite effective protection from disease under clozapine treatment. This indicates that clozapine may not primarily be acting on $\mathrm{T}$ cells but instead involve other immune cell types such as infiltrating and resident myeloid cell populations that are predominant in demyelinating lesions. 
Chapter 5: The effect of clozapine on the activation of myeloid cells 


\subsection{Introduction}

The central nervous system (CNS) is an organ with a specialized immune system strictly regulated by the $\mathrm{BBB}$ that limits the entry of cells and molecules. In the steady state, the CNS is devoid of immune cells, thus immune responses in the CNS are coordinated primarily by resident astrocytes and microglia to maintain health of the CNS (Ransohoff \& Brown, 2012). The CNS-resident microglia express lower levels of the hematopoietic marker CD45 and antigen-presenting molecules like MHC class II (MHC-II) than their circulating monocytic counterparts (Ford, Goodsall, Hickey, \& Sedgwick, 1995; Hickman et al., 2013; Kreutzberg, 1996), and these differences help to distinguish these populations experimentally. Since the BBB limits entry of cells and molecules such as antibodies into the CNS, microglia must be able to recognize pathogens, act as antigen-presenting cells and recruit peripheral immune cells to the CNS, forming an important part of the connection between the central and peripheral immune system (Ransohoff \& Brown, 2012). This connection is vital for robust immune responses during CNS infection; however, microglia can also contribute to neurodegeneration during neurological disorders such as MS (Bogie et al., 2014), stroke (Patel, Ritzel, McCullough, \& Liu, 2013) and even psychiatric illnesses like schizophrenia (Laskaris et al., 2016).

When immune homeostasis in the CNS is disturbed, peripheral monocytes are recruited into the CNS to support the immune function of microglia; however, during hyper-active or autoimmune reactions recruited monocytes can aggravate the inflammatory response to a point where the response is no longer protective but instead detrimental to the host tissue (Jiang, Jiang, \& Zhang, 2014). In EAE and MS, infiltrating monocytes are abundant in demyelinating regions of the CNS and express high levels of MHC and co-stimulatory molecules indicative of an inflammatory state of activation known as classical activation (D. Y. S. Vogel et al., 2013; Yamasaki et al., 2014). In addition, activated microglia and macrophages secrete proinflammatory mediators that contribute to the formation of inflammatory lesions (Dendrou et al., 2015). In contrast to classical activation, microglia and monocytes can become alternatively activated and have regulatory functions important for repair and the resolution of inflammation (Xiaoming $\mathrm{Hu}$ et al., 2015). Alternatively activated microglia and macrophages are poor antigen-presenting cells, secrete anti-inflammatory cytokines and are essential for efficient remyelination of demyelinated lesions (Miron et al., 2013). 
The atypical antipsychotic clozapine currently used for treating schizophrenia has recently shown promise as an immune-modifying agent. Moreover, we have previously shown that risperidone and clozapine can reduce EAE disease and that risperidone suppressed the activation of microglia and macrophages in the CNS (D. O'Sullivan et al., 2014). Other studies show that atypical antipsychotic agents like risperidone and clozapine are able to suppress the classical activation of microglia (Bian et al., 2008; X. Hu et al., 2012; T. Kato et al., 2007). Given that clozapine readily crosses the BBB, it was hypothesized that clozapine is beneficial to EAE by inhibiting the activation of microglia and macrophages in the CNS. This chapter aims to investigate the effect of clozapine on microglia and macrophages during EAE to determine whether disease protection by clozapine treatment is associated with an altered state of activation.

\subsection{Aim}

This chapter studies how treatment with clozapine affects the activation of macrophages and microglia during EAE as a potential mechanism by which clozapine may reduce disease severity.

\subsubsection{Specific Aims}

1. To study how clozapine affects leukocyte infiltration into the CNS.

2. To study how clozapine treatment affects the activation of resident microglia and infiltrating monocytes 


\subsection{Results}

\subsubsection{Clozapine limits peripheral infiltration of leukocytes into the CNS 15 d.p.i}

It has been shown in the previous chapters that clozapine treatment effectively suppresses disease during EAE. Since EAE disease progression is for the most part driven by resident microglia and peripheral macrophages derived from circulating monocytes, infiltration of inflammatory cells into the spinal cord was measured by flow cytometry at peak disease (15 d.p.i). At this stage of disease, a remarkable discordance in disease score is evident between vehicle and clozapine-treated mice (Figure 5.1A). At this stage of disease, spinal cords were isolated and analysed by flow cytometry to distinguish the residing microglia (CD $45^{10} \mathrm{CD} 11 \mathrm{~b}^{10}$ ) from infiltrating macrophages $\left(\mathrm{CD} 45^{\mathrm{hi}} \mathrm{CD} 11 \mathrm{~b}\right.$; ; Figure 5.1B). This gating strategy effectively identifies the residing microglia from infiltrating macrophages as immunized EAE mice had significant proportions of macrophages whereas they were negligible in healthy controls (Figure 5.1C). Clozapine-treated mice had significantly lower monocytic infiltration into the spinal cord than vehicle controls (Figure 5.1C). Similarly, the proportion of lymphocytes $\left(\mathrm{CD} 45^{+} \mathrm{CD} 11 \mathrm{~b}^{-}\right)$in the spinal cord was also significantly lower in clozapine treated mice (Figure 5.1D). Finally, the severity of monocytic infiltration correlated to the clinical score of each individual mouse at the time of euthanasia (Figure 5.1E). Altogether, these results shows that clozapine effectively limits peripheral infiltration of inflammatory leukocytes into the CNS and is directly associated to the reduction in EAE disease severity. 
A

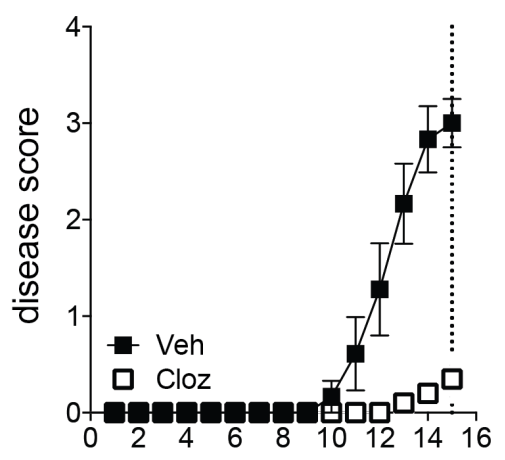

days post immunization

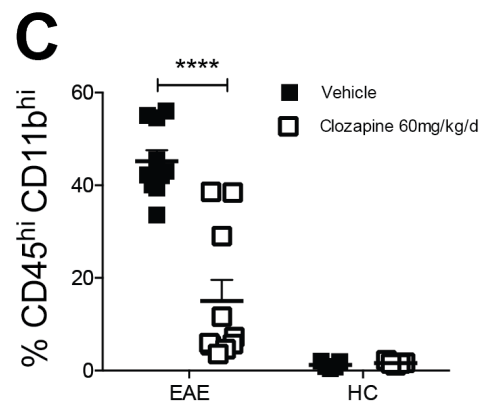

\section{B}
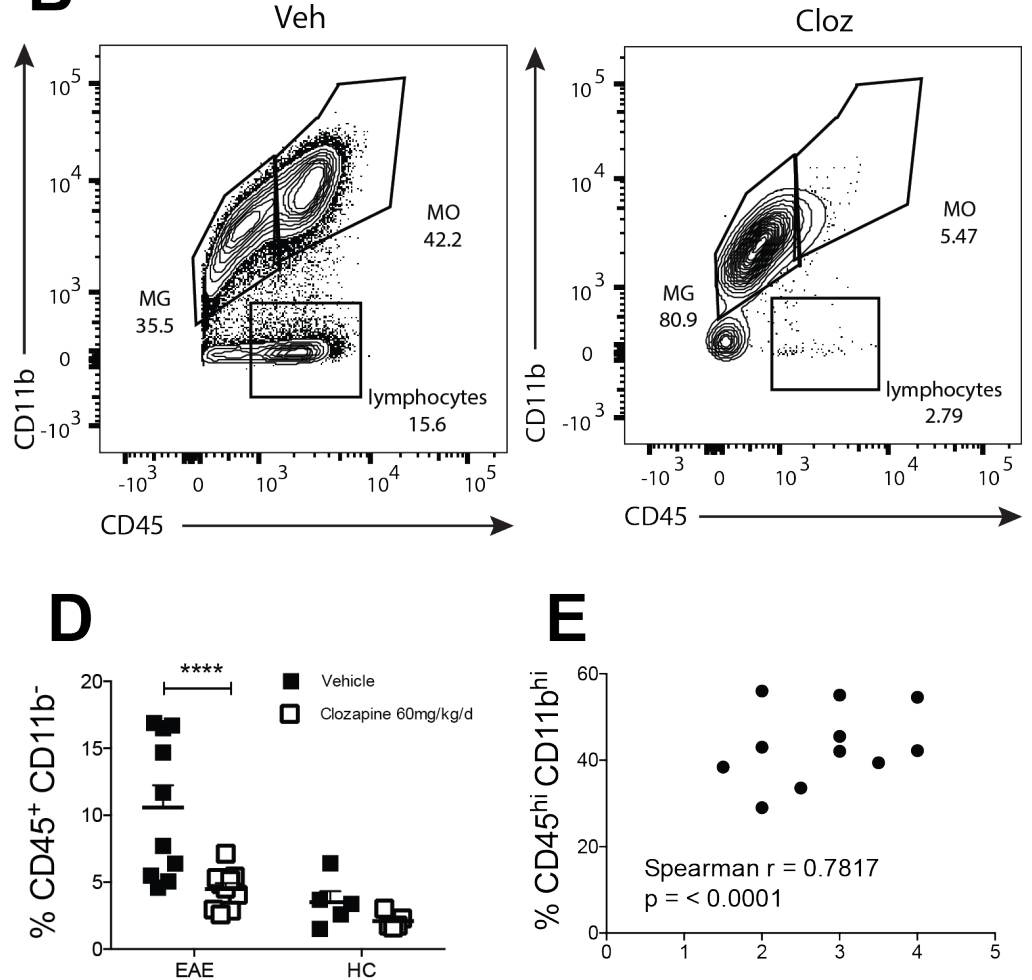

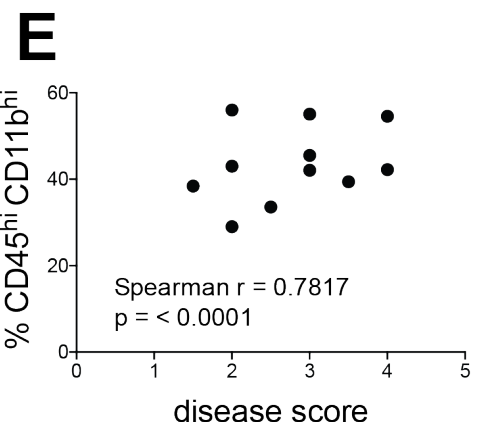

Figure 5.1 Clozapine limits CNS infiltration of leukocytes during EAE

A-E C57BL/6J mice were treated with clozapine $(60 \mathrm{mg} / \mathrm{kg} / \mathrm{day})$ or vehicle in their drinking water one day before EAE induction and A scored daily for disease (0; normal - 5; moribund). Shown are means and SEM of individual mice from two experiments $(n=10$ mice in each group). Mice were euthanized at 15 d.p.i indicated by a dotted line. B Representative dot plots of CD45 vs CD11b from spinal cord of vehicle (left) or clozapine treated mice (right) at 15 d.p.i (gated on single live cells; rationale for gating is shown in Appendix II). C Percentage of CD $45^{\text {hi }} \mathrm{CD} 11 \mathrm{~b}^{\mathrm{hi}}$ from CD $45^{+}$cells in the spinal cord at 15 d.p.i. Shown are means and SEM of individual mice $\left(n=5\right.$; HC and $n=10$; EAE). D Percentage of CD $45^{+}$CD $11 b^{-}$from CD $45^{+}$cells. Shown are means and SEM of individual mice $(n=5$ from one experiment; HC and combined $n=10$ from two experiments; EAE). E Percentage of CD $45^{\text {hi }} \mathrm{CD} 11 \mathrm{~b}^{\text {hi }}$ cells from CD $45^{+}$cells in the spinal cord at 15 d.p.i. vs. disease score correlation. Shown are means and SEM of individual mice from two experiments combined (combined $\mathrm{n}=10$ mice in each group). Statistical analyses were performed by two-way ANOVA and Sidak's multiple comparison test. $* * * * \mathrm{p}<0.0001$. 


\subsection{Clozapine treated mice express lower levels of inflammatory markers on microglia and CNS infiltrating monocytes 15 d.p.i}

Clozapine has previously been reported to inhibit the activation of microglia (X. Hu et al., 2012). Given that clozapine effectively limits peripheral infiltration of leukocytes into the CNS, the activation phenotype of the resident microglia and infiltrating macrophages were analysed by flow cytometry as a potential mechanism by which inflammatory recruitment to the spinal cord was limited. Using the gating strategy previously described (Figure 5.2A), the expression of antigen presentation and co-stimulatory molecules were measured on microglia and infiltrating monocytes to characterize their state of activation. At 15 d.p.i, resident microglia in clozapine-treated mice expressed lower levels of the lipopolysaccharide co-receptor CD14 than vehicle controls (Figure 5.2B). Expression of co-stimulatory molecules CD80 (Figure 5.2C) and CD86 (Figure 5.2D) and antigen-presenting molecule MHC class II (Figure 5.2E) were significantly lower in clozapine-treated mice. Altogether, these data show that mice treated with clozapine had microglia that were less inflammatory during peak disease.
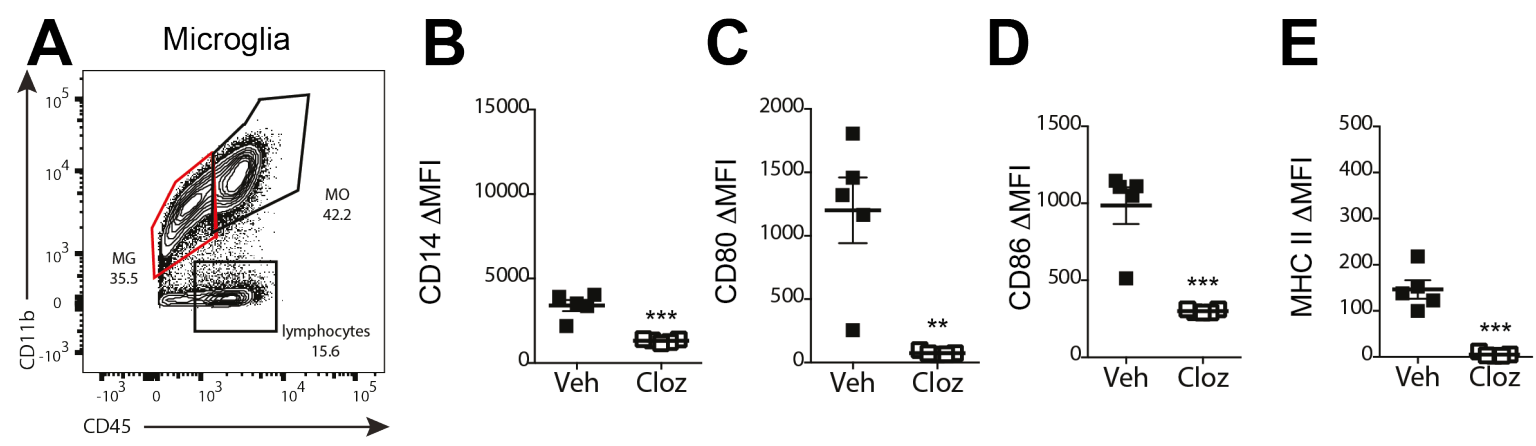

Figure 5.2 Clozapine treatment inhibits expression of activation markers on microglia 15 d.p.i

A-E C57BL/6J mice that were treated with clozapine $(60 \mathrm{mg} / \mathrm{kg} /$ day $)$ or vehicle in their drinking water one day before EAE induction were euthanized 15 d.p.i and spinal cord infiltration analysed by flow cytometry. A Gating strategy for resident microglia (red outline) in spinal cord 15 d.p.i (shown is a vehicle treated EAE mouse) B-E $\triangle$ MFI (MFI - Isotype control) of B CD14, C CD80, D CD86 and E IA/IE (MHC Class II), respectively by monocytes from vehicle or clozapine treated mice. Shown are means and SEM of individual mice ( $\mathrm{n}=5$ mice in each group) from one experiment. $* * * \mathrm{p}=<0.001$ by Student's t-test.

Infiltrating monocytes expressed much higher levels of CD14, CD80, CD86 and MHC Class II than CNS resident microglia as previously described. Like the microglia, infiltrating monocytes in clozapine-treated mice expressed significantly less CD14 (Figure 5.3B), CD80 
(Figure 5.3C), CD86 (Figure 5.3D) and MHC class II (Figure 5.3E) on the cell surface, showing that infiltrating monocytes are in a less inflammatory state in clozapine-treated mice.
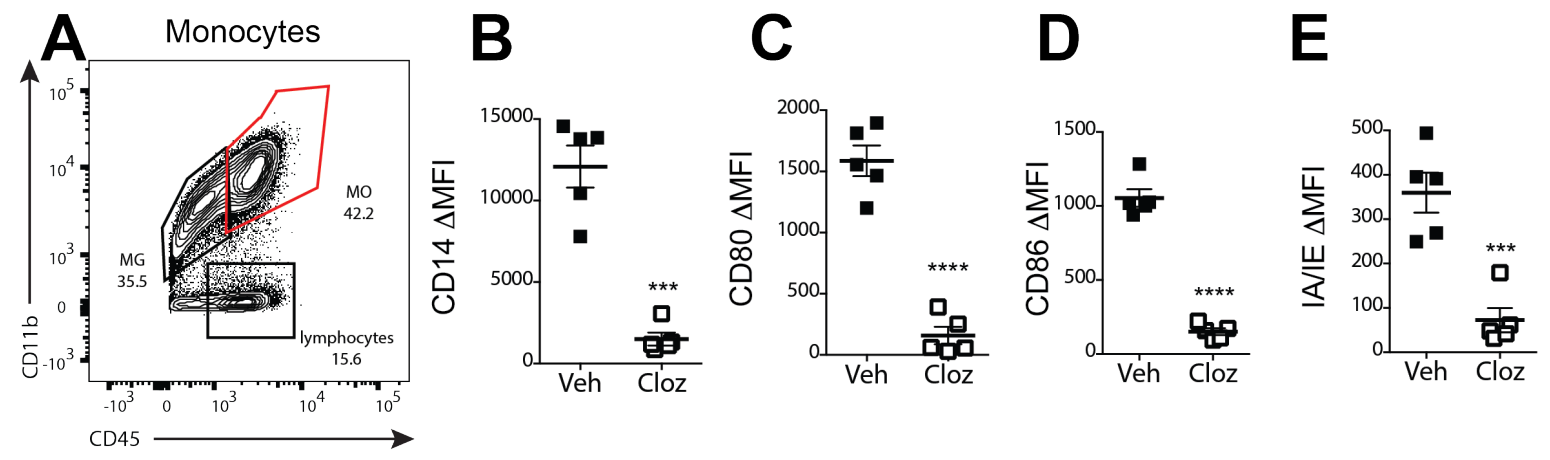

\title{
Figure 5.3 Clozapine inhibits expression of activation markers on infiltrating monocytes in the spinal cord 15 d.p.i.
}

\begin{abstract}
A-E C57BL/6J mice that were treated with clozapine $(60 \mathrm{mg} / \mathrm{kg} /$ day $)$ or vehicle in their drinking water one day before EAE induction were euthanized 15 d.p.i and spinal cord infiltration analysed by flow cytometry. A Gating strategy for identifying infiltrating monocytes (red outline) in the spinal cord 15 d.p.i (shown is a vehicle treated EAE mouse; rationale for gating strategy can be found in Appendix II) B-E $\Delta$ MFI (MFI - Isotype control) of B CD14, C CD80, D CD86 and E IA/IE (MHC Class II), respectively by monocytes from vehicle or clozapine treated mice. Shown are means and SEM of individual mice $(n=5$ mice in each group) from one experiment. $* * * \mathrm{p}=<0.001$ by Student's t-test.
\end{abstract}

\subsection{CNS infiltration of myeloid cells in clozapine treated mice parallels infiltration in vehicle treated mice}

To further investigate the effect of clozapine on the activation state of microglia and monocytes during EAE disease progression, spinal cord infiltrates were assessed at the start of the chronic phase of disease 20 d.p.i. (Figure 5.4A). Microglia and monocytes were identified in vehicle and clozapine-treated mice by flow cytometry using the gating strategy previously described (Figure 5.4B). Significant infiltration of monocytes was observed in EAE mice when compared to the healthy controls (Figure 5.4C). Interestingly, although clozapine-treated mice remained protected from disease 20 d.p.i, monocytic infiltration was not significantly different between clozapine and vehicle-treated mice (Figure 5.4C) although a slight decrease in lymphocytes was still evident (Figure 5.4D). 
A

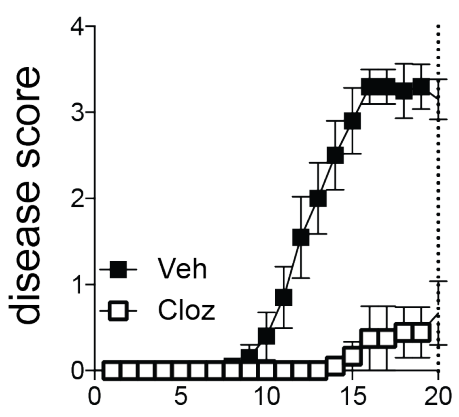

days post immunization
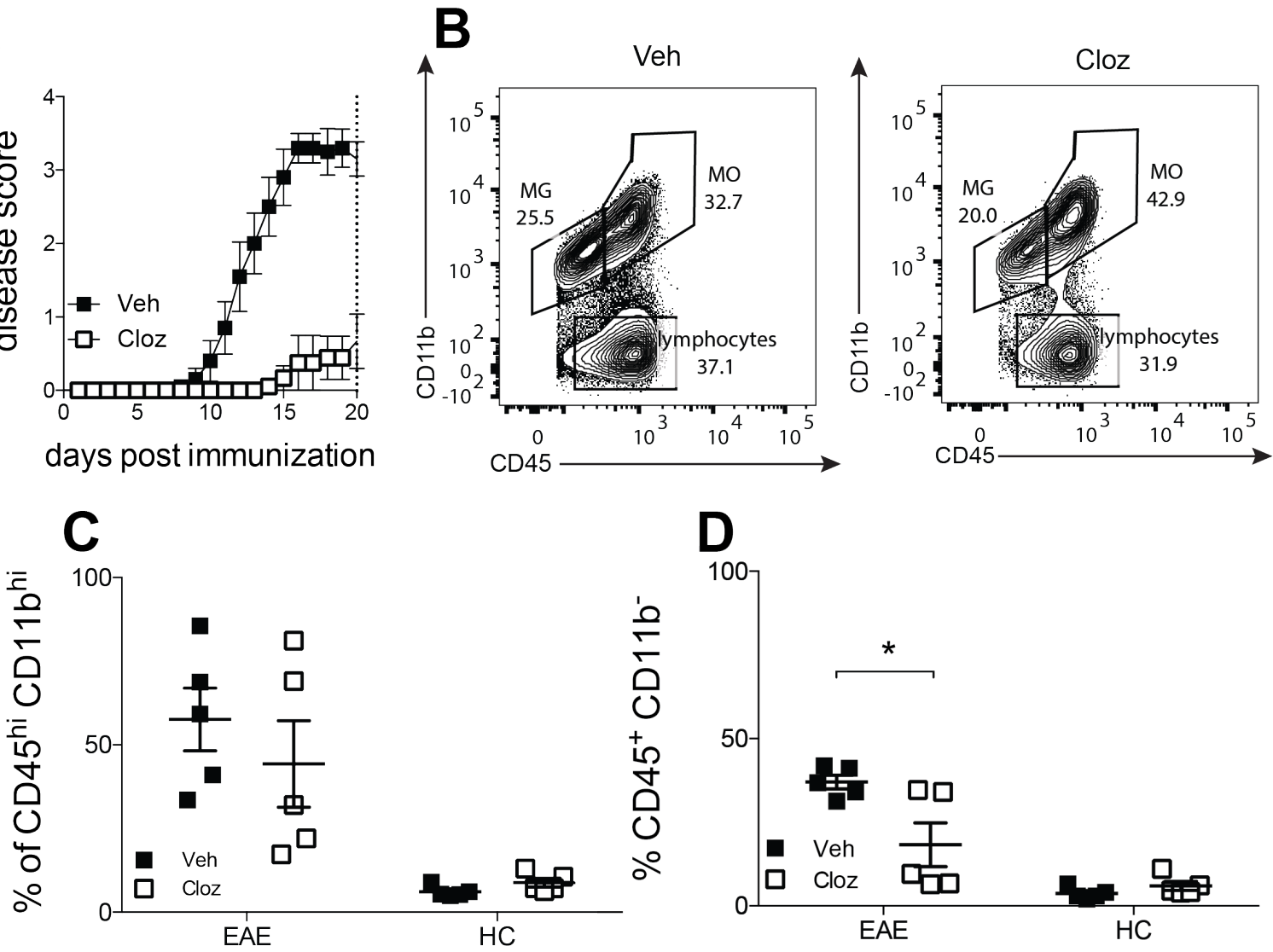

\section{Figure 5.4 No difference in CNS infiltration by 20 d.p.i}

A-D C57BL/6J mice were treated with clozapine $(60 \mathrm{mg} / \mathrm{kg} /$ day $)$ or vehicle in their drinking water one day before EAE induction and scored daily for disease (0; normal - 5; moribund). Shown are means and SEM of individual mice $(n=5$ mice in each group). B Representative dot plots of CD45 vs CD11b from spinal cord of vehicle (left) or clozapine-treated mice (right) at 20 d.p.i (gated on single live cells). C Percentage of CD45 ${ }^{\text {hi }}$ $\mathrm{CD} 11 \mathrm{~b}^{\mathrm{hi}}$ from $\mathrm{CD} 45^{+}$cells in the spinal cord at 20 d.p.i. Shown are means and SEM of individual mice $(\mathrm{n}=5$; $\mathrm{HC}$ and $\mathrm{n}=5$; EAE). D Percentage of CD $45^{+} \mathrm{CD} 11 \mathrm{~b}^{-}$from $\mathrm{CD} 45^{+}$cells. Shown are means and SEM of individual mice $(\mathrm{n}=5$; HC and $\mathrm{n}=10$; EAE). Statistical analyses by two-way ANOVA and Sidak's multiple comparison test. $* \mathrm{p}<0.05$.

\subsubsection{Clozapine inhibits the activation of microglia and infiltrating monocytes in the spinal cord 20 d.p.i.}

To understand how clozapine treated mice remained protected from disease despite having a similar proportion of infiltrating monocytes in the spinal cord, expression of activation markers on the resident microglia was measured (Figure 5.5A). EAE mice had significantly higher expression of MHC class II (Figure 5.5B) and CD86 (Figure 5.5D) than healthy controls whereas CD80 (Figure 5.5C) and CD40 (Figure 5.5E) were not significantly different. Interestingly, at this stage of disease, only microglial MHC class II expression was significantly 
lower in clozapine treated mice. These data show that clozapine inhibits the activation of microglia even at the chronic phase of disease, albeit to a lesser extent.
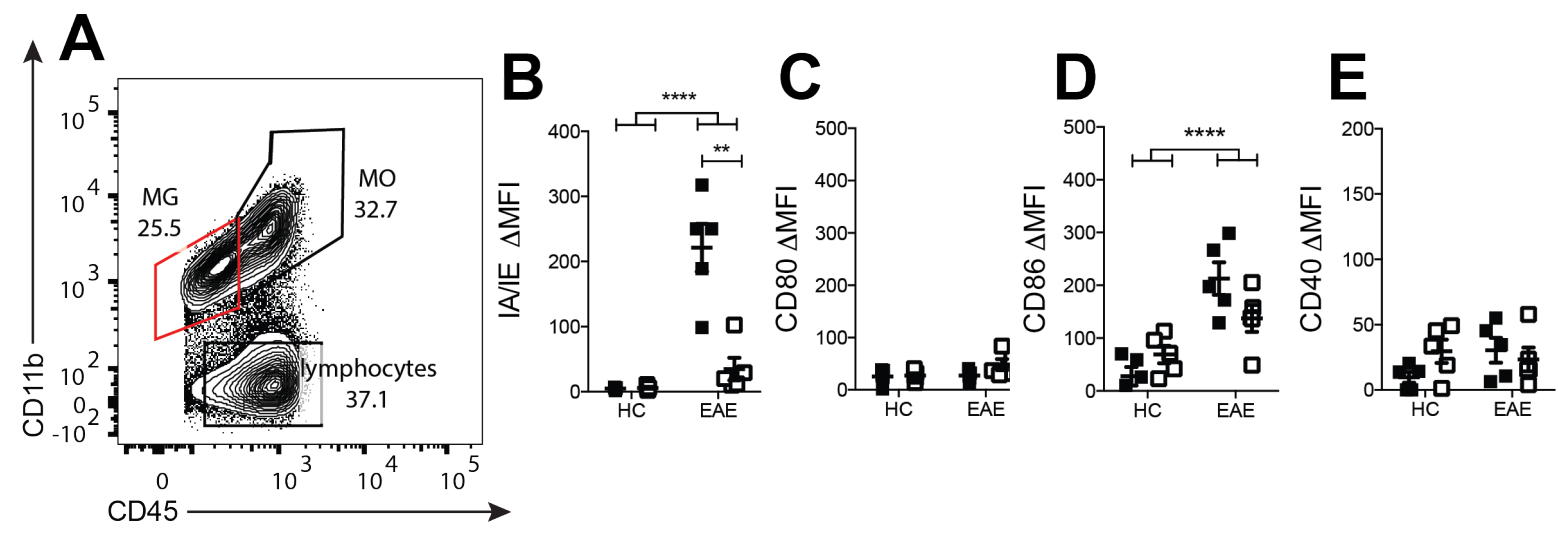

Figure 5.5 Clozapine inhibits the activation of microglia in the spinal cord 20 d.p.i

A-E C57BL/6J mice that were treated with clozapine $(60 \mathrm{mg} / \mathrm{kg} / \mathrm{day})$ or vehicle in their drinking water one day before EAE induction were euthanized 20 d.p.i and spinal cord infiltration analysed by flow cytometry. A Gating strategy for identifying resident microglia (red outline) in the spinal cord 20 d.p.i (shown is a vehicle treated EAE mouse; rationale for gating strategy can be found in Appendix II) B-E $\Delta$ MFI (MFI - Isotype control) of B IA/IE (MHC Class II), C CD80, D CD86 and E CD40 by monocytes from vehicle or clozapine treated mice. Shown are means and SEM of individual mice $(n=5$ mice in each group $)$ from one experiment. $* * * \mathrm{p}=<0.001$ by Student's t-test.

In the monocytic infiltrates (Figure 5.6A), EAE mice had significantly higher expression of MHC Class II (Figure 5.6B), CD80 (Figure 5.6C) and CD86 (Figure 5.6D) but not CD40 (Figure 5.6E) on infiltrating monocytes when compared to unimmunized mice. Similar to the effect on microglia, clozapine only suppressed the up-regulation of MHC Class II on infiltrating monocytes in the spinal cord (Figure 5.6B) while CD80, CD86 and CD40 were no longer affected by clozapine treatment. Altogether, although the effect of clozapine on altering activation is modest at 20 d.p.i, these results indicate that even though monocytic infiltration was similar in clozapine-treated mice, resident microglia and infiltrating monocytes were in a less inflammatory state during clozapine treatment and suggests that clozapine reduced the activation of microglia and monocytes during EAE. 


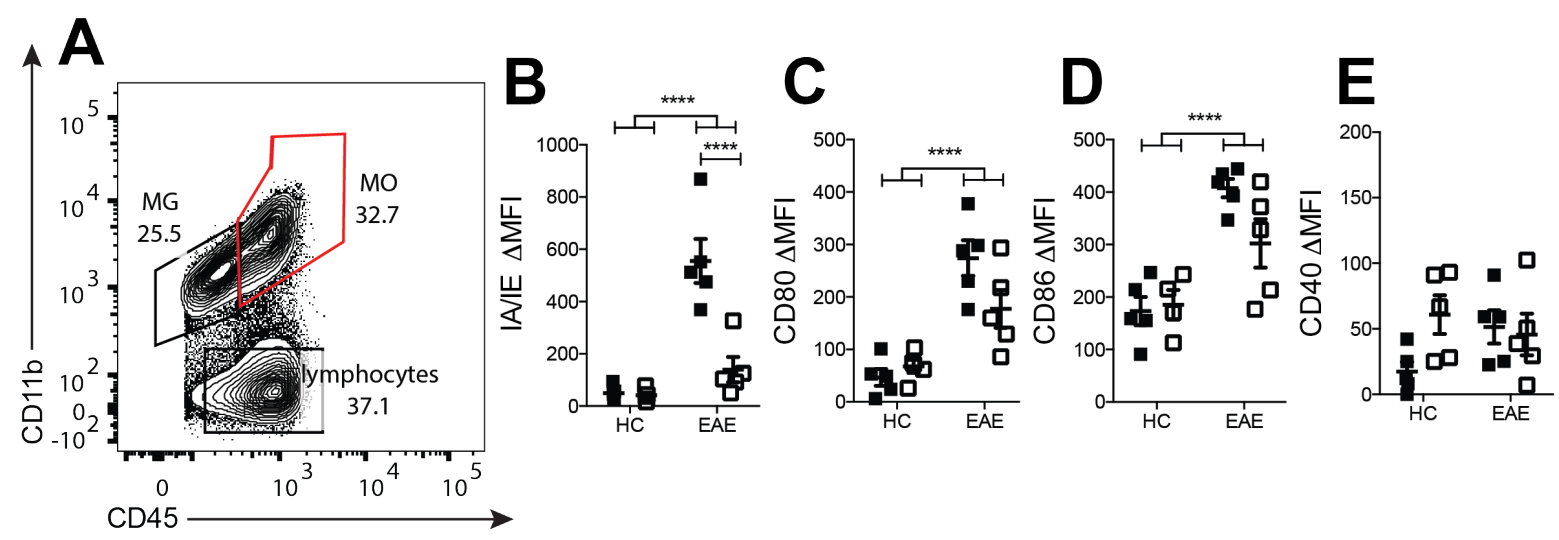

\section{Figure 5.6 Clozapine inhibits the activation of macrophages in the spinal cord 20 d.p.i}

A-E C57BL/6J mice that were treated with clozapine $(60 \mathrm{mg} / \mathrm{kg} /$ day $)$ or vehicle in their drinking water one day before EAE induction were euthanized 20 d.p.i and spinal cord infiltration analysed by flow cytometry. A Gating strategy for identifying resident infiltrating monocytes (red outline) in the spinal cord 20 d.p.i (shown is a vehicle treated EAE mouse; rationale for gating strategy can be found in Appendix II) B-E $\triangle$ MFI (MFI Isotype control) of B IA/IE (MHC Class II), C CD80, D CD86 and E CD40 by monocytes from vehicle or clozapine treated mice. Shown are means and SEM of individual mice $(n=5$ mice in each group) from one experiment. $* * * \mathrm{p}=<0.001$ by Student's t-test. 


\subsection{Discussion}

Recent studies indicate that CNS-resident microglia are crucial for the pathogenesis of inflammatory diseases of the CNS like multiple sclerosis, especially in the progressive forms (Mahad, Trapp, \& Lassmann). Indeed, previously published research as well as this study has shown that the resident microglia and peripherally-derived infiltrating macrophages upregulate inflammatory markers during EAE. This study aimed to investigate how clozapine reduced EAE disease and found that clozapine inhibited the inflammatory activation of both resident microglia and infiltrating monocytes.

Microglia in the resting-state generally express very low levels of MHC class II, CD80, CD86 and CD40, which is consistent with our current findings. During an inflammatory response however, microglia can up-regulate these molecules and gain function such as antigen presentation and co-stimulation analogous to peripheral macrophages. MHC class II expression is up-regulated in active MS lesions (D. Y. Vogel et al., 2013) and similarly during EAE (Eugene D. Ponomarev, Veremeyko, Barteneva, Krichevsky, \& Weiner, 2011). Interestingly, schizophrenia is strongly associated with certain MHC alleles (Schizophrenia Working Group of the Psychiatric Genomics, 2014). One of the most striking observations in this chapter is that clozapine significantly suppressed the expression of MHC class II on microglia as well as on the infiltrating monocytes, confirming previous reports of clozapine inhibiting the activation of microglia. Given that we have already shown risperidone and clozapine to directly affect the activation of macrophages in in vitro cultured macrophages, the reduced expression of MHC Class II and co-stimulatory molecules are possibly a direct effect of clozapine treatment. In addition, although this study has not completely addressed this possibility, there is still the potential that clozapine affects the magnitude of CD4 T cell "help" provided to macrophages and myeloid cells in the CNS by altering the expression of cytokines such as IFN- $\gamma$ and stimulatory molecules and therefore reducing the expression of inflammatory markers on CNS macrophages and microglia. Nevertheless, reduced expression of MHC class II in the CNS has great implications for both EAE and MS as myelin-reactive T cells from the periphery are thought to be re-activated upon entry into the $\mathrm{CNS}$, and is required for disease progression (Goverman, 2009; Platten \& Steinman, 2005). Moreover, MHC class II-expressing microglia are required for the induction of passive EAE (Hickey \& Kimura, 1988), highlighting its importance in EAE progression and suggesting that down-regulation by clozapine has the potential to limit disease severity. 
In addition to MHC class II interactions, T cells require co-stimulation with CD80 and CD86 molecules for activation through CD28. This interaction is clearly important for EAE progression and is demonstrated when adoptively transferred encephalitogenic $\mathrm{T}$ cells were shown to be more prone to apoptotic cell death when in the CNS of CD80 and CD86 deficient mice (Chang et al., 2003). Our results show that clozapine is able to suppress the expression of CD80 and CD86 by microglia and macrophages early during EAE, and this suppression may contribute to the protective effect of clozapine on EAE disease.

In addition to an overall reduction in disease severity, clozapine effectively delayed the entry of infiltrating monocytes into the CNS. Under homeostatic conditions, the CNS is generally devoid of circulating leukocytes due to the BBB. Therefore, immune surveillance in the CNS is conducted by MHC class II-expressing cells, predominantly microglia (Bo et al., 1994) which secrete chemokines such as CCL2 and CCL3 to recruit peripheral cells to the CNS (Juedes, Hjelmström, Bergman, Neild, \& Ruddle, 2000; Yamasaki et al., 2014). During EAE, the resident microglia in the CNS are activated, and this activation precedes any infiltration of peripheral cells. One study suggests that the early activation of microglia occurs by activation from GM-CSF produced by myelin-specific T cells in the periphery (E. D. Ponomarev et al., 2007). Bone marrow chimeric mice that are deficient for CCL2 in microglia are resistant to EAE (Dogan et al., 2008), which indicates that microglial activation and production of chemokines is absolutely essential for recruitment of inflammatory cells to induce EAE. Interestingly, because it was shown in the previous chapter that clozapine did not affect the development of robust antigen-specific $\mathrm{T}$ cell responses in the periphery, clozapine may limit the infiltration of peripheral leukocytes into the CNS by inhibiting the activation of microglia during the induction phase of EAE.

It is not clear why microglia are chronically active in progressive MS, however, this occurrence is present in many other neurological and psychiatric disease including schizophrenia. Like progressive forms of MS, schizophrenia is characterized with the loss of grey and white matter and consequential loss of connectivity that is associated with increased microglial activation. These studies have recently been extensively reviewed (Laskaris et al., 2016) and some investigators propose that microglia are central to schizophrenia related neurodegeneration (Rački et al.). Interestingly, treatments such as minocycline targeting the activation of microglia (Kobayashi et al., 2013) have shown promise in treating schizophrenia symptoms 
(Liu et al., 2014), even showing some additive effect when given in conjunction to atypical antipsychotic agents (Miyaoka et al., 2008). Likewise, atypical antipsychotic agents suppress microglial activation directly (X. Hu et al., 2012; D. O'Sullivan et al., 2014) and some investigators believe that their anti-inflammatory effect plays a role in reducing the psychiatric symptoms of schizophrenia and neurodegeneration that arise from chronic microglial activation (Monji et al., 2009).

\subsection{Conclusions}

Overall, the results in this chapter show that clozapine was able to inhibit the inflammatory activation of microglia and monocytes in the CNS and was associated with a reduced severity of disease. These data indicate that clozapine may be an effective and novel approach for the treatment of progressive MS, where inflammation and damage in the CNS is perpetuated by chronic microglial activation and little peripheral involvement and this approach warrants future investigation into this clinical promise for MS patients. Finally, this work has broader relevance to psychiatry as the mechanism of action of clozapine is still debated. Here, we showed that clozapine has potent immune-modulating properties that can target the microglia, which are known to be dysregulated during psychiatric illness like schizophrenia, and this study supports the hypothesis that atypical antipsychotic agents are beneficial in schizophrenia due to their ability to modify microglial activation. 


\section{Chapter 6: The effect of clozapine on macrophage activation}




\subsection{Introduction}

Atypical antipsychotic agents are increasingly being recognized for their immune modulating properties. In particular, risperidone has previously been shown to inhibit the production of NO by IFN- $\gamma$-activated microglia (Takahiro Kato, Akira Monji, Sadayuki Hashioka, \& Shigenobu Kanba, 2007). In addition, atypical antipsychotic agents such as risperidone and clozapine suppressed pro-inflammatory TNF- $\alpha$ while increasing anti-inflammatory IL-10 in the serum of LPS-challenged mice whereas the typical antipsychotic haloperidol only had a modest effect (Sugino et al., 2009). It has previously been shown that clozapine protects neurons from inflammatory damage induced by lipopolysaccharide (LPS) by reducing the production of reactive oxygen species, nitric oxide and TNF- $\alpha$ by microglia (X. Hu et al., 2012). Immune-modulating effects have also been observed for other atypical antipsychotic agents like quetiapine, which has been shown to reduce EAE disease (Mei et al., 2012). We have shown that risperidone and clozapine are effective at reducing disease in EAE whereas sulpiride is not (D. O'Sullivan et al., 2014). Although sulpiride is an atypical antipsychotic, it is a selective antagonist for D2 like receptors (Caley \& Weber, 1995) similar to the typical antipsychotic haloperidol, suggesting that the immune-modifying effect of atypical antipsychotic agents may be a consequence of targeting a wider range of neuroreceptors other than D2.

All of the neuroreceptors targeted by antipsychotic agents are g-protein coupled receptors (GPCR) which typically signal through phospholipase C (PLC), protein kinase B (Akt) and cyclic-AMP (cAMP) pathways. All of these pathways can be involved in immune responses and thus any change in the pathways may mediate immunomodulatory effects. Since atypical antipsychotic agents antagonize a range of receptors with different binding affinities, it makes their precise mechanism of action difficult to resolve experimentally. In any case, atypical antipsychotic agents like clozapine have been shown to regulate cyclic-AMP-dependent protein kinase A (PKA) activity (Turalba, Leite-Morris, \& Kaplan, 2004) and up-regulate Akt phosphorylation in the brain of mice (F. Panariello et al., 2012; Roh et al., 2007; Schmid, Streicher, Meltzer, \& Bohn, 2014), inhibiting glycogen synthase kinase-3 (GSK3) activity downstream ( $\mathrm{Li}$ et al., 2007). Since atypical antipsychotic agents such as clozapine have been found to act as agonists at 5-HT2a receptors (Schmid et al., 2014), they also have the potential to alter PLC signalling through the $\mathrm{G} \alpha_{\mathrm{q}}$ subunit. Although many signalling pathways are regulated by GPCR's, GSK-3 $\beta$ has been the most notably described as a key target of 
psychiatric drugs like atypical antipsychotic agents and anti-depressants (J. M. Beaulieu, Gainetdinov, \& Caron, 2009), many of which have immune modifying properties (Al-Amin, Nasir Uddin, \& Mahmud Reza, 2013; Raghavendra, Lee, \& Parameswaran, 2014; Sacre, Medghalchi, Gregory, Brennan, \& Williams, 2010). In addition, GSK-3 $\beta$ is central in regulating the cytokine production profile of macrophages during toll-like receptor signalling (Ko \& Lee, 2016), indicating that this enzyme plays a significant role in the activation of macrophages. Interestingly, psychiatric drugs that are understood to modulate GSK-3 $\beta$ such as lithium, atypical antipsychotic agents and fluoxetine have all been shown to reduce EAE disease (De Sarno et al., 2008; Mei et al., 2012; D. O'Sullivan et al., 2014; Yuan et al., 2012), although the mechanism is not yet understood. We have shown that clozapine inhibits the activation of macrophages and microglia in the CNS during EAE and is a potential mechanism by which clozapine reduces clinical disease. However, the mechanism by which clozapine may inhibit the activation of macrophages and microglia in the CNS is unknown, despite its discovery almost 60 years ago (Grover, Dutt, \& Avasthi, 2010).

Much like peripheral, tissue-resident macrophages, CNS-resident macrophages or microglia are known to maintain tissue homeostasis by surveying the brain parenchyma for insults or damage (Nimmerjahn, Kirchhoff, \& Helmchen, 2005), acting as the first line of defence during infection. Although microglia have tissue-specific functions such as synaptic pruning to maintain neuronal networks (Tremblay, 2011), they are also very similar to peripheral macrophages by having phagocytic capacity, ability to present antigens on MHC Class II, express co-stimulatory molecules for activation of $\mathrm{T}$ cells (Kettenmann, Hanisch, Noda, \& Verkhratsky, 2011) and even express toll-like receptors (Gurley et al., 2008). Moreover, peripheral injection with LPS is sufficient to activate microglia in the CNS (Z. Chen et al., 2012) highlighting their role as responders to insults. In this chapter, the effects of clozapine on the activation of macrophages and microglia are investigated by stimulating primary macrophages with lipopolysaccharide, as an in vitro model of inflammation to gain understanding into the effects of clozapine on the cell signalling network. 


\subsection{Aims}

This chapter aims to elucidate the mechanism by which clozapine can suppress the activation of macrophages and microglia.

\subsubsection{Specific aims}

1. Determine the effect of clozapine on the activation of LPS-stimulated macrophages as an in vitro model of inflammation

2. Determine the signaling mechanism by which clozapine alters LPS-induced cytokine profile in macrophages 


\subsection{Results}

\subsubsection{Macrophages expressed D1R, D2R and $\beta$-adrenergic receptors}

To show that clozapine has the potential to alter the activation of macrophages directly, the expression of the dopaminergic receptors D1 and D2 were assessed on mouse macrophages. Splenic white pulp macrophages were identified as $\mathrm{CD}_{4} 5^{+} \mathrm{CD} 11 \mathrm{~b}^{+} \mathrm{F} 4 / 80^{-}$. Macrophages from healthy mice were found to express D1R (Figure 6.1A) and D2R (Figure 6.1B). EAE had no effect on the expression of D1R and D2R on splenic macrophages at 40 d.p.i. These results show that mouse macrophages express dopamine receptors consistent with previously published studies. 

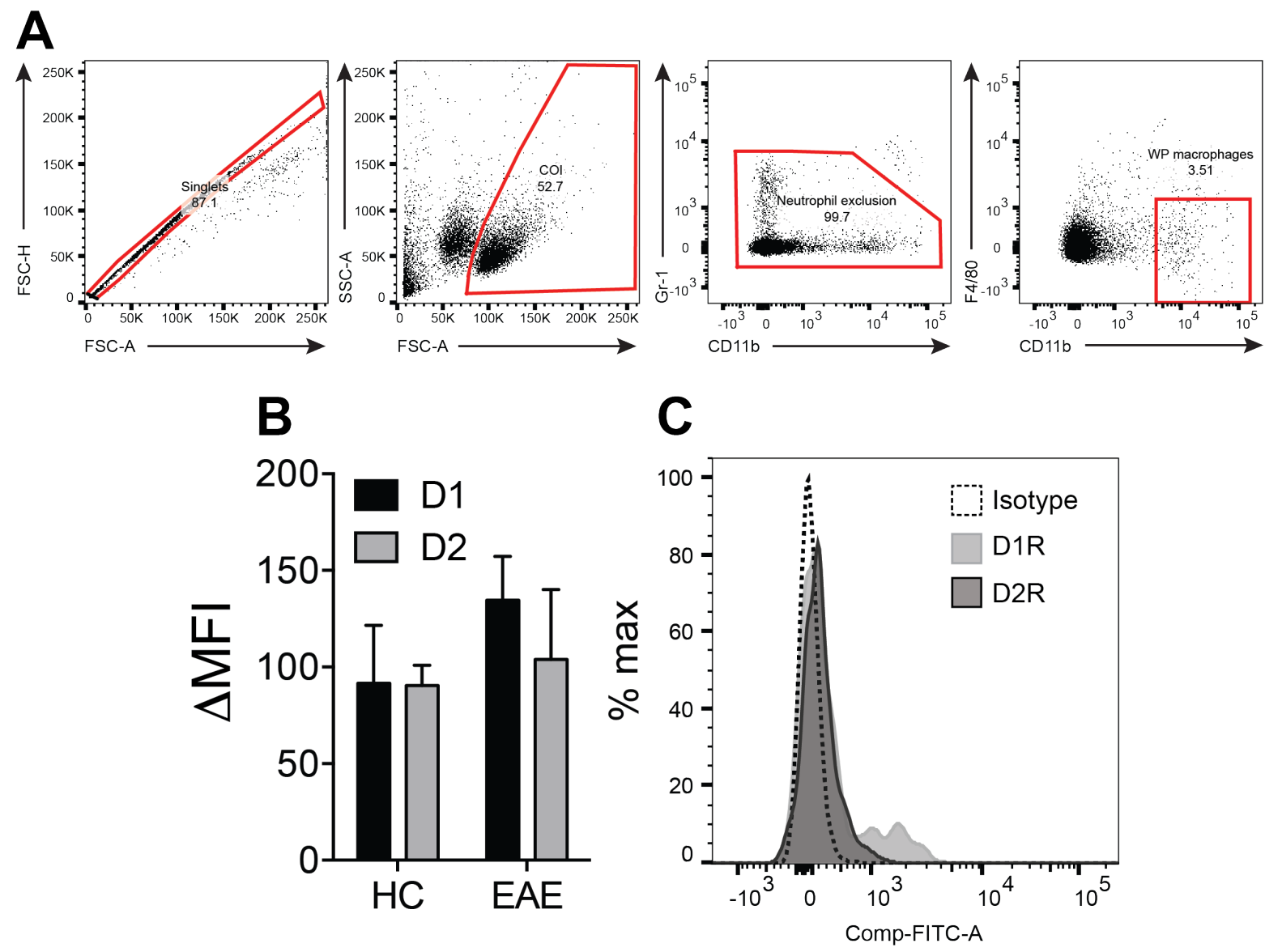

\section{Figure 6.1 White pulp macrophages express D1R and D2R}

A-C C57BL6/J mice were immunized for EAE and euthanized 40 d.p.i for analysis of dopamine receptors on white pulp macrophages. A Gating strategy to identify white pulp macrophages (gating shown in red from left to right). First, single cells were gated using FSC-A v FSC-H followed by gating for cells of interest by size and granularity using FSC-A vs SSC-A. Neutrophils were excluded by high expression of CD11b and Gr-1. White pulp macrophages were then gated as $\mathrm{CD} 11 \mathrm{~b}^{+} \mathrm{F} 4 / 80^{-}$cells and analysed for dopamine receptor expression (B). Shown are means and SEM of individual mice from one experiment ( $\mathrm{n}=4$ mice in each group). Statistical analyses by two-way ANOVA. Representative histogram of D1R and D2R expression on white pulp macrophages compared to isotype control from a healthy control mouse.

\subsection{Dopamine inhibited IL-12 and increased IL-10 secretion in LPS stimulated macrophages}

We have shown that murine macrophages express dopaminergic receptors consistent with previously published reports. To investigate the effect of dopamine on LPS-stimulated macrophages, dopamine was added to the culture medium at increasing concentrations. Consistent with previously published studies from our laboratory, IL-12 was decreased by dopamine from $20 \mu \mathrm{M}$ in a concentration dependent manner when stimulated by LPS whilst 
no IL-12 was detectable when macrophages were unstimulated regardless of the dopamine concentration (Figure 6.2A). IL-10 was increased by dopamine from $20 \mu \mathrm{M}$, and this increase was maintained at higher concentrations. Similar to IL-12, no IL-10 is detectable when macrophages were unstimulated regardless of the dopamine concentration (Figure 6.2B). Cell viability measured by MTT metabolism indicated that dopamine did not cause reduced cell viability in LPS-stimulated macrophages even at the high concentration of $100 \mu \mathrm{M}$ whereas 20 $\mu \mathrm{M}$ dopamine significantly reduced MTT metabolism in unstimulated macrophages (Figure 6.2C).
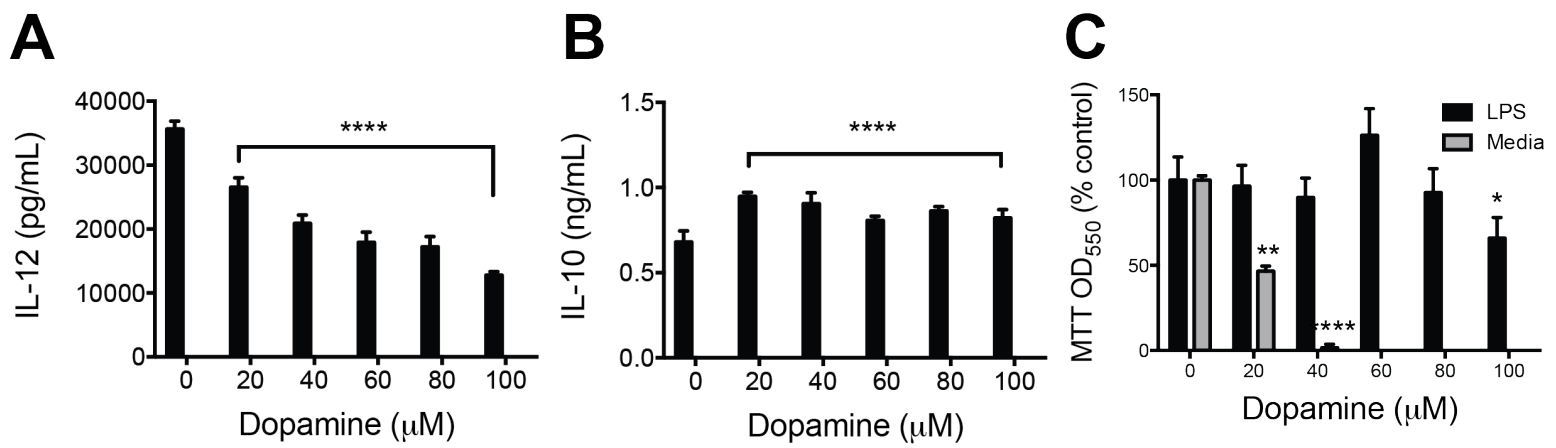

Figure 6.2 Dopamine inhibits IL-12 and increases IL-10 secretion without affecting viability

A-C BMMØ were plated at 100,000 cells/well, were primed with IFN- $\gamma(20 \mathrm{U} / \mathrm{mL})$ and stimulated with lipopolysaccharide for 24 hours in increasing concentrations of dopamine. IL-12 (A) and IL-10 (B) in the supernatants were measured by ELISA. Cell viability was measured by MTT assay presented as percent of vehicle control $(\mathbf{C})$. Shown are means and SEM of triplicate wells from one experiment representative of three. Statistical analyses performed using a two-way ANOVA and Sidak's multiple comparisons test. * p $<0.05, * *$ $\mathrm{p}<0.01, * * * \mathrm{p}<0.001$ and $* * * * \mathrm{p}<0.0001$

\subsection{Clozapine suppressed IL-12 and increased IL-10 production in lipopolysaccharide stimulated primary macrophages}

To classically activate macrophages in vitro, bone-marrow-derived macrophages were primed overnight with IFN- $\gamma$ and then stimulated with LPS (Mosser \& Zhang, 2008) in the presence of increasing concentrations of clozapine for 24 hours to examine the effect of clozapine on LPS-induced cytokine production as we have previously published (L. Green et al., 2017; D. O'Sullivan et al., 2014). After 24 hours of LPS stimulation, IL-12 and IL-10 are readily detectable in the supernatant (Figure 6.3A-B). IL-12 is significantly decreased from $10 \mu \mathrm{M}$ 
clozapine in a concentration dependent manner (Figure 6.3A). In contrast, IL-10 in the supernatant significantly increases from $20 \mu \mathrm{M}$ clozapine in a concentration dependent manner (Figure 6.3B). To verify that altered cytokine levels in the supernatant were not due to a loss in cell viability, an MTT metabolism assay was performed after each experiment and these experiments show that no decrease in cell viability is observed below $80 \mu \mathrm{M}$ clozapine (Figure 6.3C), demonstrating that the decrease in IL-12 and increase in IL-10 was not due to altered cell viability. Similarly, clozapine is effective at altering LPS-induced cytokine production in the RAW264.7 cell line commonly used for studying macrophages in vitro. Decreased IL-12 production was detectable from $5 \mu \mathrm{M}$ onwards (Figure 6.4A) without impairing MTT metabolism (Figure 6.4B). These data show that clozapine directly altered the activation profile of macrophages towards a less inflammatory phenotype. 

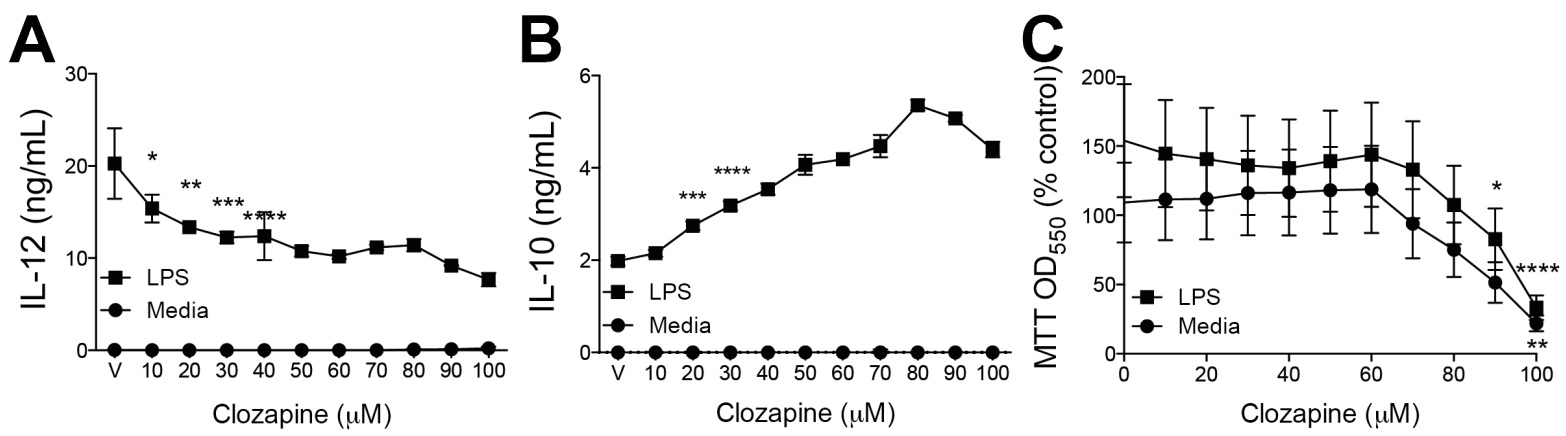

Figure 6.3 Clozapine inhibits secretion of IL-12 and increases IL-10 in lipopolysaccharide stimulated bone marrow derived macrophages

A-C BMMØ were plated at 100,000 cells/well, were primed with IFN- $\gamma(20 \mathrm{U} / \mathrm{mL})$ and stimulated with lipopolysaccharide for 24 hours in increasing concentrations of clozapine. IL-12 (A) and IL-10 (B) in the supernatants were measured by ELISA. Cell viability was measured by MTT assay presented as percent of vehicle control (C). Shown are means and SEM of triplicate wells from one experiment representative of three. Statistical analyses performed using a two-way ANOVA and Dunnett's multiple comparisons test. ${ }^{*} \mathrm{p}<0.05$, $* * \mathrm{p}<0.01, * * * \mathrm{p}<0.001$ and $* * * * \mathrm{p}<0.0001$
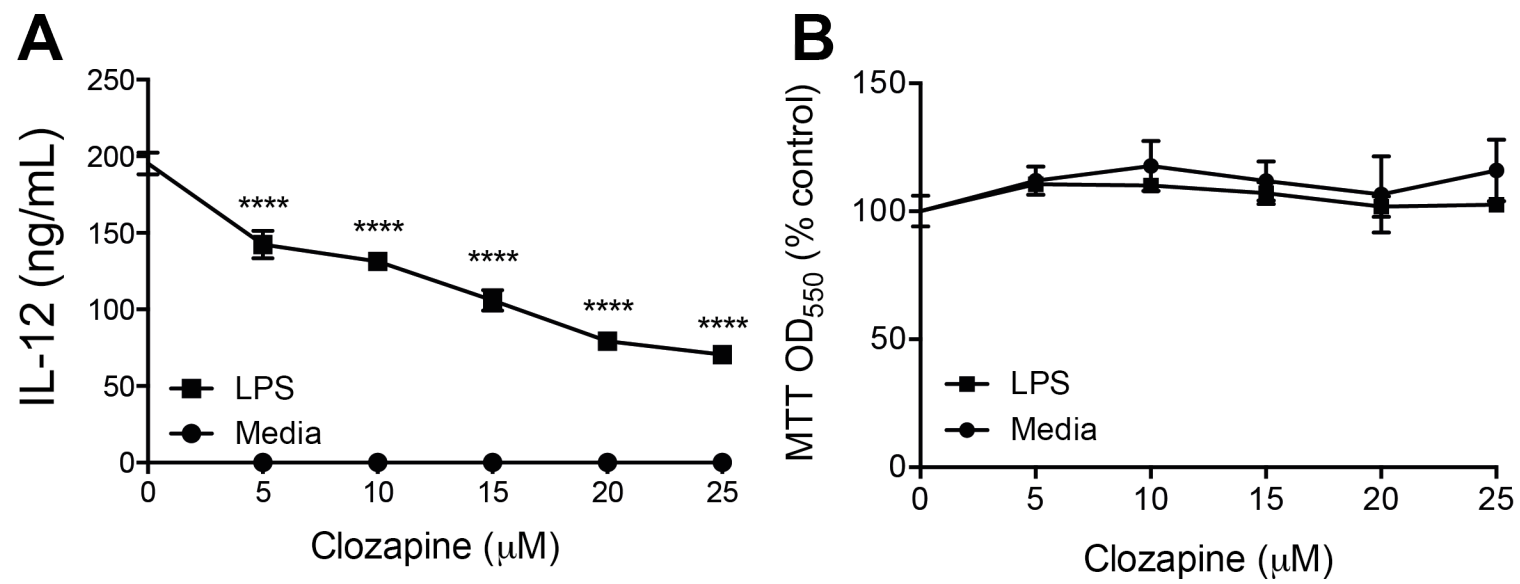

Figure 6.4 Clozapine inhibits secretion of IL-12 in lipopolysaccharide stimulated RAW264.7 cells

A-B RAW264.7 cells were plated at 50,000 cells/well, primed with IFN- $\gamma(20 \mathrm{U} / \mathrm{mL})$ and stimulated with lipopolysaccharide for 24 hours in increasing concentrations of clozapine. IL-12 (A) in the supernatants were measured by ELISA. Cell viability was measured by MTT assay presented as percent of vehicle control (B). Shown are means and SEM of triplicate wells from one experiment representative of three. Statistical analyses performed using a two-way ANOVA and Dunnett's multiple comparisons test. * $\mathrm{p}<0.05, * * \mathrm{p}<$ $0.01, * * * \mathrm{p}<0.001$ and $* * * * \mathrm{p}<0.0001$ 


\subsubsection{Risperidone suppressed IL-12 and increased IL-10 production in macrophages}

To investigate whether another atypical antipsychotic had a similar effect to clozapine, risperidone was added to the culture medium of bone marrow-derived macrophages stimulated with LPS at increasing concentrations to determine the effect of risperidone on macrophages directly. As previously published (D. O'Sullivan et al., 2014), IL-12 production after 24 hours of LPS stimulation was attenuated from $20 \mu \mathrm{M}$ risperidone (Figure 6.5A) whereas IL-10 production was significantly increased (Figure 6.5B). IL-10 production increased in a concentration-dependent manner. No alteration in MTT metabolism was observed up to $60 \mu \mathrm{M}$ (Figure 6.5C) consistent with our previously published results. These results confirm our previous finding that risperidone alters the activation of macrophages towards a less inflammatory phenotype.

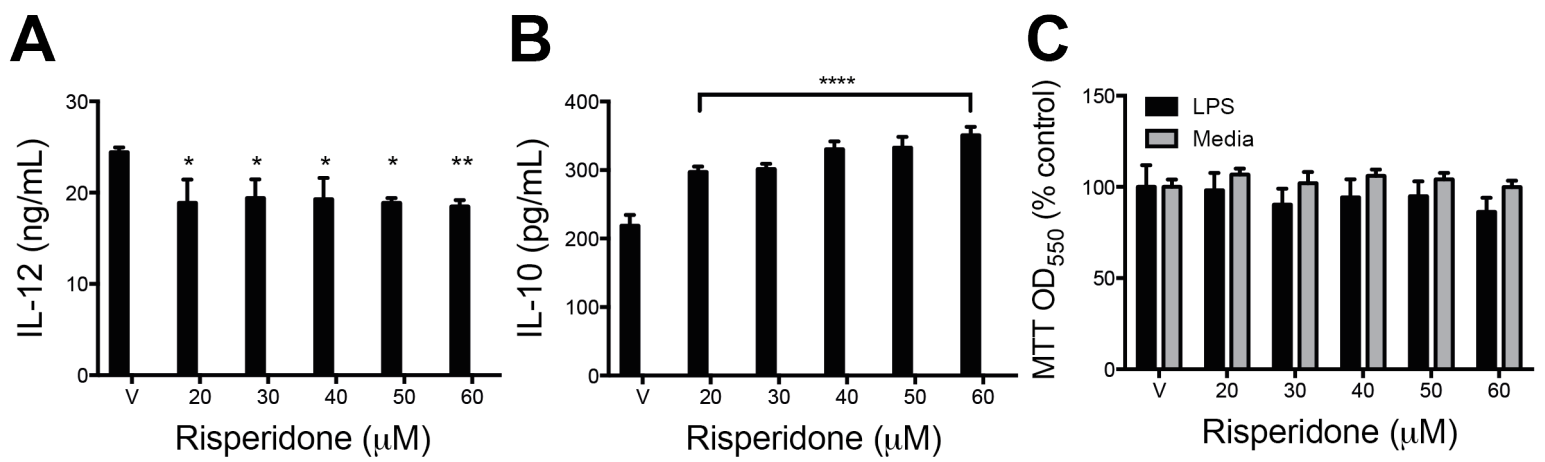

Figure 6.5 Risperidone suppresses IL-12 and increases IL-10 secretion in LPS stimulated macrophages

A-C BMMØ were plated at 100,000 cells/well, were primed with IFN- $\gamma(20 \mathrm{U} / \mathrm{mL})$ and stimulated with lipopolysaccharide for 24 hours in increasing concentrations of risperidone. A IL-12 and B IL-10 in the supernatants were measured by ELISA. C Cell viability was measured by MTT assay presented as percent of vehicle control. Shown are means and SEM of triplicate wells from one experiment representative of three. Statistical analyses performed using a two-way ANOVA and Dunnett's multiple comparisons test. ${ }^{*} \mathrm{p}<0.05$, $* * \mathrm{p}<0.01, * * * \mathrm{p}<0.001$ and $* * * * \mathrm{p}<0.0001$. 


\subsubsection{Forskolin inhibited secretion of IL-12 in macrophages}

D1R and D2R couple to G $\alpha$ protein subunits, which activate (D1R) or inhibit (D2R) cAMP signalling. To investigate whether altered cytokine production by clozapine was mediated by altered cAMP signalling, it was first examined whether increasing cAMP directly has an effect on IL-12 production in this model. Bone-marrow derived macrophages were primed overnight with IFN- $\gamma$ and stimulated with LPS in increasing concentration of forskolin and as expected, forskolin reduced the secretion of IL-12 in the supernatant significantly from $6.25 \mu \mathrm{M}$ onwards after 24 hours (Figure 6.6A). MTT metabolism was only significantly impaired at $100 \mu \mathrm{M}$ forskolin after 24 hours (Figure 6.6B) demonstrating that the loss in IL-12 production was not due to impaired cell viability. These results are consistent with previous reports that demonstrate that cAMP elevation alters the cytokine production profile of macrophages (Feng et al., 2002).
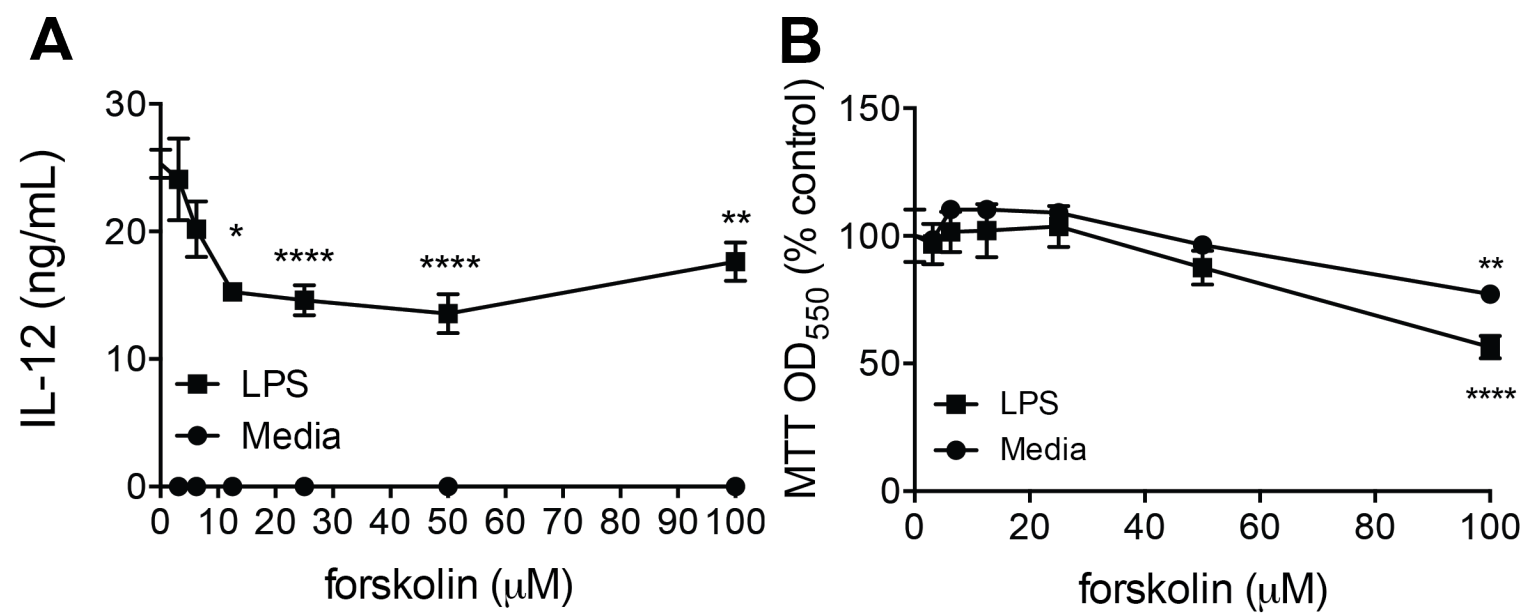

Figure 6.6 Forskolin inhibits secretion of IL-12 in LPS stimulated macrophages

A-B BMMØ were plated at 100,000 cells/well, were primed with IFN- $\gamma(20 \mathrm{U} / \mathrm{mL})$ and stimulated with lipopolysaccharide for 24 hours in increasing concentrations of forskolin. IL-12 (A) in the supernatants were measured by ELISA. Cell viability was measured by MTT assay presented as percent of vehicle control (B). Shown are means and SEM of triplicate wells from one experiment. Statistical analyses performed using a twoway ANOVA and Dunnett's multiple comparisons test. * $\mathrm{p}<0.05, * * \mathrm{p}<0.01, * * * \mathrm{p}<0.001$ and **** $\mathrm{p}<$ 0.0001 . 


\subsubsection{Clozapine did not alter cAMP production in lipopolysaccharide-stimulated primary macrophages}

Given that clozapine has potential to alter cAMP signalling in macrophages and that cAMP signalling can suppress IL-12 production, it was questioned whether clozapine suppressed IL12 production in LPS-stimulated macrophages in a cAMP-dependent manner. To address this, intracellular cAMP accumulation after LPS stimulation in the presence of $20 \mu \mathrm{M}$ clozapine was time-resolved up to 60 minutes post-LPS. Dopamine, which we have shown previously to reduce IL-12 production (D. O'Sullivan et al., 2014), increased production of cAMP in macrophages was detected at 15 and 45 minutes of LPS stimulation (Figure 6.7). When clozapine was added to the culture, no measurable effect was observed on cAMP up to 60 minutes post-stimulation (Figure 6.7) indicating that although clozapine can inhibit the production of IL-12, it did so in a manner that was independent of cAMP signalling.

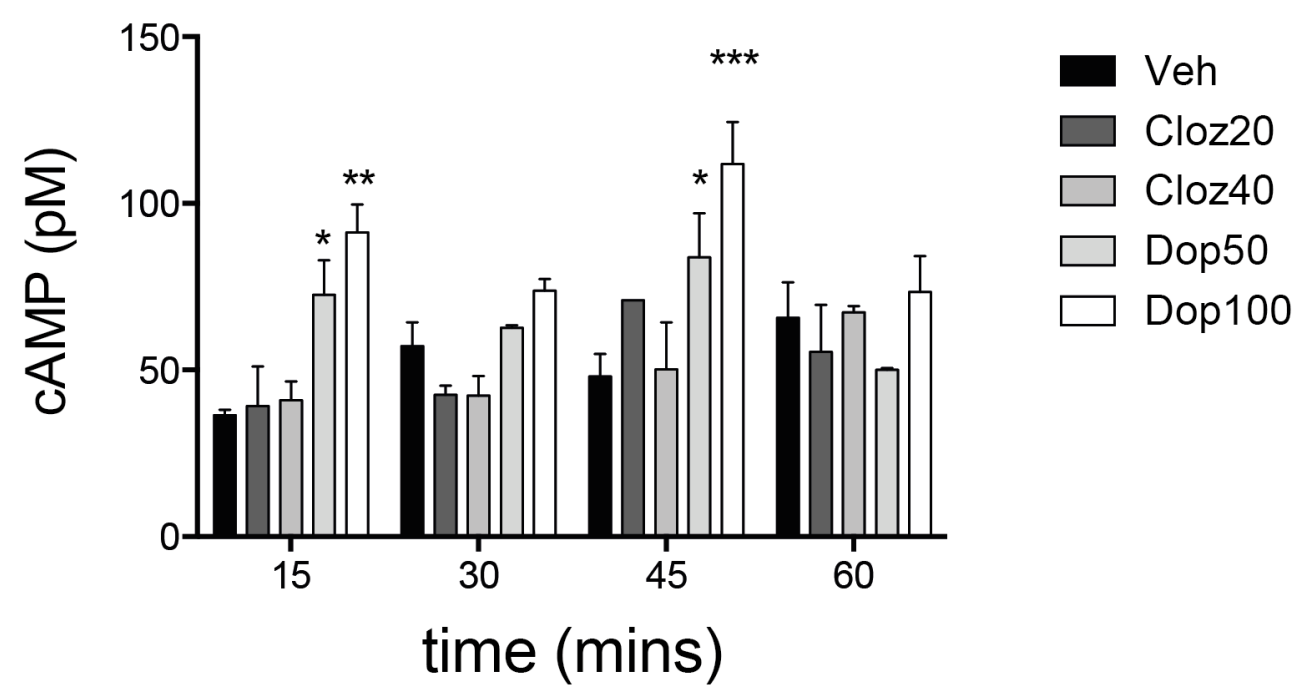

Figure 6.7 Clozapine does not alter cAMP signaling in LPS stimulated macrophages

BMMØ were plated at 100,000 cells/well, were primed with IFN- $\gamma(20 \mathrm{U} / \mathrm{mL})$ overnight and stimulated with lipopolysaccharide for 15,30,45 and 60 minutes in the presence of clozapine (Cloz20; $20 \mu \mathrm{M}, \mathrm{Cloz} 40 ; 40$ $\mu \mathrm{M})$, dopamine (Dop50; $50 \mu \mathrm{M}$. Dop100; $100 \mu \mathrm{M}$ ). Shown are means and SEM of triplicate wells from one representative experiment of two. Statistical analyses performed using a two-way ANOVA and Dunnett's multiple comparisons test. $* \mathrm{p}<0.05, * * \mathrm{p}<0.01, * * * \mathrm{p}<0.001$ and $* * * * \mathrm{p}<0.0001$.

\subsubsection{The Akt-GSK3 pathway modulated LPS-induced cytokine production}

TLR4 activates PI3K and mediates recruitment and phosphorylation of signalling proteins such as Akt, which phosphorylates downstream targets including GSK-3 $\beta$. There is substantial 
evidence that indicates that treatment with clozapine and other atypical antipsychotic agents modify the Akt and GSK-3 $\beta$ signalling pathways (M.-L. Chen et al., 2011; Li et al., 2007; F. Panariello et al., 2012; Roh et al., 2007). Given that this pathway is a key regulator of toll-like receptor signalling (Ko \& Lee, 2016), macrophages were treated with Akt and GSK-3 $\beta$ specific inhibitors to assess the effects of inhibiting Akt or GSK-3 $\beta$ on LPS-induced cytokine secretion. Firstly, to examine the consequence of inhibiting Akt activity on LPS-induced cytokine secretion the Akt1/2 specific inhibitor was added to the culture medium during LPS stimulation for 24 hours; after which IL-12 and IL-10 in the supernatant were measured. In the RAW264.7 macrophage cell line, Akt1/2 inhibitor at $6.25 \mu \mathrm{M}$ augmented the production of IL-12 significantly (Figure 6.8A). In contrast, Akt1/2 inhibitor abolished IL-10 production (Figure 6.8B). These changes in cytokine production occurred at a concentration of Akt1/2 inhibitor that did not affect MTT metabolism (Figure 6.8C), which indicated that cell viability was not impaired. These results confirm previously reported studies that show Akt is able to modulate cytokine production in LPS stimulated macrophages (Ko \& Lee, 2016). 

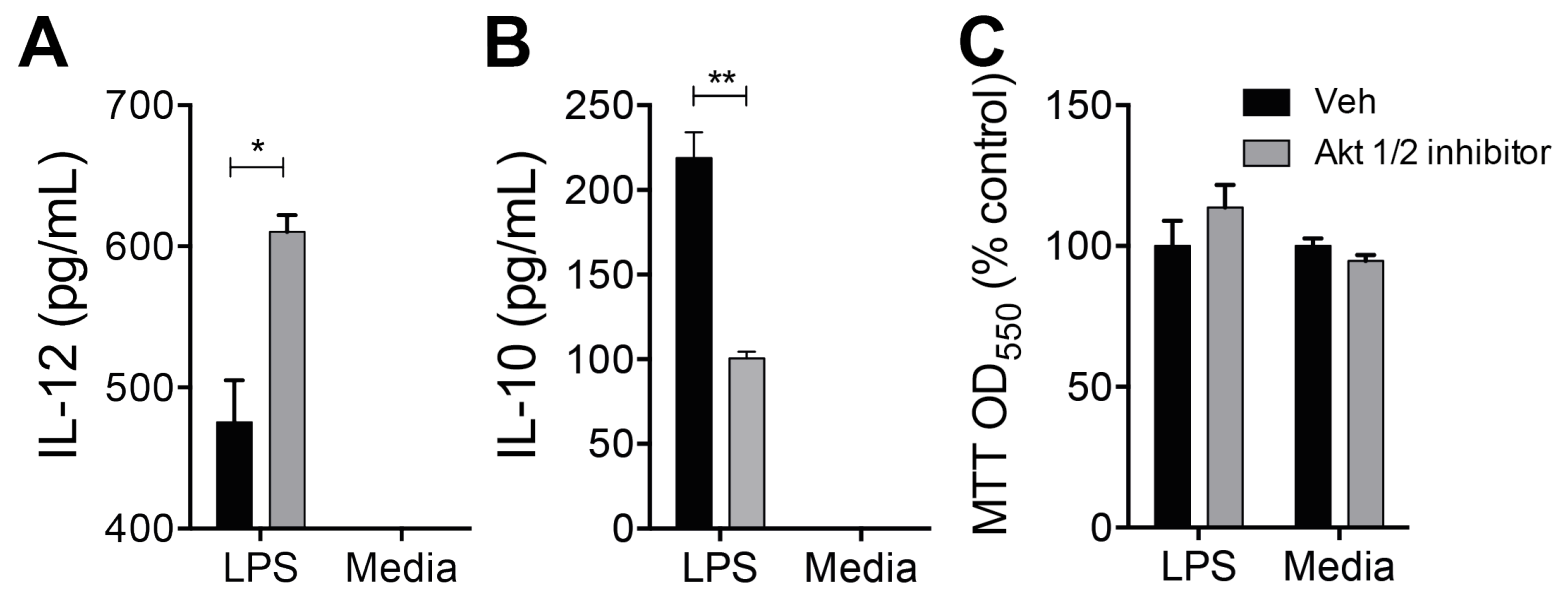

\section{Figure 6.8 Akt1/2 inhibitor increases IL-12 and decreases IL-10 secretion by macrophages}

A-C RAW264.7 cells were plated at 50,000 cells/well, primed with IFN- $\gamma(20 \mathrm{U} / \mathrm{mL})$ and stimulated with lipopolysaccharide for 24 hours with Akt $1 / 2$ inhibitor $(6.25 \mu \mathrm{M})$. A IL-12 and B IL-10 in the supernatants were measured by ELISA. C Cell viability was measured by MTT assay presented as percent of vehicle control. Shown are means and SEM of triplicate wells from one experiment representative of two. Statistical analyses performed using a two-way ANOVA and Dunnett's multiple comparisons test. ${ }^{*} \mathrm{p}<0.05, * * \mathrm{p}<0.01,{ }^{* * *} \mathrm{p}$ $<0.001$ and $* * * * \mathrm{p}<0.0001$.

Interestingly, the Akt1/2 inhibitor was not similarly effective in bone marrow-derived macrophages. At concentrations of $1 \mu \mathrm{M}$ and $3 \mu \mathrm{M}$, a significant reduction in IL-12 in the supernatant is detected after 24 hours (Figure 6.9A). Similarly, a reduction in IL-10 is evident upwards of $1 \mu \mathrm{M}$ of Akt1/2 inhibitor (Figure 6.9B). Akt1/2 inhibitor induced a slight but significant reduction in MTT metabolism from $2 \mu \mathrm{M}$ and continued to decrease in a concentration-dependent manner (Figure 6.9C) and likely explained the inconsistent IL-12 measurements as toxicity was evident at these concentrations. Likewise, the reduction in IL10 in the supernatant from $2 \mu \mathrm{M}$ of Akt1/2 inhibitor (Figure 6.9B) was associated with significant reductions in MTT metabolism (Figure 6.9C), suggesting that loss of IL-10 was due to reduced cell viability. 


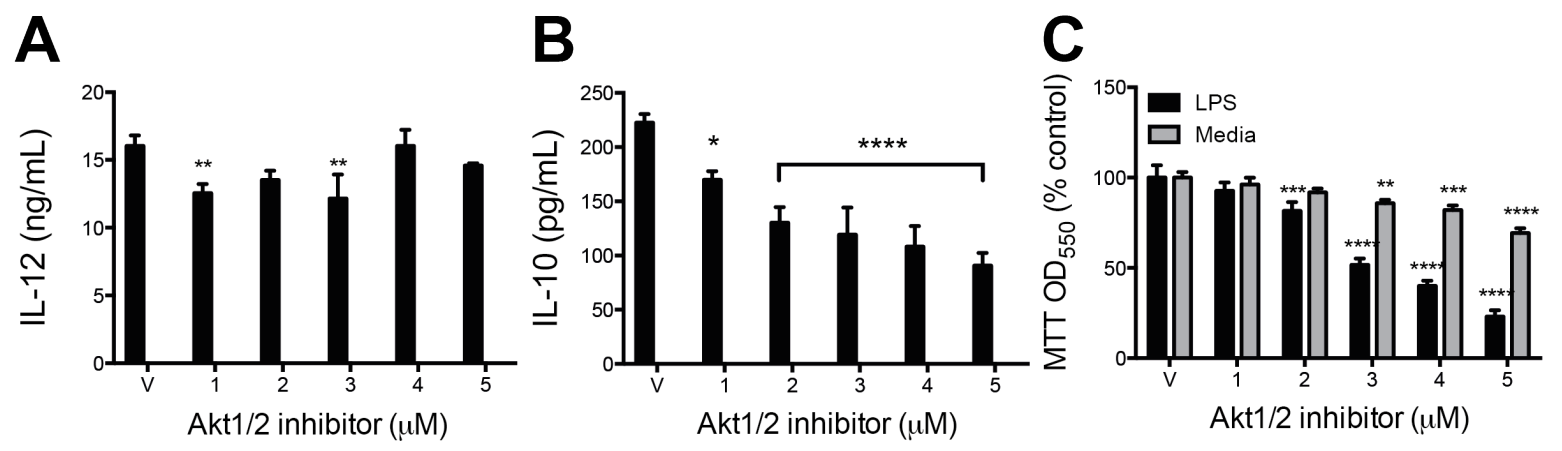

Figure 6.9 Akt1/2 inhibitor is not similarly effective in bone marrow derived macrophages

A-C BMMØ were plated at 100,000 cells/well, were primed with IFN- $\gamma(20 \mathrm{U} / \mathrm{mL})$ and stimulated with lipopolysaccharide for 24 hours in increasing concentrations of Akt1/2 inhibitor. A IL-12 and B IL-10 in the supernatants were measured by ELISA. C Cell viability was measured by MTT assay presented as percent of vehicle control. Shown are means and SEM of triplicate wells from one experiment representative of three. Statistical analyses performed using a two-way ANOVA and Dunnett's multiple comparisons test. * $\mathrm{p}<0.05$, $* * \mathrm{p}<0.01, * * * \mathrm{p}<0.001$ and $* * * * \mathrm{p}<0.0001$

This finding raises the possibility that aberrant Akt signalling in immortalized cell lines may affect LPS induced cytokine production. Alternatively, it is also possible that the concentrations used in these experiments were too high for bone marrow-derived macrophages as it caused significant impairment in MTT metabolism. Accordingly, the experiment was repeated using lower concentrations of Akt1/2 inhibitor. Similarly, Akt1/2 inhibitor decreased IL-12 (Figure 6.10A) and IL-10 (Figure 6.10B) in the supernatant of LPS stimulated macrophages only at concentrations that impaired MTT metabolism (Figure 6.10C), suggesting that the decrease in cytokine secretion is due to cellular death. 


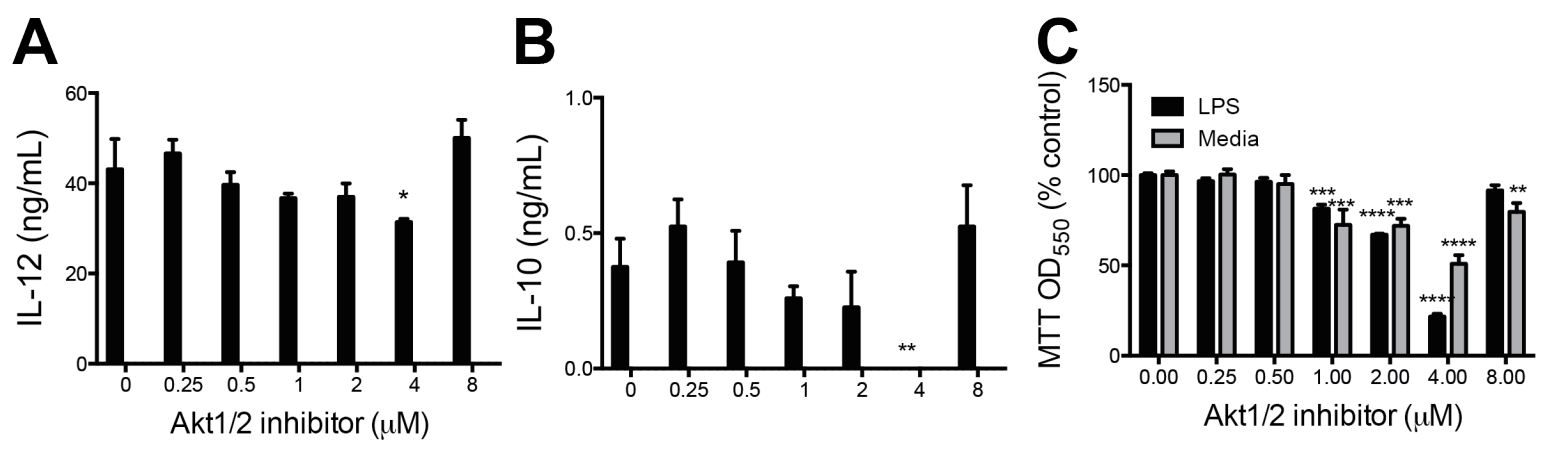

Figure 6.10 Akt1/2 inhibitor is not similarly effective in bone marrow derived macrophages at lower concentrations

A-C BMMØ were plated at 100,000 cells/well, were primed with IFN- $\gamma(20 \mathrm{U} / \mathrm{mL})$ and stimulated with lipopolysaccharide for 24 hours in increasing concentrations of Akt1/2 inhibitor. A IL-12 and B IL-10 in the supernatants were measured by ELISA. C Cell viability was measured by MTT assay presented as percent of vehicle control. Shown are means and SEM of triplicate wells from one experiment representative of two. Statistical analyses performed using a two-way ANOVA and Dunnett's multiple comparisons test. * $\mathrm{p}<0.05$, $* * \mathrm{p}<0.01, * * * \mathrm{p}<0.001$ and $* * * * \mathrm{p}<0.0001$

Activated Akt phosphorylates downstream targets including GSK-3 $\beta$. Phosphorylation of GSK-3 $\beta$ results in inhibition of its constitutively active enzymatic activity. To assess the effect of GSK-3 $\beta$ inhibition directly, a GSK-3 $\beta$ specific inhibitor; CHIR99021 was used. As expected, when CHIR99021 was added to the culture medium, an effect that was opposite to Akt inhibition was observed. Macrophages produced significantly less IL-12 when GSK-3 $\beta$ was inhibited by CHIR99021 (Figure 6.11A). In contrast, IL-10 in the supernatant was significantly increased (Figure 6.11B), and these effects were observed at concentrations that did not affect cell viability (Figure 6.11C). 

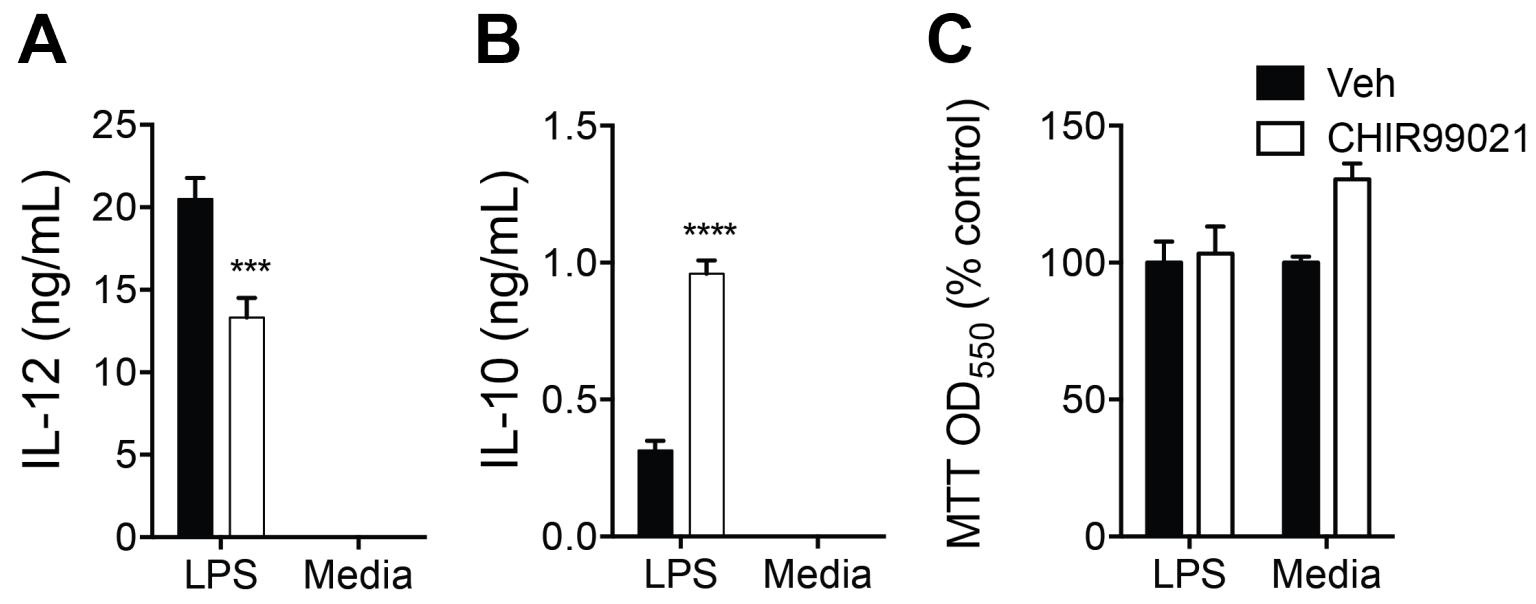

\section{Figure 6.11 CHIR99021 inhibited IL-12 and increased IL-10 secretion in bone marrow derived macrophages}

A-C BMMØ were plated at 100,000 cells/well, were primed with IFN- $\gamma(20 \mathrm{U} / \mathrm{mL})$ and stimulated with lipopolysaccharide for 24 hours in the presence of GSK3 inhibitor (CHIR99021; $1 \mu \mathrm{M})$. IL-12 (A) and IL-10 (B) in the supernatants were measured by ELISA. Cell viability was measured by MTT assay presented as percent of vehicle control (C). Shown are means and SEM of triplicate wells from one experiment. Statistical analyses performed using a two-way ANOVA and Dunnett's multiple comparisons test. * p $<0.05, * * \mathrm{p}<$ $0.01, * * * \mathrm{p}<0.001$ and $* * * * \mathrm{p}<0.0001$.

Interestingly, the CHIR99021 was not similarly effective in the RAW 264.7 cell line. Unlike in the bone marrow-derived macrophages, CHIR99021 decreased IL-12 (Figure 6.12A) and IL-10 (Figure 6.12B) production by LPS-stimulated macrophages at low concentrations of 1 $\mu \mathrm{M}$. However at $5 \mu \mathrm{M}$, IL-12 production was increased while IL-10 continually decreased. At these concentrations, up to $5 \mu \mathrm{M}$ of CHIR99021 did not cause any alteration in MTT metabolism (Figure 6.12C) suggesting that cell viability was maintained. These results are inconsistent with findings observed in primary bone marrow-derived macrophages. 


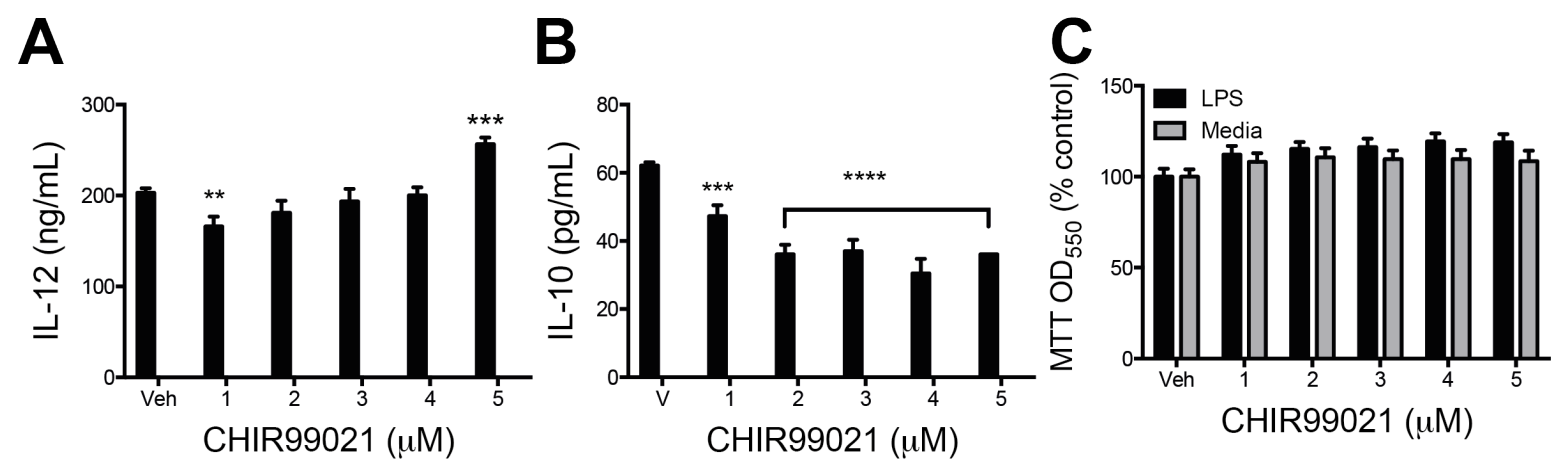

Figure 6.12 CHIR99021 is not similarly effective in the RAW 264.7 cell line

A-C RAW264.7 cells were plated at 50,000 cells/well, primed with IFN- $\gamma(20 \mathrm{U} / \mathrm{mL})$ and stimulated with lipopolysaccharide for 24 hours in increasing concentrations of CHIR99021. A IL-12 and B IL-10 in the supernatants were measured by ELISA. C Cell viability was measured by MTT assay and presented as percent of vehicle control. Shown are means and SEM of triplicate wells from one experiment representative of two. Statistical analyses performed using a two-way ANOVA and Dunnett's multiple comparisons test. ${ }^{*} \mathrm{p}<0.05$, $* * \mathrm{p}<0.01, * * * \mathrm{p}<0.001$ and $* * * * \mathrm{p}<0.0001$.

\subsubsection{Clozapine did not alter LPS-induced phosphorylation of signaling pathways}

It is clear that modifying the activation of signalling enzymes has profound effects on the cytokine secretion of LPS-stimulated macrophages. To understand how clozapine is able to alter the cytokine production of LPS-stimulated macrophages so that they produce less IL-12 and more IL-10, multiple signalling networks were investigated by measuring phosphorylated and total protein levels of Akt, p70S6K, p38, JNK, ERK, CREB, STAT3, STAT5, GSK-3 $\beta$ and NFKB p65 using a bead-based multiplex assay in a time resolved manner up to one-hour post-LPS. The multiplex assay allowed measurement of multiple cell signalling molecules from the same sample for accurate comparison of multiple pathways. These proteins were chosen as they represented multiple pathways that could be modulated by G-protein coupled receptors and are components of LPS signalling. Total protein levels of CREB (Figure 6.13A), JNK (Figure 6.13B), p38 (Figure 6.13C), ERK (Figure 6.13D), Akt (Figure 6.13E), STAT3 (Figure 6.13F), STAT5 (Figure 6.13G), GSK-3 $\beta$ (Figure 6.13H) and NFkB p65 (Figure 6.13I) were not significantly affected by LPS stimulation up to 1-hour post stimulation. 


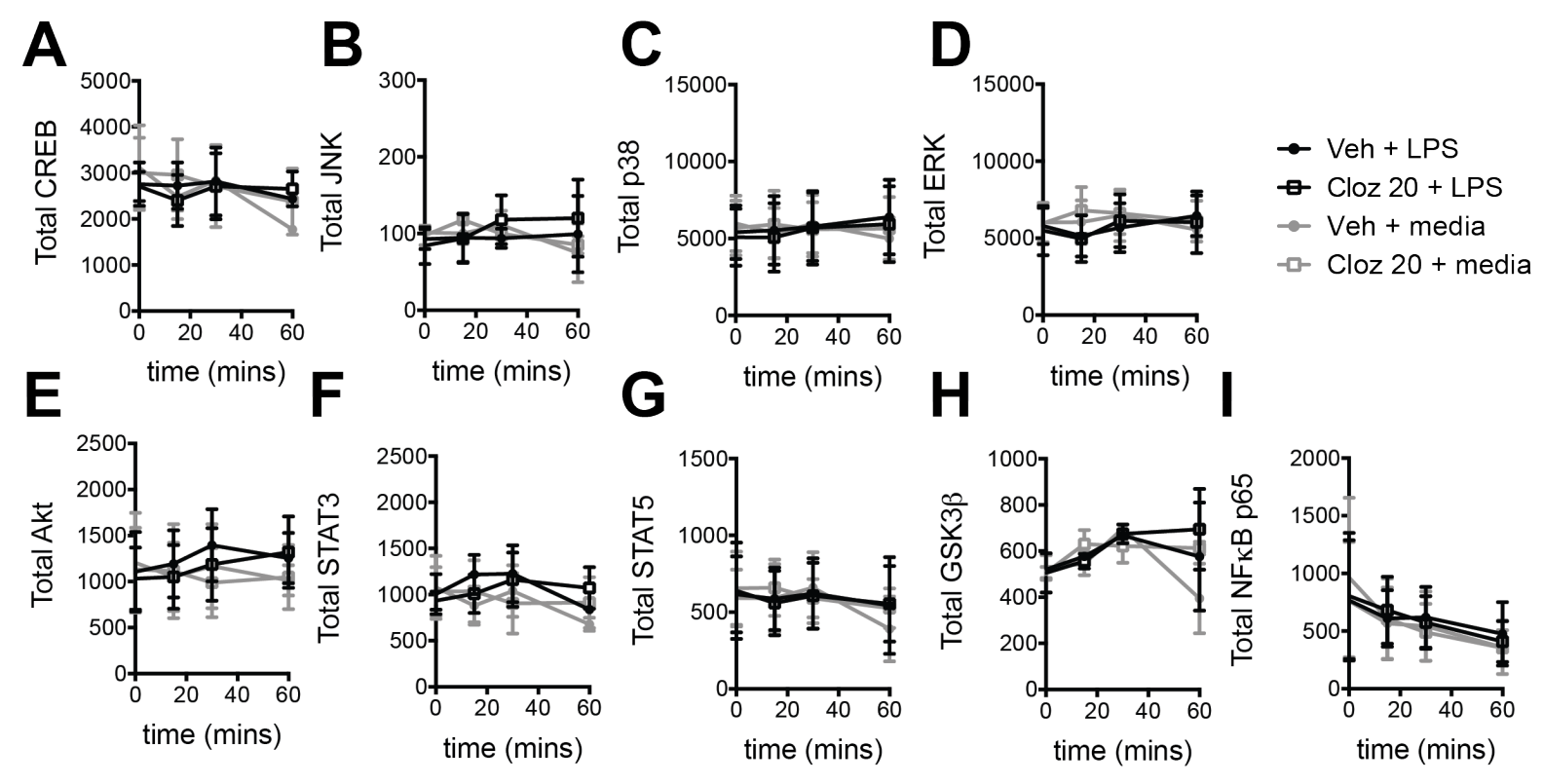

Figure 6.13 Clozapine does not alter total protein levels of several signaling proteins in response to lipopolysaccharide

A-I BMMØ were plated at 100,000 cells/well, were primed with IFN- $\gamma(20 \mathrm{U} / \mathrm{mL})$ and stimulated with LPS for $0,15,30$ or 60 minutes in the presence of clozapine (Cloz 20;20 $\mu \mathrm{M})$. Presented are the total protein measurements for A CREB, B JNK, C p38, D ERK, E Akt, F STAT3, G STAT5A/B, H GSK3 $\beta$ and I NFKB p65 at each time point. Shown are means and SEM of two independent experiments combined. Statistical analyses by two-way ANOVA.

LPS stimulation induced phosphorylation of CREB (S133; Figure 6.14A), JNK (T183/Y185; Figure 6.14B), p38 (T180/Y182; Figure 6.14C), ERK (T185/Y187; Figure 6.14D), Akt (S473; Figure 6.14E), STAT3 (S727; Figure 6.14F) and GSK-3 $\beta$ (S9; Figure 6.14H). No significant phosphorylation of STAT5A/B (Y694/Y699) was detected after one hour of stimulation. (Figure 6.14G). Phosphorylation of NFKB p65 (s536) was not detected after one hour of LPS (Figure 6.14I) stimulation presumably because the NFKB p65 detection reagents were not validated for mouse samples.

Phosphorylation of p38 (Figure 6.14C), JNK (Figure 6.14B) and ERK (Figure 6.14D) phosphorylation in response to LPS was immediate and peaked after only 15 minutes of LPS stimulation whereas CREB (Figure 6.14A) and STAT3 (Figure 6.14F) phosphorylation peaked after 30 minutes. Akt and GSK $3 \beta$ peaked after 1 hour. Clozapine was added to the culture medium at a concentration of $20 \mu \mathrm{M}$. This concentration was chosen for the timeresolved phosphorylation studies since at this concentration IL-12 secretion is reduced and IL10 is increased without affecting viability. Higher concentrations of clozapine were avoided to 
minimize artefacts in phosphorylation due to the toxicity of clozapine in vitro. Uneventfully, clozapine did not affect significantly LPS-induced phosphorylation of the signalling proteins investigated in this study (Figure 6.14).
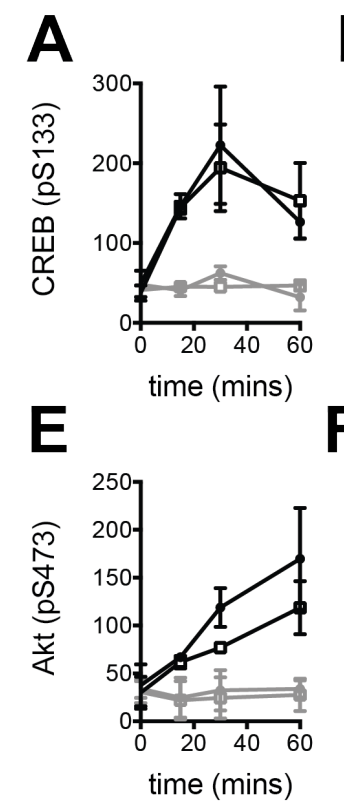

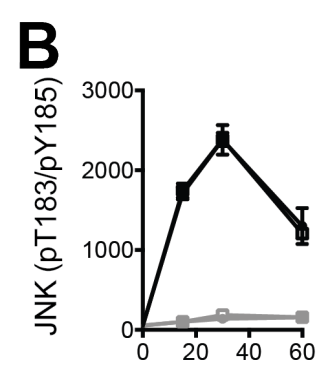

$\mathbf{F}$

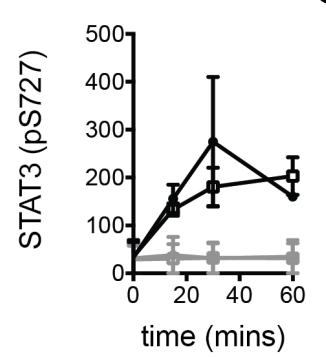

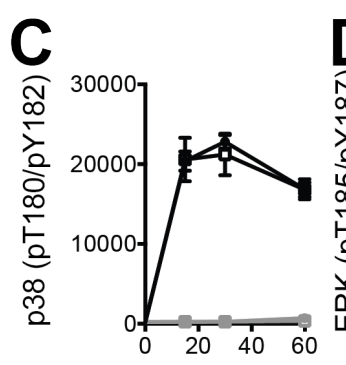

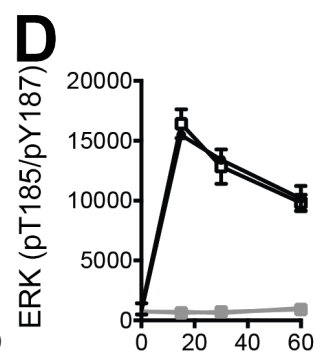

$\rightarrow$ Veh + LPS

๑ Cloz $20+$ LPS

$\rightarrow$ Veh + media

$-\square-$ Cloz $20+$ media
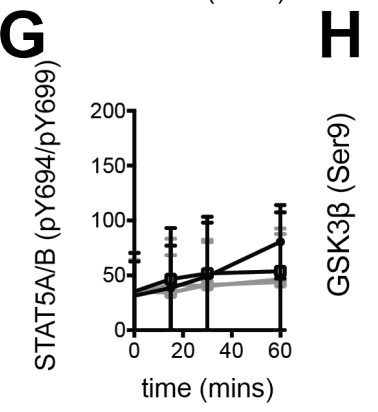

\author{
年
}


alterations in the phosphorylation of these cell signalling pathways up to one hour after LPS stimulation.
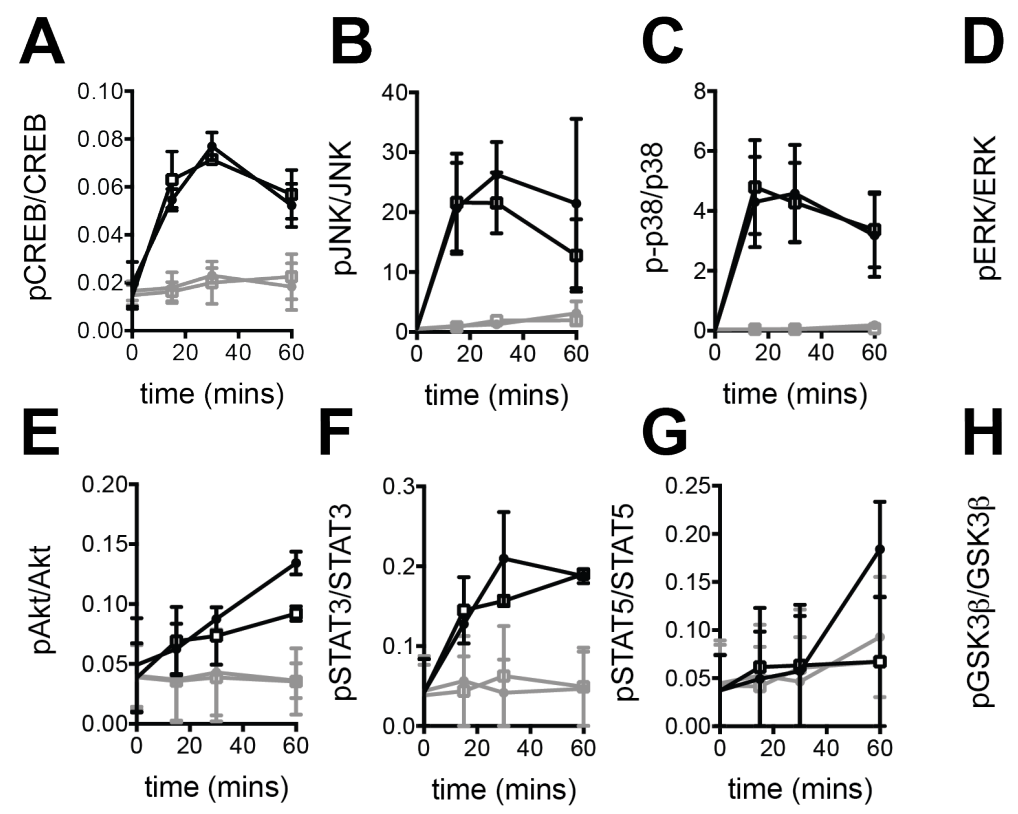
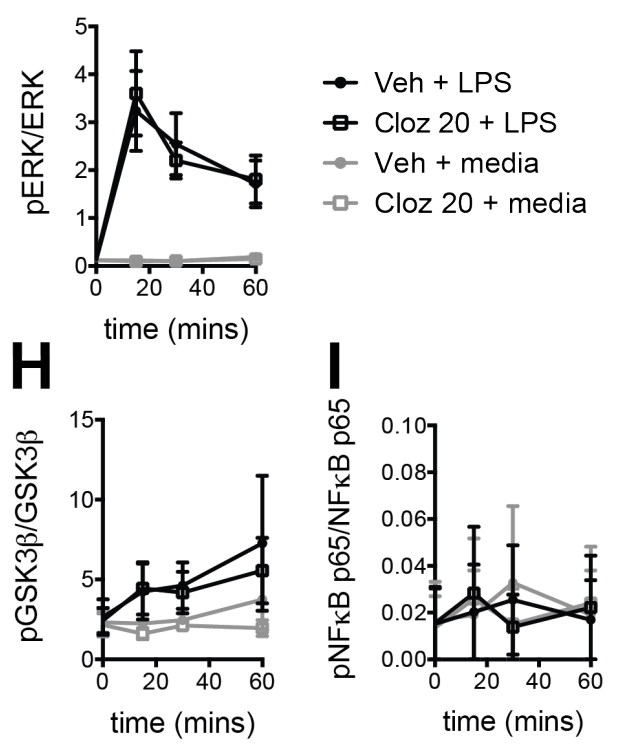

\section{Figure 6.15 Clozapine does not alter LPS-induced phosphorylation of signaling proteins}

A-I BMMØ were plated at 100,000 cells/well, were primed with IFN- $\gamma(20 \mathrm{U} / \mathrm{mL})$ and stimulated with lipopolysaccharide for $0,15,30$ or 60 minutes in the presence of clozapine (Cloz 20;20 $\mu \mathrm{M}$ ). Presented are the ratio of phosphorylated/total protein for CREB (pS133; A), JNK (pT183/pY185; B), p38 (pT180/pY182; C), ERK (pT185/pY187; D), Akt (pS473; E), STAT3 (pS727; F), STAT5A/B (pY694/699; G), GSK-3ß (pS9; H) and NFKB p65 (s536; I) at each time point. Shown are means and SEM of two independent experiments combined. Statistical analyses by two-way ANOVA.

No positive signal was detected for phosphorylated and total p70S6K using the Milliplex multipathway cell signalling kit for any of the experiments conducted. Since the NFKB p65 detection reagents included in the Milliplex multi-pathway cell signalling kit was not validated for mouse samples, phosphorylated and total NFKB p65 was measured in cell lysates by ELISA. After 30 minutes or 60 minutes of LPS stimulation, NFKB p65 is phosphorylated at s536 and is not affected by clozapine at $20 \mu \mathrm{M}$ or $40 \mu \mathrm{M}$. Similarly, risperidone at $40 \mu \mathrm{M}$ did not affect NFKB p65 phosphorylation (Figure 6.16A). Neither clozapine nor risperidone had any effect on NFкB p65 phosphorylation in unstimulated wells (Figure 6.16A). Total NFкB p65 was unaffected by clozapine, risperidone or LPS (Figure 6.16B). Similarly, when data were presented as the ratio of phosphorylation/total, LPS stimulated the phosphorylation of NFKB 
p65 but was unaffected by clozapine or risperidone (Figure 6.16C). These results indicate that clozapine did not alter the cytokine production of macrophages by altering NFאB p65 signalling in the first hour of LPS stimulation. 

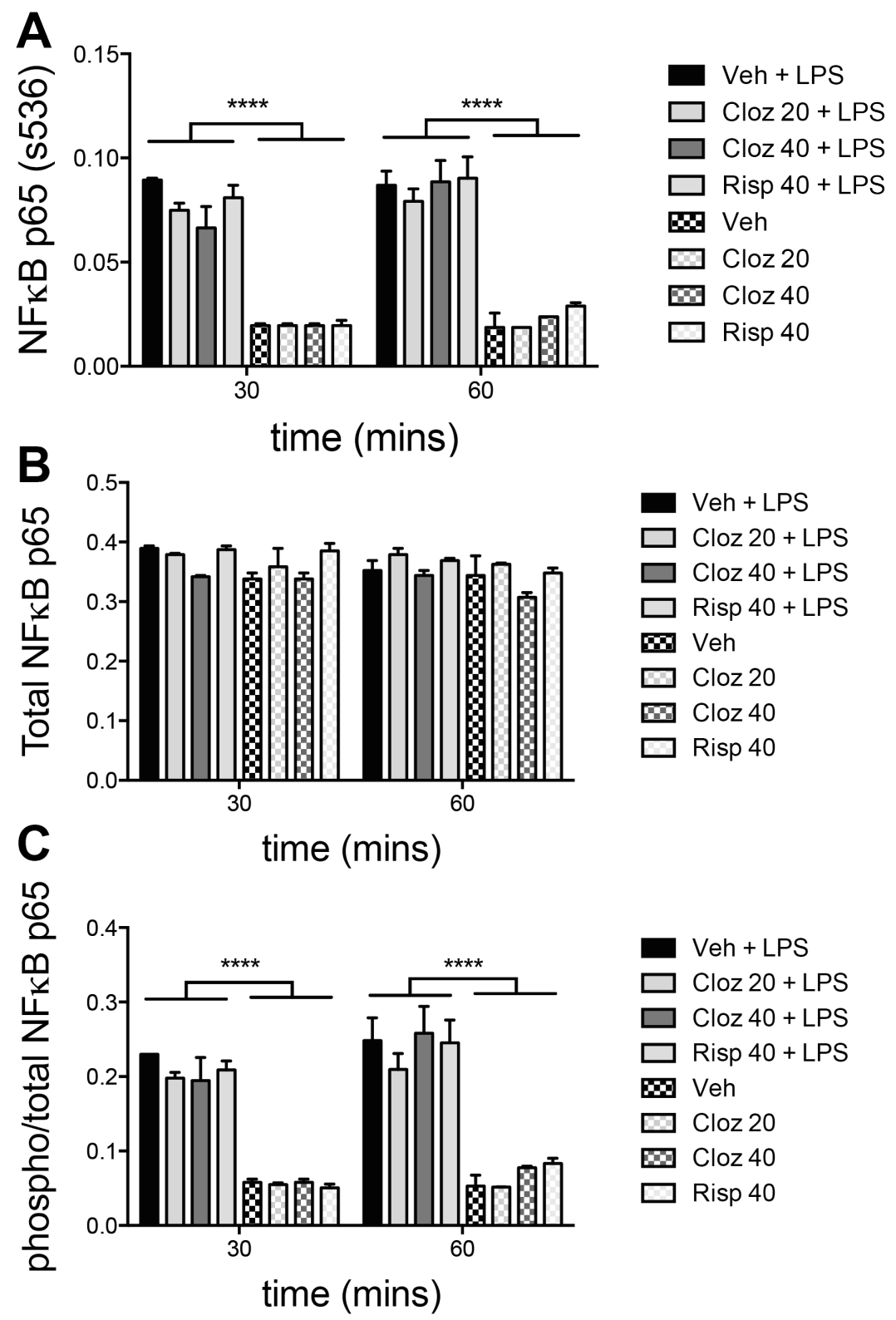

\section{Figure 6.16 Phosphorylation of NFKB p65 at s536 is not affected by clozapine}

A-C BMMØ were plated at 100,000 cells/well, were primed with IFN- $\gamma(20 \mathrm{U} / \mathrm{mL})$ and stimulated with lipopolysaccharide for 30 or 60 minutes in the presence of clozapine (Cloz 20;20 $\mu \mathrm{M}, \mathrm{Cloz} 40 ; 40 \mu \mathrm{M})$ or risperidone (Risp 40; $40 \mu \mathrm{M}$ ). A Absorbance at $450 \mathrm{~nm}$ of phosphorylated NFKB p65 (s536) was measured by ELISA. B Absorbance at $450 \mathrm{~nm}$ of total NFKB p65 was measured by ELISA. C Ratio of phosphorylated/total NFKB p65 in matched wells. Shown are means and SEM of duplicate wells from one experiment. Statistical analyses were performed by two-way ANOVA and Tukey's multiple comparison test. $* * * * p<0.0001$. 


\subsection{Discussion}

Our laboratory has shown that atypical antipsychotic agents are able to alter the activation of macrophages and microglia. Although recent studies have previously identified that clozapine has immune modulating properties, there is very little published evidence on the mechanism by which clozapine is able to modulate the immune response. This chapter aimed to investigate how clozapine is able to alter the activation of macrophages and microglia as a potential mechanism by which they are effective at reducing disease during EAE.

In EAE and MS, macrophages and microglia are in an inflammatory state of activation supported by IFN- $\gamma$ from Th1 biased T cells. To model this situation in vitro, bone-marrow derived macrophages were first primed with IFN- $\gamma$ followed by stimulation with LPS resulting in high production of IL-12 and some IL-10 that is characteristic of classical macrophage activation. We show here that clozapine as well as risperidone was able to alter the activation of macrophages towards a less inflammatory phenotype by producing less IL-12 and more IL10. It is interesting to note that dopamine itself altered activation of macrophages towards a less inflammatory state in a similar manner, given that clozapine is an antagonist of dopamine receptors. We have previously shown that this effect cannot be replicated when specifically antagonizing D1R, D2R or both, indicating that clozapine must also act on other receptors like the serotonin, histamine, adrenergic and muscarinic receptor families to induce the antiinflammatory response. The fact that clozapine has such a wide receptor binding profile makes it a challenging task to decipher the precise mechanism experimentally and as a compromise of finding the precise receptors involved, an approach to measure changes in intracellular signalling was implemented instead.

There is evidence to suggest that clozapine may alter cAMP signalling pathways. Firstly, clozapine is known to have partial agonist activity at several GPCR, and these are typically coupled to cAMP signalling. It has recently been shown that clozapine can inhibit cAMP accumulation in CHO cells transfected with the $\mathrm{m} 2$ receptor (Olianas, Maullu, \& Onali, 1999). It was also shown in 2003 by Pozzi et al. that intraperitoneal injection of clozapine reduced the phosphorylation of the cAMP response element-binding protein (CREB) whereas injection of haloperidol increased CREB phosphorylation in mice (Pozzi et al., 2003). These studies indicate some potential for clozapine to alter cAMP signalling in other cells such as macrophages and microglia in the CNS. 
Recognizing that cAMP signalling affects the cytokine production of macrophages stimulated with LPS, it was shown in these experiments that clozapine does not affect the accumulation of cAMP during the first hour of LPS stimulation when transcription of cytokines has occurred (Sharif, Bolshakov, Raines, Newham, \& Perkins, 2007), indicating that the anti-inflammatory effect observed with clozapine in this model is independent of cAMP signalling.

A substantial number of studies have demonstrated the potential for clozapine and other atypical antipsychotic agents to modify the Akt-GSK-3 $\beta$ pathway (Li et al., 2007; Roh et al., 2007) and the inhibition of GSK-3 $\beta$ is believed by some investigators to be a mechanism by which atypical antipsychotic agents are therapeutic during psychiatric illness (Cole, 2013; Jope $\&$ Roh, 2006). The Akt-GSK3 $\beta$ was therefore investigated in this study. Firstly, the experiments presented in this chapter showed that there are discrepancies between the effects of Akt1/2 inhibitor and the GSK-3 $\beta$ inhibitor CHIR99021 on RAW 264.7 and primary macrophages. Although insufficient experimental data is available to elucidate these differences, a possible explanation for this discrepancy is that cancerous cell lines such as RAW 264.7 in general are constitutively high in Akt activity to promote survival and proliferation (Testa \& Bellacosa, 2001) and may not be an ideal model for studies in phosphoprotein signalling.

Nevertheless, clozapine has previously been shown to alter signalling pathways including Akt and GSK3, which are known to alter toll-like receptor initiated cytokine production. Indeed, when Akt1 and Akt2 activity is inhibited to activate glycogen synthase kinase-3 downstream, the production of IL-10 is suppressed while IL-12 is increased in LPS stimulated macrophages. In contrast, when glycogen synthase kinase-3 activity is inhibited the secretion of IL-10 is increased and IL-12 is inhibited. This is a mechanism that has been previously published (Martin, Rehani, Jope, \& Michalek, 2005) and has an effect that is similar to the effect we see when we treat macrophages with risperidone or clozapine, albeit much more pronounced. Indicating that if clozapine were able to modulate GSK-3 $\beta$, we would observe this effect. It was therefore hypothesized that clozapine was able to suppress IL-12 and increase IL-10 in macrophages by its reported ability to modulate GSK-3 $\beta$ as it had similar effects to the GSK$3 \beta$ inhibitor. Multiple cell signalling pathways were analysed in time course experiments up to 1 hour after LPS stimulation and these did not detect any effect by clozapine on the 
phosphorylation of GSK-3 $\beta$ or other cell signalling proteins investigated. Given that no significant alterations in cell-signalling proteins were detected during the early signalling events it may suggest that clozapine has an effect on other pathways of protein secretion.

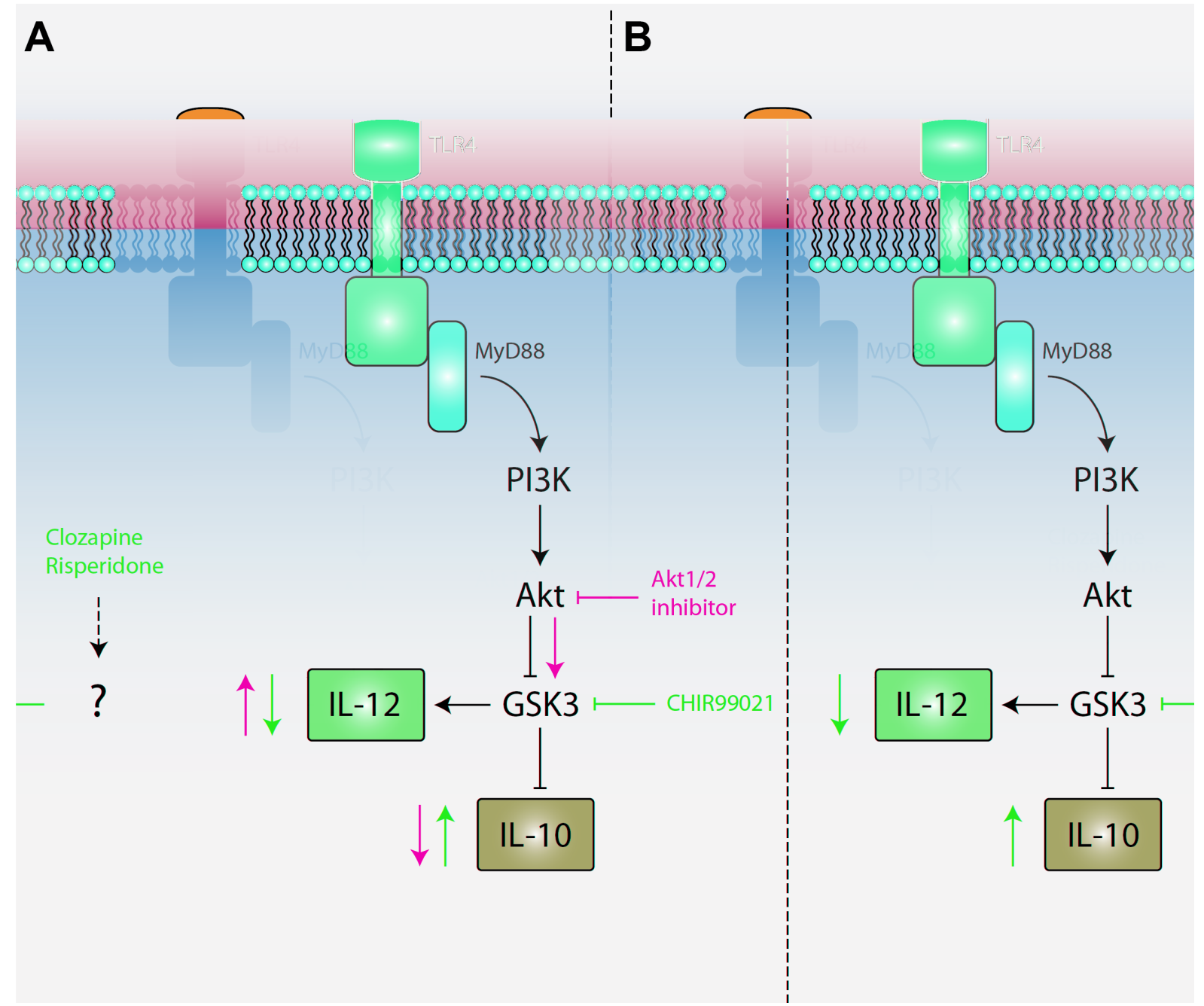

\section{Figure 6.17 Potential mechanism by which clozapine is able to reduce IL-12 and increase IL-10}

A During LPS stimulation, TLR4 signaling activates PI3K through a MyD88 dependent pathway which activates Akt downstream. Akt activation inhibits GSK3 which regulates IL-12 and IL-10 production. When GSK3 is inhibited using a specific inhibitor, CHIR99021 (shown in red), IL-12 production is supressed while IL-10 is increased. In contrast, when an Akt inhibitor is used, Akt1/2 inhibitor inhibits Akt which activates GSK3 downstream and results in an opposite effect that is evident with increased IL-12 production and decreased IL-10. B Clozapine and risperidone could through an unidentified mechanism inhibit GSK3 and result in reduced IL-12 production and increased IL-10 similar to the effect of CHIR99021 in A. 
Although the GSK-3 $\beta$ pathway is a promising target by which clozapine may alter the activation of macrophages, other pathways including the mTOR pathway also have potential to be modulated by clozapine. Like GSK-3 $\beta$, mTOR has a significant role in regulating LPS induced cytokine production. Activating mTOR suppresses IL-12 production and increases IL10 in LPS stimulated macrophages (Weichhart et al., 2008). Currently, there are no published reports describing the effects of clozapine on the mTOR pathway, although the structurally similar atypical antipsychotic olanzapine has been shown to activate mTOR in mice liver (Schmidt et al., 2013). Therefore, it is possible that clozapine affects both mTOR and GSK-3 $\beta$ signalling pathways to modify innate immune responses.

\subsection{Conclusions}

The experiments in this chapter show that Akt and GSK-3 $\beta$ are important signalling enzymes for regulating the secretion of pro and anti-inflammatory cytokines in macrophages. While atypical antipsychotic agents have been described to alter the Akt-GSK-3 $\beta$ pathway, the experiments conducted did not measure changes in LPS induced Akt or GSK-3 $\beta$ phosphorylation in the presence of clozapine. In addition, no alteration in the phosphorylation of CREB, JNK, p38, ERK, STAT3, STAT5 and NFKB p65 was detected and may indicate that clozapine has effects on other cell signalling pathways not investigated here or has effects downstream of phosphoprotein signalling. 
Chapter 7: Investigation into novel therapeutic strategies for multiple sclerosis 


\subsection{Introduction}

The previous chapters have demonstrated the efficacy of using clozapine to reduce disease in EAE, an animal model commonly used for studying MS. MS is a debilitating disease that affects around 2.5 million people around the world with a diverse course of disease that present with symptoms ranging from mild cognitive disturbance through to severe physical disability in the progressive forms of disease, placing significant strain on patient quality of life and cost of healthcare. Patients with progressive forms of disease currently have no approved treatments available (Gajofatto \& Benedetti, 2015), leaving them only to continually accumulate disability. Thus, there is an urgent need for novel therapeutic strategies to improve the quality of life and halt disease progression in these patients. While clozapine or risperidone alone show promise, there is also the possibility that their protective effects could be augmented by complementing their therapeutic pathways with an alternative therapeutic agent, and this chapter explores this possibility.

\subsubsection{L-DOPA and clozapine as potential therapeutics for multiple sclerosis}

A considerable number of studies have described the influence of psychiatric illness such as depression and other behavioural on immune homeostasis. Many of the effects of psychosocial stressors on the immune profile have been attributed to the action of catecholamines, which are released via activation of the sympathetic nervous system (i.e. the "fight or flight" response). Specifically, peripheral blood mononuclear cells (PBMC) from MS patients who scored higher in the Perceived Stress Scale generally produced more inflammatory cytokines when compared to healthy controls (Sorenson, Janusek, \& Mathews, 2013). Many studies have identified that increased stress generally correlates with increased risk for relapse during MS (Karagkouni, Alevizos, \& Theoharides, 2013) and the use of coping strategies for stress negatively correlates with the severity of disease symptoms (Somer et al., 2010). In addition to the impact of psychological stress, the influence of the sympathetic nervous system in regulating inflammatory responses is exemplified by a study, which shows that voluntary wheel running in mice delays the onset of EAE (Benson et al., 2015).

A recent review by Pacheco et al. in 2014 suggests that autoimmune disorders such as multiple sclerosis are associated with dysregulated dopamine homeostasis (Rodrigo Pacheco, Contreras, $\&$ Zouali, 2014). Of note, the catecholamine dopamine inhibits the production of IFN- $\gamma$ and 
IL-17A in both healthy and MS patient PBMC (Melnikov, Belousova, Murugin, Pashenkov, \& Воуко, 2016), and we have shown in the previous chapters that dopamine receptors are expressed on cells of the immune system including macrophages, monocytes and lymphocytes indicating that these immune cells can directly respond to dopamine. Furthermore, we have previously published experimental data demonstrating that dopamine suppresses the production of IL-12 and increases IL-10 by LPS-stimulated macrophages (D. O'Sullivan et al., 2014). Interestingly, when clozapine is added together with dopamine during LPS stimulation of macrophages there is a marked decrease in IL-12 production that is greater than with either individual agent (unpublished from honours thesis, Zareie P 2013) and suggests that dopamine and clozapine together may have an additive effect in reducing inflammatory processes. This chapter aims to investigate this effect during EAE by increasing the available dopamine in the presence of clozapine as an effective therapeutic for multiple sclerosis.

\subsubsection{Risperidone and fluoxetine as potential treatments for multiple sclerosis}

Since other psychiatric drugs have recently been shown to have immune modifying effects, the potential for re-purposing therapeutic agents used for psychiatric illnesses to treat MS must be investigated. Depression is one of the most common psychiatric illness in the modern world and it is increasingly evident that depression is associated with chronic inflammation in the CNS.

Historically, it was believed that depression arose as an imbalance of neurotransmitters in the brain. Firstly, norepinephrine and then later studies suggested that a serotonin imbalance was responsible for depression. A commonly used therapeutic agent for treating psychiatric illnesses such as major depression is fluoxetine, a selective serotonin re-uptake inhibitor (SSRI) that increases pre-synaptic concentrations of serotonin. The mechanism of SSRI in treating depression was previously understood to correct serotonin deficiencies in affected patients; however, no conclusive evidence has ever been published that demonstrates serotonin deficiencies in patients with depression compared to healthy volunteers. Animal studies show that serotonin-deficient mice did not develop behavioural abnormalities (such as depression like symptoms) when compared to wild type mice. Additionally, high doses of the serotonin precursor L-tryptophan was not effective at treating depression symptoms (Mendels, Stinnett, Burns, \& Frazer, 1975), adding considerable doubt to the serotonin deficiency theory of depression. Instead, experimental data has more convincingly shown that depression normally coincides with clinical signs of an inflammatory response and shares a common feature of 
"sickness behaviour" during a fever response to infection that is normally characterized by lethargy, depression and anxiety. Is depression an inflammatory disorder? At this time, there are no definitive studies to make conclusions; however, there is evidence that has since demonstrated the anti-inflammatory effects of drugs used to treat psychiatric illnesses including fluoxetine for depression. Fluoxetine is known to partially rescue the immune changes induced by an auditory stress signal, as well as effectively limiting pathology during collagen-induced arthritis in mice (Sacre et al., 2010). Moreover, fluoxetine inhibits lipopolysaccharide (LPS) induced secretion of TNF- $\alpha$, IL-1 $\beta$, NO and ROS by microglia and protects against LPS-induced neuronal loss indicating that fluoxetine is able to inhibit microglial activation similarly to clozapine or risperidone (Zhang et al., 2012). While no conclusive evidence has been published that describes the mechanism by which SSRI like fluoxetine are able to alter the immune response or mood disorders, many studies have attempted to do so and have since shown that fluoxetine can act in a manner similar to other psychiatric drugs that modulate the Akt-GSK3 pathway. This pathway is already understood to modulate mood disorders and the immune response during EAE. Moreover, fluoxetine, which inhibits GSK3, has previously been shown to reduce disease during chronic EAE in rats (Yuan et al., 2012) and has been identified as a potential therapeutic for multiple sclerosis in a recent systematic review (Vesterinen et al., 2015). In a similar manner to clozapine and risperidone, fluoxetine treatment increases phosphorylation of GSK3 in the brains of mice, and this effect may be responsible for the reduction in EAE disease given that inhibition of GSK3 by specific inhibitors can independently reduce EAE severity (Beurel et al., 2013). Accordingly, if GSK3 is the mechanism by which EAE is suppressed by psychiatric drugs then modulating and inhibiting GSK3 activity more effectively by combining two treatments may equate to an improvement in therapeutic response. In support of this idea, risperidone treatment improves the anti-depressant effects of fluoxetine and is often co-prescribed for treatment resistant depression in the clinic. Animal studies have shown that co-administration of both fluoxetine and risperidone together can increase GSK3 phosphorylation in the mouse brain much more than with either agent alone, causing an improvement in depression symptoms and augmenting immune modulating properties by further altering macrophages to become less inflammatory (Roman, Kusmierczyk, Klimek, Rogoz, \& Nalepa, 2012). These results suggest that risperidone and fluoxetine co-administered may improve therapeutic efficacy in EAE.

In light of the studies discussed, two questions present themselves. Does the co-administration of L-DOPA and clozapine or risperidone and fluoxetine improve the efficacy of either 
clozapine or risperidone alone in treating EAE? This chapter presents experimental in vivo data assessing the effects of L-DOPA, clozapine, risperidone and fluoxetine during EAE to assess whether these agents have the potential to synergistically improve therapy in a mouse model of MS.

\subsection{Aims}

The aim of this chapter is to test hypotheses based on previous work from our laboratory as well as previously published research to develop novel therapeutic regimes for the treatment of multiple sclerosis using EAE as an animal model. These therapeutic regimes are focused on re-purposing already available therapeutic agents with anticipation of translating these findings for rapid clinical use.

\subsubsection{Specific Aims}

1. To test whether increasing dopamine levels in the CNS using Levodopa/Carbidopa during clozapine treatment is more effective at reducing EAE disease than clozapine alone.

2. To test whether risperidone and fluoxetine administered together can improve therapeutic efficacy of either agent alone during EAE. 


\subsection{Results}

\subsubsection{Levodopa/Carbidopa treatment does not alter the disease course of EAE}

Currently, there are no studies evaluating the effects of L-DOPA treatment during EAE. To determine whether exogenous dopamine in the CNS can alter the disease course of EAE, mice were treated initially with increasing doses of Sinemet ${ }^{\circledR}$ (4:1 L-DOPA/Carbidopa; L-DOPA) dissolved in their drinking water one day prior to the induction of EAE. Mice presented with typical EAE in all cases and none of the experimental groups presented with altered EAE disease progression (Figure 7.1A). EAE mice began to lose weight at the same time as the onset of disease. All doses of L-DOPA did not significantly affect the weight loss of mice when compared to vehicle treated mice (Figure 7.1B). All experimental groups receiving increasing doses of L-DOPA succumbed to disease at a similar stage (Figure 7.1C) and reached a similar peak disease score to untreated mice (Figure 7.1D) equating to similar overall disease burdens in all experimental groups (Figure 7.1E) indicating that L-DOPA at the doses expected to increase CNS dopamine did not have any significant effect on EAE disease progression. 

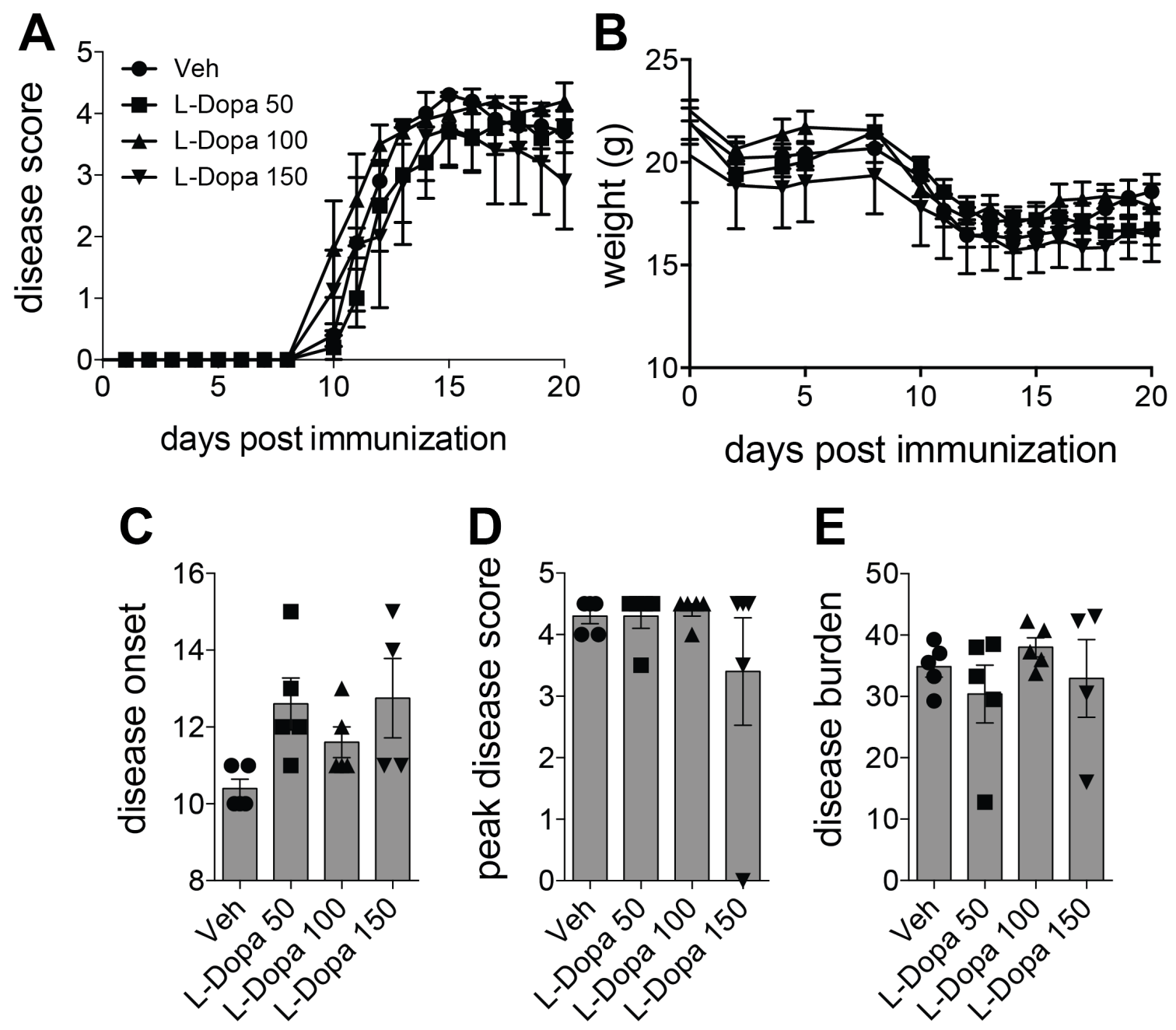

\section{Figure 7.1 Levodopa/Carbidopa does not alter EAE disease progression}

A-E C57BL/6J mice were treated with indicated concentrations of L-DOPA/Carbidopa (L-Dopa 50 = 50/12.5 $\mathrm{mg} / \mathrm{kg} /$ day; $100=100 / 25 \mathrm{mg} / \mathrm{kg} /$ day $; 150=150 / 37.5 \mathrm{mg} / \mathrm{kg} /$ day) in their drinking water one day prior to EAE induction. A Mice were scored daily for disease (0; normal - 5; moribund) and $\mathbf{B}$ weighed to monitor overall health. C Disease onset mice with EAE only (d.p.i) D Maximum disease score reached (d.p.i). E Disease burden (AUC). Shown are means and SEM of individual mice from one experiment $(\mathrm{n}=5$ mice in each group). Statistical analyses by one-way ANOVA and Dunnett's multiple comparison test. 


\subsubsection{L-DOPA does not enhance clozapine protection during EAE}

To assess whether exogenous dopamine can work in synergy with clozapine to reduce EAE disease in vivo, mice were co-treated with L-DOPA $(100 \mathrm{mg} / \mathrm{kg} / \mathrm{day})$ and clozapine (60 $\mathrm{mg} / \mathrm{kg} /$ day) in their drinking water. Similar to our previous findings, clozapine affected EAE disease course (Figure 7.2A) and weight (Figure 7.2B) as previously demonstrated. Clozapine delayed the onset of EAE (Figure 7.2C). Peak disease score was not significantly different between experimental groups in these experiments (Figure 7.2D). Clozapine reduced disease burden of EAE when given alone or in combination with L-DOPA (Figure 7.2E). L-DOPA alone did not affect disease onset (Figure 7.2C) nor did it affect overall disease burden (Figure 7.2E). When L-DOPA was given together with clozapine it did not significantly affect the protection provided by clozapine (Figure 7.2E), indicating that L-DOPA does not improve the therapeutic efficacy of $60 \mathrm{mg} / \mathrm{kg} /$ day clozapine. 

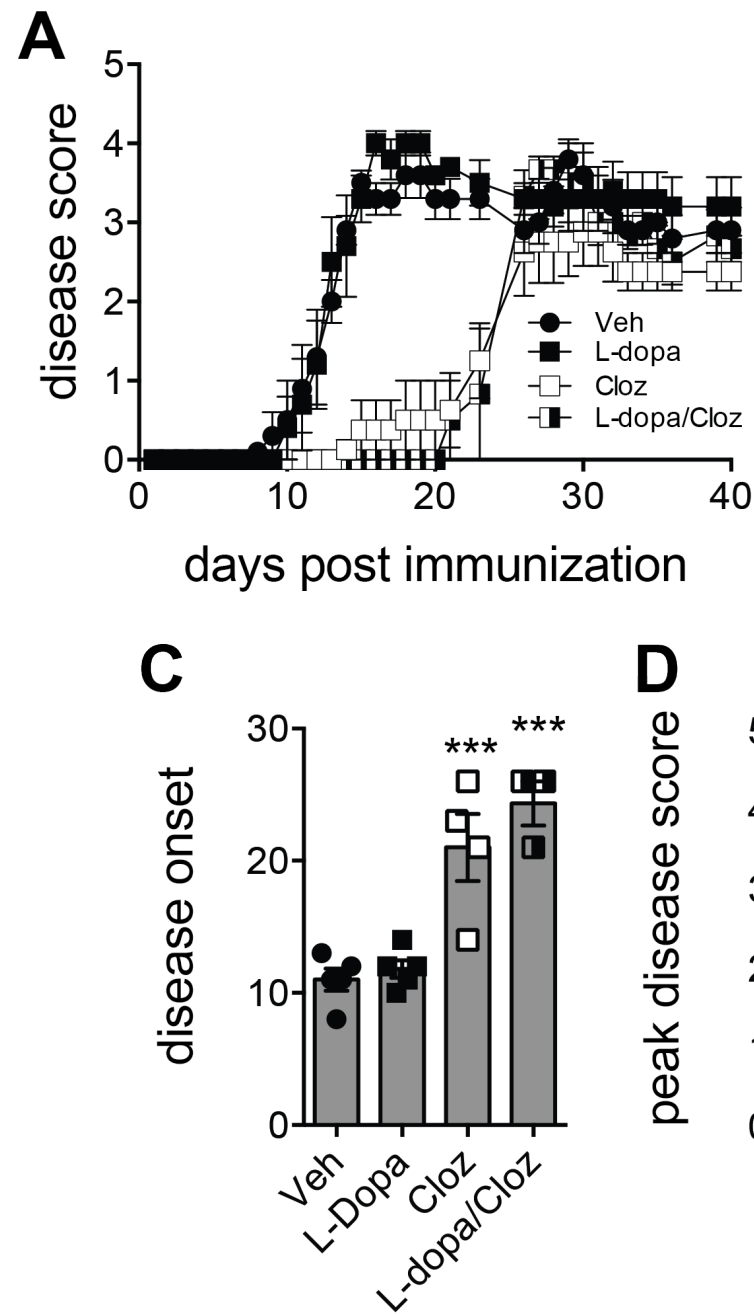

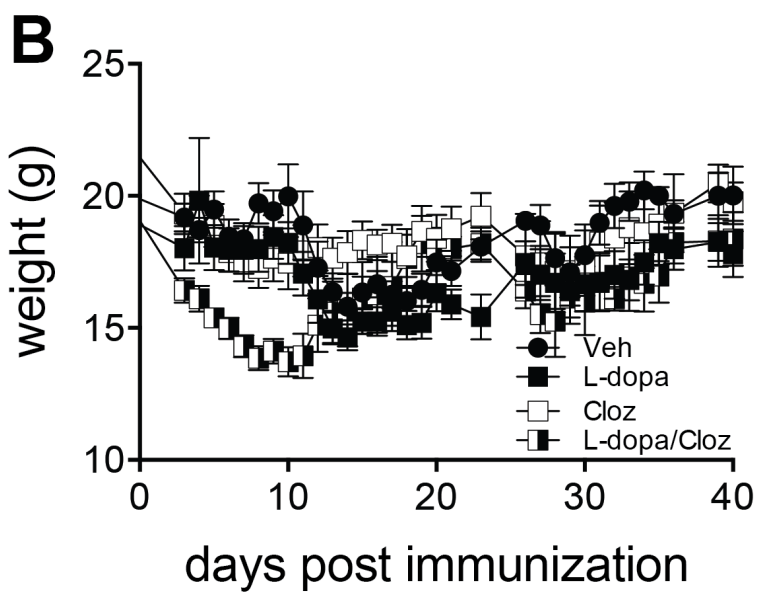

E

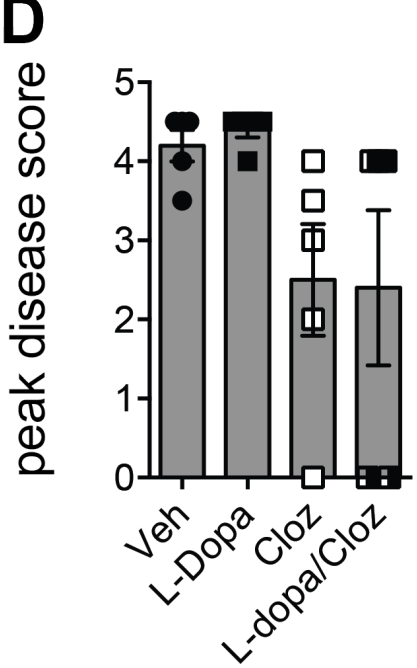

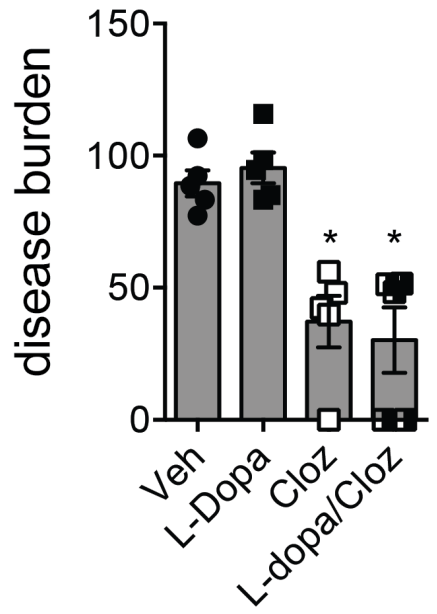

\title{
Figure 7.2 L-DOPA does not improve efficacy of clozapine during EAE
}

\begin{abstract}
A-E C57BL/6J mice were treated with L-DOPA $(100 \mathrm{mg} / \mathrm{kg} /$ day $)$, clozapine $(60 \mathrm{mg} / \mathrm{kg} / \mathrm{day})$ or both in their drinking water one day prior to EAE induction. A Mice were scored daily for disease ( 0 ; normal - 5; moribund) and $\mathbf{B}$ weighed to monitor overall health. C Disease onset of EAE mice only (d.p.i) D Maximum disease score reached (d.p.i). E Disease burden (AUC). Shown are means and SEM of individual mice from one experiment $(\mathrm{n}=5$ mice in each group). Statistical analyses by one-way ANOVA and Dunnett's multiple comparison test. $* \mathrm{p}<0.05, * * * \mathrm{p}<0.001$.
\end{abstract}

Since clozapine is very effective at suppressing EAE disease at the dose used in this study, the experiment was repeated using a suboptimal dose of $10 \mathrm{mg} / \mathrm{kg} /$ day of clozapine with the intention of investigating whether L-DOPA was able to synergize with a suboptimal dose of clozapine. As expected, when clozapine was given at this suboptimal dose, no significant improvement in disease course (Figure 7.3A) or weight (Figure 7.3B) was observed. Clozapine at the lower dose did not improve disease onset (Figure 7.3C), peak disease score 
(Figure 7.3D) or overall disease burden (Figure 7.3E). Likewise, L-DOPA alone did not significantly affect the disease course as previously observed (Figure 7.3A) and although the combination of L-DOPA and clozapine did not have any effect on disease course, it did delay the onset of disease significantly albeit modestly (Figure 7.3C). Taken together, these experiments indicate that L-DOPA does not substantially enhance the protective effect of clozapine.

A

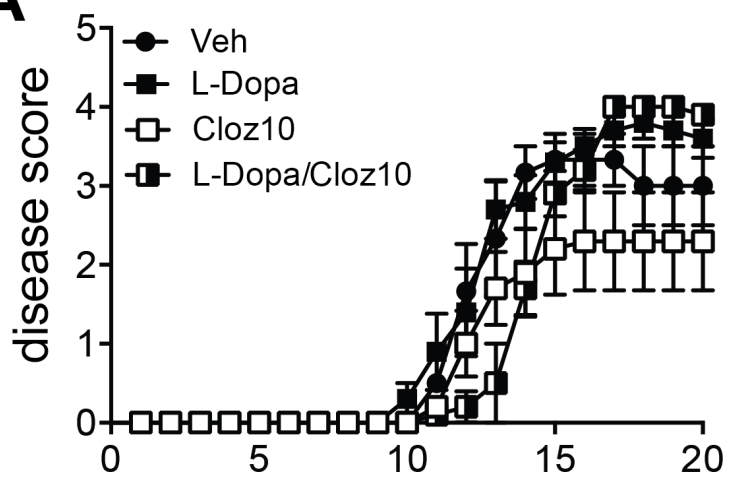

days post immunization
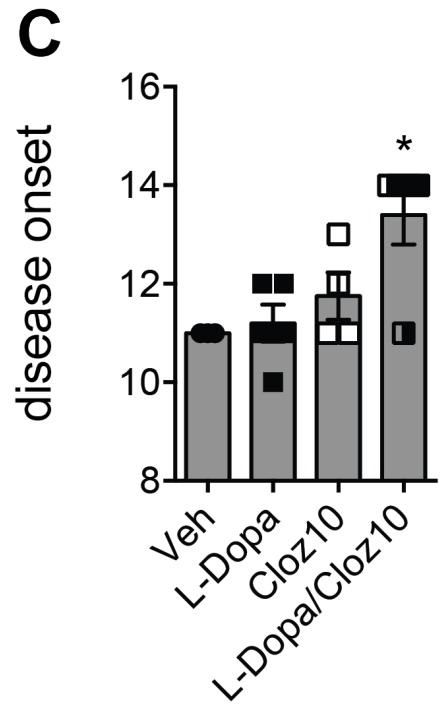

B

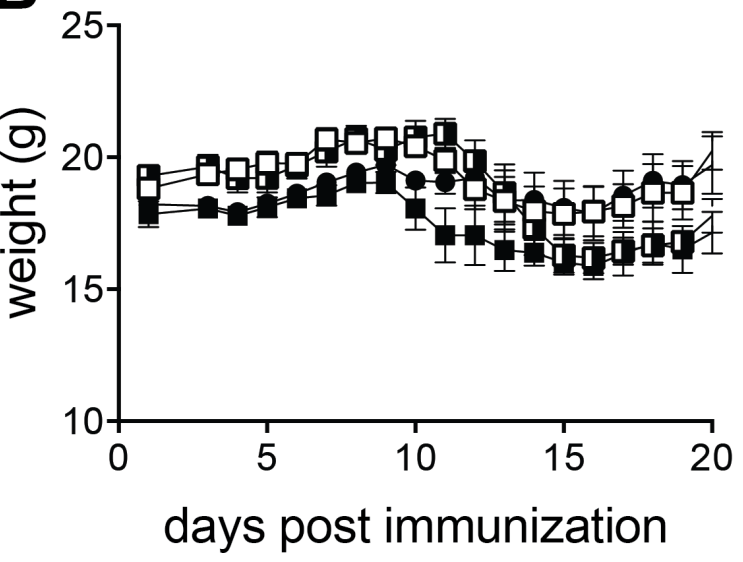

E

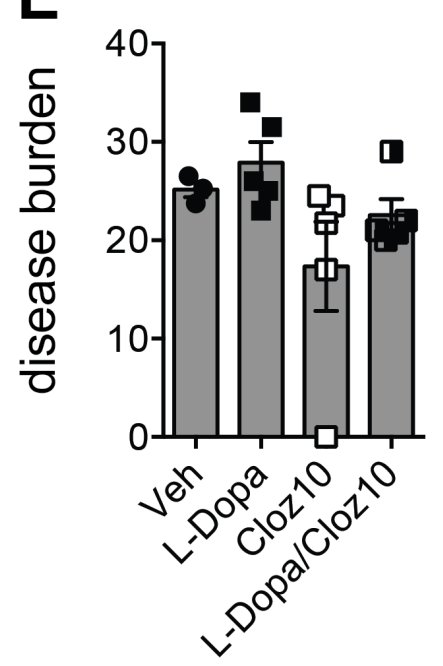

Figure 7.3 L-DOPA does not improve efficacy of low dose clozapine during EAE

A-E C57BL/6J mice were treated with L-DOPA (100 mg/kg/day), clozapine $(10 \mathrm{mg} / \mathrm{kg} / \mathrm{day})$ or both in their drinking water one day prior to EAE induction. A Mice were scored daily for disease ( 0 ; normal - 5; moribund) and $\mathbf{B}$ weighed to monitor overall health. C Disease onset of EAE mice only (d.p.i) D Maximum disease score reached (d.p.i). E Disease burden (AUC). Shown are means and SEM of individual mice from one experiment $(\mathrm{n}=5$ mice in each group). Statistical analyses by one-way ANOVA and Dunnett's multiple comparison test. $* \mathrm{p}<0.05$. 


\subsubsection{Risperidone and fluoxetine do not synergize to enhance protection from EAE}

Considering that GSK-3 modulation is effective for treating EAE and that both risperidone and fluoxetine have previously been reported in the literature to have synergistic effects on the inhibition of GSK-3, it was worthwhile investigating whether this combinational treatment would be more effective at reducing disease during EAE. In contrast to previous work from our group, when mice were given risperidone at $3 \mathrm{mg} / \mathrm{kg} /$ day, only a modest effect on EAE disease and weight was observed that did not reach statistical significance (Figure 7.4A and B). This equated to a similar disease burden experienced by all experimental groups (Figure 7.4C). No difference in disease onset was observed (Figure 7.4D). These results indicate that administering fluoxetine and risperidone together does not improve the therapeutic efficacy of either agent alone. 


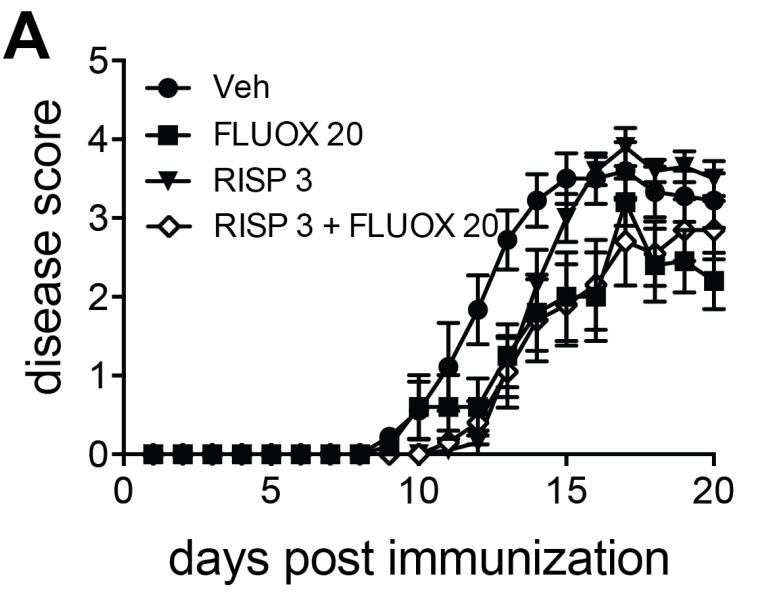

C

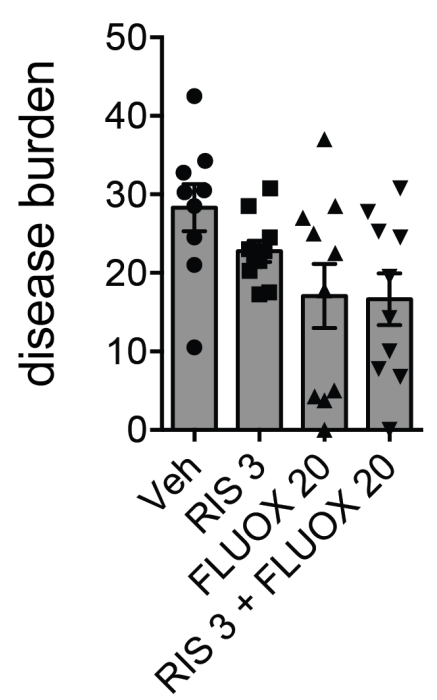

B

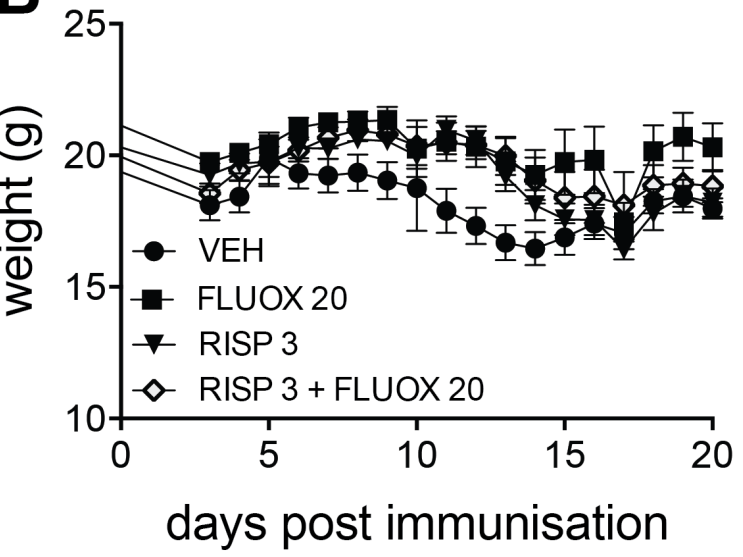

E

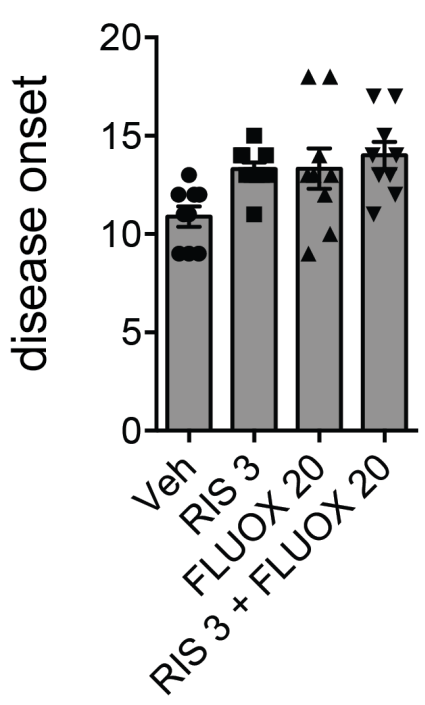

\section{Figure 7.4 Co-administration of risperidone and fluoxetine does not improve EAE}

disease

A-E C57BL/6J mice were treated with fluoxetine (FLUOX 20; Fluoxetine $20 \mathrm{mg} / \mathrm{kg} / \mathrm{day}$ ) and/or risperidone (RISP 3; Risperidone $3 \mathrm{mg} / \mathrm{kg}$ /day) in drinking water available ad libitum one day prior to EAE induction. A Mice were scored daily for disease ( 0 ; normal -5 ; moribund) and $\mathbf{B}$ weighed to monitor overall health. $\mathbf{C}$ Disease burden of mice as assessed by AUC analysis. D Peak disease score of mice. E Disease onset of mice that got sick. Shown are means and SEM of individual mice from two independent experiments combined. $\mathrm{n}=$ 10 mice in each experimental group. Statistical analyses by one-way ANOVA and Dunnett's multiple comparison test.

To further examine whether risperidone and fluoxetine affected the immune response during EAE as originally hypothesized, splenocytes were isolated 20 d.p.i and re-stimulated in vitro with $\mathrm{MOG}_{35-55}$ peptide. Mice treated with risperidone, fluoxetine or both produced similar levels of IFN- $\gamma$ to mice that received vehicle alone (Figure 7.5A). Similarly, no difference in IL-17A responses were found in mice that received risperidone, fluoxetine or both when 
compared to vehicle controls (Figure 7.5B), showing that treatment does not alter antigenspecific recall responses during EAE.

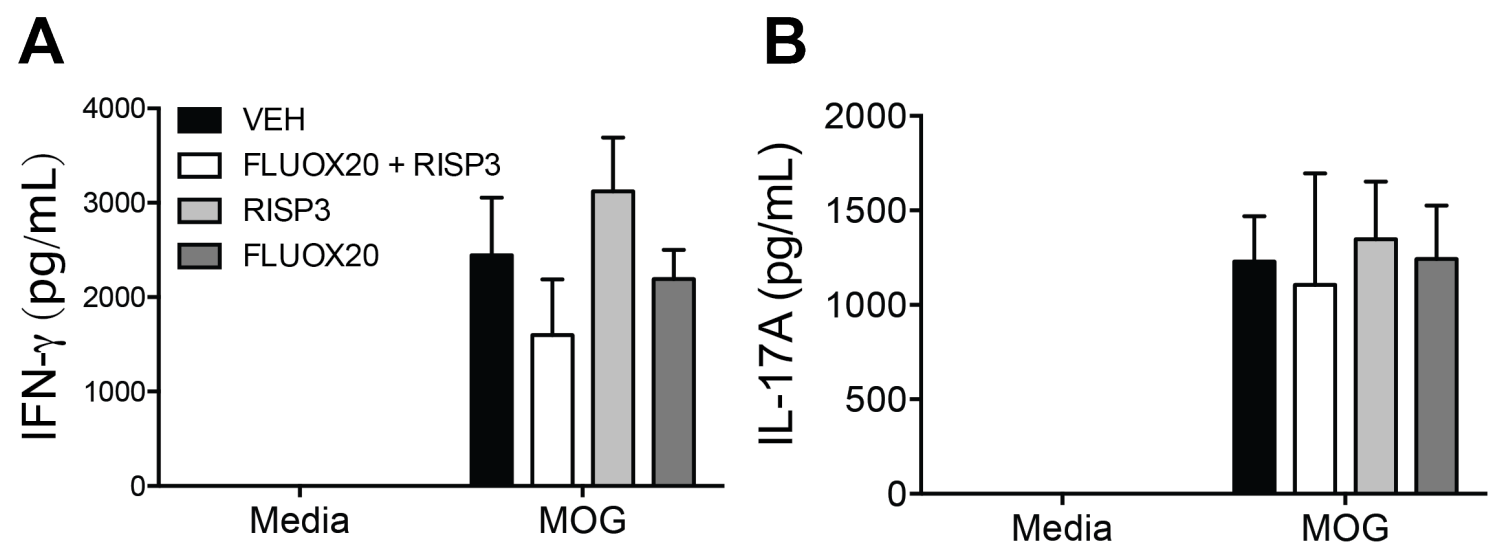

Figure 7.5 Risperidone and Fluoxetine does not alter MOG-specific recall response

A-B Splenocytes were isolated 20 d.p.i, cultured and re-stimulated with $\mathrm{MOG}_{35-55}$ peptide $(50 \mu \mathrm{g} / \mathrm{mL})$ for 72 hours. A IFN- $\gamma$ and B IL-17A in the supernatant were measured by ELISA. Shown are means and SEM of individual mice conducted in triplicate representative of two independent experiments. $n=5$ mice in each experimental group. Statistical analyses by one-way ANOVA and Dunnett's multiple comparison test. 


\subsection{Discussion}

The aim of the experiments in this chapter was to investigate the use of other therapeutic agents approved for other psychiatric diseases and to explore their mechanism for treating EAE as a potential novel therapy for MS in humans. Specifically, this chapter presented pilot studies investigating the effect of treating mice with Levodopa/Carbidopa in combination with clozapine and also using a combination of risperidone/fluoxetine on the disease course of EAE. Under the experimental conditions described, no improvement in EAE disease was observed over clozapine alone at sub-optimal or optimal clozapine doses when various doses of L-DOPA were given to mice. Furthermore, risperidone and fluoxetine co-administration was not significantly more effective at modifying EAE disease, indicating that although fluoxetine has potential as a therapeutic, risperidone does not improve efficacy and this combination may not be an effective candidate for future MS therapeutics.

Dopamine is a neurotransmitter that is known for its immune modulating properties and previously published research as well as work presented in this chapter shows that dopamine can directly suppress the production of IL-12 and increase IL-10 in macrophages indicating that clozapine can alter their activation. IL-12 is an inflammatory cytokine and in the context of EAE, and its p40 subunit is essential for the induction of Th1 and Th17 cells that mediate inflammation in the CNS. IL-12p40/- mice fail to develop Th1 and Th17 cells and are consequently resistant to EAE (Cua et al., 2003). In contrast, IL-10 is a regulatory cytokine that negatively regulates the differentiation and expansion of effector $\mathrm{T}$ cells and are critical for maintaining self-tolerance (O'Garra, Barrat, Castro, Vicari, \& Hawrylowicz, 2008).

In the clinic, L-DOPA is commonly used to treat the symptoms of Parkinson's disease that arise from the loss of dopamine producing neurons (Lotharius \& Brundin, 2002). The most effective treatments for Parkinson's disease is L-DOPA, which is a dopamine precursor and is used to increase extracellular dopamine (Abercrombie, Bonatz, \& Zigmond, 1990) in the CNS as it is able to be transported across the BBB (Kageyama et al., 2000). Little research has been conducted investigating the effect of L-DOPA on the immune response in intact organisms, but a few studies have been conducted using dopamine in in vitro culture of macrophages and $\mathrm{T}$ cells, including ours, which have shown that dopamine can directly alter LPS stimulated macrophages towards a less inflammatory state. In this study, animals were orally administered Sinemet ${ }^{\circledR}$ as a method of increasing extracellular dopamine in the CNS to investigate whether additional dopamine in the CNS can modify EAE disease. 
Given that inflammatory lesions in EAE are composed mainly of macrophages derived from blood monocytes that are essential for EAE disease, we questioned whether exogenous dopamine present in the CNS could reduce EAE disease by altering the activation of macrophages similar to what we observe in in vitro cultured macrophages stimulated with LPS. To achieve this, Sinemet ${ }^{\circledR}$ was used because this formula contains a combination of L-DOPA and Carbidopa. The dopamine precursor L-DOPA can cross the BBB and is used to increase CNS dopamine as dopamine is not able to cross the BBB. L-DOPA is converted to dopamine by DOPA decarboxylase and therefore requires the use of the peripherally restricted DOPA decarboxylase inhibitor, Carbidopa, be used to limit dopamine conversion to the CNS and not the periphery. Limiting L-DOPA conversion to the CNS is essential as peripheral activation of dopaminergic receptors can cause a range of undesirable side effects like nausea and vomiting. Mice were given doses of 50, 100 and $150 \mathrm{mg} / \mathrm{kg} /$ day of L-DOPA/Carbidopa in their drinking water one day prior to EAE induction. These doses were expected to be of appropriate range to experiment as $100 \mathrm{mg} / \mathrm{kg} /$ day were previously shown to alter $\mathrm{T}$ cell immune responses in BALB/c mice (Boukhris, Kouassi, Descotes, Cordier, \& Revillard, 1987).

Because it was observed that clozapine and dopamine had an additive effect on the suppression of IL-12 production by macrophages; mice were then co-treated with L-DOPA/Carbidopa and clozapine to determine whether a greater protection from EAE could be observed. While clozapine was protective, the addition of L-DOPA/Carbidopa did not affect disease nor did it improve the efficacy of high and low doses of clozapine. Contrary to initial hypotheses, these results indicate that treating mice with clozapine and L-DOPA/Carbidopa may not be a better therapeutic strategy than clozapine alone.

These conclusions are limited by a few possible factors. As described in the methods section, the Sinemet ${ }^{\circledR}$ formula was used in tablet form and dissolved in water as an approach of safely adding L-DOPA/Carbidopa into drinking water. While great care was taken to maximize solubility, it is not certain whether the correct dose of Sinemet® was in fact dissolved in the water as the solution contains contaminants from the tablet formulation that are insoluble in water, making the visual observation of dissolving material in the solution impossible. The CNS levels of dopamine or L-DOPA in the CNS were not measured and it therefore cannot be 
concluded that L-DOPA/Carbidopa treatment significantly increased CNS levels of dopamine in these experimental mice. Finally, these studies were limited to the use of a small range of doses and may be improved by addition of higher doses of L-DOPA/Carbidopa. It may then be worthwhile conducting more experiments using pure L-DOPA and Carbidopa to ensure that the correct dosage is given to mice.

Dopaminergic receptors typically signal through cAMP dependent and independent mechanisms and both of these have potential to alter the immune response. cAMP independent mechanisms includes the $\beta$-arrestin pathway that activates the Akt-GSK3 pathway independent of G proteins (J.-M. Beaulieu, Del' Guidice, Sotnikova, Lemasson, \& Gainetdinov, 2011) and was demonstrated in vivo when chronic L-DOPA treatment activated Akt and inhibited GSK3 in the striatum of animals depleted of dopamine (Bychkov, Ahmed, Dalby, \& Gurevich, 2007). Given that GSK3 inhibitors can directly suppress EAE disease and that both dopamine and clozapine have been reported to inhibit GSK3, it was proposed that co-administering LDOPA could have potential to improve therapeutic response to clozapine alone, however, since no improvement was observed, it is possible that L-DOPA and clozapine does not inhibit GSK3 sufficiently to reduce EAE disease. Furthermore, these experiments used the Sinemet formulation of L-DOPA which includes carbidopa to limit peripheral metabolism of L-DOPA to dopamine, therefore these experiments limit the effects of L-DOPA to the CNS whereas previously published work investigating the use of GSK3 inhibitors in EAE were administering compounds peripherally and are not limited to the CNS but also active in the periphery.

There is already considerable evidence for glycogen synthase kinase- $3 \beta$ and its role in regulating immune responses and these effects may underlie the mechanism by which psychiatric agents are therapeutic during EAE. EAE is a disease that requires the activity of Th1 and Th17 cells and one hypothesis is that agents that inhibit glycogen synthase kinase-3 $\beta$ suppress the differentiation of $\mathrm{T}$ lymphocytes to gain pathogenic function due to the dependence of Th1 and Th17 cells on glycogen synthase kinase-3 $\beta$. This is demonstrated clearly in a study where inhibition of GSK-3 specifically prevents differentiation to Th1 and Th17 cells but not towards Treg (Beurel et al., 2013). Furthermore, specifically inhibiting GSK3 $\beta$ during EAE is by itself sufficient to reduce disease (Beurel et al., 2013; E. Beurel et al., 2011). GSK3 $\beta$ is also involved in regulation of activated macrophages/monocytes. The previous chapter verified reports that the Akt-GSK3 pathway is a key regulator of LPS induced cytokine production by macrophages by using specific inhibitors of Akt and GSK3 where 
inhibiting GSK3 activity suppresses IL-12 and increases IL-10 secretion in primary macrophages, an effect that parallels what is observed with risperidone and clozapine. These studies indicate that GSK3 promotes inflammatory responses in both $\mathrm{T}$ cells and macrophages/monocytes and while the precise mechanism of action of these agents remains to be established, there is already compelling evidence to indicate that modifying GSK3 $\beta$ activity may be a promising target for the treatment of EAE and MS.

The GSK-3 $\beta$ is a pathway that is modulated not only by atypical antipsychotic agents but is also a pathway that is shared with multiple other therapeutic agents for psychiatric illness such as fluoxetine and lithium chloride used for treating depression and bipolar disorder respectively. Not only do these agents share the ability to modulate GSK-3 $\beta$; clozapine, risperidone, fluoxetine and lithium are all known to have immune modifying effects and are effective at reducing disease during EAE (De Sarno et al., 2008; D. O'Sullivan et al., 2014; Sacre et al., 2010; Yuan et al., 2012). Moreover, a study by Li et al. in 2007 elegantly showed that when risperidone and fluoxetine are co-administered to mice by i.p injection, phosphorylation of GSK-3 $\beta$ in the cortex, hippocampus and striatum improved over either risperidone or fluoxetine alone (Li et al., 2007), and this combination has proven more effective at shifting stress activated inflammatory macrophages towards the M2 regulatory phenotype (Roman et al., 2012). However, in the experiments performed in this chapter, co-administering risperidone and fluoxetine were not more effective at reducing EAE disease. It is important to note that an oral administration route of delivery was utilized in our experiments which may have altered efficacy, but given that these are orally administered agents in humans, it was a more clinically relevant finding for the treatment of human diseases.

\subsection{Conclusions}

Experimental results from this chapter show that L-DOPA/Carbidopa does not improve the efficacy of sub-optimal and optimal doses of clozapine during EAE when orally administered chronically. Likewise, the risperidone and fluoxetine combination which had potential to greatly alter GSK3-B phosphorylation in the brain of mice were not effective at reducing disease during chronic oral administration in EAE. These results indicate that LDOPA/Clozapine and risperidone/fluoxetine are unlikely candidates for therapeutics during MS. 
Chapter 8: General Discussion 


\subsection{Summary}

This study focused on investigating the immunological and molecular mechanisms by which the atypical antipsychotic clozapine modulates the immune response to reduce disease in EAE. This study demonstrated that while clozapine effectively delayed the onset and limited progression to severe disease, protection from disease was not associated with defective antigen-specific $\mathrm{T}$ cell responses during the induction or effector phase of disease. In vitro experiments indicated that clozapine had potential to impair pathogenic Th1/Th17 responses although these effects were not observed in vivo. In addition, in vitro experiments indicated that clozapine had the potential to promote the development and function of Treg although these were proven dispensable for EAE protection by clozapine, indicating that clozapine treatment protects from EAE by an alternative mechanism. While no defect in T cell responses was measured during EAE, analysis of microglia and monocytes in the CNS demonstrated that clozapine can suppress their activation, and this alteration in activation was shown to be a direct effect by using in vitro cultured macrophages. Clozapine-treated mice had remarkably less infiltration of inflammatory cells into the CNS raising the possibility that reduced microglia and monocyte activation minimized recruitment to the CNS. However, clozapine was not effective at preventing disease when EAE was induced by adoptive transfer of encephalitogenic $\mathrm{T}$ cells indicating that a multi-faceted mechanism is likely to be involved. Overall, these results show that clozapine can suppress the activation of microglia and macrophages in the CNS, and this effect may play a significant role in disease protection from EAE.

\subsection{The potential mechanism of action of clozapine during EAE}

This study aimed to investigate the mechanism by which clozapine was able to reduce disease during EAE. There are currently no studies that have investigated the effects of clozapine on the immune response in vivo, however, a few have recognized its immunomodulatory properties. Studies have shown that immune cells such as macrophages and $\mathrm{T}$ cells are regulated by many neurotransmitters, including dopamine receptors (McKenna et al., 2002; R. Pacheco, Prado, Barrientos, \& Bernales, 2009; R. Pacheco et al., 2010) and signalling by these could be targeted by clozapine (Brunello, Masotto, Steardo, Markstein, \& Racagni, 1995), making the elucidation of the precise mechanism a complex task. This task was tackled using a systematic approach that investigated important aspects of EAE pathogenesis as potential targets of clozapine. This approach indicated that clozapine is unlikely to have affected antigen- 
specific $\mathrm{T}$ cell responses in the periphery as a way of reducing disease and suggests an alternative mechanism. Chronic activation and infiltration of microglia and monocytes are a hallmark of MS and EAE and are major contributors to neurological damage. Chronic microglial activation-induced neurodegeneration has been observed in many different diseases including Alzheimer's disease (Heneka, Kummer, \& Latz, 2014) and schizophrenia (Müller et al., 2015) further supporting the pathological role of microglia in MS. Targeting microglial activation has proven an effective means of reducing EAE disease (J. Zhou et al., 2015) and similarly, limiting the recruitment of CCR $2^{+}$monocytes into the CNS also prevents disease (Ajami et al., 2011). Interestingly, the antibiotic minocycline, known for its capacity to inhibit microglia (Kobayashi et al., 2013), has been shown to have potential in treating MS (Luccarini et al., 2008) and schizophrenia (Miyaoka et al., 2008).

The fact that clozapine effectively inhibits the activation of macrophages and microglia in the CNS has significant implications for the potential mechanism by which it may reduce EAE. During the induction of EAE, microglia in the CNS become activated before the onset of disease or infiltration of peripheral immune cells (E. D. Ponomarev, Shriver, Maresz, \& Dittel, 2005) potentially by GM-CSF produced by self-reactive T cells in the periphery, both of which are required for EAE (E. D. Ponomarev et al., 2007). Indeed, selectively preventing the activation of microglia inhibits the induction of EAE (Heppner et al., 2005). In addition, EAE requires that $\mathrm{MHC}$-expressing cells like microglia re-activate self-reactive T cells in the CNS (Tompkins et al., 2002), further highlighting the importance of microglia activation for EAE induction and progression. Further experimental investigation is required to conclude with certainty that clozapine limits disease by inhibiting microglia, although it is postulated that clozapine can also affect other immune parameters in combination.

While this study focused mainly on the functional activity of T cells and myeloid cells, the potential that clozapine alters other extraneous physiological aspects cannot be excluded. For example, this study did not investigate the effects of clozapine on the trafficking of immune cells across the $\mathrm{BBB}$, however, it can be inferred that clozapine is unlikely to affect access to the BBB as it was carefully demonstrated in this study that adoptive transfer of encephalitogenic T cells into clozapine treated animals did not prevent the induction of EAE.

Nevertheless, investigation into the effect of clozapine on the trafficking of immune cells across the BBB endothelium would be highly beneficial in providing insight into the mechanism of action of clozapine during EAE. In the steady state, brain endothelial cells 
express low levels of cell adhesion molecules and can be up-regulated during inflammation. Similarly, activated immune cells including effector $\mathrm{T}$ cells express cell adhesion molecules such as the $\alpha 4 \beta 1$-integrin that is the target of MS therapeutic Natalizumab; allowing them to traffic into the CNS (Ted A. Yednock et al., 1992). Interactions between leukocytes and brain endothelial cells through cell adhesion molecules are dependent on GPCR signalling (Adamson et al., 2002; Chigaev et al., 2008; Shamri et al., 2002). Currently, there are no studies that have investigated how clozapine affects the expression of cell adhesion molecules on immune or endothelial cells. In addition, since the identified targets of clozapine are primarily GPCR (Catapano \& Manji, 2007), there is potential that signalling pathways through cell adhesion molecules are perturbed by signalling alterations induced with clozapine treatment; potentially causing poorer trafficking into the CNS. Investigation into these possibilities would be useful in determining the precise mechanism of action of clozapine during EAE.

Although there is not enough evidence to indicate whether clozapine is able to alter the trafficking of immune cells into the CNS; this study provides some evidence to suggest that clozapine does not have a significant effect on CNS trafficking based on the adoptive transfer of EAE approach. This model is commonly used to study the effector phases of disease pathogenesis as the induction phase of disease can be isolated from the analysis. For example, in the adoptive transfer of EAE approach, T cells that are myelin-specific and activated are transferred into recipients such that these $\mathrm{T}$ cells do not need to undergo priming and antigenpresentation by host dendritic cells. Secondly, since no immunisation with myelin antigens were given to the recipients, there is no pool of antigen from which more host naïve $\mathrm{T}$ cells could be activated, thus, it is considered a useful model for studying the effector phases of disease (C. Kim \& Tse, 1993; McCarthy, Richards, \& Miller, 2012). If clozapine were to directly have an effect on the endothelial cells of the BBB one could expect that the clozapine treated mice would experience less disease than untreated mice. In contrast, this study showed that clozapine treatment did not have an effect on disease when EAE was induced by adoptive transfer.

This was an interesting finding since clozapine was highly effective at reducing disease when EAE was induced actively by immunisation. This could indicate that clozapine significantly affects the induction phase of EAE, but has less or no effect on the effector phases of disease. However, this possibility is confounded by the findings presented in this thesis showing that clozapine treatment does not alter the development of antigen specific CD4 T cells. One other possibility is that this result is an artefact of the artificial model of disease and it could be 
expected that a greater difference might be observed if the culture conditions or the number of antigen specific $\mathrm{T}$ cells adoptively transferred were optimised further. This would address the possibility that the adoptive transfer of EAE protocol generates $T$ cells that are excessively encephalitogenic and cannot be controlled by clozapine treatment. In support of this possibility, a similar adoptive transfer EAE model has previously been shown to induce increased expression of the microglial/macrophage activation marker Iba-1, isolectin and GFAP in astrocytes (Mannara et al., 2012).

This study is also limited by the absence of any investigation as to the effect of clozapine on astrocytes, given their activation is also crucial for EAE induction (Brambilla et al., 2014); this aspect merits further investigation.

\subsection{Clinical Implications}

MS is a highly heterogeneous disease and is broadly characterized into three major forms. Most patients when first diagnosed have a relapsing-remitting course of disease. Each relapse is associated with CNS inflammation induced by infiltration of inflammatory cells (Frischer et al., 2009). This feature of RRMS is targeted by all of the currently approved treatment options, which target cells in the periphery; however, when disease enters the progressive stages of disease, neurodegeneration is uncoupled from the peripheral immune system. BBB disruptions and leukocyte infiltration are no longer apparent in the progressive stages; consequently, drugs that were previously used to treat RRMS are no longer effective, leaving patients to progress continually, losing up to $84 \%$ of axonal volume (Bjartmar, Kidd, Mork, Rudick, \& Trapp, 2000). Studies indicate that much of the neurodegeneration that occurs in progressive forms of MS can be attributed to chronic microglial activation (Felts et al., 2005; Ferguson, Matyszak, Esiri, \& Perry, 1997; Prineas et al., 2001) and may be treated with drugs that inhibit microglial activation.

Alternative therapies have been proposed for progressive MS patients and one of the most intriguing possibilities is to target the remyelination process. Remyelination is observed in both MS and EAE lesions (Merkler, Ernsting, Kerschensteiner, Bruck, \& Stadelmann, 2006; Patani, Balaratnam, Vora, \& Reynolds, 2007). Unfortunately, as disease progresses with time and age, the rate of remyelination diminishes (Shields, Gilson, Blakemore, \& Franklin, 1999) which likely results in the failure of repair processes to combat chronic demyelination. This is probably part of the reason why immune modulating agents can reduce relapses in MS without 
preventing disease progression. Prospective therapeutic agents must therefore achieve three general functions; cross the BBB to act on the CNS directly, suppress the demyelination process by inhibiting inflammation and promote the repair or remyelination of damaged neurons. Recent studies suggest that clozapine has potential to stimulate re-myelination in oligodendrocytes (Steiner et al., 2014). Furthermore, it has been shown in our laboratory (unpublished data) by Nikki Templeton that clozapine can enhance neurological deficits induced by cuprizone-induced demyelination, suggesting that clozapine may promote remyelination. In addition, clozapine is demonstrated to protect neurons from inflammatory damage (X. Hu et al., 2012). These studies in conjunction with this study indicate that clozapine may help promote CNS repair and inflammatory damage. Moreover, orally-administered compounds that can cross the blood brain barrier such as clozapine may offer better access to the central nervous system than currently available protein or antibody-based treatments (di Nuzzo, Orlando, Nasca, \& Nicoletti, 2014). The data presented in this thesis supports the possibility that clozapine is neuroprotective in ways that are also independent of the immune response. For example, the reduced expression of activation markers on microglia and macrophages are not significantly different in clozapine treated mice compared to vehicle treated mice 20 days post immunisation, yet the improvement in disease score is maintained. Further experimental studies will be required to determine whether clozapine is neuroprotective in an immune independent manner in the EAE model.

Altogether, the anti-inflammatory, neuroprotective and myelin-promoting properties of clozapine meets all of the fundamental requirements of a potential therapeutic for the progressive stages of MS and further investigation in clinical efficacy is warranted.

\subsection{Future Directions}

This study has shown that clozapine inhibits the activation of microglia and monocytes in the CNS. With the current data, it is hypothesized that clozapine inhibits early activation of microglia in the CNS, limiting the entry of inflammatory cells into the CNS. Although this study showed that clozapine does indeed alter microglia activation, the experimental data currently does not definitively show that clozapine prevents the activation of microglia during the induction of EAE, which subsequently prevents peripheral infiltration. To address this question, it must first be shown that microglia are indeed activated after EAE induction prior to the infiltration of peripherally-derived immune cells. It must also be shown that clozapine inhibits the production of chemoattractant and inflammatory mediators by microglia early 
during the induction phase. To achieve this, microglia can be cell sorted by fluorescence assisted cell sorting early during the induction phase where no peripheral infiltration of other immune cells can be detected. To distinguish resident microglia, the P2Y12 receptor can be used as it was recently identified as a unique marker of resident microglia (Butovsky et al., 2014). Microglia can then be isolated and analysed for mRNA expression of CCL2, CCL3, CCL4, CCL5, IP-10 and Fractalkine to compare microglial expression of these between vehicle and clozapine-treated mice. If clozapine does indeed inhibit the expression of chemoattractants and inflammatory mediators before the infiltration of other immune cells, then it could be a mechanism by which clozapine limits the infiltration of immune cells to the CNS and subsequently reduces EAE.

Furthermore, while this study was unable to confirm previous reports of clozapine having inhibitory activity on the constitutively active enzyme GSK-3 $\beta$, it is still a possible molecular mechanism by which clozapine reduces EAE as inhibition of this enzyme is consistent with our observations that show clozapine is able to alter T cell differentiation and modify LPS induced cytokine production. To demonstrate this notion in vivo during EAE, GSK$3 \beta^{\mathrm{S} 21 \mathrm{~A} / \mathrm{S} 21 \mathrm{~A} / \mathrm{S} 9 \mathrm{~A} / \mathrm{S} 9 \mathrm{~A}}$ knock-in mice can be used which express a GSK-3 $\beta$ that cannot be inhibited by phosphorylation and consequently develop more severe EAE disease (E. Beurel, W.-I. Yeh, S. M. Michalek, L. E. Harrington, \& R. S. Jope, 2011). GSK-3 $\beta$ from these mice cannot be inhibited by clozapine treatment, and it is hypothesized that clozapine would not be effective in these mice. Moreover, bone marrow chimeric mice using GSK-3 $\beta^{\mathrm{S} 21 \mathrm{~A} / \mathrm{S} 21 \mathrm{~A} / \mathrm{S} 9 \mathrm{~A} / \mathrm{S} 9 \mathrm{~A}} \mathrm{knock}-\mathrm{in}$ and WT mice can be generated to determine the precise immune cell types involved in EAE protection by clozapine.

Finally, this study did not investigate other potential signalling changes that may occur with clozapine treatment. One study shows that olanzapine may activate the mTOR pathway (Schmidt et al., 2013). Since clozapine is a structurally similar compound, it would be worth investigating this pathway as there have been no studies investigating the effect of clozapine on the mTOR pathway. Another study has indicated that atypical antipsychotic agents including clozapine may also activate the TGF- $\beta$ pathway (Cohen et al., 2013) and this pathway has significant merit as another potential mechanism by which clozapine is able to reduce EAE given the known requirement for TGF- $\beta$ signalling in Treg differentiation. 


\section{References}


Abbott, N. J., Ronnback, L., \& Hansson, E. (2006). Astrocyte-endothelial interactions at the blood-brain barrier. Nat Rev Neurosci, 7(1), 41-53. doi:10.1038/nrn1824

Abercrombie, E. D., Bonatz, A. E., \& Zigmond, M. J. (1990). Effects ofl-DOPA on extracellular dopamine in striatum of normal and 6-hydroxydopamine-treated rats. Brain Res, 525(1), 36-44. doi:http://dx.doi.org/10.1016/0006-8993(90)91318-B

Acuto, O., \& Michel, F. (2003). CD28-mediated co-stimulation: a quantitative support for TCR signalling. Nat Rev Immunol, 3(12), 939-951.

Adamson, P., Wilbourn, B., Etienne-Manneville, S., Calder, V., Beraud, E., Milligan, G., .. . Greenwood, J. (2002). Lymphocyte trafficking through the blood-brain barrier is dependent on endothelial cell heterotrimeric G-protein signaling. Faseb j, 16(10), 1185-1194. doi:10.1096/fj.02-0035com

Ajami, B., Bennett, J. L., Krieger, C., McNagny, K. M., \& Rossi, F. M. (2011). Infiltrating monocytes trigger EAE progression, but do not contribute to the resident microglia pool. Nat Neurosci, 14(9), 1142-1149. doi:10.1038/nn.2887

Akira, S., \& Takeda, K. (2004). Toll-like receptor signalling. Nat Rev Immunol, 4(7), 499511.

Akirav, E. M., Bergman, C. M., Hill, M., \& Ruddle, N. H. (2009). Depletion of CD4(+)CD25(+) T cells Exacerbates Experimental Autoimmune Encephalomyelitis Induced by Mouse, but Not Rat, Antigens. Journal of neuroscience research, 87(15), 3511-3519. doi:10.1002/jnr.21981

Aktas, O., Kury, P., Kieseier, B., \& Hartung, H. P. (2010). Fingolimod is a potential novel therapy for multiple sclerosis. Nat Rev Neurol, 6(7), 373-382. doi:10.1038/nrneurol.2010.76

Al-Amin, M. M., Nasir Uddin, M. M., \& Mahmud Reza, H. (2013). Effects of Antipsychotics on the Inflammatory Response System of Patients with Schizophrenia in Peripheral Blood Mononuclear Cell Cultures. Clinical Psychopharmacology and Neuroscience, 11(3), 144-151. doi:10.9758/cpn.2013.11.3.144

Alegre, M.-L., Frauwirth, K. A., \& Thompson, C. B. (2001). T-cell regulation by CD28 and CTLA-4. Nat Rev Immunol, 1(3), 220-228.

Ascherio, A., \& Munger, K. L. (2007). Environmental risk factors for multiple sclerosis. Part I: the role of infection. Ann Neurol, 61(4), 288-299. doi:10.1002/ana.21117 
Bachmanov, A. A., Reed, D. R., Beauchamp, G. K., \& Tordoff, M. G. (2002). Food Intake, Water Intake, and Drinking Spout Side Preference of 28 Mouse Strains. Behavior genetics, 32(6), 435-443.

Barry, M., \& Bleackley, R. C. (2002). Cytotoxic T lymphocytes: all roads lead to death. Nat Rev Immunol, 2(6), 401-409.

Beaulieu, J.-M., Del' Guidice, T., Sotnikova, T., Lemasson, M., \& Gainetdinov, R. (2011). Beyond cAMP: The Regulation of Akt and GSK3 by Dopamine Receptors. Frontiers in Molecular Neuroscience, 4(38). doi:10.3389/fnmol.2011.00038

Beaulieu, J. M., Gainetdinov, R. R., \& Caron, M. G. (2009). Akt/GSK3 signaling in the action of psychotropic drugs. Annu Rev Pharmacol Toxicol, 49, 327-347. doi:10.1146/annurev.pharmtox.011008.145634

Benson, C., Paylor, J. W., Tenorio, G., Winship, I., Baker, G., \& Kerr, B. J. (2015).

Voluntary wheel running delays disease onset and reduces pain hypersensitivity in early experimental autoimmune encephalomyelitis (EAE). Exp Neurol, 271, 279-290. doi:10.1016/j.expneurol.2015.05.017

Bettelli, E., Pagany, M., Weiner, H. L., Linington, C., Sobel, R. A., \& Kuchroo, V. K. (2003). Myelin Oligodendrocyte Glycoprotein-specific T Cell Receptor Transgenic Mice Develop Spontaneous Autoimmune Optic Neuritis. The Journal of Experimental Medicine, 197(9), 1073-1081. doi:10.1084/jem.20021603

Beurel, E., Kaidanovich-Beilin, O., Yeh, W. I., Song, L., Palomo, V., Michalek, S. M., . . . Jope, R. S. (2013). Regulation of Th1 cells and experimental autoimmune encephalomyelitis by glycogen synthase kinase-3. J Immunol, 190(10), 5000-5011. doi:10.4049/jimmunol.1203057

Beurel, E., Yeh, W.-I., Michalek, S. M., Harrington, L. E., \& Jope, R. S. (2011). Glycogen Synthase Kinase-3 Is an Early Determinant in the Differentiation of Pathogenic Th17 Cells. J Immunol, 186(3), 1391-1398. doi:10.4049/jimmunol.1003511

Beurel, E., Yeh, W. I., Michalek, S. M., Harrington, L. E., \& Jope, R. S. (2011). Glycogen synthase kinase-3 is an early determinant in the differentiation of pathogenic Th17 cells. J Immunol, 186(3), 1391-1398. doi:10.4049/jimmunol.1003511

Bian, Q., Kato, T., Monji, A., Hashioka, S., Mizoguchi, Y., Horikawa, H., \& Kanba, S. (2008). The effect of atypical antipsychotics, perospirone, ziprasidone and quetiapine on microglial activation induced by interferon-gamma. Prog Neuropsychopharmacol Biol Psychiatry, 32(1), 42-48. doi:10.1016/j.pnpbp.2007.06.031 
Bjartmar, C., Kidd, G., Mork, S., Rudick, R., \& Trapp, B. D. (2000). Neurological disability correlates with spinal cord axonal loss and reduced $\mathrm{N}$-acetyl aspartate in chronic multiple sclerosis patients. Ann Neurol, 48(6), 893-901.

Bo, L., Mork, S., Kong, P. A., Nyland, H., Pardo, C. A., \& Trapp, B. D. (1994). Detection of MHC class II-antigens on macrophages and microglia, but not on astrocytes and endothelia in active multiple sclerosis lesions. J Neuroimmunol, 51(2), 135-146.

Bogie, J. F., Stinissen, P., \& Hendriks, J. J. (2014). Macrophage subsets and microglia in multiple sclerosis. Acta Neuropathol, 128(2), 191-213. doi:10.1007/s00401-014$1310-2$

Boukhris, W., Kouassi, E., Descotes, J., Cordier, G., \& Revillard, J. P. (1987). Impaired Tdependent immune response in L-dopa treated BALB/c mice. J Clin Lab Immunol, 23(4), 185-189.

Brambilla, R., Morton, P. D., Ashbaugh, J. J., Karmally, S., Lambertsen, K. L., \& Bethea, J. R. (2014). Astrocytes play a key role in EAE pathophysiology by orchestrating in the CNS the inflammatory response of resident and peripheral immune cells and by suppressing remyelination. Glia, 62(3), 452-467. doi:10.1002/glia.22616

Brosnan, C. F., Bornstein, M. B., \& Bloom, B. R. (1981). The effects of macrophage depletion on the clinical and pathologic expression of experimental allergic encephalomyelitis. J Immunol, 126(2), 614-620.

Bruck, W., Sommermeier, N., Bergmann, M., Zettl, U., Goebel, H. H., Kretzschmar, H. A., \& Lassmann, H. (1996). Macrophages in multiple sclerosis. Immunobiology, 195(45), 588-600. doi:10.1016/s0171-2985(96)80024-6

Brunello, N., Masotto, C., Steardo, L., Markstein, R., \& Racagni, G. (1995). New insights into the biology of schizophrenia through the mechanism of action of clozapine. Neuropsychopharmacology, 13(3), 177-213. doi:10.1016/0893-133x(95)00068-o

Bulek, A. M., Cole, D. K., Skowera, A., Dolton, G., Gras, S., Madura, F., . . Sewell, A. K. (2012). Structural basis for the killing of human beta cells by CD8+ T cells in type 1 diabetes. Nat Immunol, 13(3), 283-289.

doi:http:/www.nature.com/ni/journal/v13/n3/abs/ni.2206.html - supplementaryinformation

Butovsky, O., Jedrychowski, M. P., Moore, C. S., Cialic, R., Lanser, A. J., Gabriely, G., . . . Weiner, H. L. (2014). Identification of a unique TGF-[beta]-dependent molecular and functional signature in microglia. Nat Neurosci, 17(1), 131-143. doi:10.1038/nn.3599 http://www.nature.com/neuro/journal/v17/n1/abs/nn.3599.html - supplementary-information 
Bychkov, E., Ahmed, M. R., Dalby, K. N., \& Gurevich, E. V. (2007). Dopamine depletion and subsequent treatment with 1-DOPA, but not the long-lived dopamine agonist pergolide, enhances activity of the Akt pathway in the rat striatum. Journal of Neurochemistry, 102(3), 699-711. doi:10.1111/j.1471-4159.2007.04586.x

Cadman, E. T., \& Lawrence, R. A. (2010). Granulocytes: effector cells or immunomodulators in the immune response to helminth infection? Parasite Immunol, 32(1), 1-19. doi:10.1111/j.1365-3024.2009.01147.x

Caley, C. F., \& Weber, S. S. (1995). Sulpiride: an antipsychotic with selective dopaminergic antagonist properties. Ann Pharmacother, 29(2), 152-160.

Cannons, J. L., Tangye, S. G., \& Schwartzberg, P. L. (2011). SLAM family receptors and SAP adaptors in immunity. Annu Rev Immunol, 29, 665-705. doi:10.1146/annurevimmunol-030409-101302

Casey, D. E., Haupt, D. W., Newcomer, J. W., Henderson, D. C., Sernyak, M. J., Davidson, M., ... Hennekens, C. H. (2004). Antipsychotic-induced weight gain and metabolic abnormalities: implications for increased mortality in patients with schizophrenia. $J$ Clin Psychiatry, 65 Suppl 7, 4-18; quiz 19-20.

Casrouge, A., Beaudoing, E., Dalle, S., Pannetier, C., Kanellopoulos, J., \& Kourilsky, P. (2000). Size estimate of the alpha beta TCR repertoire of naive mouse splenocytes. $J$ Immunol, 164(11), 5782-5787.

Catapano, L. A., \& Manji, H. K. (2007). G protein-coupled receptors in major psychiatric disorders. Biochimica et Biophysica Acta (BBA) - Biomembranes, 1768(4), 976-993. doi:https://doi.org/10.1016/j.bbamem.2006.09.025

Chang, T. T., Sobel, R. A., Wei, T., Ransohoff, R. M., Kuchroo, V. K., \& Sharpe, A. H. (2003). Recovery from EAE is associated with decreased survival of encephalitogenic T cells in the CNS of B7-1/B7-2-deficient mice. Eur J Immunol, 33(7), 2022-2032. doi:10.1002/eji.200323180

Chen, M.-L., Tsai, T.-C., Lin, Y.-Y., Tsai, Y.-M., Wang, L.-K., Lee, M.-C., \& Tsai, F.-M. (2011). Antipsychotic drugs suppress the AKT/NF-kB pathway and regulate the differentiation of T-cell subsets. Immunology Letters, 140(1-2), 81-91. doi:http://dx.doi.org/10.1016/j.imlet.2011.06.011

Chen, Z., Jalabi, W., Shpargel, K. B., Farabaugh, K. T., Dutta, R., Yin, X., . . Trapp, B. D. (2012). Lipopolysaccharide-Induced Microglial Activation and Neuroprotection against Experimental Brain Injury Is Independent of Hematogenous TLR4. The 
Journal of neuroscience : the official journal of the Society for Neuroscience, 32(34), 11706-11715. doi:10.1523/JNEUROSCI.0730-12.2012

Cherry, J. D., Olschowka, J. A., \& O’Banion, M. K. (2014). Neuroinflammation and M2 microglia: the good, the bad, and the inflamed. J Neuroinflammation, 11(1), 98. doi:10.1186/1742-2094-11-98

Chi, H. (2012). Regulation and function of mTOR signalling in T cell fate decisions. Nat Rev Immunol, 12(5), 325-338.

Chigaev, A., Waller, A., Amit, O., \& Sklar, L. A. (2008). Galphas-coupled receptor signaling actively down-regulates alpha4beta1-integrin affinity: a possible mechanism for cell de-adhesion. BMC Immunol, 9, 26. doi:10.1186/1471-2172-9-26

Cho, J. H., \& Feldman, M. (2015). Heterogeneity of autoimmune diseases: pathophysiologic insights from genetics and implications for new therapies. Nat Med, 21(7), 730-738. doi:10.1038/nm.3897

Chung, W.-S., Welsh, C. A., Barres, B. A., \& Stevens, B. (2015). Do glia drive synaptic and cognitive impairment in disease? Nat Neurosci, 18(11), 1539-1545. doi:10.1038/nn.4142

Ciabattini, A., Pettini, E., \& Medaglini, D. (2013). CD4(+) T Cell Priming as Biomarker to Study Immune Response to Preventive Vaccines. Frontiers in Immunology, 4, 421. doi:10.3389/fimmu.2013.00421

Codarri, L., Gyulveszi, G., Tosevski, V., Hesske, L., Fontana, A., Magnenat, L., . . Becher, B. (2011). RORgammat drives production of the cytokine GM-CSF in helper T cells, which is essential for the effector phase of autoimmune neuroinflammation. Nat Immunol, 12(6), 560-567. doi:10.1038/ni.2027

Cohen, T., Sundaresh, S., \& Levine, F. (2013). Antipsychotics activate the TGF $\beta$ pathway effector SMAD3. Mol Psychiatry, 18(3), 347-357. doi:10.1038/mp.2011.186

Cole, A. R. (2013). Glycogen synthase kinase 3 substrates in mood disorders and schizophrenia. Febs j, 280(21), 5213-5227. doi:10.1111/febs.12407

Compston, A., \& Coles, A. (2008). Multiple sclerosis. Lancet, 372(9648), 1502-1517. doi:10.1016/s0140-6736(08)61620-7

Connor, L. M., Tang, S. C., Camberis, M., Le Gros, G., \& Ronchese, F. (2014). Helminthconditioned dendritic cells prime CD4+ T cells to IL-4 production in vivo. J Immunol, 193(6), 2709-2717. doi:10.4049/jimmunol.1400374 
Constantinescu, C. S., Farooqi, N., O'Brien, K., \& Gran, B. (2011). Experimental autoimmune encephalomyelitis (EAE) as a model for multiple sclerosis (MS). British Journal of Pharmacology, 164(4), 1079-1106. doi:10.1111/j.1476-5381.2011.01302.x

Cua, D. J., Sherlock, J., Chen, Y., Murphy, C. A., Joyce, B., Seymour, B., . . Sedgwick, J. D. (2003). Interleukin-23 rather than interleukin-12 is the critical cytokine for autoimmune inflammation of the brain. Nature, 421(6924), 744-748. doi:10.1038/nature01355

De Sarno, P., Axtell, R. C., Raman, C., Roth, K. A., Alessi, D. R., \& Jope, R. S. (2008). Lithium prevents and ameliorates experimental autoimmune encephalomyelitis. $J$ Immunol, 181(1), 338-345.

Delgoffe, G. M., Kole, T. P., Zheng, Y., Zarek, P. E., Matthews, K. L., Xiao, B., . . Powell, J. D. (2009). The mTOR kinase differentially regulates effector and regulatory $\mathrm{T}$ cell lineage commitment. Immunity, 30(6), 832-844. doi:10.1016/j.immuni.2009.04.014

Dendrou, C. A., Fugger, L., \& Friese, M. A. (2015). Immunopathology of multiple sclerosis. Nat Rev Immunol, 15(9), 545-558. doi:10.1038/nri3871

http://www.nature.com/nri/journal/v15/n9/abs/nri3871.html - supplementary-information di Nuzzo, L., Orlando, R., Nasca, C., \& Nicoletti, F. (2014). Molecular pharmacodynamics of new oral drugs used in the treatment of multiple sclerosis. Drug Des Devel Ther, 8 , 555-568. doi:10.2147/dddt.s52428

Dogan, R. N., Elhofy, A., \& Karpus, W. J. (2008). Production of CCL2 by central nervous system cells regulates development of murine experimental autoimmune encephalomyelitis through the recruitment of TNF- and iNOS-expressing macrophages and myeloid dendritic cells. J Immunol, 180(11), 7376-7384.

Duda, P. W., Schmied, M. C., Cook, S. L., Krieger, J. I., \& Hafler, D. A. (2000). Glatiramer acetate (Copaxone) induces degenerate, Th2-polarized immune responses in patients with multiple sclerosis. J Clin Invest, 105(7), 967-976. doi:10.1172/jci8970

Dwivedi, Y., Rizavi, H. S., \& Pandey, G. N. (2002). Differential effects of haloperidol and clozapine on $[(3) \mathrm{H}] \mathrm{cAMP}$ binding, protein kinase A (PKA) activity, and mRNA and protein expression of selective regulatory and catalytic subunit isoforms of PKA in rat brain. J Pharmacol Exp Ther, 301(1), 197-209.

English, C., \& Aloi, J. J. (2015). New FDA-Approved Disease-Modifying Therapies for Multiple Sclerosis. Clinical Therapeutics, 37(4), 691-715. doi:http://dx.doi.org/10.1016/j.clinthera.2015.03.001 
Esposito, M., Ruffini, F., Bellone, M., Gagliani, N., Battaglia, M., Martino, G., \& Furlan, R. (2010). Rapamycin inhibits relapsing experimental autoimmune encephalomyelitis by both effector and regulatory T cells modulation. Journal of Neuroimmunology, 220(1-2), 52-63. doi:http://dx.doi.org/10.1016/j.jneuroim.2010.01.001

Feinstein, A., Freeman, J., \& Lo, A. C. Treatment of progressive multiple sclerosis: what works, what does not, and what is needed. The Lancet Neurology, 14(2), 194-207. doi:10.1016/S1474-4422(14)70231-5

Felts, P. A., Woolston, A. M., Fernando, H. B., Asquith, S., Gregson, N. A., Mizzi, O. J., \& Smith, K. J. (2005). Inflammation and primary demyelination induced by the intraspinal injection of lipopolysaccharide. Brain, 128(Pt 7), 1649-1666. doi:10.1093/brain/awh516

Feng, W. G., Wang, Y. B., Zhang, J. S., Wang, X. Y., Li, C. L., \& Chang, Z. L. (2002). cAMP elevators inhibit LPS-induced IL-12 p40 expression by interfering with phosphorylation of p38 MAPK in murine peritoneal macrophages. Cell Res, 12(5-6), 331-337. doi:10.1038/sj.cr.7290135

Ferguson, B., Matyszak, M. K., Esiri, M. M., \& Perry, V. H. (1997). Axonal damage in acute multiple sclerosis lesions. Brain, 120 ( Pt 3), 393-399.

Fillatreau, S., Sweenie, C. H., McGeachy, M. J., Gray, D., \& Anderton, S. M. (2002). B cells regulate autoimmunity by provision of IL-10. Nat Immunol, 3(10), 944-950. doi:10.1038/ni833

Fischer, M. T., Sharma, R., Lim, J. L., Haider, L., Frischer, J. M., Drexhage, J., . . . Lassmann, H. (2012). NADPH oxidase expression in active multiple sclerosis lesions in relation to oxidative tissue damage and mitochondrial injury. Brain, 135(Pt 3), 886899. doi:10.1093/brain/aws012

Fletcher, J. M., Lalor, S. J., Sweeney, C. M., Tubridy, N., \& Mills, K. H. G. (2010). T cells in multiple sclerosis and experimental autoimmune encephalomyelitis. Clinical and Experimental Immunology, 162(1), 1-11. doi:10.1111/j.1365-2249.2010.04143.x

Ford, A. L., Goodsall, A. L., Hickey, W. F., \& Sedgwick, J. D. (1995). Normal adult ramified microglia separated from other central nervous system macrophages by flow cytometric sorting. Phenotypic differences defined and direct ex vivo antigen presentation to myelin basic protein-reactive CD4+ T cells compared. J Immunol, 154(9), 4309-4321.

Forde, E. A., Dogan, R.-N. E., \& Karpus, W. J. (2011). CCR4 contributes to the pathogenesis of experimental autoimmune encephalomyelitis by regulating inflammatory 
macrophage function. Journal of Neuroimmunology, 236(1-2), 17-26.

doi:http://dx.doi.org/10.1016/j.jneuroim.2011.04.008

Frischer, J. M., Bramow, S., Dal-Bianco, A., Lucchinetti, C. F., Rauschka, H., Schmidbauer, M., .. . Lassmann, H. (2009). The relation between inflammation and neurodegeneration in multiple sclerosis brains. Brain, 132(5), 1175-1189. doi:10.1093/brain/awp070

Gajofatto, A., \& Benedetti, M. D. (2015). Treatment strategies for multiple sclerosis: When to start, when to change, when to stop? World Journal of Clinical Cases : WJCC, 3(7), 545-555. doi:10.12998/wjcc.v3.i7.545

Girard, J. P., Moussion, C., \& Forster, R. (2012). HEVs, lymphatics and homeostatic immune cell trafficking in lymph nodes. Nat Rev Immunol, 12(11), 762-773. doi:10.1038/nri3298

Gomez Perdiguero, E., Klapproth, K., Schulz, C., Busch, K., Azzoni, E., Crozet, L., . . . Rodewald, H. R. (2015). Tissue-resident macrophages originate from yolk-sacderived erythro-myeloid progenitors. Nature, 518(7540), 547-551. doi:10.1038/nature13989

Gourraud, P. A., Harbo, H. F., Hauser, S. L., \& Baranzini, S. E. (2012). The genetics of multiple sclerosis: an up-to-date review. Immunol Rev, 248(1), 87-103. doi:10.1111/j.1600-065X.2012.01134.X

Goverman, J. (2009). Autoimmune T cell responses in the central nervous system. Nat Rev Immunol, 9(6), 393-407.

Gran, B., Zhang, G. X., Yu, S., Li, J., Chen, X. H., Ventura, E. S., . . Rostami, A. (2002). IL-12p35-deficient mice are susceptible to experimental autoimmune encephalomyelitis: evidence for redundancy in the IL-12 system in the induction of central nervous system autoimmune demyelination. J Immunol, 169(12), 7104-7110.

Green, L., Zareie, P., Templeton, N., Keyzers, R., Conner, B., \& La Flamme, A. C. (2017). Enhanced disease reduction using clozapine, an atypical antipsychotic agent, and glatiramer acetate combination therapy in experimental autoimmune encephalomyelitis. Multiple Sclerosis Journal: Experimental, Translational and Clinical(Accepted).

Green, L. K., Zareie, P., Templeton, N., Keyzers, R. A., Connor, B., \& La Flamme, A. C. (2017). Enhanced disease reduction using clozapine, an atypical antipsychotic agent, and glatiramer acetate combination therapy in experimental autoimmune 
encephalomyelitis. Multiple Sclerosis Journal - Experimental, Translational and Clinical, 3(1), 2055217317698724. doi:10.1177/2055217317698724

Grover, S., Dutt, A., \& Avasthi, A. (2010). Indian research: Focus on clozapine. Indian Journal of Psychiatry, 52(2), 168-173. doi:10.4103/0019-5545.64592

Gurley, C., Nichols, J., Liu, S., Phulwani, N. K., Esen, N., \& Kielian, T. (2008). Microglia and Astrocyte Activation by Toll-Like Receptor Ligands: Modulation by PPARgamma Agonists. PPAR Res, 2008, 453120. doi:10.1155/2008/453120

Hansen, T. H., \& Bouvier, M. (2009). MHC class I antigen presentation: learning from viral evasion strategies. Nat Rev Immunol, 9(7), 503-513.

Harrington, L. E., Hatton, R. D., Mangan, P. R., Turner, H., Murphy, T. L., Murphy, K. M., \& Weaver, C. T. (2005). Interleukin 17-producing CD4+ effector T cells develop via a lineage distinct from the T helper type 1 and 2 lineages. Nat Immunol, 6(11), 1123 1132. doi:10.1038/ni1254

Heneka, M. T., Kummer, M. P., \& Latz, E. (2014). Innate immune activation in neurodegenerative disease. Nat Rev Immunol, 14(7), 463-477. doi:10.1038/nri3705

Heppner, F. L., Greter, M., Marino, D., Falsig, J., Raivich, G., Hovelmeyer, N., . . Aguzzi, A. (2005). Experimental autoimmune encephalomyelitis repressed by microglial paralysis. Nat Med, 11(2), 146-152. doi:10.1038/nm1177

Hickey, W. F., \& Kimura, H. (1988). Perivascular microglial cells of the CNS are bone marrow-derived and present antigen in vivo. Science, 239(4837), 290-292.

Hickman, S. E., Kingery, N. D., Ohsumi, T. K., Borowsky, M. L., Wang, L.-c., Means, T. K., \& El Khoury, J. (2013). The microglial sensome revealed by direct RNA sequencing. Nat Neurosci, 16(12), 1896-1905. doi:10.1038/nn.3554

http://www.nature.com/neuro/journal/v16/n12/abs/nn.3554.html - supplementary-information Ho, I. C., Tai, T.-S., \& Pai, S.-Y. (2009). GATA3 and the T-cell lineage: essential functions before and after T-helper-2-cell differentiation. Nature reviews. Immunology, 9(2), 125-135. doi:10.1038/nri2476

Hochmeister, S., Grundtner, R., Bauer, J., Engelhardt, B., Lyck, R., Gordon, G., . . . Lassmann, H. (2006). Dysferlin is a new marker for leaky brain blood vessels in multiple sclerosis. J Neuropathol Exp Neurol, 65(9), 855-865. doi:10.1097/01.jnen.0000235119.52311.16

Hong, J., Li, N., Zhang, X., Zheng, B., \& Zhang, J. Z. (2005). Induction of CD4+CD25+ regulatory $\mathrm{T}$ cells by copolymer-I through activation of transcription factor Foxp3. Proc Natl Acad Sci U S A, 102(18), 6449-6454. doi:10.1073/pnas.0502187102 
Hori, S., Nomura, T., \& Sakaguchi, S. (2003). Control of regulatory T cell development by the transcription factor Foxp3. Science, 299(5609), 1057-1061. doi:10.1126/science. 1079490

Hsieh, C.-S., Lee, H.-M., \& Lio, C.-W. J. (2012). Selection of regulatory T cells in the thymus. Nat Rev Immunol, 12(3), 157-167.

Hu, X., Leak, R. K., Shi, Y., Suenaga, J., Gao, Y., Zheng, P., \& Chen, J. (2015). Microglial and macrophage polarization-new prospects for brain repair. Nat Rev Neurol, 11(1), 56-64. doi:10.1038/nrneurol.2014.207

Hu, X., Zhou, H., Zhang, D., Yang, S., Qian, L., Wu, H. M., . . Hong, J. S. (2012). Clozapine protects dopaminergic neurons from inflammation-induced damage by inhibiting microglial overactivation. J Neuroimmune Pharmacol, 7(1), 187-201. doi:10.1007/s11481-011-9309-0

Huitinga, I., van Rooijen, N., de Groot, C. J., Uitdehaag, B. M., \& Dijkstra, C. D. (1990). Suppression of experimental allergic encephalomyelitis in Lewis rats after elimination of macrophages. J Exp Med, 172(4), 1025-1033.

Huseby, E. S., Huseby, P. G., Shah, S., Smith, R., \& Stadinski, B. D. (2012). Pathogenic CD8 T Cells in Multiple Sclerosis and Its Experimental Models. Frontiers in Immunology, 3, 64. doi:10.3389/fimmu.2012.00064

Ivanov, II, McKenzie, B. S., Zhou, L., Tadokoro, C. E., Lepelley, A., Lafaille, J. J., . . . Littman, D. R. (2006). The orphan nuclear receptor RORgammat directs the differentiation program of proinflammatory IL-17+ T helper cells. Cell, 126(6), 11211133. doi:10.1016/j.cell.2006.07.035

Iwasaki, A., \& Medzhitov, R. (2015). Control of adaptive immunity by the innate immune system. Nat Immunol, 16(4), 343-353. doi:10.1038/ni.3123

Jeffery, D. R., \& Herndon, R. (2004). Review of mitoxantrone in the treatment of multiple sclerosis. Neurology, 63(12 Suppl 6), S19-24.

Jiang, Z., Jiang, J. X., \& Zhang, G. X. (2014). Macrophages: a double-edged sword in experimental autoimmune encephalomyelitis. Immunol Lett, 160(1), 17-22. doi:10.1016/j.imlet.2014.03.006

Jope, R. S., \& Roh, M.-S. (2006). Glycogen Synthase Kinase-3 (GSK3) in Psychiatric Diseases and Therapeutic Interventions. Current drug targets, 7(11), 1421-1434.

Juedes, A. E., Hjelmström, P., Bergman, C. M., Neild, A. L., \& Ruddle, N. H. (2000). Kinetics and Cellular Origin of Cytokines in the Central Nervous System: Insight into Mechanisms of Myelin Oligodendrocyte Glycoprotein-Induced Experimental 
Autoimmune Encephalomyelitis. The Journal of Immunology, 164(1), 419-426. doi:10.4049/jimmunol.164.1.419

Kageyama, T., Nakamura, M., Matsuo, A., Yamasaki, Y., Takakura, Y., Hashida, M., . . Shimohama, S. (2000). The 4F2hc/LAT1 complex transports 1-DOPA across the blood-brain barrier. Brain Res, 879(1-2), 115-121. doi:http://dx.doi.org/10.1016/S0006-8993(00)02758-X

Kapuscinski, J., \& Darzynkiewicz, Z. (1986). Relationship between the pharmacological activity of antitumor drugs Ametantrone and mitoxantrone (Novatrone) and their ability to condense nucleic acids. Proceedings of the National Academy of Sciences, 83(17), 6302-6306.

Karagkouni, A., Alevizos, M., \& Theoharides, T. C. (2013). Effect of stress on brain inflammation and multiple sclerosis. Autoimmun Rev, 12(10), 947-953. doi:10.1016/j.autrev.2013.02.006

Kato, T., Monji, A., Hashioka, S., \& Kanba, S. (2007). Risperidone significantly inhibits interferon-gamma-induced microglial activation in vitro. Schizophr Res, 92(1-3), 108115. doi:10.1016/j.schres.2007.01.019

Kato, T., Monji, A., Hashioka, S., \& Kanba, S. (2007). Risperidone significantly inhibits interferon- $\gamma$-induced microglial activation in vitro. Schizophr Res, 92(1-3), 108-115. doi:http://dx.doi.org/10.1016/j.schres.2007.01.019

Kent, S. J., Karlik, S. J., Cannon, C., Hines, D. K., Yednock, T. A., Fritz, L. C., \& Horner, H. C. (1995). A monoclonal antibody to $\alpha 4$ integrin suppresses and reverses active experimental allergic encephalomyelitis. Journal of Neuroimmunology, 58(1), 1-10. doi:http://dx.doi.org/10.1016/0165-5728(94)00165-K

Kettenmann, H., Hanisch, U. K., Noda, M., \& Verkhratsky, A. (2011). Physiology of microglia. Physiol Rev, 91(2), 461-553. doi:10.1152/physrev.00011.2010

Kim, C., \& Tse, H. Y. (1993). Adoptive transfer of murine experimental autoimmune encephalomyelitis in SJL.Thy-1 congenic mouse strains. J Neuroimmunol, 46(1-2), 129-136.

Kim, H. J., Ifergan, I., Antel, J. P., Seguin, R., Duddy, M., Lapierre, Y., . . Bar-Or, A. (2004). Type 2 monocyte and microglia differentiation mediated by glatiramer acetate therapy in patients with multiple sclerosis. J Immunol, 172(11), 7144-7153.

Kim, H. Y., DeKruyff, R. H., \& Umetsu, D. T. (2010). The many paths to asthma: phenotype shaped by innate and adaptive immunity. Nat Immunol, 11(7), 577-584. 
Kim, J. M., Rasmussen, J. P., \& Rudensky, A. Y. (2007). Regulatory T cells prevent catastrophic autoimmunity throughout the lifespan of mice. Nat Immunol, 8(2), 191197. doi:10.1038/ni1428

Kim, W. S., Kim, J.-S., Cha, S. B., Kim, H., Kwon, K. W., Kim, S. J., . . Shin, S. J. (2016). Mycobacterium tuberculosis Rv3628 drives Th1-type T cell immunity via TLR2mediated activation of dendritic cells and displays vaccine potential against the hypervirulent Beijing K strain. Oncotarget, 7(18), 24962-24982. doi:10.18632/oncotarget.8771

Kim, Y. K., Suh, I. B., Kim, H., Han, C. S., Lim, C. S., Choi, S. H., \& Licinio, J. (2002). The plasma levels of interleukin-12 in schizophrenia, major depression, and bipolar mania: effects of psychotropic drugs. Mol Psychiatry, 7(10), 1107-1114. doi:10.1038/sj.mp.4001084

Klein, L., Kyewski, B., Allen, P. M., \& Hogquist, K. A. (2014). Positive and negative selection of the T cell repertoire: what thymocytes see and don't see. Nature reviews. Immunology, 14(6), 377-391. doi:10.1038/nri3667

Ko, R., \& Lee, S. Y. (2016). Glycogen synthase kinase $3 \beta$ in Toll-like receptor signaling. BMB Reports, 49(6), 305-310. doi:10.5483/BMBRep.2016.49.6.059

Kobayashi, K., Imagama, S., Ohgomori, T., Hirano, K., Uchimura, K., Sakamoto, K., . . . Kadomatsu, K. (2013). Minocycline selectively inhibits M1 polarization of microglia. Cell Death Dis, 4, e525. doi:10.1038/cddis.2013.54

Kohm, A. P., Carpentier, P. A., Anger, H. A., \& Miller, S. D. (2002). Cutting Edge: CD4+CD25+ Regulatory T Cells Suppress Antigen-Specific Autoreactive Immune Responses and Central Nervous System Inflammation During Active Experimental Autoimmune Encephalomyelitis. The Journal of Immunology, 169(9), 4712-4716. doi:10.4049/jimmunol.169.9.4712

Kopf, H., de la Rosa, G. M., Howard, O., \& Chen, X. (2007). Rapamycin inhibits differentiation of Th17 cells and promotes generation of FoxP3+ T regulatory cells. International immunopharmacology, 7(13), 1819-1824. doi:10.1016/j.intimp.2007.08.027

Koutrolos, M., Berer, K., Kawakami, N., Wekerle, H., \& Krishnamoorthy, G. (2014). Treg cells mediate recovery from EAE by controlling effector $\mathrm{T}$ cell proliferation and motility in the CNS. Acta Neuropathologica Communications, 2, 163. doi:10.1186/s40478-014-0163-1 
Kouwenhoven, M., Teleshova, N., Özenci, V., Press, R., \& Link, H. (2001). Monocytes in multiple sclerosis: phenotype and cytokine profile. Journal of Neuroimmunology, 112(1-2), 197-205. doi:http://dx.doi.org/10.1016/S0165-5728(00)00396-9

Krausgruber, T., Blazek, K., Smallie, T., Alzabin, S., Lockstone, H., Sahgal, N., . . Udalova, I. A. (2011). IRF5 promotes inflammatory macrophage polarization and TH1-TH17 responses. Nat Immunol, 12(3), 231-238. doi:10.1038/ni.1990

Kreutzberg, G. W. (1996). Microglia: a sensor for pathological events in the CNS. Trends in Neurosciences, 19(8), 312-318. doi:http://dx.doi.org/10.1016/0166-2236(96)10049-7

Kurosaki, T., Kometani, K., \& Ise, W. (2015). Memory B cells. Nat Rev Immunol, 15(3), 149-159. doi:10.1038/nri3802

La Flamme, A. C., Kharkrang, M., Stone, S., Mirmoeini, S., Chuluundorj, D., \& Kyle, R. (2012). Type II-Activated Murine Macrophages Produce IL-4. PLoS One, 7(10), e46989. doi:10.1371/journal.pone.0046989

Land, W. G. (2015). The Role of Damage-Associated Molecular Patterns in Human Diseases: Part I - Promoting inflammation and immunity. Sultan Qaboos University Medical Journal, 15(1), e9-e21.

Laskaris, L. E., Di Biase, M. A., Everall, I., Chana, G., Christopoulos, A., Skafidas, E., . . Pantelis, C. (2016). Microglial activation and progressive brain changes in schizophrenia. Br J Pharmacol, 173(4), 666-680. doi:10.1111/bph.13364

Lassmann, H., van Horssen, J., \& Mahad, D. (2012). Progressive multiple sclerosis: pathology and pathogenesis. Nat Rev Neurol, 8(11), 647-656. doi:10.1038/nrneurol.2012.168

Lazarevic, V., Glimcher, L. H., \& Lord, G. M. (2013). T-bet: a bridge between innate and adaptive immunity. Nat Rev Immunol, 13(11), 777-789. doi:10.1038/nri3536

LeBien, T. W., \& Tedder, T. F. (2008). B lymphocytes: how they develop and function. Blood, 112(5), 1570-1580. doi:10.1182/blood-2008-02-078071

Lenschow, D. J., Walunas, T. L., \& Bluestone, J. A. (1996). CD28/B7 system of T cell costimulation. Annu Rev Immunol, 14, 233-258. doi:10.1146/annurev.immunol.14.1.233

Leucht, S., Wahlbeck, K., Hamann, J., \& Kissling, W. (2003). New generation antipsychotics versus low-potency conventional antipsychotics: a systematic review and metaanalysis. Lancet, 361(9369), 1581-1589. doi:10.1016/s0140-6736(03)13306-5

Levite, M. (2016). Dopamine and T cells: dopamine receptors and potent effects on T cells, dopamine production in $\mathrm{T}$ cells, and abnormalities in the dopaminergic system in $\mathrm{T}$ 
cells in autoimmune, neurological and psychiatric diseases. Acta Physiol (Oxf), 216(1), 42-89. doi:10.1111/apha.12476

Li, X., Rosborough, K. M., Friedman, A. B., Zhu, W., \& Roth, K. A. (2007). Regulation of mouse brain glycogen synthase kinase-3 by atypical antipsychotics. Int $J$ Neuropsychopharmacol, 10(1), 7-19. doi:10.1017/s1461145706006547

Lieber, M. R. (1991). Site-specific recombination in the immune system. Faseb j, 5(14), 2934-2944.

Liu, F., Guo, X., Wu, R., Ou, J., Zheng, Y., Zhang, B., . . Zhao, J. (2014). Minocycline supplementation for treatment of negative symptoms in early-phase schizophrenia: a double blind, randomized, controlled trial. Schizophr Res, 153(1-3), 169-176. doi:10.1016/j.schres.2014.01.011

Lobb, R. R., \& Hemler, M. E. (1994). The pathophysiologic role of alpha 4 integrins in vivo. J Clin Invest, 94(5), 1722-1728. doi:10.1172/JCI117519

Lotharius, J., \& Brundin, P. (2002). Pathogenesis of parkinson's disease: dopamine, vesicles and [alpha]-synuclein. Nat Rev Neurosci, 3(12), 932-942.

Lu, F. W., Yasutomo, K., Goodman, G. B., McHeyzer-Williams, L. J., McHeyzer-Williams, M. G., Germain, R. N., \& Ashwell, J. D. (2000). Thymocyte resistance to glucocorticoids leads to antigen-specific unresponsiveness due to "holes" in the T cell repertoire. Immunity, 12(2), 183-192.

Lublin, F. D., \& Reingold, S. C. (1996). Defining the clinical course of multiple sclerosis: results of an international survey. National Multiple Sclerosis Society (USA) Advisory Committee on Clinical Trials of New Agents in Multiple Sclerosis. Neurology, 46(4), 907-911.

Luccarini, I., Ballerini, C., Biagioli, T., Biamonte, F., Bellucci, A., Rosi, M. C., . . . Casamenti, F. (2008). Combined treatment with atorvastatin and minocycline suppresses severity of EAE. Exp Neurol, 211(1), 214-226. doi:http://dx.doi.org/10.1016/j.expneurol.2008.01.022

Luning Prak, E. T., Monestier, M., \& Eisenberg, R. A. (2011). B cell receptor editing in tolerance and autoimmunity. Annals of the New York Academy of Sciences, 1217, 96121. doi:10.1111/j.1749-6632.2010.05877.x

Maceyka, M., \& Spiegel, S. (2014). Sphingolipid metabolites in inflammatory disease. Nature, 510(7503), 58-67. doi:10.1038/nature13475 
Mahad, D. H., Trapp, B. D., \& Lassmann, H. Pathological mechanisms in progressive multiple sclerosis. The Lancet Neurology, 14(2), 183-193. doi:10.1016/S14744422(14)70256-X

Maloy, K. J., \& Powrie, F. (2001). Regulatory T cells in the control of immune pathology. Nat Immunol, 2(9), 816-822.

Mannara, F., Valente, T., Saura, J., Graus, F., Saiz, A., \& Moreno, B. (2012). Passive Experimental Autoimmune Encephalomyelitis in C57BL/6 with MOG: Evidence of Involvement of B Cells. PLoS One, 7(12), e52361. doi:10.1371/journal.pone.0052361

Marckmann, S., Wiesemann, E., Hilse, R., Trebst, C., Stangel, M., \& Windhagen, A. (2004). Interferon- $\beta$ up-regulates the expression of co-stimulatory molecules CD80, CD86 and CD40 on monocytes: significance for treatment of multiple sclerosis. Clinical and Experimental Immunology, 138(3), 499-506. doi:10.1111/j.1365-2249.2004.02624.x

Martin, M., Rehani, K., Jope, R. S., \& Michalek, S. M. (2005). Toll-like receptor-mediated cytokine production is differentially regulated by glycogen synthase kinase 3 . Nat Immunol, 6(8), 777-784. doi:10.1038/ni1221

McCarthy, D. P., Richards, M. H., \& Miller, S. D. (2012). Mouse Models of Multiple Sclerosis: Experimental Autoimmune Encephalomyelitis and Theiler's Virus-Induced Demyelinating Disease. Methods in molecular biology (Clifton, N.J.), 900, 381-401. doi:10.1007/978-1-60761-720-4_19

McEver, R. P. (2015). Selectins: initiators of leucocyte adhesion and signalling at the vascular wall. Cardiovasc Res, 107(3), 331-339. doi:10.1093/cvr/cvv154

McGeachy, M. J., Stephens, L. A., \& Anderton, S. M. (2005). Natural recovery and protection from autoimmune encephalomyelitis: contribution of CD4+CD25+ regulatory cells within the central nervous system. J Immunol, 175(5), 3025-3032.

McKenna, F., McLaughlin, P. J., Lewis, B. J., Sibbring, G. C., Cummerson, J. A., BowenJones, D., \& Moots, R. J. (2002). Dopamine receptor expression on human T- and Blymphocytes, monocytes, neutrophils, eosinophils and NK cells: a flow cytometric study. J Neuroimmunol, 132(1-2), 34-40.

Mei, F., Guo, S., He, Y., Wang, L., Wang, H., Niu, J., . . Xiao, L. (2012). Quetiapine, an Atypical Antipsychotic, Is Protective against Autoimmune-Mediated Demyelination by Inhibiting Effector T Cell Proliferation. PLoS One, 7(8), e42746. doi:10.1371/journal.pone.0042746 
Melief, J., Koning, N., Schuurman, K. G., Van De Garde, M. D. B., Smolders, J., Hoek, R. M., . . . Huitinga, I. (2012). Phenotyping primary human microglia: Tight regulation of LPS responsiveness. Glia, 60(10), 1506-1517. doi:10.1002/glia.22370

Melnikov, M., Belousova, O., Murugin, V., Pashenkov, M., \& Воуко, А. (2016). The role of dopamine in modulation of Th-17 immune response in multiple sclerosis. Journal of Neuroimmunology, 292, 97-101. doi:http://dx.doi.org/10.1016/j.jneuroim.2016.01.020

Mendels, J., Stinnett, J. L., Burns, D., \& Frazer, A. (1975). Amine precursors and depression. Arch Gen Psychiatry, 32(1), 22-30.

Merkler, D., Ernsting, T., Kerschensteiner, M., Bruck, W., \& Stadelmann, C. (2006). A new focal EAE model of cortical demyelination: multiple sclerosis-like lesions with rapid resolution of inflammation and extensive remyelination. Brain, 129(Pt 8), 1972-1983. doi:10.1093/brain/awl135

Mildner, A., Mack, M., Schmidt, H., Bruck, W., Djukic, M., Zabel, M. D., . . Prinz, M. (2009). CCR2+Ly-6Chi monocytes are crucial for the effector phase of autoimmunity in the central nervous system. Brain, 132(Pt 9), 2487-2500. doi:10.1093/brain/awp144

Miles, J. J., Douek, D. C., \& Price, D. A. (2011). Bias in the [alpha][beta] T-cell repertoire: implications for disease pathogenesis and vaccination. Immunol Cell Biol, 89(3), 375387.

Miller, B. J., Buckley, P., Seabolt, W., Mellor, A., \& Kirkpatrick, B. (2011). Meta-Analysis of Cytokine Alterations in Schizophrenia: Clinical Status and Antipsychotic Effects. Biological Psychiatry, 70(7), 663-671. doi:http://dx.doi.org/10.1016/j.biopsych.2011.04.013

Miller, S. D., Karpus, W. J., \& Davidson, T. S. (2007). Experimental Autoimmune Encephalomyelitis in the Mouse. Current protocols in immunology / edited by John E. Coligan ... [et al.], CHAPTER, Unit-15.11. doi:10.1002/0471142735.im1501s77

Milo, I., Sapoznikov, A., Kalchenko, V., Tal, O., Krauthgamer, R., van Rooijen, N., . . Shakhar, G. (2013). Dynamic imaging reveals promiscuous crosspresentation of blood-borne antigens to naive CD8+ T cells in the bone marrow. Blood, 122(2), 193208. doi:10.1182/blood-2012-01-401265

Miron, V. E., Boyd, A., Zhao, J.-W., Yuen, T. J., Ruckh, J. M., Shadrach, J. L., . . ffrenchConstant, C. (2013). M2 microglia/macrophages drive oligodendrocyte differentiation during CNS remyelination. Nature neuroscience, 16(9), 1211-1218. doi:10.1038/nn.3469 
Mishra, M. K., \& Yong, V. W. (2016). Myeloid cells - targets of medication in multiple sclerosis. Nat Rev Neurol, 12(9), 539-551. doi:10.1038/nrneurol.2016.110

Mishra, M. K., \& Yong, V. W. (2016). Myeloid cells [mdash] targets of medication in multiple sclerosis. Nat Rev Neurol, 12(9), 539-551. doi:10.1038/nrneurol.2016.110

Miyaoka, T., Yasukawa, R., Yasuda, H., Hayashida, M., Inagaki, T., \& Horiguchi, J. (2008). Minocycline as adjunctive therapy for schizophrenia: an open-label study. Clin Neuropharmacol, 31(5), 287-292. doi:10.1097/WNF.0b013e3181593d45

Mizutani, M., Pino, P. A., Saederup, N., Charo, I. F., Ransohoff, R. M., \& Cardona, A. E. (2012). The fractalkine receptor but not CCR2 is present on microglia from embryonic development throughout adulthood. J Immunol, 188(1), 29-36. doi:10.4049/jimmunol.1100421

Monji, A., Kato, T., \& Kanba, S. (2009). Cytokines and schizophrenia: Microglia hypothesis of schizophrenia. Psychiatry Clin Neurosci, 63(3), 257-265.

Monji, A., Kato, T. A., Mizoguchi, Y., Horikawa, H., Seki, Y., Kasai, M., . . Kanba, S. (2013). Neuroinflammation in schizophrenia especially focused on the role of microglia. Prog Neuropsychopharmacol Biol Psychiatry, 42, 115-121. doi:10.1016/j.pnpbp.2011.12.002

Montero, E., Nussbaum, G., Kaye, J. F., Perez, R., Lage, A., Ben-Nun, A., \& Cohen, I. R. (2004). Regulation of experimental autoimmune encephalomyelitis by CD4+, CD25+ and CD8+ T cells: analysis using depleting antibodies. $J$ Autoimmun, 23(1), 1-7. doi:10.1016/j.jaut.2004.05.001

Mosmann, T. R., \& Coffman, R. L. (1989). TH1 and TH2 cells: different patterns of lymphokine secretion lead to different functional properties. Annu Rev Immunol, 7, 145-173. doi:10.1146/annurev.iy.07.040189.001045

Mosser, D. M., \& Zhang, X. (2008). Activation of Murine Macrophages. Current protocols in immunology / edited by John E. Coligan ... [et al.], CHAPTER, Unit. doi:10.1002/0471142735.im1402s83

Müller, N., Weidinger, E., Leitner, B., \& Schwarz, M. J. (2015). The role of inflammation in schizophrenia. Frontiers in Neuroscience, 9, 372. doi:10.3389/fnins.2015.00372

Neefjes, J., Jongsma, M. L., Paul, P., \& Bakke, O. (2011). Towards a systems understanding of MHC class I and MHC class II antigen presentation. Nat Rev Immunol, 11(12), 823-836. doi:10.1038/nri3084

Nemazee, D. (2006). Receptor editing in lymphocyte development and central tolerance. Nat Rev Immunol, 6(10), 728-740. 
Neuhaus, O., Farina, C., Yassouridis, A., Wiendl, H., Then Bergh, F., Dose, T., .. . Hohlfeld, R. (2000). Multiple sclerosis: Comparison of copolymer-1- reactive T cell lines from treated and untreated subjects reveals cytokine shift from $\mathrm{T}$ helper 1 to $\mathrm{T}$ helper 2 cells. Proceedings of the National Academy of Sciences, 97(13), 7452-7457. doi:10.1073/pnas.97.13.7452

Neuhaus, O., Wiendl, H., Kieseier, B. C., Archelos, J. J., Hemmer, B., Stüve, O., \& Hartung, H.-P. (2005). Multiple sclerosis: Mitoxantrone promotes differential effects on immunocompetent cells in vitro. Journal of Neuroimmunology, 168(1-2), 128-137. doi:http://dx.doi.org/10.1016/j.jneuroim.2005.01.024

Nimmerjahn, A., Kirchhoff, F., \& Helmchen, F. (2005). Resting microglial cells are highly dynamic surveillants of brain parenchyma in vivo. Science, 308(5726), 1314-1318. doi:10.1126/science.1110647

O'Garra, A., Barrat, F. J., Castro, A. G., Vicari, A., \& Hawrylowicz, C. (2008). Strategies for use of IL-10 or its antagonists in human disease. Immunol Rev, 223, 114-131. doi:10.1111/j.1600-065X.2008.00635.X

O'Sullivan, D., Green, L., Stone, S., Zareie, P., Kharkrang, M., Fong, D., . . La Flamme, A. C. (2014). Treatment with the antipsychotic agent, risperidone, reduces disease severity in experimental autoimmune encephalomyelitis. PLoS One, 9(8), e104430. doi:10.1371/journal.pone.0104430

O'Sullivan, D., Miller, J. H., Northcote, P. T., \& La Flamme, A. C. (2013). Microtubulestabilizing agents delay the onset of EAE through inhibition of migration. Immunol Cell Biol, 91(9), 583-592. doi:10.1038/icb.2013.47

Olianas, M. C., Maullu, C., \& Onali, P. (1999). Mixed Agonist-Antagonist Properties of Clozapine at Different Human Cloned Muscarinic Receptor Subtypes Expressed in Chinese Hamster Ovary Cells. Neuropsychopharmacology, 20(3), 263-270.

Pacheco, R., Contreras, F., \& Zouali, M. (2014). The Dopaminergic System in Autoimmune Diseases. Frontiers in Immunology, 5, 117. doi:10.3389/fimmu.2014.00117

Pacheco, R., Prado, C. E., Barrientos, M. J., \& Bernales, S. (2009). Role of dopamine in the physiology of T-cells and dendritic cells. J Neuroimmunol, 216(1-2), 8-19. doi:10.1016/j.jneuroim.2009.07.018

Pacheco, R., Riquelme, E., \& Kalergis, A. M. (2010). Emerging evidence for the role of neurotransmitters in the modulation of $\mathrm{T}$ cell responses to cognate ligands. Cent Nerv Syst Agents Med Chem, 10(1), 65-83. 
Panariello, F., Perruolo, G., Cassese, A., Giacco, F., Botta, G., Barbagallo, A. P., . . de Bartolomeis, A. (2012). Clozapine impairs insulin action by up-regulating Akt phosphorylation and Ped/Pea-15 protein abundance. J Cell Physiol, 227(4), 14851492. doi:10.1002/jcp.22864

Panariello, F., Perruolo, G., Cassese, A., Giacco, F., Botta, G., Barbagallo, A. P. M., . . de Bartolomeis, A. (2012). Clozapine Impairs Insulin Action by Up-Regulating Akt Phosphorylation and Ped/Pea-15 Protein Abundance. J Cell Physiol, 227(4), 14851492. doi:10.1002/jcp.22864

Papadopoulos, D., Pham-Dinh, D., \& Reynolds, R. (2006). Axon loss is responsible for chronic neurological deficit following inflammatory demyelination in the rat. Exp Neurol, 197(2), 373-385. doi:10.1016/j.expneurol.2005.10.033

Pardridge, W. M. (2005a). The blood-brain barrier: bottleneck in brain drug development. NeuroRx, 2(1), 3-14. doi:10.1602/neurorx.2.1.3

Pardridge, W. M. (2005b). Molecular biology of the blood-brain barrier. Mol Biotechnol, 30(1), 57-70. doi:10.1385/mb:30:1:057

Patani, R., Balaratnam, M., Vora, A., \& Reynolds, R. (2007). Remyelination can be extensive in multiple sclerosis despite a long disease course. Neuropathol Appl Neurobiol, 33(3), 277-287. doi:10.1111/j.1365-2990.2007.00805.x

Patel, A. R., Ritzel, R., McCullough, L. D., \& Liu, F. (2013). Microglia and ischemic stroke: a double-edged sword. International Journal of Physiology, Pathophysiology and Pharmacology, 5(2), 73-90.

Paul, W. E., \& Zhu, J. (2010). How are TH2-type immune responses initiated and amplified? Nat Rev Immunol, 10(4), 225-235.

Perry, V. H., Nicoll, J. A. R., \& Holmes, C. (2010). Microglia in neurodegenerative disease. Nat Rev Neurol, 6(4), 193-201.

Phillips, J. M., Parish, N. M., Raine, T., Bland, C., Sawyer, Y., De La Pena, H., \& Cooke, A. (2009). Type 1 diabetes development requires both CD4+ and CD8+ T cells and can be reversed by non-depleting antibodies targeting both T cell populations. Rev Diabet Stud, 6(2), 97-103. doi:10.1900/rds.2009.6.97

Pinkse, G. G., Tysma, O. H., Bergen, C. A., Kester, M. G., Ossendorp, F., van Veelen, P. A., ... Roep, B. O. (2005). Autoreactive CD8 T cells associated with beta cell destruction in type 1 diabetes. Proc Natl Acad Sci U S A, 102(51), 18425-18430. doi:10.1073/pnas.0508621102 
Platten, M., \& Steinman, L. (2005). Multiple sclerosis: trapped in deadly glue. Nat Med, 11(3), 252-253. doi:10.1038/nm0305-252

Polman, C. H., O'Connor, P. W., Havrdova, E., Hutchinson, M., Kappos, L., Miller, D. H., . . . Sandrock, A. W. (2006). A Randomized, Placebo-Controlled Trial of Natalizumab for Relapsing Multiple Sclerosis. New England Journal of Medicine, 354(9), 899-910. doi:10.1056/NEJMoa044397

Ponomarev, E. D., Shriver, L. P., Maresz, K., \& Dittel, B. N. (2005). Microglial cell activation and proliferation precedes the onset of CNS autoimmunity. $J$ Neurosci Res, 81(3), 374-389. doi:10.1002/jnr.20488

Ponomarev, E. D., Shriver, L. P., Maresz, K., Pedras-Vasconcelos, J., Verthelyi, D., \& Dittel, B. N. (2007). GM-CSF production by autoreactive T cells is required for the activation of microglial cells and the onset of experimental autoimmune encephalomyelitis. J Immunol, 178(1), 39-48.

Ponomarev, E. D., Veremeyko, T., Barteneva, N., Krichevsky, A. M., \& Weiner, H. L. (2011). MicroRNA-124 promotes microglia quiescence and suppresses EAE by deactivating macrophages via the C/EBP-[alpha]-PU.1 pathway. Nat Med, 17(1), 6470. doi:http://www.nature.com/nm/journal/v17/n1/abs/nm.2266.html supplementary-information

Pozzi, L., Hakansson, K., Usiello, A., Borgkvist, A., Lindskog, M., Greengard, P., \& Fisone, G. (2003). Opposite regulation by typical and atypical anti-psychotics of ERK1/2, CREB and Elk-1 phosphorylation in mouse dorsal striatum. J Neurochem, 86(2), 451459.

Prineas, J. W., Kwon, E. E., Cho, E. S., Sharer, L. R., Barnett, M. H., Oleszak, E. L., . . Morgan, B. P. (2001). Immunopathology of secondary-progressive multiple sclerosis. Ann Neurol, 50(5), 646-657.

Rački, V., Petrić, D., Kučić, N., Gržeta, N., Jurdana, K., \& Rončević-Gržeta, I. Cortical gray matter loss in schizophrenia: Could microglia be the culprit? Medical Hypotheses, 88, 18-21. doi:10.1016/j.mehy.2015.12.021

Raghavendra, P. B., Lee, E., \& Parameswaran, N. (2014). Regulation of macrophage biology by Lithium: A new look at an old drug. J Neuroimmune Pharmacol, 9(3), 277-284. doi:10.1007/s11481-013-9516-y

Ransohoff, R. M., \& Brown, M. A. (2012). Innate immunity in the central nervous system. $J$ Clin Invest, 122(4), 1164-1171. doi:10.1172/JCI58644 
Ridge, S. C., Sloboda, A. E., McReynolds, R. A., Levine, S., Oronsky, A. L., \& Kerwar, S. S. (1985). Suppression of experimental allergic encephalomyelitis by mitoxantrone. Clin Immunol Immunopathol, 35(1), 35-42.

Roche, P. A., \& Furuta, K. (2015). The ins and outs of MHC class II-mediated antigen processing and presentation. Nat Rev Immunol, 15(4), 203-216. doi:10.1038/nri3818

Roh, M. S., Seo, M. S., Kim, Y., Kim, S. H., Jeon, W. J., Ahn, Y. M., . . Kim, Y. S. (2007). Haloperidol and clozapine differentially regulate signals upstream of glycogen synthase kinase 3 in the rat frontal cortex. Exp Mol Med, 39(3), 353-360. doi:10.1038/emm.2007.39

Roman, A., Kusmierczyk, J., Klimek, E., Rogoz, Z., \& Nalepa, I. (2012). Effects of coadministration of fluoxetine and risperidone on properties of peritoneal and pleural macrophages in rats subjected to the forced swimming test. Pharmacol Rep, 64(6), $1368-1380$.

Rosser, E. C., \& Mauri, C. (2015). Regulatory B cells: origin, phenotype, and function. Immunity, 42(4), 607-612. doi:10.1016/j.immuni.2015.04.005

Sacre, S., Medghalchi, M., Gregory, B., Brennan, F., \& Williams, R. (2010). Fluoxetine and citalopram exhibit potent antiinflammatory activity in human and murine models of rheumatoid arthritis and inhibit toll-like receptors. Arthritis Rheum, 62(3), 683-693. doi:10.1002/art.27304

Sakaguchi, S., Sakaguchi, N., Shimizu, J., Yamazaki, S., Sakihama, T., Itoh, M., . . . Takahashi, T. (2001). Immunologic tolerance maintained by CD25+CD4+ regulatory T cells: their common role in controlling autoimmunity, tumor immunity, and transplantation tolerance. Immunol Rev, 182, 18-32.

Schatz, D. G., \& Ji, Y. (2011). Recombination centres and the orchestration of V(D)J recombination. Nat Rev Immunol, 11(4), 251-263.

Schizophrenia Working Group of the Psychiatric Genomics, C. (2014). Biological insights from 108 schizophrenia-associated genetic loci. Nature, 511(7510), 421-427. doi:10.1038/nature13595

http://www.nature.com/nature/journal/v511/n7510/abs/nature13595.html - supplementaryinformation

Schmid, C. L., Streicher, J. M., Meltzer, H. Y., \& Bohn, L. M. (2014). Clozapine acts as an agonist at serotonin 2A receptors to counter MK-801-induced behaviors through a betaarrestin2-independent activation of Akt. Neuropsychopharmacology, 39(8), 19021913. doi:10.1038/npp.2014.38 
Schmidt, R. H., Jokinen, J. D., Massey, V. L., Falkner, K. C., Shi, X., Yin, X., . . Arteel, G. E. (2013). Olanzapine Activates Hepatic Mammalian Target of Rapamycin: New Mechanistic Insight into Metabolic Dysregulation with Atypical Antipsychotic Drugs. Journal of Pharmacology and Experimental Therapeutics, 347(1), 126-135. doi:10.1124/jpet.113.207621

Schmitt, E. G., \& Williams, C. B. (2013). Generation and Function of Induced Regulatory T Cells. Frontiers in Immunology, 4, 152. doi:10.3389/fimmu.2013.00152

Sedgwick, J. D., Schwender, S., Imrich, H., Dorries, R., Butcher, G. W., \& ter Meulen, V. (1991). Isolation and direct characterization of resident microglial cells from the normal and inflamed central nervous system. Proc Natl Acad Sci U S A, 88(16), 74387442.

Seidell, J. C. (2010). Waist circumference and waist/hip ratio in relation to all-cause mortality, cancer and sleep apnea. Eur J Clin Nutr, 64(1), 35-41. doi:10.1038/ejen.2009.71

Setiady, Y. Y., Coccia, J. A., \& Park, P. U. (2010). In vivo depletion of CD4+FOXP3+ Treg cells by the PC61 anti-CD25 monoclonal antibody is mediated by Fc $\gamma$ RIII+ phagocytes. Eur J Immunol, 40(3), 780-786. doi:10.1002/eji.200939613

Shamri, R., Grabovsky, V., Feigelson, S. W., Dwir, O., Van Kooyk, Y., \& Alon, R. (2002). Chemokine stimulation of lymphocyte alpha 4 integrin avidity but not of leukocyte function-associated antigen-1 avidity to endothelial ligands under shear flow requires cholesterol membrane rafts. $J$ Biol Chem, 277(42), 40027-40035. doi:10.1074/jbc.M206806200

Sharif, O., Bolshakov, V. N., Raines, S., Newham, P., \& Perkins, N. D. (2007). Transcriptional profiling of the LPS induced NF- $\kappa \mathrm{B}$ response in macrophages. $B M C$ Immunology, 8(1), 1. doi:10.1186/1471-2172-8-1

Shechter, R., Miller, O., Yovel, G., Rosenzweig, N., London, A., Ruckh, J., . . Schwartz, M. (2013). Recruitment of beneficial M2 macrophages to injured spinal cord is orchestrated by remote brain choroid plexus. Immunity, 38(3), 555-569. doi:10.1016/j.immuni.2013.02.012

Shields, S. A., Gilson, J. M., Blakemore, W. F., \& Franklin, R. J. (1999). Remyelination occurs as extensively but more slowly in old rats compared to young rats following gliotoxin-induced CNS demyelination. Glia, 28(1), 77-83.

Shin, S. Y., Choi, B. H., Ko, J., Kim, S. H., Kim, Y. S., \& Lee, Y. H. (2006). Clozapine, a neuroleptic agent, inhibits Akt by counteracting Ca2+/calmodulin in PTEN-negative 
U-87MG human glioblastoma cells. Cell Signal, 18(11), 1876-1886.

doi:10.1016/j.cellsig.2006.02.004

Smith-Garvin, J. E., Koretzky, G. A., \& Jordan, M. S. (2009). T Cell Activation. Annual review of immunology, 27, 591-619. doi:10.1146/annurev.immunol.021908.132706

Somer, E., Golan, D., Dishon, S., Cuzin-Disegni, L., Lavi, I., \& Miller, A. (2010). Patients with multiple sclerosis in a war zone: coping strategies associated with reduced risk for relapse. Mult Scler, 16(4), 463-471. doi:10.1177/1352458509358714

Sonobe, Y., Jin, S., Wang, J., Kawanokuchi, J., Takeuchi, H., Mizuno, T., \& Suzumura, A. (2007). Chronological Changes of CD $4<$ sup $>+</$ sup $>$ and $\operatorname{CD} 8<\sup >+</$ sup $>$ T Cell Subsets in the Experimental Autoimmune Encephalomyelitis, a Mouse Model of Multiple Sclerosis. The Tohoku Journal of Experimental Medicine, 213(4), 329-339. doi:10.1620/tjem.213.329

Sonobe, Y., Yawata, I., Kawanokuchi, J., Takeuchi, H., Mizuno, T., \& Suzumura, A. (2005). Production of IL-27 and other IL-12 family cytokines by microglia and their subpopulations. Brain Res, 1040(1-2), 202-207. doi:10.1016/j.brainres.2005.01.100

Sorenson, M., Janusek, L., \& Mathews, H. (2013). Psychological stress and cytokine production in multiple sclerosis: correlation with disease symptomatology. Biol Res Nurs, 15(2), 226-233. doi:10.1177/1099800411425703

Steiner, J., Bielau, H., Brisch, R., Danos, P., Ullrich, O., Mawrin, C., . . Bogerts, B. (2008). Immunological aspects in the neurobiology of suicide: elevated microglial density in schizophrenia and depression is associated with suicide. J Psychiatr Res, 42(2), 151157. doi:10.1016/j.jpsychires.2006.10.013

Steiner, J., Martins-de-Souza, D., Schiltz, K., Sarnyai, Z., Westphal, S., Isermann, B., . . . Keilhoff, G. (2014). Clozapine promotes glycolysis and myelin lipid synthesis in cultured oligodendrocytes. Front Cell Neurosci, 8, 384. doi:10.3389/fncel.2014.00384

Stephens, L. A., Gray, D., \& Anderton, S. M. (2005). CD4+CD25+ regulatory T cells limit the risk of autoimmune disease arising from T cell receptor crossreactivity. Proc Natl Acad Sci U S A, 102(48), 17418-17423. doi:10.1073/pnas.0507454102

Stephens, L. A., Malpass, K. H., \& Anderton, S. M. (2009). Curing CNS autoimmune disease with myelin-reactive Foxp3+ Treg. Eur J Immunol, 39(4), 1108-1117. doi:10.1002/eji.200839073

Stone, S., \& La Flamme, A. C. (2016). Type II Activation of Macrophages and Microglia by Immune Complexes Enhances Th17 Biasing in an IL-6-Independent Manner. PLoS One, 11(10), e0164454. doi:10.1371/journal.pone.0164454 
Strachan-Whaley, M., Rivest, S., \& Yong, V. W. (2014). Interactions between microglia and T cells in multiple sclerosis pathobiology. J Interferon Cytokine Res, 34(8), 615-622. doi:10.1089/jir.2014.0019

Sugino, H., Futamura, T., Mitsumoto, Y., Maeda, K., \& Marunaka, Y. (2009). Atypical antipsychotics suppress production of proinflammatory cytokines and up-regulate interleukin-10 in lipopolysaccharide-treated mice. Progress in NeuroPsychopharmacology and Biological Psychiatry, 33(2), 303-307. doi:http://dx.doi.org/10.1016/j.pnpbp.2008.12.006

Summers, C., Rankin, S. M., Condliffe, A. M., Singh, N., Peters, A. M., \& Chilvers, E. R. (2010). Neutrophil kinetics in health and disease. Trends in Immunology, 31(8), 318324. doi:10.1016/j.it.2010.05.006

Takeuchi, O., \& Akira, S. (2010). Pattern recognition receptors and inflammation. Cell, 140(6), 805-820. doi:10.1016/j.cell.2010.01.022

Tang, D., Kang, R., Coyne, C. B., Zeh, H. J., \& Lotze, M. T. (2012). PAMPs and DAMPs: Signal 0s that Spur Autophagy and Immunity. Immunol Rev, 249(1), 158-175. doi:10.1111/j.1600-065X.2012.01146.X

Teitelbaum, D., Meshorer, A., Hirshfeld, T., Arnon, R., \& Sela, M. (1971). Suppression of experimental allergic encephalomyelitis by a synthetic polypeptide. Eur J Immunol, 1(4), 242-248. doi:10.1002/eji.1830010406

Testa, J. R., \& Bellacosa, A. (2001). AKT plays a central role in tumorigenesis. Proc Natl Acad Sci U S A, 98(20), 10983-10985. doi:10.1073/pnas.211430998

Tierney, J. B., Kharkrang, M., \& La Flamme, A. C. (2008). Type II-activated macrophages suppress the development of experimental autoimmune encephalomyelitis. Immunol Cell Biol, 87(3), 235-240.

Toma, A., Haddouk, S., Briand, J.-P., Camoin, L., Gahery, H., Connan, F., . . Boitard, C. (2005). Recognition of a subregion of human proinsulin by class I-restricted $\mathrm{T}$ cells in type 1 diabetic patients. Proc Natl Acad Sci U S A, 102(30), 10581-10586. doi:10.1073/pnas.0504230102

Tompkins, S. M., Padilla, J., Dal Canto, M. C., Ting, J. P.-Y., Van Kaer, L., \& Miller, S. D. (2002). De Novo Central Nervous System Processing of Myelin Antigen Is Required for the Initiation of Experimental Autoimmune Encephalomyelitis. The Journal of Immunology, 168(8), 4173-4183. doi:10.4049/jimmunol.168.8.4173

Tone, Y., Furuuchi, K., Kojima, Y., Tykocinski, M. L., Greene, M. I., \& Tone, M. (2008). Smad3 and NFAT cooperate to induce Foxp3 expression through its enhancer. Nat 
Immunol, 9(2), 194-202.

doi:http://www.nature.com/ni/journal/v9/n2/suppinfo/ni1549 S1.html

Tremblay, M. E. (2011). The role of microglia at synapses in the healthy CNS: novel insights from recent imaging studies. Neuron Glia Biol, 7(1), 67-76.

doi:10.1017/s1740925x12000038

Tsang, B., \& Macdonell, R. (2011). Multiple sclerosis Diagnosis, management and prognosis. Australian Family Physician, 40, 948-955.

Turalba, A. V., Leite-Morris, K. A., \& Kaplan, G. B. (2004). Antipsychotics regulate cyclic AMP-dependent protein kinase and phosphorylated cyclic AMP response elementbinding protein in striatal and cortical brain regions in mice. Neurosci Lett, 357(1), 53-57. doi:10.1016/j.neulet.2003.11.059

Underhill, D. M., \& Goodridge, H. S. (2012). Information processing during phagocytosis. Nat Rev Immunol, 12(7), 492-502.

van Oosterwijk, M. F., Juwana, H., Arens, R., Tesselaar, K., van Oers, M. H., Eldering, E., \& van Lier, R. A. (2007). CD27-CD70 interactions sensitise naive CD4+ T cells for IL12-induced Th1 cell development. Int Immunol, 19(6), 713-718. doi:10.1093/intimm/dxm033

Vesterinen, H. M., Connick, P., Irvine, C. M., Sena, E. S., Egan, K. J., Carmichael, G. G., . . . Chandran, S. (2015). Drug repurposing: a systematic approach to evaluate candidate oral neuroprotective interventions for secondary progressive multiple sclerosis. PLoS One, 10(4), e0117705. doi:10.1371/journal.pone.0117705

Vieira, P. L., Heystek, H. C., Wormmeester, J., Wierenga, E. A., \& Kapsenberg, M. L. (2003). Glatiramer acetate (copolymer-1, copaxone) promotes Th2 cell development and increased IL-10 production through modulation of dendritic cells. J Immunol, 170(9), 4483-4488.

Vignali, D. A. A., Collison, L. W., \& Workman, C. J. (2008). How regulatory T cells work. Nat Rev Immunol, 8(7), 523-532.

Vitiello, A., Potter, T. A., \& Sherman, L. A. (1990). The role of beta 2-microglobulin in peptide binding by class I molecules. Science, 250(4986), 1423-1426.

Vogel, D. Y., Vereyken, E. J., Glim, J. E., Heijnen, P. D., Moeton, M., van der Valk, P., . . . Dijkstra, C. D. (2013). Macrophages in inflammatory multiple sclerosis lesions have an intermediate activation status. J Neuroinflammation, 10, 35. doi:10.1186/17422094-10-35 
Vogel, D. Y. S., Vereyken, E. J. F., Glim, J. E., Heijnen, P. D. A. M., Moeton, M., van der Valk, P., . . Dijkstra, C. D. (2013). Macrophages in inflammatory multiple sclerosis lesions have an intermediate activation status. J Neuroinflammation, 10, 35-35. doi:10.1186/1742-2094-10-35

Walker, L. S. K., \& von Herrath, M. (2016). CD4 T cell differentiation in type 1 diabetes. Clinical and Experimental Immunology, 183(1), 16-29. doi:10.1111/cei.12672

Wan, Y. Y. (2010). Regulatory T cells: immune suppression and beyond. Cellular and Molecular Immunology, 7(3), 204-210. doi:10.1038/cmi.2010.20

Weber, M. S., Prod'homme, T., Youssef, S., Dunn, S. E., Rundle, C. D., Lee, L., . . Zamvil, S. S. (2007). Type II monocytes modulate T cell-mediated central nervous system autoimmune disease. Nat Med, 13(8), 935-943. doi:10.1038/nm1620

Weber, M. S., Starck, M., Wagenpfeil, S., Meinl, E., Hohlfeld, R., \& Farina, C. (2004). Multiple sclerosis: glatiramer acetate inhibits monocyte reactivity in vitro and in vivo. Brain, 127(Pt 6), 1370-1378. doi:10.1093/brain/awh163

Weichhart, T., Costantino, G., Poglitsch, M., Rosner, M., Zeyda, M., Stuhlmeier, K. M., .. . Saemann, M. D. (2008). The TSC-mTOR signaling pathway regulates the innate inflammatory response. Immunity, 29(4), 565-577. doi:10.1016/j.immuni.2008.08.012

Weinmann, S., Read, J., \& Aderhold, V. (2009). Influence of antipsychotics on mortality in schizophrenia: systematic review. Schizophr Res, 113(1), 1-11. doi:10.1016/j.schres.2009.05.018

Wing, K., \& Sakaguchi, S. (2010). Regulatory T cells exert checks and balances on self tolerance and autoimmunity. Nat Immunol, 11(1), 7-13. doi:10.1038/ni.1818

Winkelmann, A., Loebermann, M., Reisinger, E. C., Hartung, H.-P., \& Zettl, U. K. (2016). Disease-modifying therapies and infectious risks in multiple sclerosis. Nat Rev Neurol, 12(4), 217-233. doi:10.1038/nrneurol.2016.21

Wujek, J. R., Bjartmar, C., Richer, E., Ransohoff, R. M., Yu, M., Tuohy, V. K., \& Trapp, B. D. (2002). Axon loss in the spinal cord determines permanent neurological disability in an animal model of multiple sclerosis. J Neuropathol Exp Neurol, 61(1), 23-32.

Wynn, T. A. (2015). Type 2 cytokines: mechanisms and therapeutic strategies. Nat Rev Immunol, 15(5), 271-282. doi:10.1038/nri3831

Wynn, T. A., Chawla, A., \& Pollard, J. W. (2013). Macrophage biology in development, homeostasis and disease. Nature, 496(7446), 445-455. doi:10.1038/nature12034

Xing, Z., Zganiacz, A., \& Santosuosso, M. (2000). Role of IL-12 in macrophage activation during intracellular infection: IL-12 and mycobacteria synergistically release TNF- 
alpha and nitric oxide from macrophages via IFN-gamma induction. J Leukoc Biol, 68(6), 897-902.

Xystrakis, E., Boswell, S. E., \& Hawrylowicz, C. M. (2006). T regulatory cells and the control of allergic disease. Expert Opin Biol Ther, 6(2), 121-133. doi:10.1517/14712598.6.2.121

Yamaguchi, T., Wing, J. B., \& Sakaguchi, S. (2011). Two modes of immune suppression by Foxp3(+) regulatory $\mathrm{T}$ cells under inflammatory or non-inflammatory conditions. Semin Immunol, 23(6), 424-430. doi:10.1016/j.smim.2011.10.002

Yamasaki, R., Lu, H., Butovsky, O., Ohno, N., Rietsch, A. M., Cialic, R., . . Ransohoff, R. M. (2014). Differential roles of microglia and monocytes in the inflamed central nervous system. The Journal of Experimental Medicine, 211(8), 1533-1549. doi:10.1084/jem.20132477

Yednock, T. A., Cannon, C., Fritz, L. C., Sanchez-Madrid, F., Steinman, L., \& Karin, N. (1992). Prevention of experimental autoimmune encephalomyelitis by antibodies against [alpha]4[beta]1 integrin. Nature, 356(6364), 63-66.

Yednock, T. A., Cannon, C., Fritz, L. C., Sanchez-Madrid, F., Steinman, L., \& Karin, N. (1992). Prevention of experimental autoimmune encephalomyelitis by antibodies against alpha 4 beta 1 integrin. Nature, 356(6364), 63-66. doi:10.1038/356063a0

Yuan, X. Q., Qiu, G., Liu, X. J., Liu, S., Wu, Y., Wang, X., \& Lu, T. (2012). Fluoxetine promotes remission in acute experimental autoimmune encephalomyelitis in rats. Neuroimmunomodulation, 19(4), 201-208. doi:10.1159/000334095

Zhang, F., Zhou, H., Wilson, B. C., Shi, J. S., Hong, J. S., \& Gao, H. M. (2012). Fluoxetine protects neurons against microglial activation-mediated neurotoxicity. Parkinsonism Relat Disord, 18 Suppl 1, S213-217. doi:10.1016/s1353-8020(11)70066-9

Zhang, X., Koldzic, D. N., Izikson, L., Reddy, J., Nazareno, R. F., Sakaguchi, S., . . Weiner, H. L. (2004). IL-10 is involved in the suppression of experimental autoimmune encephalomyelitis by CD25+CD4+ regulatory T cells. Int Immunol, 16(2), 249-256.

Zhang, X., Koldzic, D. N., Izikson, L., Reddy, J., Nazareno, R. F., Sakaguchi, S., . . Weiner, H. L. (2004). IL-10 is involved in the suppression of experimental autoimmune encephalomyelitis by CD25+CD4+ regulatory T cells. International Immunology, 16(2), 249-256. doi:10.1093/intimm/dxh029

Zhou, F., Ciric, B., Li, H., Yan, Y., Li, K., Cullimore, M., . . Rostami, A. (2012). IL-10 deficiency blocks the ability of LPS to regulate expression of tolerance-related 
molecules on dendritic cells. Eur J Immunol, 42(6), 1449-1458.

doi:10.1002/eji.201141733

Zhou, J., Cai, W., Jin, M., Xu, J., Wang, Y., Xiao, Y., . . Huang, R. (2015). 18betaglycyrrhetinic acid suppresses experimental autoimmune encephalomyelitis through inhibition of microglia activation and promotion of remyelination. Sci Rep, 5, 13713. doi:10.1038/srep13713

Zhu, J., Yamane, H., \& Paul, W. E. (2010). Differentiation of effector CD4 T cell populations (*). Annu Rev Immunol, 28, 445-489. doi:10.1146/annurev-immunol-030409-101212

Ziegler, S. F. (2006). FOXP3: of mice and men. Annu Rev Immunol, 24, 209-226. doi:10.1146/annurev.immunol.24.021605.090547 
Appendices 


\section{Appendix I}

Enhanced disease reduction using clozapine, an atypical antipsychotic agent, and glatiramer acetate combination therapy in experimental autoimmune encephalomyelitis
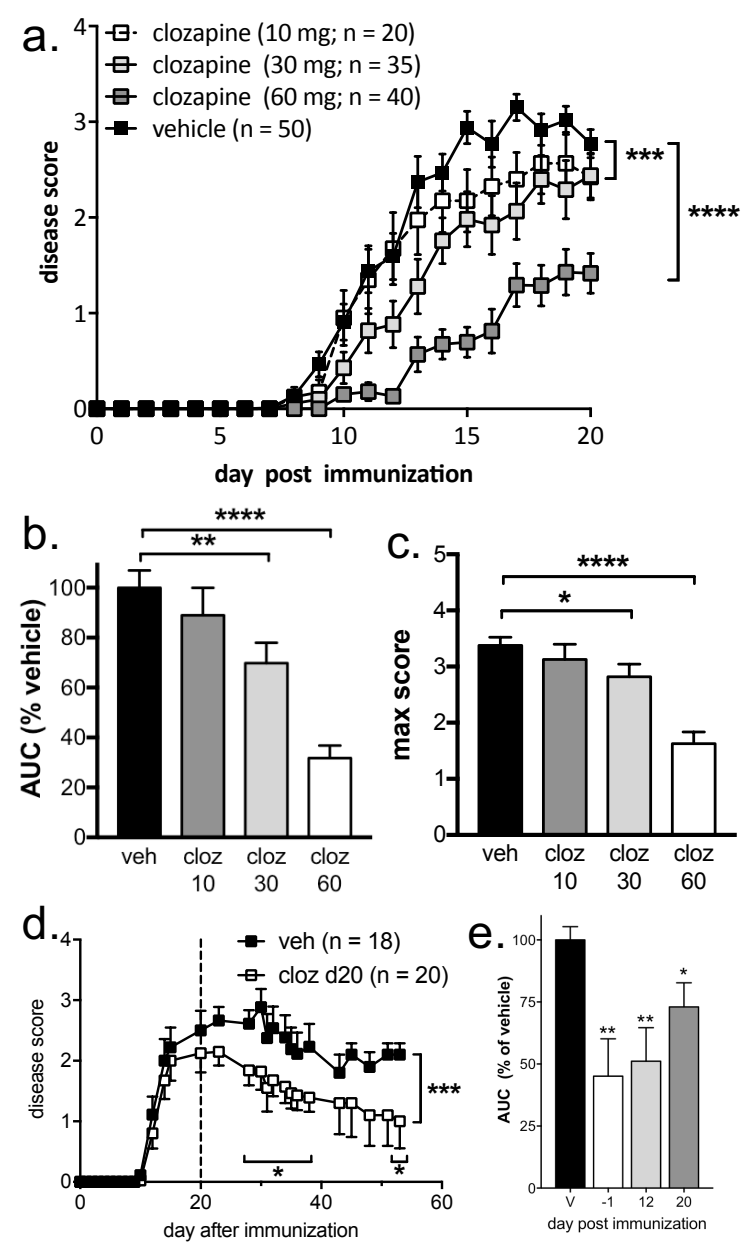

\section{Clozapine reduces EAE disease severity prophylactically and therapeutically}

a-e Clozapine reduces EAE disease severity prophylactically (a-c) and therapeutically (d \& e). (a) C57BL/6 female mice were treated with clozapine $(10,30$, and $60 \mathrm{mg} / \mathrm{kg} /$ day) or vehicle control in their drinking water commencing one day prior to immunization and were scored daily. Shown are the means and SEM of scores from individual mice $(\mathrm{n}=20-50$ mice as indicated) from 10 independent experiments. $* * * \mathrm{p}<0.001$ and $* * * * \mathrm{p}<0.0001$ compared to vehicle control by 2-way ANOVA. (b \& c) Clozapine treatment led to a dose-dependent reduction in overall disease burden as assessed by area under the curve (AUC) compared to the vehicle control and reduced peak disease score. Shown are the means and SEM of values from individual mice $(\mathrm{n}=20-50 /$ group as indicated in a). $* p<0.05, * * p<0.01$ and $* * * * p<0.0001$ compared to vehicle control by 1-way ANOVA with HolmSidak's multiple comparison test (b) or Kruskal-Wallis with Dunn's multiple comparison test (c). (d \& e) Clozapine reduces EAE severity when administered before or 12 or 20 days after immunization. Shown are the means and SEM of values from individual mice $(n=18-20$ /group, veh and d20; $n=10$ /group d-1 and d12) from 3 independent experiments. ${ }^{*} p<0.05, * * p<0.01$, and $* * * p<0.001$ by 2 -way ANOVA with Fisher's LSD test (d) and compared to vehicle control by 1-way ANOVA with Holm-Sidak's multiple comparison test (e). Figure taken from accepted manuscript (L. Green et al., 2017). 


\section{Appendix II}

\section{Flow cytometry gating strategies}

A
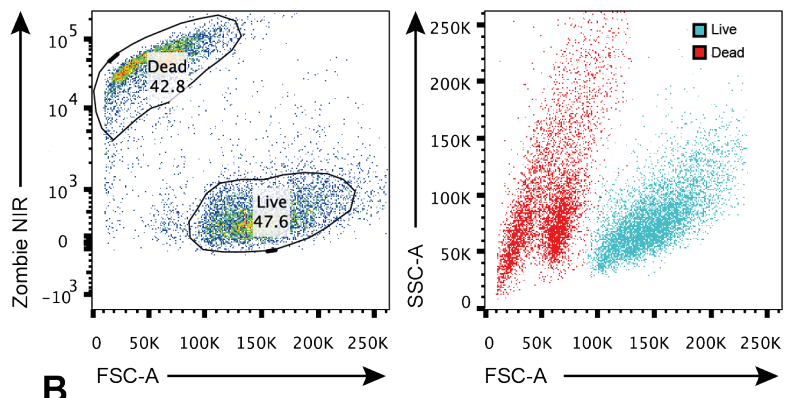

B
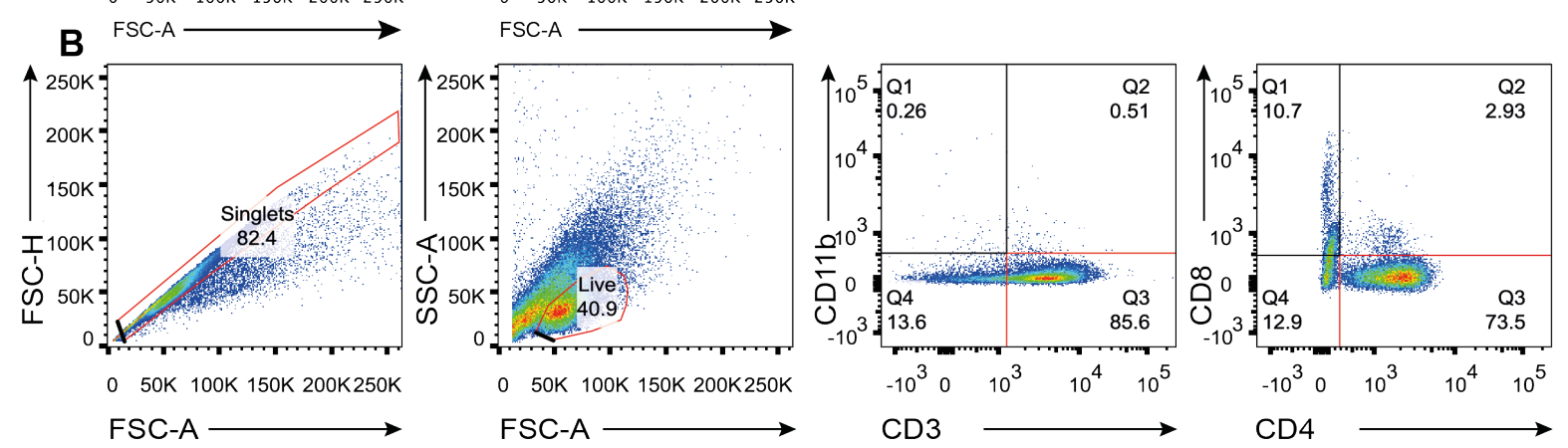

\section{Gating strategy for identifying CD4 $T$ cells in cell culture}

A Gating strategy for identifying dead and live cells from stimulated CD4 T cell cultures assessed by exclusion of Zombie NIR (Left). Overlay of Live and Dead cells by FSC-A vs SSC-A to show that dead cells (shown in red) are FSC-A ${ }^{\text {low }}$ and have a larger spread in SSC-A than live cells (shown in blue). B Firstly, singlets were gated by FSC-A v FSH-H and then live cells were identified by FSC-A and SSC-A based on size and granularity according to A. Next, lymphocytes were identified by CD11b- and CD3+ followed by CD8 v CD4 discrimination. Gating is shown sequentially from left to right and selected gates are outlined in red. 

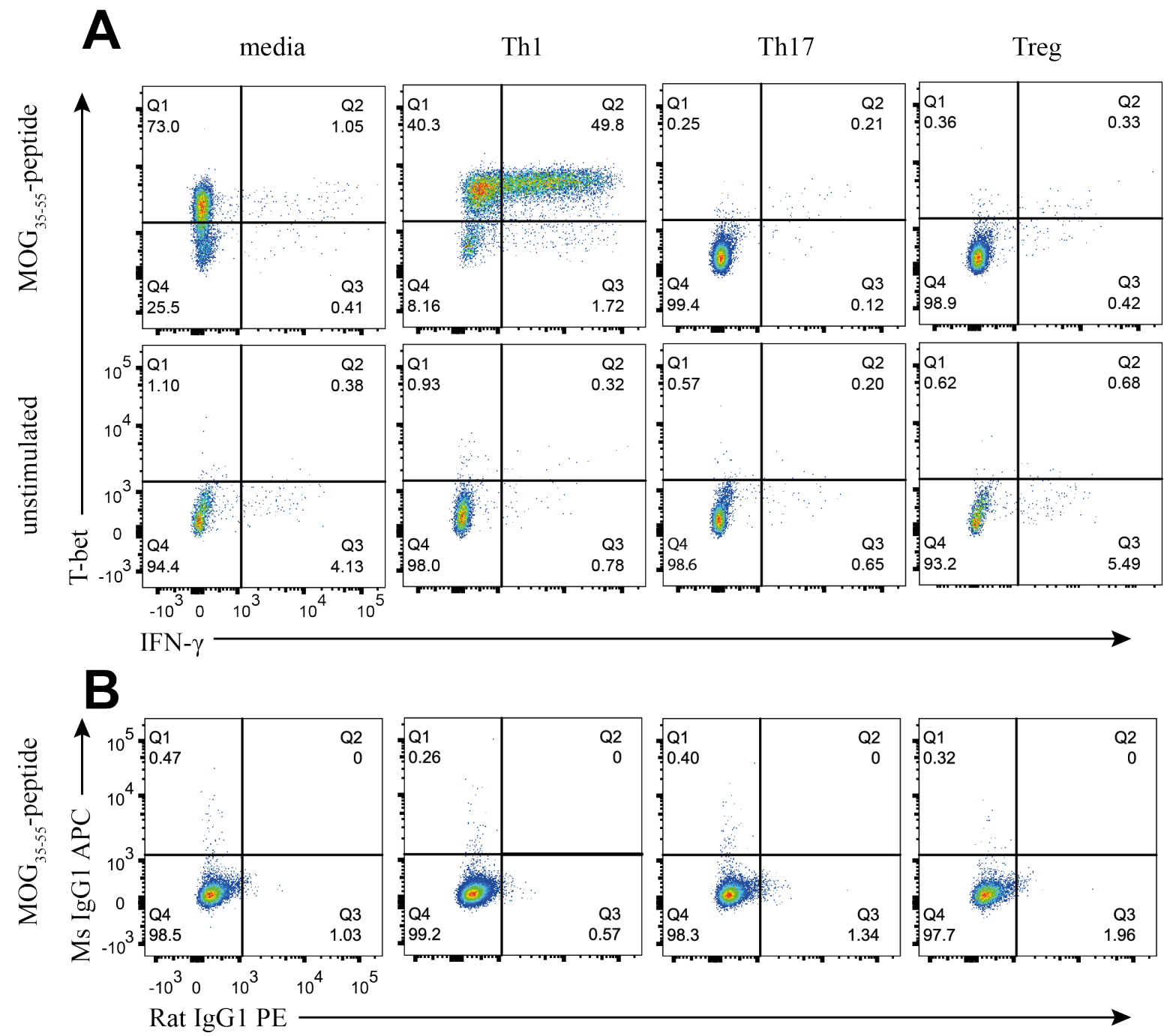

\section{Gating Strategy for identifying Th1 cells after $\mathbf{T}$ cell differentiation}

Firstly, CD4 T cells were identified and gated as described above. Gates for T-bet and IFN- $\gamma$ were set using stimulated samples as a positive control and unstimulated controls as a negative control as shown. Shown are representative dot plots identifying Th1 cells in media, Th1 (20 ng/mL rIL-12p70, $10 \mu \mathrm{g} / \mathrm{mL}$ anti-IL-4), Th17 (5 ng/mL rTGF- $\beta 1,20 \mathrm{ng} / \mathrm{mL}$ rIL-6, $10 \mu \mathrm{g} / \mathrm{mL}$ anti-IFN- $\gamma$ ) and Treg $(5 \mathrm{ng} / \mathrm{mL}$ rTGF- $\beta 1,10 \mu \mathrm{g} / \mathrm{mL}$ anti-IL-6, $10 \mu \mathrm{g} / \mathrm{mL}$ anti-IFN- $\gamma$ ) after 72 hours. 

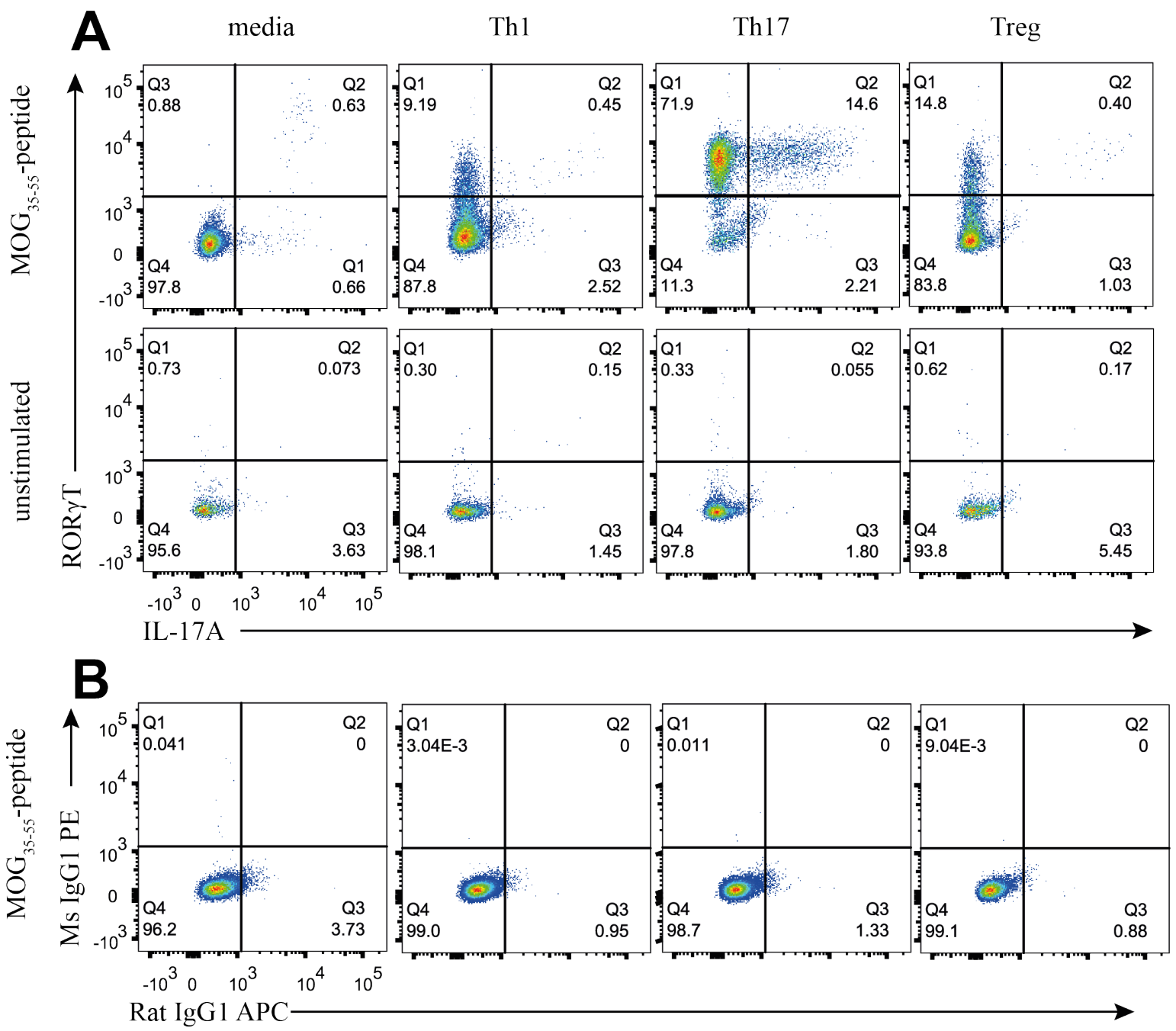

\section{Flow cytometry gating strategy for identifying Th17 cells}

Firstly, CD4 T cells were identified as described above. Gates for ROR $\gamma$ T and IL-17A were set using stimulated samples as a positive control and unstimulated controls as a negative control as shown. Shown are representative dot plots identifying Th17 cells in media, Th1 (20 ng/mL rIL-12p70, $10 \mu \mathrm{g} / \mathrm{mL}$ anti-IL-4), Th17 (5 ng/mL rTGF- $\beta 1,20 \mathrm{ng} / \mathrm{mL}$ rIL-6, $10 \mu \mathrm{g} / \mathrm{mL}$ anti-IFN- $\gamma$ ) and Treg $(5 \mathrm{ng} / \mathrm{mL}$ rTGF- $\beta 1,10 \mu \mathrm{g} / \mathrm{mL}$ anti-IL-6, $10 \mu \mathrm{g} / \mathrm{mL}$ anti-IFN- $\gamma$ ) after 72 hours. 


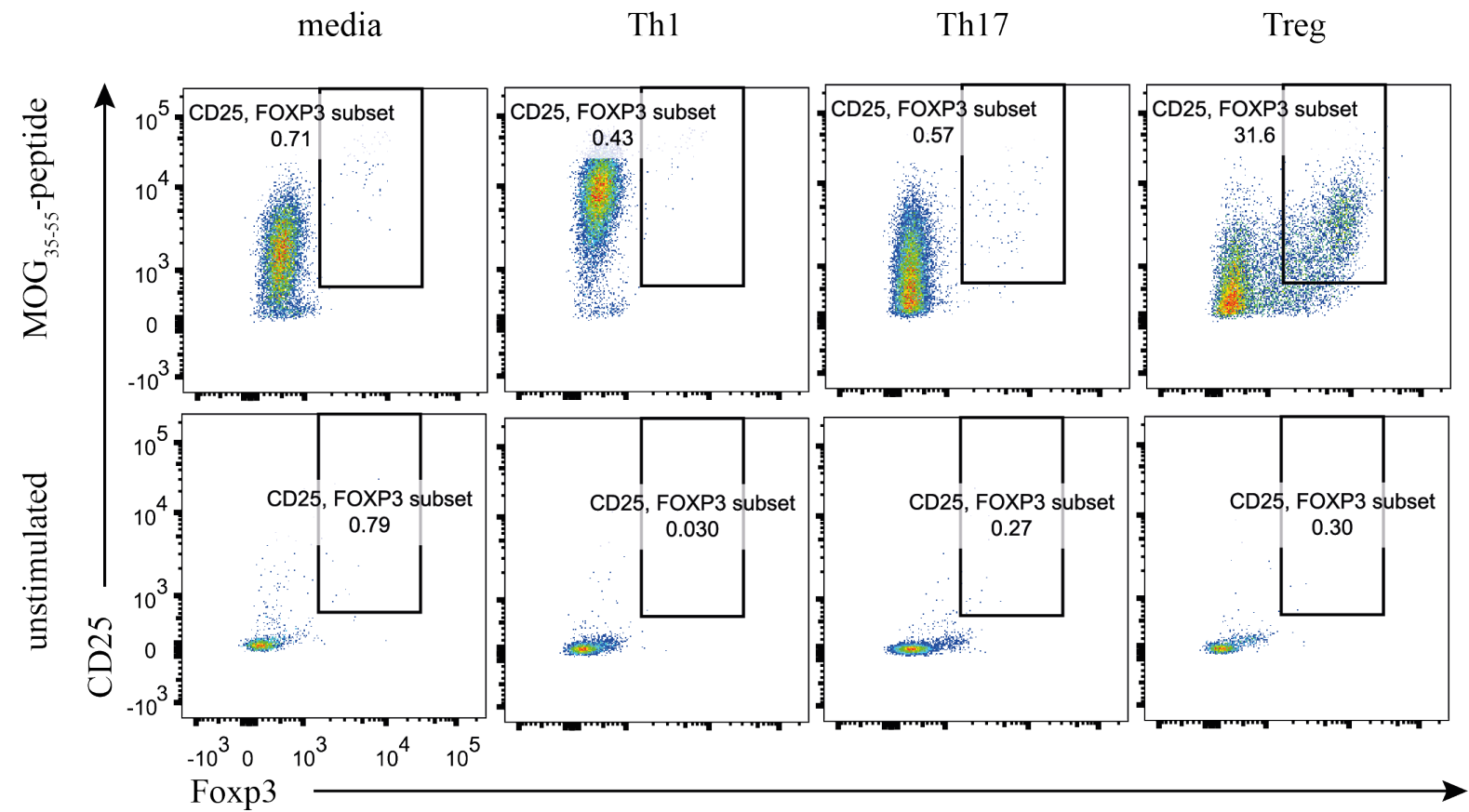

\section{Flow cytometry gating strategy for identifying Treg cells}

Firstly, CD4 T cells were identified as described above. Gates for Foxp3 were set using stimulated samples as a positive control and unstimulated controls as a negative control as shown. Shown are representative dot plots identifying Treg cells in media, Th1 (20 ng/mL rIL-12p70, $10 \mu \mathrm{g} / \mathrm{mL}$ anti-IL-4), Th17 (5 ng/mL rTGF- $\beta 1,20$ $\mathrm{ng} / \mathrm{mL}$ rIL-6, $10 \mu \mathrm{g} / \mathrm{mL}$ anti-IFN- $\gamma$ ) and Treg $(5 \mathrm{ng} / \mathrm{mL}$ rTGF- $\beta 1,10 \mu \mathrm{g} / \mathrm{mL}$ anti-IL-6, $10 \mu \mathrm{g} / \mathrm{mL}$ anti-IFN- $\gamma)$ after 72 hours. Isotype control for mouse IgG1 anti-Foxp3 antibody as per gating strategy for identifying Th1 cells shows no non-specific staining. 

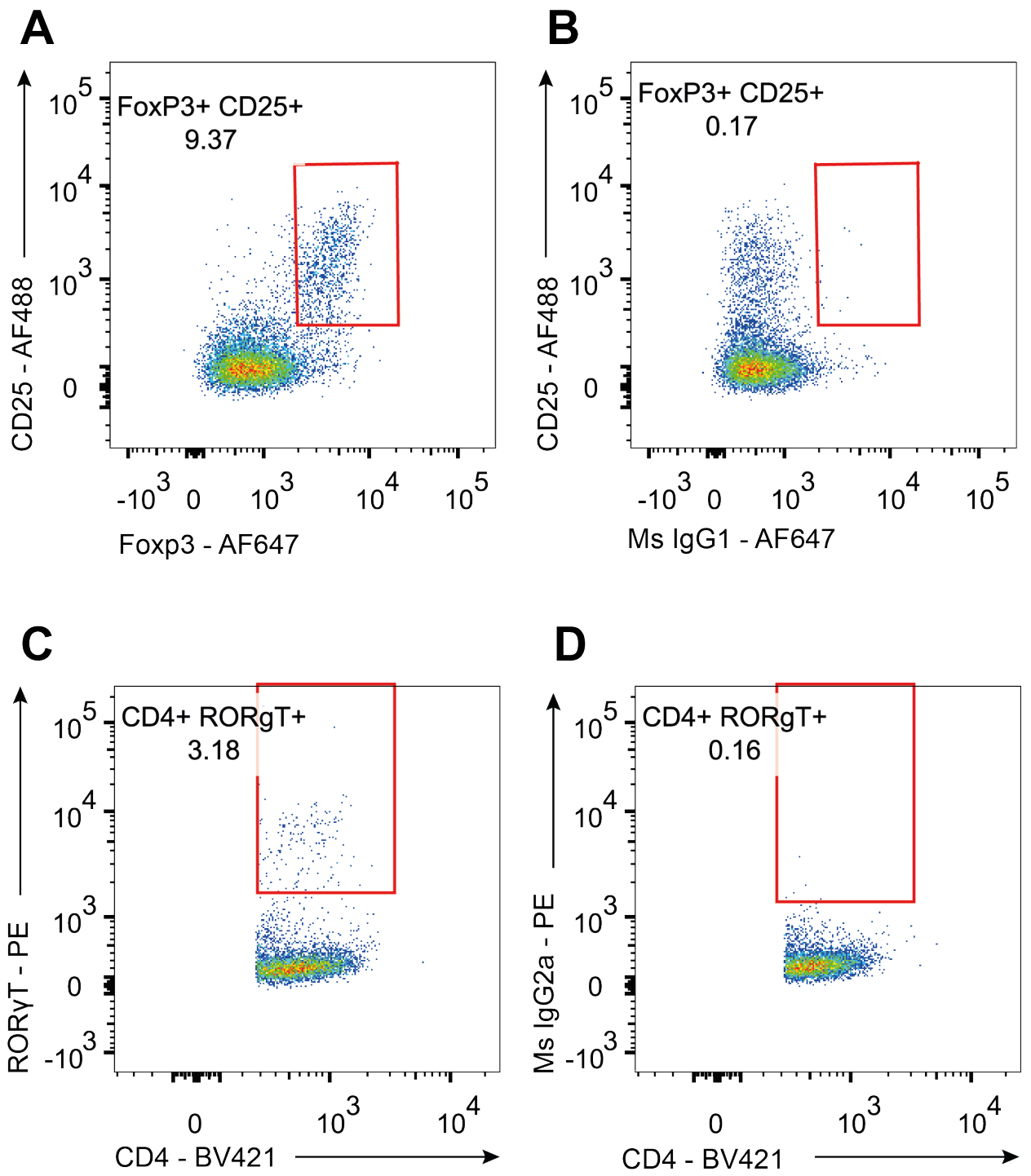

\section{D}

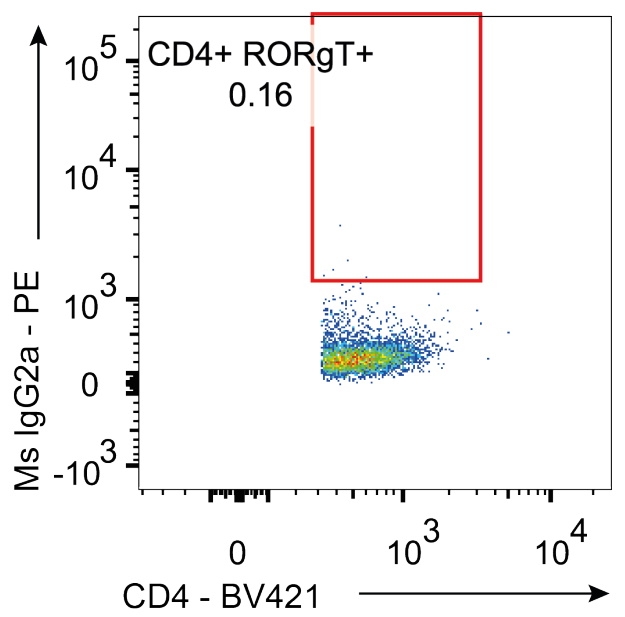

\section{Rationale for identifying Foxp3 ${ }^{+}$Treg and ROR $\gamma \mathrm{T}^{+}$Th17 cells in splenocytes ex vivo.}

$\mathrm{CD}^{+} \mathrm{T}$ cells were identified and gated as previously described. A-B Gating rationale for identifying Treg in the spleen. A Representative dot plot of CD25 v Foxp3 in the spleen of mice. B Representative dot plot of CD25 v Mouse IgG1 - AF647 isotype control (fluorescence minus one) from the same sample showing specific staining in A. C-D Gating rationale for identifying Th17 cells in the spleen. C Representative dot plot of ROR $\gamma$ T v CD4 in the spleen of mice. D Representative dot plot of Mouse IgG1 - PE isotype control (fluorescence minus one) from the same sample showing specific staining in $\mathbf{C}$. 

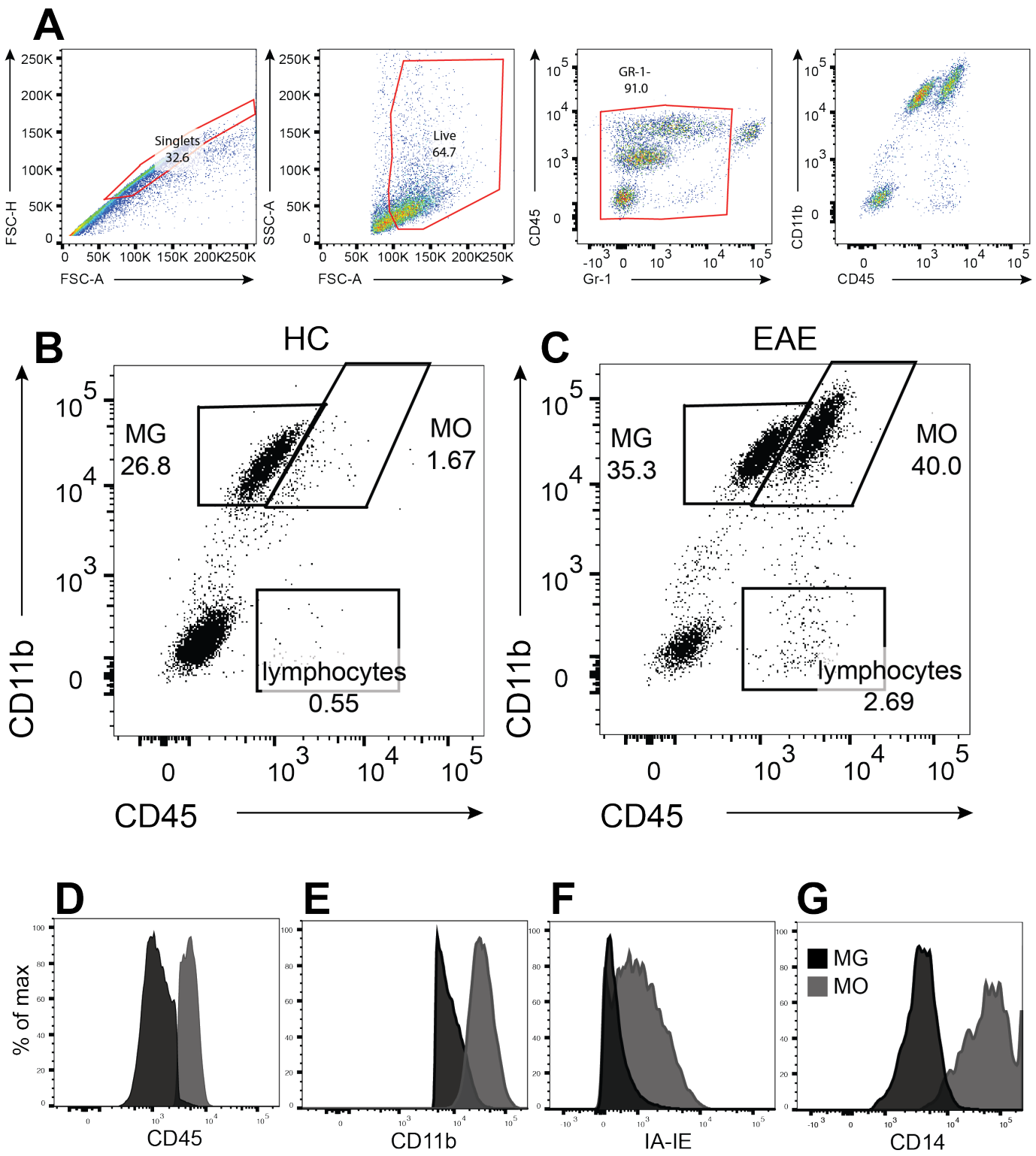

\section{Rationale for distinguishing infiltrating monocytes from CNS-resident microglia}

A-C Gating strategy to identify infiltrating monocytes and CNS-resident microglia. A Gating strategy for identifying monocytes and microglia (left to right). Single cells were gated by FSC-A v FSC-H (outlined in red). Live cells of interest (live) were identified by size and granularity using FSC-A v SSC-A to exclude dead cells and debris (outlined in red). Granulocytes were excluded from analysis by high Gr-1 expression using Gr1 v CD45 (outlined in red). B-C Representative dot plots from $\mathbf{B}$ healthy control (HC) or $\mathbf{C}$ EAE mice at 15 d.p.i after gating as in A. These dot plots show that infiltrating monocytes (MO) are present in high proportions only in mice with EAE (C) while CNS-resident microglia (MG) are present in both HC and EAE mice. D-G Representative histograms of expression of D CD45, E CD11b, F MHC Class II (IA-IE) and G CD14 from microglia and monocytes in a mouse with EAE showing that MG express lower levels of CD45, CD11b, MHC Class II and CD14 than infiltrating monocytes consistent with previously published studies (Butovsky et al., 2014; Hickman et al., 2013; Melief et al., 2012; Sedgwick et al., 1991). This demonstrates that the gating strategy used reasonably distinguishes microglia from infiltrating monocytes. 


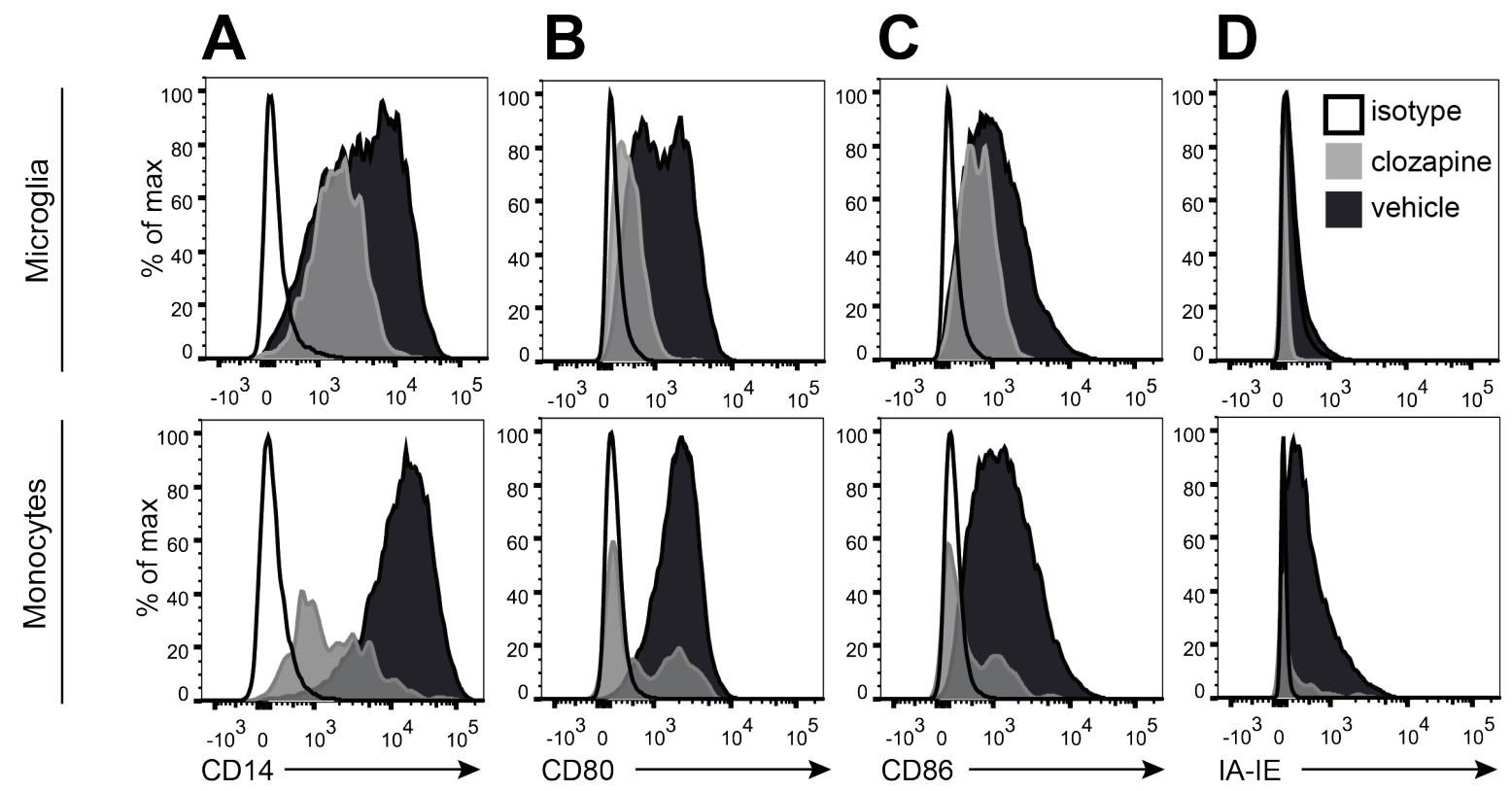

Histograms of CD14, CD80. CD86 and IA-IE expression in microglia and monocytes

A-D Representative histograms of A CD14, B CD80, C CD86 and D IA-IE expression in microglia and monocytes from vehicle and clozapine treated mice with EAE compared to isotype controls to show specific staining of antibody. 
A

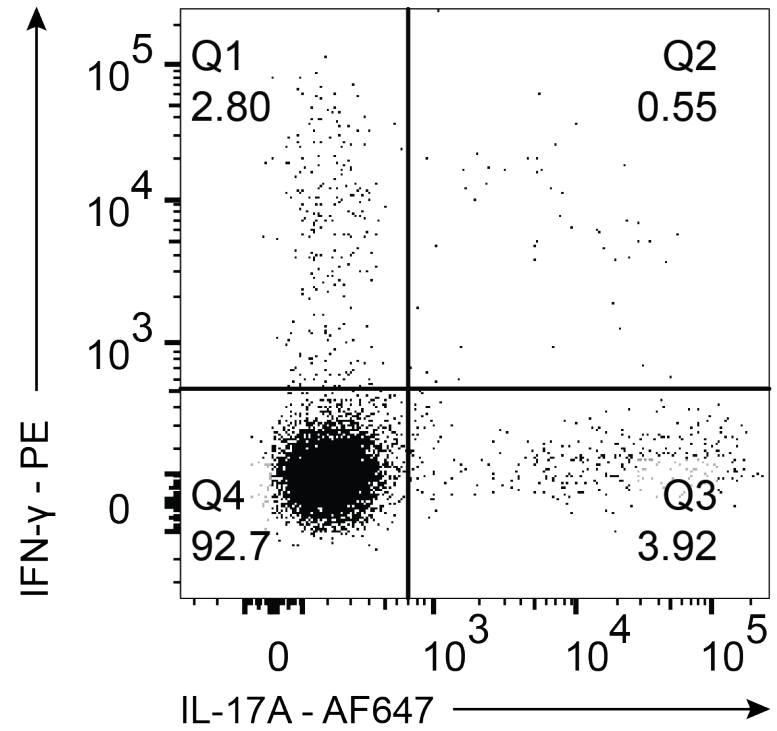

B

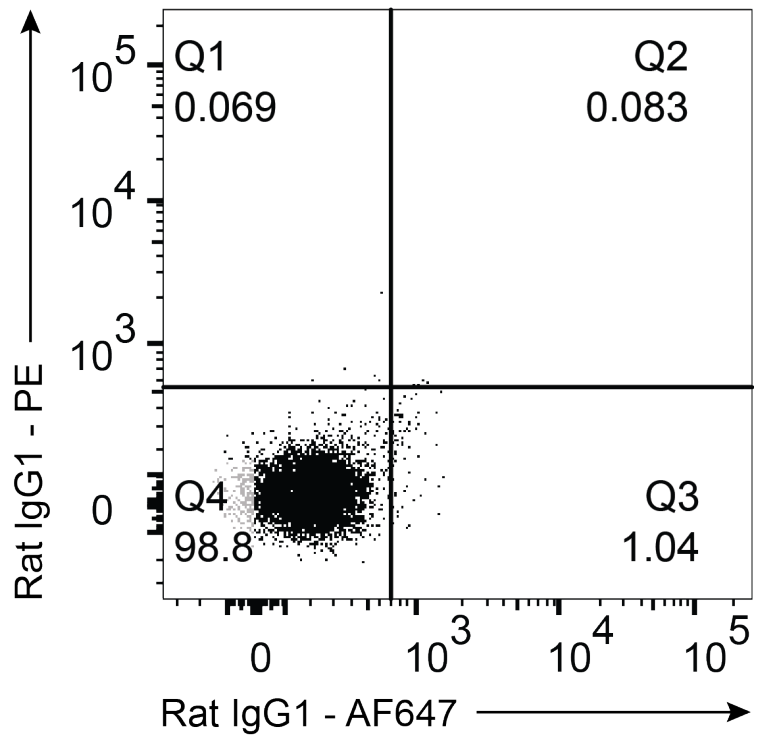

\section{Rationale for quadrant gating of IFN- $\gamma+$ and IL-17A + CD4 T cells}

$\mathrm{CD}^{+} \mathrm{T}$ cells were first identified by the gating strategy previously described. A-B Rationale for gating quadrants to identify IFN- $\gamma^{+}$and IL-17A ${ }^{+}$CD4 T cells in the spleen. A Representative dot plot of an EAE mouse using IFN- $\gamma$ v IL-17A. B Representative dot plot of an EAE mouse using Rat IgG1 - PE isotype control v Rat IgG1 AF647 isotype control. Quadrant gates were set using A as a positive control and B as a negative control to identify IFN- $\gamma^{+}$and IL-17A ${ }^{+} \mathrm{T}$ cells. 


\section{Appendix III}

Buffers and Solutions

10x Phosphate buffered saline

$\mathrm{NaCl} 170$ g

$\mathrm{Na}_{2} \mathrm{HPO}_{4} .12 \mathrm{H}_{2} \mathrm{O} 62.32 \mathrm{~g}$

$\mathrm{NaH}_{2} \mathrm{PO}_{4} .2 \mathrm{H}_{2} \mathrm{O} 4.04 \mathrm{~g}$

2 litres $\mathrm{ddH}_{2} \mathrm{O}$

Cryopreservation medium

Foetal calf serum $\mathbf{9 0 \%}$

DMSO 10\%

Lysis buffer

HEPES 5 mM

Tween-20 0.5\%

in PBS

Percoll@ Diluent $(180 \mathrm{~mL})$

10x PBS 45 ml

$0.6 \mathrm{M} \mathrm{HCl} 3 \mathrm{ml}$

$\mathrm{ddH}_{2} \mathrm{O} \mathbf{1 3 2} \mathbf{m l}$

Sterile filter through $0.22 \mu \mathrm{M}$ syringe filter 
$37 \%$ Percoll

Percoll@ 33.4\%

Percoll@ diluent 19.6\%

PBS 47\%

FACS buffer (v/v)

Foetal calf serum $\mathbf{2 \%}$

Sodium azide (1 M) $\mathbf{0 . 1 \%}$

PBS 97.9\%

Pertussis toxin buffer $(50 \mathrm{~mL})$

$1 \mathrm{M}$ Tris $\mathbf{0 . 7 5} \mathbf{~ m L}$

$\mathrm{NaCl} 0.025$ mL

Triton X-100 0.0085 mL

ddH2O 49.2165 mL

Sterile filter using $0.22 \mu \mathrm{m}$ syringe filter

Complete T cell media (CTCM; $582 \mathrm{~mL}$ )

Dulbecco’s Modified Eagle Medium 500 mL

Foetal calf serum $\mathbf{5} \mathbf{~ m L}$

$\beta$-Mercaptoethanol (55 mM) $\mathbf{0 . 5 8 2 5} \mathbf{~ m L}$

Non-essential amino acids (10 nM) 5.825 mL

L-glutamate (200 mM) 5.825 mL 
HEPES buffer (1 M) $\mathbf{5 . 8 2 5} \mathbf{~ m L}$

Penicillin/Streptomycin (100 U/ml/10 mg/ml) $\mathbf{5 . 8 2 5}$ mL

Wash buffer (520.83 mL)

Dulbecco’s Modified Eagle Medium 500 mL

HEPES buffer (1M) $\mathbf{1 5 . 6 3 ~} \mathbf{~ m L}$

Penicillin/Streptomycin $(100 \mathrm{U} / \mathrm{ml} / 10 \mathrm{mg} / \mathrm{ml}) \mathbf{5 . 2} \mathbf{~ m L}$

MTT Solution

MTT (in PBS) $5 \mathbf{~ m g / m L ~}$

Sterile filter through $0.22 \mu \mathrm{M}$ syringe filter

MTT Solubiliser

SDS $10 \%$

DMF 45\%

$\mathrm{dH}_{2} \mathrm{O} 45 \%$

$\mathrm{pH}$ to 4.5 with acetic acid

ELISA capture buffer

0.1M Na $2 \mathrm{HPO}_{4}$

$\mathrm{pH}$ to 9.0

ELISA stop solution 
$0.18 \mathrm{M} \mathrm{H}_{2} \mathrm{SO}_{4}$

Isolation buffer $(50 \mathrm{~mL})$

Foetal calf serum $\mathbf{1} \mathbf{~ m L}$

$0.5 \mathrm{M}$ EDTA $200 \mu \mathbf{L}$

PBS 48.8 mL

4\% Paraformaldehyde $(w / v)$

Paraformaldehyde $\mathbf{4}$ g

PBS up to $100 \mathrm{~mL}$

$\mathrm{pH}$ adjusted to 7.4

$30 \%$ Sucrose

Sucrose (w/v) 30\%

PBS 70\%

\section{Appendix IV}

\section{Antibodies and Dyes}

\begin{tabular}{llllll} 
Antibody & Clone & Fluorophore & Isotype & Manufacturer & Dilution \\
\hline CD3 & $17 \mathrm{~A} 2$ & APC/Cy7 & Rat IgG2b, $\kappa$ & Biolegend, CA & $1: 200$ \\
CD4 & GK1.5 & BV421 & Rat IgG2b, $\kappa$ & Biolegend, CA & $1: 400$
\end{tabular}




\begin{tabular}{|c|c|c|c|c|c|}
\hline CD4 & GK1.5 & PE & Rat $\operatorname{IgG} 2 \mathrm{a}, \mathrm{\kappa}$ & BD Pharmingen, NJ & $1: 800$ \\
\hline CD8 & $53-6.7$ & PerCP-Сy5.5 & Rat $\operatorname{IgG} 2 \mathrm{a}, \mathrm{\kappa}$ & Biolegend, CA & $1: 800$ \\
\hline CD11b & $\mathrm{M} 1 / 70$ & AF488 & Rat $\operatorname{IgG} 2 b, \kappa$ & Biolegend, CA & $1: 1000$ \\
\hline CD11b & $\mathrm{M} 1 / 70$ & $\mathrm{PE} / \mathrm{Cy} 7$ & Rat $\operatorname{IgG} 2 b, \kappa$ & Biolegend, CA & $1: 1000$ \\
\hline CD14 & RTK2758 & $\mathrm{PE} / \mathrm{Cy} 7$ & Rat IgG2a, $\kappa$ & Biolegend, CA & $1: 1000$ \\
\hline CD25 & PC61 & AF488 & Rat IgG1, $\lambda$ & Biolegend, CA & $1: 400$ \\
\hline & & & Armenian Hamster & & \\
\hline CD80 & $16-10 \mathrm{~A} 1$ & $\mathrm{PE}$ & IgG & Biolegend, CA & $1: 100$ \\
\hline CD86 & GL-1 & PE & Rat IgG2a, $\kappa$ & Biolegend, CA & $1: 100$ \\
\hline CD40 & $3 / 23$ & PE & Rat IgG2a, $\kappa$ & BD Pharmingen, NJ & $1: 100$ \\
\hline CD45 & $30-\mathrm{F} 11$ & BV510 & Rat $\operatorname{IgG} 2 b, \kappa$ & BD Pharmingen, NJ & $1: 400$ \\
\hline CD45.1 & A20 & PE & Mouse IgG2a, к & BD Pharmingen, NJ & $1: 400$ \\
\hline CD45.2 & 104 & APC & Mouse IgG2a, к & BD Pharmingen, NJ & $1: 400$ \\
\hline & & & & Thermo & \\
\hline $\mathrm{F} 4 / 80$ & BM8 & PerCP-Cy5.5 & Rat $\operatorname{IgG} 2 \mathrm{a}, \mathrm{\kappa}$ & Scientific, MA & $1: 400$ \\
\hline Gr-1 & RB6-8C5 & APC-Cy7 & Rat $\operatorname{IgG} 2 b, \kappa$ & BD Pharmingen, NJ & $1: 400$ \\
\hline IA/IE & M5/114.15.2 & BV421 & Rat $\operatorname{IgG} 2 b, \kappa$ & Biolegend, CA & $1: 400$ \\
\hline Biotin V $\beta 11$ & RR3-15 & - & Rat IgG2b, $\kappa$ & BD Pharmingen, NJ & $1: 400$ \\
\hline $\mathrm{V} \alpha 3.2$ & RR3-16 & FITC & Rat IgG2b, $\kappa$ & BD Pharmingen, NJ & \\
\hline rabbit anti & & & & & \\
\hline D1R & - & - & Rabbit IgG & Merck Millipore, MA & $1: 100$ \\
\hline rabbit anti & & & & & \\
\hline $\mathrm{D} 2 \mathrm{R}$ & - & - & Rabbit IgG & Merck Millipore, MA & $1: 100$ \\
\hline rabbit anti $\beta 2$ & & & & Abcam, Cambridge, & \\
\hline AR & - & - & Rabbit IgG & UK & $1: 100$ \\
\hline $\begin{array}{l}\text { Goat anti- } \\
\text { rabbit IgG }\end{array}$ & - & FITC & Goat IgG & BD Pharmingen, NJ & $1: 100$ \\
\hline
\end{tabular}




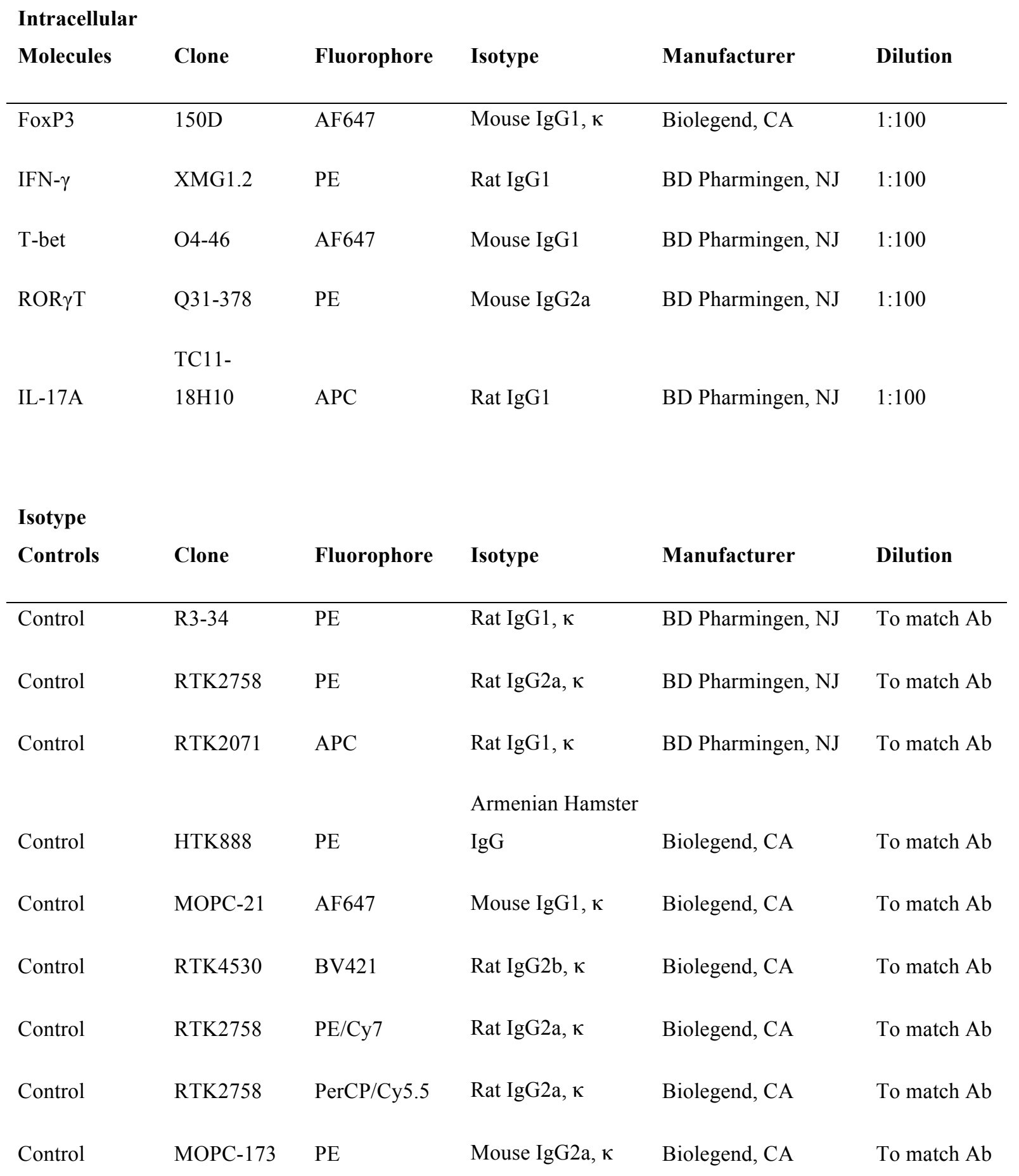




\section{Appendix V}

\section{ELISA Reagents and concentrations}

IFN- $\gamma$ ELISA Reagents

\begin{tabular}{l|l|l|l} 
Reagent & Concentration & Diluent & Incubation \\
\hline $\begin{array}{l}\text { Purified rat anti mouse } \\
\text { IFN- } \gamma \text { (BD Biosciences, } \\
\text { CA) }\end{array}$ & $1 \mu \mathrm{g} / \mathrm{mL}$ & $0.1 \mathrm{M} \mathrm{Na}_{2} \mathrm{HPO}_{4}, \mathrm{pH} 9.0$ & ${\text { overnight } 4^{\circ} \mathrm{c}}^{\circ}$ \\
\hline Block (FCS) & $10 \%$ & PBS pH 7.4 & \\
\hline Top standard (rIFN- $\gamma$ ) & $4 \mathrm{ng} / \mathrm{mL}$ & PBS $+5 \% \mathrm{FCS}$ & 2 hours RT \\
\hline Biotinylated rat anti IFN- & $125 \mathrm{ng} / \mathrm{mL}$ & PBS $+5 \% \mathrm{FCS}$ & 1 hours RT \\
$\gamma$ & & & \\
(BD Biosciences, CA) & & & \\
\hline SA-HRP & $1: 2000$ & & \\
(BD Biosciences, CA) & & &
\end{tabular}

IL-12 ELISA Reagents

\begin{tabular}{|c|c|c|c|}
\hline Reagent & Concentration & Diluent & Incubation \\
\hline $\begin{array}{l}\text { Purified rat anti mouse } \\
\text { IL-12 p40/70 } \\
\text { (BD Biosciences, CA) }\end{array}$ & $1 \mu \mathrm{g} / \mathrm{mL}$ & $0.1 \mathrm{M} \mathrm{Na}_{2} \mathrm{HPO}_{4}, \mathrm{pH} 9.0$ & overnight $4^{\circ} \mathrm{c}$ \\
\hline Block (FCS) & $10 \%$ & PBS pH 7.4 & 2 hours RT \\
\hline Top standard (rIL-12p40) & $4 \mathrm{ng} / \mathrm{mL}$ & $\mathrm{PBS}+5 \% \mathrm{FCS}$ & 2 hours RT \\
\hline $\begin{array}{l}\text { Biotin rat anti IL-12 } \\
\text { (BD Biosciences, CA) }\end{array}$ & $1 \mu \mathrm{g} / \mathrm{mL}$ & $\mathrm{PBS}+5 \% \mathrm{FCS}$ & 1 hour RT \\
\hline $\begin{array}{l}\text { SA-HRP } \\
\text { (BD Biosciences, CA) }\end{array}$ & $1: 2000$ & $\mathrm{PBS}+5 \% \mathrm{FCS}$ & 1 hour RT \\
\hline
\end{tabular}


IL-10 ELISA Reagents

\begin{tabular}{l|l|l|l} 
Reagent & Concentration & Diluent & Incubation \\
\hline $\begin{array}{l}\text { Purified rat anti mouse } \\
\text { IL-10 }\end{array}$ & $5 \mu \mathrm{g} / \mathrm{mL}$ & $0.1 \mathrm{M} \mathrm{Na}_{2} \mathrm{HPO}_{4}, \mathrm{pH} 6.0$ & overnight $4^{\circ} \mathrm{c}$ \\
(BD Biosciences, CA) & & & \\
\hline Block (FCS) & $10 \%$ & PBS pH 7.4 & \\
\hline $\begin{array}{l}\text { Top standard (rIL-10) } \\
\text { anti }\end{array}$ & $0.25 \mu \mathrm{ng} / \mathrm{mL}$ & PBS $+5 \% \mathrm{FCS}$ & 2 hours RT \\
\hline $\begin{array}{l}\text { Biotinylated } \mathrm{RT} \\
\text { mouse IL-10 }\end{array}$ & & PBS $+5 \% \mathrm{FCS}$ & 1 hour RT \\
(BD Biosciences, CA) & & & \\
\hline SA-HRP & & & \\
(BD Biosciences, CA) & & & \\
\hline
\end{tabular}

IL-17A ELISA Reagents

\begin{tabular}{l|l|l|l} 
Reagent & Concentration & Diluent & Incubation \\
\hline $\begin{array}{l}\text { Purified rat anti mouse } \\
\text { IL-17A }\end{array}$ & $1 \mu \mathrm{g} / \mathrm{mL}$ & $0.1 \mathrm{M} \mathrm{Na}_{2} \mathrm{HPO}_{4}, \mathrm{pH} 9.0$ & overnight $4^{\circ} \mathrm{c}$ \\
\hline Block (FCS) & $10 \%$ & PBS pH 7.4 & \\
& & & 2 hours RT
\end{tabular}




\begin{tabular}{l|l|l|l} 
Top standard (rIL-17A & $2 \mathrm{ng} / \mathrm{mL}$ & PBS $+5 \%$ FCS & 2 hours RT \\
\hline $\begin{array}{l}\text { Biotinylated rat anti } \\
\text { mouse IL-17A }\end{array}$ & $1 \mu \mathrm{g} / \mathrm{mL}$ & PBS + 5\% FCS & 1 hour RT \\
$\begin{array}{l}\text { (BD Biosciences, CA) } \\
\text { SA-HRP }\end{array}$ & $1: 1000$ & PBS + 5\% FCS & 1 hour RT \\
(BD Biosciences, CA) & & &
\end{tabular}

